NIST NCSTAR 1-4A

Federal Building and Fire Safety Investigation of the World Trade Center Disaster

\title{
Post-Construction Fires Prior to September 11, 2001
}

Erica D. Kuligowski

David D. Evans

Richard D. Peacock

NST National Institute of Standards and Technology • Technology Administration • U.S. Department of Commerce 


\section{Federal Building and Fire Safety Investigation of the World Trade Center Disaster}

\section{Post-Construction Fires Prior to September 11, 2001}

Erica D. Kuligowski

Building and Fire Research Laboratory

National Institute of Standards and Technology

David D. Evans

Society of Fire Protection Engineers

Richard D. Peacock

Building and Fire Research Laboratory

National Institute of Standards and Technology

September 2005

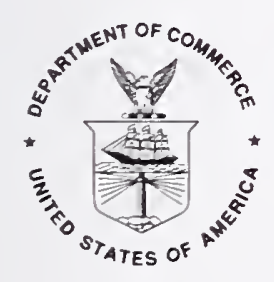

U.S. Department of Commerce

Carlos M. Gutierrez, Secretary

Technology Administration

Michelle O'Neill, Acting Under Secretary for Technology 


\section{Disclaimer No. 1}

Certain commercial entities, equipment, products, or materials are identified in this document in order to describe a procedure or concept adequately or to trace the history of the procedures and practices used. Such identification is not intended to imply recommendation, endorsement, or implication that the entities, products, materials, or equipment are necessarily the best available for the purpose. Nor does such identification imply a finding of fault or negligence by the National Institute of Standards and Technology.

\section{Disclaimer No. 2}

The policy of NIST is to use the International System of Units (metric units) in all publications. In this document, however, units are presented in metric units or the inch-pound system, whichever is prevalent in the discipline.

\section{Disclaimer No. 3}

Pursuant to section 7 of the National Construction Safety Team Act, the NIST Director has determined that certain evidence received by NIST in the course of this Investigation is "voluntarily provided safety-related information" that is "not directly related to the building failure being investigated" and that "disclosure of that information would inhibit the voluntary provision of that type of information" (15 USC 7306c).

In addition, a substantial portion of the evidence collected by NIST in the course of the Investigation has been provided to NIST under nondisclosure agreements.

\section{Disclaimer No. 4}

NIST takes no position as to whether the design or construction of a WTC building was compliant with any code since, due to the destruction of the WTC buildings, NIST could not verify the actual (or as-built) construction, the properties and condition of the materials used, or changes to the original construction made over the life of the buildings. In addition, NIST could not verify the interpretations of codes used by applicable authorities in determining compliance when implementing building codes. Where an Investigation report states whether a system was designed or installed as required by a code provision, NIST has documentary or anecdotal evidence indicating whether the requirement was met, or NIST has independently conducted tests or analyses indicating whether the requirement was met.

\section{Use in Legal Proceedings}

No part of any report resulting from a NIST investigation into a structural failure or from an investigation under the National Construction Safety Team Act may be used in any suit or action for damages arising out of any matter mentioned in such report (15 USC 281a; as amended by P.L. 107-231).

National Institute of Standards and Technology National Construction Safety Team Act Report 1-4A Natl. Inst. Stand. Technol. Natl. Constr. Sfty. Tm. Act Rpt. 1-4A, 140 pages (September 2005) CODEN: NSPUE2

\section{U.S. GOVERNMENT PRINTING OFFICE \\ WASHINGTON: 2005}

For sale by the Superintendent of Documents, U.S. Government Printing Office

Internet: bookstore.gpo.gov — Phone: (202) 512-1800 — Fax: (202) 512-2250

Mail: Stop SSOP, Washington, DC 20402-0001 


\section{ABSTRACT}

Fires occurred in World Trade Center (WTC) 1, 2, and 7 prior to September 11, 2001. This report documents the facts of significant fires in the buildings after first occupancy as they relate to the performance of the automatic sprinkler, manual suppression, fire detection, and smoke purge systems. The ultimate goal of this review was to identify from New York City Fire Department (FDNY) records significant but not well-known fires for further study.

From the information contained in FDNY fire reports and fire investigation records provided to the National Institute of Standards and Technology (NIST), 47 fires occurred in WTC 1, 2, and 7 that were of sufficient size and duration to activate multiple sprinklers or were estimated by NIST to be capable of doing so, over the time period the buildings were occupied. This total does not include the major 1975 office fire in WTC 1 or the 1993 bombing.

The records indicate that in areas protected by automatic sprinklers, no fire activated more than three sprinklers. Three sprinklers would provide coverage for a floor area of approximately $675 \mathrm{ft}^{2}\left(63 \mathrm{~m}^{2}\right)$. This area is much smaller than the $9,000 \mathrm{ft}^{2}\left(800 \mathrm{~m}^{2}\right)$ damaged by the 1975 fire in an office space unprotected with automatic sprinklers.

Many of the fires that occurred were recorded as suspicious or unknown in cause, occurred during off peak work hours, and involved materials such as trash or paper-based supplies. In cases where sprinklers were activated, the FDNY records indicated that the sprinklers either extinguished the fire completely or aided in controlling the spread.

Keywords: Fire, fire detection, manual fire suppression, smoke purge systems, sprinklers, World Trade Center. 
This page intentionally left blank. 


\section{TABLE OF CONTENTS}

Abstract iii

List of Figures vii

List of Tables ix

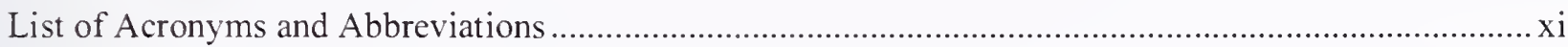

Preface xiii

\section{Chapter 1}

New York City Fire Department Records. 1

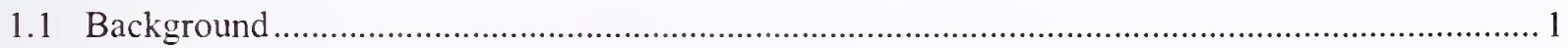

1.2 FDNY Fire Reports and Fire Investigation Reports ...................................................... 1

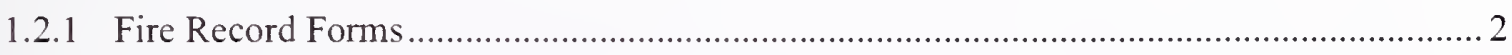

1.2.2 Overview of Fire Incidents 1970-2001 from FDNY Records ........................................ 3

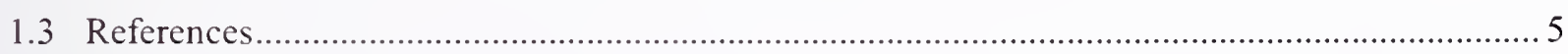

\section{Chapter 2}

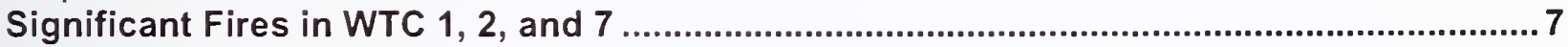

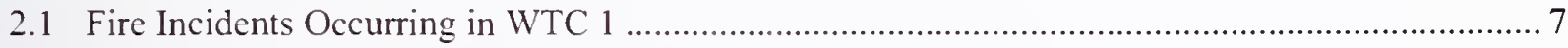

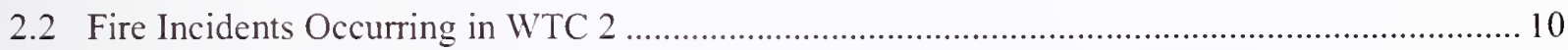

2.3 Additional Fires Involving Deployment of Standpipe Lines in WTC 1 and WTC 2............... 11

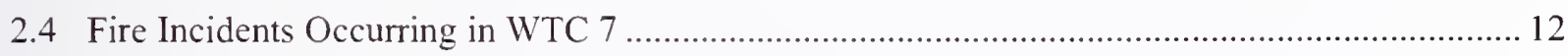

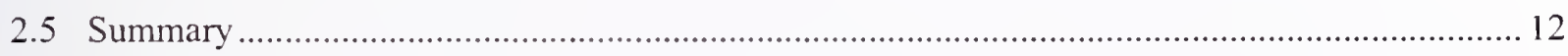

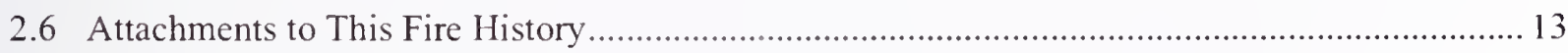

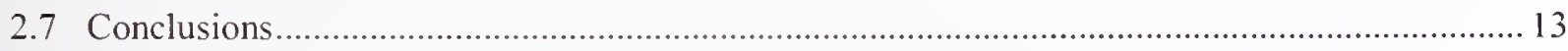

\section{Appendix A}

Explanation of Numeric Codes Prior to 1980 ................................................................. 15

\section{Appendix B}

Explanation of Numeric Codes from 1981 to 1987

Appendix C

Explanation of Numeric Codes from 1987 to Present

\section{Appendix D}

Significant Fires in WTC 1 


\section{Appendix E}

Significant Fires in WTC 2

\section{Appendix F}

Fires with Standpipes and Sprinklers in WTC 1 and WTC 2

Appendix G

Fires with Standpipes in WTC 1 and WTC 2

Appendix $\mathrm{H}$

Significant Fires in WTC 7

Appendix 1

Extracted Page from PACO Report on Sprinkler Program 


\section{LIST OF FIGURES}

Figure $\mathrm{P}-1$. The eight projects in the federal building and fire safety investigation of the WTC disaster. 
This page intentionally left blank. 


\section{LIST OF TABLES}

Table P-1. Federal building and fire safety investigation of the WTC disaster. .................................. xiv

Table P-2. Public meetings and briefings of the WTC Investigation................................................. xvii

Table 1-1. Categorization of WTC 1, 2, and 7 fires from FDNY records.......................................... 3

Table 2-1. Significant fires in WTC 1 extinguished by sprinklers and/or multiple standpipe lines........... 7

Table 2-2. Significant fires in WTC 2 extinguished by sprinklers and/or multiple standpipe lines......... 10

Table 2-3. Significant fires in WTC 7 extinguished by sprinklers and/or multiple standpipe lines.......... 12 
This page intentionally left blank. 


\section{LIST OF ACRONYMS AND ABBREVIATIONS}

\section{Acronyms}

DTAP dissemination and technical assistance program

FDNY New York City Fire Department

FEMA Federal Emergency Management Agency

HVAC heating, ventilating, and air conditioning

NIST National Institute of Standards and Technology

PANYNJ Port Authority of New York and New Jersey

R\&D research and development

WTC World Trade Center

WTC $1 \quad$ World Trade Center 1 (North Tower)

WTC 2 World Trade Center 2 (South Tower)

WTC $7 \quad$ World Trade Center 7

\section{Abbreviations}

$\mathrm{ft}^{2} \quad$ square foot

$\mathrm{m}^{2} \quad$ square meter 
This page intentionally left blank. 


\section{PREFACE}

\section{Genesis of This Investigation}

Immediately following the terrorist attack on the World Trade Center (WTC) on September 11, 2001, the Federal Emergency Management Agency (FEMA) and the American Soeiety of Civil Engineers began planning a building performance study of the disaster. The week of October 7, as soon as the rescue and search efforts ceased, the Building Performance Study Team went to the site and began its assessment. This was to be a brief effort. as the study team eonsisted of experts who largely volunteered their time away from their other professional commitments. The Building Performance Study Team issued its report in May 2002, fulfilling its goal "to determine probable failure mechanisms and to identify areas of future investigation that could lead to practical measures for improving the damage resistanee of buildings against such unforeseen events."

On August 21, 2002, with funding from the U.S. Congress through FEMA, the National Institute of Standards and Technology (NIST) announced its building and fire safety investigation of the WTC disaster. On October 1, 2002, the National Construction Safety Team Act (Public Law 107-231), was signed into law. The NIST WTC Investigation was conducted under the authority of the National Construetion Safety Team Act.

The goals of the investigation of the WTC disaster were:

- To investigate the building eonstruction, the materials used, and the technical conditions that contributed to the outcome of the WTC disaster.

- To serve as the basis for:

- Improvements in the way buildings are designed, constructed, maintained, and used;

- Improved tools and guidance for industry and safety officials;

- Recommended revisions to current codes, standards, and praetices; and

- Improved publie safety.

The specifie objectives were:

1. Determine why and how WTC 1 and WTC 2 collapsed following the initial impacts of the aircraft and why and how WTC 7 collapsed;

2. Determine why the injuries and fatalities were so high or low depending on location, ineluding all technieal aspects of fire proteetion, occupant behavior, evacuation, and emergency response;

3. Determine what procedures and practices were used in the design, construction, operation, and maintenanee of WTC 1,2, and 7; and

4. Identify, as specifically as possible, areas in current building and fire codes, standards, and practices that warrant revision. 
NIST is a nonregulatory agency of the U.S. Department of Commerce's Technology Administration. The purpose of NIST investigations is to improve the safety and structural integrity of buildings in the United States, and the focus is on fact finding. NIST investigative teams are authorized to assess building performance and emergency response and evacuation procedures in the wake of any building failure that has resulted in substantial loss of life or that posed significant potential of substantial loss of life. NIST does not have the statutory authority to make findings of fault nor negligence by individuals or organizations. Further, no part of any report resulting from a NIST investigation into a building failure or from an investigation under the National Construction Safety Team Act may be used in any suit or action for damages arising out of any matter mentioned in such report (15 USC 281a, as amended by Public Law 107-231).

\section{Organization of the Investigation}

The National Construction Safety Team for this Investigation, appointed by the then NIST Director, Dr. Arden L. Bement, Jr., was led by Dr. S. Shyam Sunder. Dr. William L. Grosshandler served as Associate Lead Investigator, Mr. Stephen A. Cauffman served as Program Manager for Administration, and Mr. Harold E. Nelson served on the team as a private sector expert. The Investigation included eight interdependent projects whose leaders comprised the remainder of the team. A detailed description of each of these eight projects is available at http://wtc.nist.gov. The purpose of each project is summarized in Table $\mathrm{P}-1$, and the key interdependencies among the projects are illustrated in Fig. $\mathrm{P}-1$.

Table P-1. Federal building and fire safety investigation of the WTC disaster.

\begin{tabular}{|c|c|}
\hline Technical Area and Project Leader & Project Purpose \\
\hline $\begin{array}{l}\text { Analysis of Building and Fire Codes and } \\
\text { Practices; Project Leaders: Dr. H. S. Lew } \\
\text { and Mr. Richard W. Bukowski }\end{array}$ & $\begin{array}{l}\text { Document and analyze the code provisions, procedures, and } \\
\text { practices used in the design, construction, operation, and } \\
\text { maintenance of the structural, passive fire protection, and } \\
\text { emergency access and evacuation systems of WTC } 1,2 \text {, and } 7 \text {. }\end{array}$ \\
\hline $\begin{array}{l}\text { Baseline Structural Performance and } \\
\text { Aircraft Impact Damage Analysis; Project } \\
\text { Leader: Dr. Fahim H. Sadek }\end{array}$ & $\begin{array}{l}\text { Analyze the baseline performance of WTC } 1 \text { and WTC } 2 \text { under } \\
\text { design, service, and abnormal loads, and aircraft impact damage on } \\
\text { the structural, fire protection. and egress systems. }\end{array}$ \\
\hline $\begin{array}{l}\text { Mechanical and Metallurgical Analysis of } \\
\text { Structural Steel; Project Leader: Dr. Frank } \\
\text { W. Gayle }\end{array}$ & $\begin{array}{l}\text { Determine and analyze the mechanical and metallurgical properties } \\
\text { and quality of steel, weldments, and connections from steel } \\
\text { recovered from WTC } 1,2 \text {, and } 7 \text {. }\end{array}$ \\
\hline $\begin{array}{l}\text { Investigation of Active Fire Protection } \\
\text { Systems; Project Leader: Dr. David } \\
\text { D. Evans; Dr. William Grosshandler }\end{array}$ & $\begin{array}{l}\text { Investigate the performance of the active fire protection systems in } \\
\text { WTC } 1,2 \text {, and } 7 \text { and their role in fire control. emergency response, } \\
\text { and fate of occupants and responders. }\end{array}$ \\
\hline $\begin{array}{l}\text { Reconstruction of Thermal and Tenability } \\
\text { Environment; Project Leader: Dr. Richard } \\
\text { G. Gann }\end{array}$ & $\begin{array}{l}\text { Reconstruct the time-evolving temperature, thermal environment, } \\
\text { and smoke movement in WTC } 1,2 \text {, and } 7 \text { for use in evaluating the } \\
\text { structural performance of the buildings and behavior and fate of } \\
\text { occupants and responders. }\end{array}$ \\
\hline $\begin{array}{l}\text { Structural Fire Response and Collapse } \\
\text { Analysis; Project Leaders: Dr. John } \\
\text { L. Gross and Dr. Therese P. McAllister }\end{array}$ & $\begin{array}{l}\text { Analyze the response of the WTC towers to fires with and without } \\
\text { aircraft damage, the response of WTC } 7 \text { in fires, the performance } \\
\text { of composite steel-trussed floor systems, and determine the most } \\
\text { probable structural collapse sequence for WTC } 1,2 \text {, and } 7 \text {. }\end{array}$ \\
\hline $\begin{array}{l}\text { Occupant Behavior, Egress, and Emergency } \\
\text { Communications; Project Leader: Mr. Jason } \\
\text { D. Averill }\end{array}$ & $\begin{array}{l}\text { Analyze the behavior and fate of occupants and responders, both } \\
\text { those who survived and those who did not, and the performance of } \\
\text { the evacuation system. }\end{array}$ \\
\hline $\begin{array}{l}\text { Emergency Response Technologies and } \\
\text { Guidelines; Project Leader: Mr. J. Randall } \\
\text { Lawson }\end{array}$ & $\begin{array}{l}\text { Document the activities of the emergency responders from the time } \\
\text { of the terrorist attacks on WTC } 1 \text { and WTC } 2 \text { until the collapse of } \\
\text { WTC 7, including practices followed and technologies used. }\end{array}$ \\
\hline
\end{tabular}




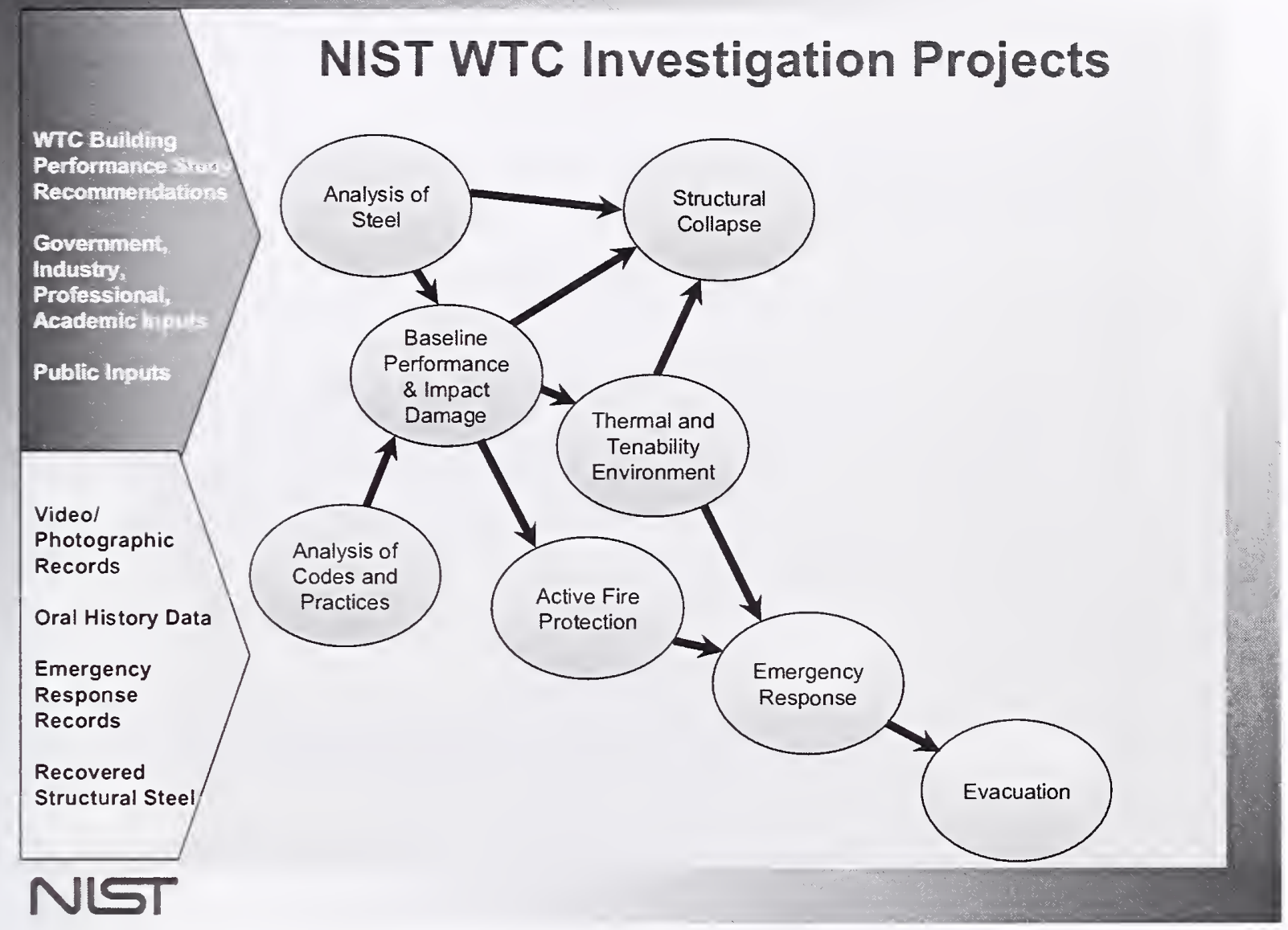

Figure P-1. The eight projects in the federal building and fire safety investigation of the WTC disaster.

\section{National Construction Safety Team Advisory Committee}

The NIST Director also established an advisory committee as mandated under the National Construction Safety Team Act. The initial members of the committee were appointed following a public solicitation. These were:

- Paul Fitzgerald, Executive Vice President (retired) FM Global, National Construction Safety Team Advisory Committee Chair

- John Barsom, President, Barsom Consulting, Ltd.

- John Bryan, Professor Emeritus, University of Maryland

- David Collins, President, The Preview Group, Inc.

- Glenn Corbett, Professor, John Jay College of Criminal Justice

- Philip DiNenno, President, Hughes Associates, Inc. 
- Robcrt Hanson, Professor Emeritus, University of Michigan

- Charles Thornton, Co-Chairman and Managing Principal, The Thornton-Tomasetti Group, Inc.

- Kathleen Tierney, Director, Natural Hazards Research and Applications Information Center, University of Colorado at Boulder

- Forman Williams, Director, Center for Energy Research, University of California at San Diego

This National Construction Safety Tcam Advisory Committee provided technical advice during the Investigation and commentary on drafts of the Investigation reports prior to their public release. NIST has benefited from the work of many people in the preparation of these reports, including the National Construction Safety Team Advisory Committee. The content of the reports and recommendations, however, are solely the responsibility of NIST.

\section{Public Outreach}

During the course of this Investigation, NIST held public briefings and meetings (listed in Table P-2) to solicit input from the public, present preliminary findings, and obtain comments on the direction and progress of the Investigation from the public and the Advisory Committee.

NIST maintained a publicly accessible Web site during this Investigation at http://wtc.nist.gov. The site contained extensive information on the background and progrcss of the Investigation.

\section{NIST's WTC Public-Private Response Plan}

The collapse of the WTC buildings has led to broad reexamination of how tall buildings are designed, constructed, maintained, and used, especially with regard to major events such as fires, natural disasters, and terrorist attacks. Reflecting the enhanced interest in effecting necessary change, NIST, with support from Congress and the Administration, has put in place a program, the goal of which is to develop and implement the standards, technology, and practices needed for cost-effective improvements to the safety and security of buildings and building occupants, including evacuation, emergency response procedures, and threat mitigation.

The strategy to meet this goal is a three-part NIST-led public-private response program that includes:

- A federal building and fire safety investigation to study the most probable factors that contributed to post-aircraft impact collapse of the WTC towers and the 47-story WTC 7 building, and the associated evacuation and emergency response experience.

- A research and development (R\&D) program to (a) facilitate the implementation of recommendations resulting from the WTC Investigation, and (b) provide the technical basis for cost-effective improvements to national building and fire codes, standards, and practices that enhance the safety of buildings, their occupants, and emergency responders. 
Table P-2. Public meetings and briefings of the WTC Investigation.

\begin{tabular}{|c|c|c|}
\hline Date & Location & Principal Agenda \\
\hline June 24,2002 & New York City, NY & $\begin{array}{l}\text { Public meeting: Public comments on the Draft Plan for the } \\
\text { pending WTC Investigation. }\end{array}$ \\
\hline August 21,2002 & Gaithersburg, MD & Media briefing announcing the formal start of the Investigation. \\
\hline December 9.2002 & Washington, DC & $\begin{array}{l}\text { Media briefing on release of the Public Update and NIST request } \\
\text { for photographs and videos. }\end{array}$ \\
\hline April 8, 2003 & New York City, NY & $\begin{array}{l}\text { Joint public forum with Columbia University on first-person } \\
\text { interviews. }\end{array}$ \\
\hline April 29-30, 2003 & Gaithersburg, MD & $\begin{array}{l}\text { NCST Advisory Committee meeting on plan for and progress on } \\
\text { WTC Investigation with a public comment session. }\end{array}$ \\
\hline May 7.2003 & New York City, NY & Media briefing on release of May 2003 Progress Report. \\
\hline August 26-27, 2003 & Gaithersburg, MD & $\begin{array}{l}\text { NCST Advisory Committee meeting on status of the WTC } \\
\text { investigation with a public comment session. }\end{array}$ \\
\hline September 17,2003 & New York City, NY & $\begin{array}{l}\text { Media and public briefing on initiation of first-person data } \\
\text { collection projects. }\end{array}$ \\
\hline December 2-3, 2003 & Gaithersburg, MD & $\begin{array}{l}\text { NCST Advisory Committee meeting on status and initial results } \\
\text { and release of the Public Update with a public comment session. }\end{array}$ \\
\hline February 12,2004 & New York City, NY & $\begin{array}{l}\text { Public meeting on progress and preliminary findings with public } \\
\text { comments on issues to be considered in formulating final } \\
\text { recommendations. }\end{array}$ \\
\hline June 18,2004 & New York City, NY & Media/public briefing on release of June 2004 Progress Report. \\
\hline June $22-23,2004$ & Gaithersburg, MD & $\begin{array}{l}\text { NCST Advisory Committee meeting on the status of and } \\
\text { preliminary findings from the WTC Investigation with a public } \\
\text { comment session. }\end{array}$ \\
\hline August 24, 2004 & Northbrook, IL & $\begin{array}{l}\text { Public viewing of standard fire resistance test of WTC floor } \\
\text { system at Underwriters Laboratories, Inc. }\end{array}$ \\
\hline October 19-20, 2004 & Gaithersburg, MD & $\begin{array}{l}\text { NCST Advisory Committee meeting on status and near complete } \\
\text { set of preliminary findings with a public comment session. }\end{array}$ \\
\hline November 22, 2004 & Gaithersburg, MD & $\begin{array}{l}\text { NCST Advisory Committee discussion on draft annual report to } \\
\text { Congress, a public comment session, and a closed session to } \\
\text { discuss pre-draft recommendations for WTC Investigation. }\end{array}$ \\
\hline April 5, 2005 & New York City, NY & $\begin{array}{l}\text { Media and public briefing on release of the probable collapse } \\
\text { sequence for the WTC towers and draft reports for the projects on } \\
\text { codes and practices, evacuation, and emergency response. }\end{array}$ \\
\hline June 23,2005 & New York City, NY & $\begin{array}{l}\text { Media and public briefing on release of all draft reports for the } \\
\text { WTC towers and draft recommendations for public comment. }\end{array}$ \\
\hline $\begin{array}{l}\text { September 12-13, } \\
2005\end{array}$ & Gaithersburg, MD & $\begin{array}{l}\text { NCST Advisory Committee meeting on disposition of public } \\
\text { comments and update to draft reports for the WTC towers. }\end{array}$ \\
\hline $\begin{array}{l}\text { September } 13-15 \text {, } \\
2005\end{array}$ & Gaithersburg, MD & $\begin{array}{l}\text { WTC Technical Conference for stakeholders and technical } \\
\text { community for dissemination of findings and recommendations } \\
\text { and opportunity for public to make technical comments. }\end{array}$ \\
\hline
\end{tabular}

- A dissemination and technical assistance program (DTAP) to (a) engage leaders of the construction and building community in ensuring timely adoption and widespread use of proposed changes to practices, standards, and codes resulting from the WTC Investigation and the R\&D program, and (b) provide practical guidance and tools to better prepare facility owners, contractors, architects, engineers, emergency responders, and regulatory authorities to respond to future disasters.

The desired outcomes are to make buildings, occupants, and first responders safer in future disaster events. 


\section{National Construction Safety Team Reports on the WTC Investigation}

A final report on the collapse of the WTC towers is being issued as NIST NCSTAR 1. A companion report on the collapse of WTC 7 is being issued as NIST NCSTAR 1A. The present report is one of a set that provides more detailed documentation of the Investigation findings and the means by which these technical results were achieved. As such, it is part of the archival record of this Investigation. The titles of the full set of Investigation publications are:

NIST (National Institute of Standards and Technology). 2005. Federal Burilding and Fire Safety Investigation of the World Trade Center Disaster: Final Report on the Collapse of the World Trade Center Towers. NIST NCSTAR 1. Gaithersburg, MD, September.

NIST (National Institute of Standards and Technology). 2006. Federal Building and Fire Safety Investigation of the World Trade Center Disaster: Final Report on the Collapse of World Trade Center 7. NIST NCSTAR 1A. Gaithersburg, MD.

Lew, H. S., R. W. Bukowski, and N. J. Carino. 2005. Federal Building and Fire Safety Investigation of the World Trade Center Disaster: Design, Constrmction, and Maintenance of Structural and Life Safety Systems. NIST NCSTAR 1-1. National Institute of Standards and Technology. Gaithersburg, MD, September.

Fanella, D. A., A. T. Derecho, and S. K. Ghosh. 2005. Federal Building and Fire Safety Investigation of the World Trade Center Disaster: Design and Construction of Structural Systenis. NIST NCSTAR 1-1A. National Institute of Standards and Technology. Gaithersburg, MD, September.

Ghosh, S. K., and X. Liang. 2005. Federal Building and Fire Safety Investigation of the World Trade Center Disaster: Comparison of Building Code Structural Requirements. NIST NCSTAR 1-1B. National Institute of Standards and Technology. Gaithersburg, MD, September.

Fanella, D. A., A. T. Derecho, and S. K. Ghosh. 2005. Federal Brilding and Fire Safety Investigation of the World Trade Center Disaster: Maintenance and Modifications to Structural Systems. NIST NCSTAR 1-1C. National Institute of Standards and Technology. Gaithersburg, MD, September.

Grill, R. A., and D. A. Johnson. 2005. Federal Building and Fire Safety Irvestigation of the World Trade Center Disaster: Fire Protection and Life Safety Provisions Applied to the Design and Construction of World Trade Center 1, 2, and 7 and Post-Construction Provisions Applied after Occupancy. NIST NCSTAR 1-1D. National Institute of Standards and Technology. Gaithersburg, MD, September.

Razza, J. C., and R. A. Grill. 2005. Federal Building and Fire Safety Investigation of the World Trade Center Disaster: Comparison of Codes, Standards, and Practices in Use at the Time of the Design and Construction of World Trade Center 1, 2, and 7. NIST NCSTAR 1-1E. National Institute of Standards and Technology. Gaithersburg, MD, September.

Grill, R. A., D. A. Johnson, and D. A. Fanella. 2005. Federal Building and Fire Safety

Investigation of the World Trade Center Disaster: Comparison of the 1968 and Current (2003) New 
York City Burilding Code Provisions. NIST NCSTAR 1-1F. National Institute of Standards and Technology. Gaithersburg, MD, September.

Grill, R. A., and D. A. Johnson. 2005. Federal Burilding and Fire Safety Investigation of the World Trade Center Disaster: Amendments to the Fire Protection and Life Safety Provisions of the New York City Building Code by Local Law's Adopted While World Trade Center 1, 2, and 7 Were in Use. NIST NCSTAR 1-1G. National Institute of Standards and Technology. Gaithersburg, MD, September.

Grill, R. A., and D. A. Johnson. 2005. Federal Building and Fire Safety Investigation of the World Trade Center Disaster: Post-Construction Modifications to Fire Protection and Life Safety Systems of World Trade Center 1 and 2. NIST NCSTAR 1-1H. National Institute of Standards and Technology. Gaithersburg, MD, September.

Grill, R. A., D. A. Johnson, and D. A. Fanella. 2005. Federal Building and Fire Safety Investigation of the World Trade Center Disaster: Post-Constuntion Modifications to Fite Protection, Life Safety, and Structural Systems of World Trade Center 7. NIST NCSTAR 1-11. National Institute of Standards and Technology. Gaithersburg, MD, September.

Grill, R. A., and D. A. Johnson. 2005. Federal Building and Fire Safety Imestigation of the World Trade Center Disaster: Design, Installation, and Operation of Fuel System for Emergency Power in World Trade Center 7. NIST NCSTAR 1-1J. National Institute of Standards and Technology. Gaithersburg, MD, September.

Sadek, F. 2005. Federal Building and Fire Safety Investigation of the World Trade Center Disaster: Baseline Structural Performance and Aircraft Impact Damage Analysis of the World Trade Center Towers. NIST NCSTAR 1-2. National Institute of Standards and Technology. Gaithersburg, MD, September.

Faschan, W. J., and R. B. Garlock. 2005. Federal Building and Fire Safety Investigation of the World Trade Center Disaster: Reference Structural Models and Baseline Performance Analysis of the World Trade Center Towers. NIST NCSTAR 1-2A. National Institute of Standards and Technology. Gaithersburg, MD, September.

Kirkpatrick, S. W., R. T. Bocchieri, F. Sadek, R. A. MacNeill, S. Holmcs, B. D. Peterson, R. W. Cilke, C. Navarro. 2005. Federal Building and Fire Safety Investigation of the World Trade Center Disaster: Analysis of Aircraft Impacts into the World Trade Center Towers, NIST NCSTAR 1-2B. National Institute of Standards and Technology. Gaithersburg, MD, September.

Gayle, F. W., R. J. Fields, W. E. Luecke, S. W. Banovic, T. Foecke, C. N. McCowan, T. A. Sicwert, and J. D. McColskey. 2005. Federal Building and Fire Safety Investigation of the World Trade Center Disaster: Mechanical and Metallurgical Analysis of Structural Steel. NIST NCSTAR 1-3. National Institute of Standards and Technology. Gaithersburg, MD, Scptember.

Luecke, W. E., T. A. Siewert, and F. W. Gayle. 2005. Federal Burilding and Fire Safety Investigation of the World Trade Center Disaster: Contemporaneous Strictural Steel Specifications. NIST Special Publication 1-3A. National Institute of Standards and Technology. Gaithersburg, MD, September. 
Banovic, S. W. 2005. Federal Building and Fire Safety Investigation of the World Trade Center Disaster: Steel Inventory and Identification. NIST NCSTAR 1-3B. National Institute of Standards and Technology. Gaithersburg, MD, September.

Banovic, S. W., and T. Foecke. 2005. Federal Building and Fire Safety Investigation of the World Trade Center Disaster: Damage and Failme Modes of Structural Steel Components. NIST NCSTAR 1-3C. National Institute of Standards and Technology. Gaithersburg, MD, September.

Luecke, W. E., J. D. McColskey, C. N. McCowan, S. W. Banovic, R. J. Fields, T. Foecke, T. A. Siewert, and F. W. Gayle. 2005. Federal Building and Fire Safety Investigation of the World Trade Center Disaster: Mechanical Properties of Structural Steels. NIST NCSTAR 1-3D. National Institute of Standards and Technology. Gaithersburg, MD, September.

Banovic, S. W., C. N. McCowan, and W. E. Luecke. 2005. Federal Building and Fire Safety Investigation of the World Trade Center Disaster: Physical Properties of Structwral Steels. NIST NCSTAR 1-3E. National Institute of Standards and Technology. Gaithersburg, MD, September.

Evans, D. D., R. D. Peacock, E. D. Kuligowski, W. S. Dols, and W. L. Grosshandler. 2005. Federal Building and Fire Safety Investigation of the World Trade Center Disaster: Active Fire Protection Systems. NIST NCSTAR 1-4. National Institute of Standards and Technology. Gaithersburg, MD, September.

Kuligowski, E. D., D. D. Evans, and R. D. Peacock. 2005. Federal Building and Fire Safety Investigation of the World Trade Center Disaster: Post-Construction Fires Prior to September I1, 2001. NIST NCSTAR 1-4A. National Institute of Standards and Technology. Gaithersburg, MD, September.

Hopkins, M., J. Schoenrock, and E. Budnick. 2005. Federal Building and Fire Safety Investigation of the World Trade Center Disaster: Fire Suppression Systems. NIST NCSTAR 1-4B. National Institute of Standards and Technology. Gaithersburg, MD, September.

Keough, R. J., and R. A. Grill. 2005. Federal Building and Fire Safety Investigation of the World Trade Center Disaster: Fire Alarm Systems. NIST NCSTAR 1-4C. National Institute of Standards and Technology. Gaithersburg, MD, September.

Ferreira, M. J., and S. M. Strege. 2005. Federal Building and Fire Safety Investigation of the World Trade Center Disaster: Smoke Management Systems. NIST NCSTAR 1-4D. National Institute of Standards and Technology. Gaithersburg, MD, September.

Gann, R. G., A. Hamins, K. B. McGrattan, G. W. Mulholland, H. E. Nelson, T. J. Ohlemiller, W. M. Pitts, and K. R. Prasad. 2005. Federal Building and Fire Safety Investigation of the World Trade Center Disaster: Reconstruction of the Fires in the World Trade Center Towers. NIST NCSTAR 1-5. National Institute of Standards and Technology. Gaithersburg, MD, September.

Pitts, W. M., K. M. Butler, and V. Junker. 2005. Federal Building and Fire Safety Investigation of the World Trade Center Disaster: Visual Evidence, Damage Estimates, and Timeline Analysis. NIST NCSTAR 1-5A. National Institute of Standards and Technology. Gaithersburg, MD, September. 
Hamins, A., A. Maranghides, K. B. McGrattan, E. Johnsson, T. J. Ohlemiller, M. Donnelly, J. Yang, G. Mulholland, K. R. Prasad, S. Kukuck, R. Anleitner and T. McAllister. 2005. Federal Bullding and Fire Safety Investigation of the World Trade Center Disaster: Experinents and Modeling of Strnctural Steel Elements Exposed to Fire. NIST NCSTAR 1-5B. National Institute of Standards and Technology. Gaithersburg, MD, September.

Ohlemiller, T. J., G. W. Mulholland, A. Maranghides, J. J. Filliben, and R. G. Gann. 2005. Federal Building and Fire Safety Investigation of the World Trade Center Disaster: Fire Tests of Single Office Workstations. NIST NCSTAR 1-5C. National Institute of Standards and Technology. Gaithersburg, MD, September.

Gann, R. G., M. A. Riley, J. M. Repp, A. S. Whittaker, A. M. Reinhorn, and P. A. Hough. 2005. Federal Building and Fire Safety Investigation of the World Trade Center Disaster: Reaction of Ceiling Tile Systems to Shocks. NIST NCSTAR 1-5D. National Institute of Standards and Technology. Gaithersburg, MD, September.

Hamins. A., A. Maranghides, K. B. McGrattan, T. J. Ohlemiller, and R. Anleitner. 2005. Federal Building and Fire Safety Investigation of the World Trade Center Disaster: Experiments and Modeling of Multiple Workstations Burning in a Conupartunent. NIST NCSTAR 1-5E. National Institute of Standards and Technology. Gaithersburg, MD, September.

McGrattan, K. B., C. Bouldin, and G. Forney. 2005. Federal Bullding and Fire Safety Investigation of the World Trade Center Disaster: Computer Simnlation of the Fires in the World Trade Center Towers. NIST NCSTAR 1-5F. National Institute of Standards and Technology. Gaithersburg, MD, September.

Prasad, K. R., and H. R. Baum. 2005. Federal Building and Fire Safety Investigation of the World Trade Center Disaster: Fire Strncture Interface and Thermal Response of the World Trade Center Towers. NIST NCSTAR 1-5G. National Institute of Standards and Technology. Gaithersburg, MD, September.

Gross, J. L., and T. McAllister. 2005. Federal Building and Fire Safety Investigation of the World Tiade Center Disaster: Strnctural Fire Response and Probable Collapse Sequence of the World Trade Center Towers. NIST NCSTAR 1-6. National Institute of Standards and Technology. Gaithersburg, MD, September.

Carino, N. J., M. A. Starnes, J. L. Gross, J. C. Yang, S. Kukuck, K. R. Prasad, and R. W. Bukowski. 2005. Federal Building and Fire Safety Investigation of the World Trade Center Disaster: Passive Fire Protection. NIST NCSTAR 1-6A. National Institute of Standards and Technology. Gaithersburg, MD, September.

Gross, J., F. Hervey, M. Izydorek, J. Mammoser, and J. Treadway. 2005. Federal Building and Fire Safety Investigation of the World Trade Center Disaster: Fire Resistance Tests of Floor Tituss Systems. NIST NCSTAR 1-6B. National Institute of Standards and Technology. Gaithersburg, MD, September.

Zarghamee, M. S., S. Bolourchi, D. W. Eggers, Ö. O. Erbay, F. W. Kan, Y. Kitane, A. A. Liepins, M. Mudlock, W. I. Naguib, R. P. Ojdrovic, A. T. Sarawit, P. R Barrett, J. L. Gross, and 
T. P. McAllister. 2005. Federal Building and Fire Safety Investigation of the World Trade Center Disaster: Component, Connection, and Subsystem Structural Analysis. NIST NCSTAR 1-6C. National Institute of Standards and Technology. Gaithersburg, MD, September.

Zarghamee, M. S., Y. Kitane, Ö. O. Erbay, T. P. McAllister, and J. L. Gross. 2005. Federal Building and Fire Safety Investigation of the World Trade Center Disaster: Global Strnctural Analysis of the Response of the World Trade Center Towers to Impact Damage and Fire. NIST NCSTAR 1-6D. National Institute of Standards and Technology. Gaithersburg, MD, September.

McAllister, T., R. W. Bukowski, R. G. Gann, J. L. Gross, K. B. McGrattan, H. E. Nelson, L. Phan, W. M. Pitts, K. R. Prasad, F. Sadek. 2006. Federal Building and Fire Safety Investigation of the World Trade Center Disaster: Structural Fire Response and Probable Collapse Sequence of World Trade Center 7. (Provisional). NIST NCSTAR 1-6E. National Institute of Standards and Technology. Gaithersburg, MD.

Gilsanz, R., V. Arbitrio, C. Anders, D. Chlebus, K. Ezzeldin, W. Guo, P. Moloney, A. Montalva, J. Oh, K. Rubenacker. 2006. Federal Building and Fire Safety Investigation of the World Trade Center Disaster: Structural Analysis of the Response of World Trade Center 7 to Debris Damage and Fire. (Provisional). NIST NCSTAR 1-6F. National Institute of Standards and Technology. Gaithersburg, MD.

Kim, W. 2006. Federal Building and Fire Safety Investigation of the World Trade Center Disaster: Analysis of September 11, 2001, Seismogram Data. (Provisional). NIST NCSTAR 1-6G. National Institute of Standards and Technology. Gaithersburg, MD.

Nelson, K. 2006. Federal Building and Fire Safety Investigation of the World Trade Center Disaster: The Con Ed Substation in World Trade Center 7. (Provisional). NIST NCSTAR 1-6H. National Institute of Standards and Technology. Gaithersburg, MD.

Averill, J. D., D. S. Mileti, R. D. Peacock, E. D. Kuligowski, N. Groner, G. Proulx, P. A. Reneke, and H. E. Nelson. 2005. Federal Building and Fire Safety Imvestigation of the World Trade Center Disaster: Occupant Behavior, Egress, and Emergency Communication. NIST NCSTAR 1-7. National Institute of Standards and Technology. Gaithersburg, MD, September.

Fahy, R., and G. Proulx. 2005. Federal Building and Fire Safety Investigation of the World Trade Center Disaster: Analysis of Published Accounts of the World Trade Center Evacuation. NIST NCSTAR 1-7A. National Institute of Standards and Technology. Gaithersburg, MD, September.

Zmud, J. 2005. Federal Building and Fire Safety Investigation of the World Trade Center Disaster: Technical Documentation for Survey Administration. NIST NCSTAR 1-7B. National Institute of Standards and Technology. Gaithersburg, MD, September.

Lawson, J. R., and R. L. Vettori. 2005. Federal Building and Fire Safety Investigation of the World Trade Center Disaster: The Emergency Response Operations. NIST NCSTAR 1-8. National Institute of Standards and Technology. Gaithersburg, MD, September. 


\section{Chapter 1 \\ NEW YORK CITY FIRE DEPARTMENT RECORDS}

Fires occurred in World Trade Center (WTC) 1, 2, and 7 prior to September 11, 2001. This report documents the facts of significant fires in the buildings after first occupancy as they relate to the performance of the automatic sprinkler, manual suppression, fire detection, and smoke purgc systems. The ultimate goal of this review was to identify from New York City Fire Department (FDNY) records significant but not well known fires for further study.

\section{$1.1 \quad$ BACKGROUND}

The fire protection engineering department of the Port Authority of New York and New Jersey (PANYNJ) maintained records of all significant fire events in the WTC buildings. These records were lost in the collapse of the towers.

Two significant fire events involving WTC 1 are well known. On February 14, 1975, a fire started on floor 11 of WTC 1. Workers reported the fire to WTC police headquarters. When police reached the fire floor, they reported a serious fire and ordered the heating, ventilating, and air conditioning (HVAC) system be placed into the smoke purge mode. Fire spread through unprotected floor openings in utility closets. Fire damage occurred on floor 10 through floor 19. Approximately $9,000 \mathrm{ft}^{2}\left(800 \mathrm{~m}^{2}\right)$ of the floor 11 contents were destroyed or damaged. At that time, sprinklers had not been installed in the office spaces. However, fire barriers divided the floor into quadrants. The fire on floor 11 was confined to the southeast quadrant. Fire damage on other floors was confined to the utility closets. The fire was extinguished by the FDNY. More details about this fire incident can be found in Powers (1975) and Lathrop (1975).

At 12:18 p.m. on Fcbruary 26, 1993, a bomb exploded in an underground parking garagc of the WTC complex. The explosion occurred on the B2 lcvel in the area of the garage under WTC 3 and adjacent to WTC 1. The explosion resulted in a loss of normal electric powcr in WTC 1 and WTC 2. HVAC systems shut down. Smoke spread throughout WTC 1 and to a lesser cxtent in WTC 2. More details about this fire can be found in Isner and Klein (1993a, 1993b). The only historic record of smaller fire incidents in WTC 1, 2, and 7 known to this investigation are the fire reports and fire investigation reports prepared by the FDNY. These reports were provided to NIST by the FDNY for use in this Investigation.

\section{$1.2 \quad$ FDNY FIRE REPORTS AND FIRE INVESTIGATION REPORTS}

The FDNY released 397 Bureau of Operations Fire Reports and 112 Bureau of Fire Investigation Records (Fire Marshals' Reports), which served as the basis for a summary of the fire history in WTC 1, 2, and 7. The National Institute of Standards and Technology (NIST) obtained reports of fircs for the period of 1970-2001 and fire investigation records between 1977 and 2001 for WTC 1, 2, and 7, which in total, consisted of over 500 documents on which to report. These records included all responses to fires in WTC 1, 2, and 7 by the FDNY. All of these records consist of standardized forms that may be 
supplemented with other materials. Many were for minor fire events, such as fires that were extinguished by occupants before FDNY arrival. These were not of intcrest for this investigation. The records of significant fires wcre identified.

Significant fire incidents were those that exercised the fire suppression systems, specifically multiple sprinklers or multiple standpipes (with or without the activation of at least onc sprinkler). These fires will be discussed individually, organized by the building in which they occurred. In addition to these fires, generalized facts relating to those fires involving the use of one standpipe line and one sprinkler and the use of one standpipe line will be provided throughout this report. Appendixcs A through $\mathrm{H}$ of this report are explanation of numeric codes and reproductions of relevant firc reports. As an aside, the majority of fire records for significant fires documented the performance of the detectors and sprinkler systems, but almost all reports lacked information about the performance of the smoke purge system.

\subsubsection{Fire Record Forms}

Depending upon the type and date of the incident, a specific fire report form was used by the FDNY to documcnt the incident. For each type of emergency responded to by the FDNY, responders either completed a form that would describe a structural fire $(\mathrm{BF}-24)$ or a form that would describe any other type of emergency $(\mathrm{BF}-25)$, such as a nonstructural fire, transportation fire, and/or any other nonfire emergency. For this historical summary, only those events logged and organized under the structural fire form, 345 documents total, were of interest and used. A structural fire form is a one-page document (unless additional information is recorded on separate sheets) that gives valuable information about the fire event on various subjects, including:

- Alarm-the date and time of the received alarm

- Injuries and casualties-the numbers of each for the incident

- Extinguishment - details of the sprinkler and standpipe performance

- Ignition-information on the equipment involved in ignition, the form of the ignition source, the material type and form that was ignited, and the ignition factor (cause)

- Structure-information on the class of construction, the use of the building, and its status (vacant, occupied, under construction, etc.)

- Fire origin-the fire location and classification

- Fire extension-the means of fire extension and number of buildings/vehicles involved

- Damage-information on the damage done by flame, smoke, and water

- Detectors - the type, power source, and performance of the detectors in the fire area

Each subject of the incident is given a set of codes or numbers that correspond to any incident, and in order to read the fire records successfully, an understanding of the codes is necessary (see Appendixes A, 
$\mathrm{B}$, and $\mathrm{C})$. For the nonstructural B-25 record forms, the only fire-related subjects included are the injury and casualty numbers, ignition. and structure information.

Depending upon the date of the fire incident, certain information is lacking from the structural fire form. Before 1980, a different record form for structural fire incidents was used that left out the following subjects: fire extension, damage, detectors, and portions of the ignition data. Because of this, detection data are not available for the majority of the fires occurring before 1980 .

\subsubsection{Overview of Fire Incidents 1970-2001 from FDNY Records}

Table 1-1 contains the categorization of all structural fire incidents contained in the FDNY records for WTC 1, 2, and 7 available to this investigation. The table contains information on the category of fire incident (whether or not the detection and/or sprinkler systems activated), the time period over which the fires occurred, the numbers of records in that category, and a descriptive statement about the category.

Table 1-1. Categorization of WTC 1, 2, and 7 fires from FDNY records.

\begin{tabular}{|c|c|c|c|}
\hline Category & Dates & Number & Generalization of Incidents \\
\hline \multicolumn{4}{|c|}{ WTC 1} \\
\hline $\begin{array}{l}\text { No detection, no } \\
\text { sprinkler }\end{array}$ & $1980-2001$ & 66 & $\begin{array}{l}\text { Unattended food/appliances, overheated elevator } \\
\text { equipment, discarded material, welding } \\
\text { operations, electrical failure and suspicious fires }\end{array}$ \\
\hline $\begin{array}{l}\text { No detection } \\
\text { information and no } \\
\text { sprinklers }\end{array}$ & $1970-1979$ & 79 & $\begin{array}{l}\text { Trash can fires, discarded material, food on stove, } \\
\text { electrical failure, overheated equipment }\end{array}$ \\
\hline Detection, no sprinklers & $1980-2000$ & 57 & $\begin{array}{l}\text { Unattended food/appliances, overheated elevator } \\
\text { equipment, discarded material, welding } \\
\text { operations, electrical failure }\end{array}$ \\
\hline Detection and sprinklers & 1977-1999 & 18 & Suspicious, electrical failure, discarded material \\
\hline \multicolumn{4}{|c|}{ WTC 2} \\
\hline $\begin{array}{l}\text { No detection, no } \\
\text { sprinkler }\end{array}$ & $1980-1999$ & 37 & $\begin{array}{l}\text { Discarded material, welding too close, overheated } \\
\text { equipment, suspicious, elevator motor }\end{array}$ \\
\hline $\begin{array}{l}\text { No detection } \\
\text { information and no } \\
\text { sprinklers }\end{array}$ & $1975-1979$ & 40 & $\begin{array}{l}\text { Discarded material, fire in office furniture, trash } \\
\text { can fires }\end{array}$ \\
\hline Detection, no sprinklers & $1981-1999$ & 40 & $\begin{array}{l}\text { Food on stove, small elevator fire, electrical } \\
\text { failure, suspicious, overheated equipment }\end{array}$ \\
\hline Detection and sprinklers & $1977-2000$ & 5 & Mechanical failure, suspicious \\
\hline \multicolumn{4}{|c|}{ WTC 7} \\
\hline $\begin{array}{l}\text { No detection, no } \\
\text { sprinkler }\end{array}$ & 2000 & 1 & Trash can fire/discarded material \\
\hline Detection, no sprinklers & 1990 & 1 & Electrical switch on floor - explosion \\
\hline Detection and sprinklers & 1988 & 1 & Suspicious \\
\hline
\end{tabular}


All FDNY records provided to NIST, unless the records were not readable, containcd relevant information about the type and performance of the suppression system. Because of this, reports of incidents in which the sprinkler system activatcd can range from 1970 to 2001 . When the table lists "[detection]" in brackcts, this is meant to symbolize that either detection was present or no information on detector performance was included on the form (as is the case with the older records). An attempt was made to compare all investigation records with the fire reports, especially those which activated the suppression system. Looking at the records in Table 1-1, it is clear that only 24 fires activated the sprinkler system from 1970-2001 from all three buildings. Many of the other structural fires without sprinkler activation were labelcd as suspicious, trash can fires, elcctrical failures, unattended food/appliances, or overheated equipment.

In order to report on significant structural fires occurring in WTC 1,2, and 7, the FDNY records had to be reviewed for those incidents that activated sprinklers, detectors, or were extinguished by hose line and those smaller fires that self-extinguished or could be extinguished using a fire extinguisher. The structural fire incidents without detection information (before 1980), had to be reviewed to locate any fires that activated the sprinkler system.

The retrofit installation of sprinklers into WTC buildings 1 and 2 was accomplished in two phases. During the first phase in 1976, sprinkler risers/mains were installed throughout WTC 1 and WTC 2. Sprinklers were installed to protect corridors, storage rooms, lobbies, and certain tenant/PANYNJ spaces. In the second phase of the retrofit from 1983 to 2001, sprinklers were installed in all remaining places in the complex (PACO 2002; shown in Appendix I). Prior to the retrofit, only the sub-grade areas and selected hazard areas wcre protected by automatic sprinklers. This retrofit, proceeded throughout the buildings as much as practical when other renovations of the office spaces were under way, such as when change of tenants occurred.

After the installation of the sprinkler risers in 1976, tenants had the option of providing sprinklers or compartmentation for fire protection in compliance with Local Law 5. It was therefore possible that during the period of time when retrofit installation of sprinklers was under way, a fire that occurred may or may not have been in an area protected by automatic sprinklers.

The forms used by the FDNY after 1987 give a detailed description of the event and whether or not a system was present at the time of the fire; however, a fire recorded before 1987 will give data only on the number of sprinklers opened. Because of this, an effort was made to look through all reports, especially those that mentioned detection performance, in order to identify fires involving the use of standpipe lines by the FDNY as an alternate indication of a significant fire.

The next section of the report will highlight significant fires occurring in WTC 1,2, and 7 . The significant fires will be described individually by WTC building, and organized by the date on which they occurred in the building. In addition to these significant fires, (1) the fires that activated one sprinkler head and involved the use of one standpipe and (2) the fires that involved the use of only one standpipe, due to the number of incidents, will be generalized as to the nature of the incidents and the procedures followed by the FDNY. 


\section{$1.3 \quad$ REFERENCES}

Isner, M. S., and T. J. Klem. 1993a. Explosion and fire disrupt World Trade Center. NFPA Journal. National Fire Protection Association. 91-104.

Isner, M. S., and T. J. Klem. 1993b. World Trade Center Explosion and Fire, New York, New York, February 26, 1993. Fire Investigation Report. National Fire Protection Association. Quincy, MA.

Lathrop, J. K. 1975. "World Trade Center fire, New York, New York." Fire Journal. July.

PACO Group. 2002. World Trade Center General Description of All Building Systents and the Capital Program. August.

Powers, W. R. 1975. One World Trade Center Fire, New York, N.Y., February 13, 1975. National Fire Protection Association. Boston, MA. 1-15. 
This page intentionally left blank. 


\section{Chapter 2 \\ SIGNIFICANT FIRES IN WTC 1, 2, AND 7}

\section{$2.1 \quad$ FIRE INCIDENTS OCCURRING IN WTC 1}

After reviewing all the New York City Fire Department (FDNY) records of fire incidents in World Trade Center 1 (WTC 1) since 1970, the significant fires were selected. There were 12 significant fires found for WTC 1, and the fire reports are included in Appendix D. Table 2-1 provides a summary of the fire incident information from the FDNY records, which is followed by individual paragraphs about each incident.

Table 2-1. Significant fires in WTC 1 extinguished by sprinklers and/or multiple standpipe lines.

\begin{tabular}{|c|c|c|c|c|c|c|}
\hline $\begin{array}{l}\text { Significant } \\
\text { Fire }\end{array}$ & $\begin{array}{l}\text { Incident } \\
\text { Date }\end{array}$ & Fire Location & $\begin{array}{l}\text { No. of } \\
\text { Sprinklers } \\
\text { Activated }\end{array}$ & $\begin{array}{l}\text { No. of } \\
\text { Standpipes } \\
\text { Used }\end{array}$ & $\begin{array}{l}\text { Cause of } \\
\text { Fire }\end{array}$ & Material Ignited \\
\hline 1 & $9 / 9 / 77$ & B-6 level storage room & 2 & 0 & None listed & Not listed \\
\hline 2 & $9 / 23 / 77$ & Dumpster on B-4 level & 2 & 0 & Not classified & Trash/waste \\
\hline 3 & $10 / 16 / 81$ & Floor 19 office area & - & 2 & $\begin{array}{l}\text { Discarded } \\
\text { material }\end{array}$ & Furniture \\
\hline 4 & $12 / 23 / 83$ & $\begin{array}{l}2 \text { dumpsters on B-4 } \\
\text { level }\end{array}$ & 2 & 1 & Suspicious & Trash/waste \\
\hline 5 & $1 / 27 / 85$ & $\begin{array}{l}\text { Office space on } \\
\text { mezzanine level (floor } \\
\text { 2) }\end{array}$ & 2 & 1 & Incendiary & Trash/waste \\
\hline 6 & $9 / 10 / 85$ & $\begin{array}{l}\text { Garbage dumpster in } \\
\text { service elevator lobby } \\
\text { on floor } 43\end{array}$ & 2 & 1 & Suspicious & Trash/waste \\
\hline 7 & $11 / 1 / 85$ & $\begin{array}{l}\text { Storage closet on B-4 } \\
\text { level }\end{array}$ & 3 & 1 & Suspicious & Supplies/stock \\
\hline 8 & $6 / 7 / 86$ & $\begin{array}{l}\text { Dumpster fire on floor } \\
106, \text { compactor room } \\
\text { on floor } 107\end{array}$ & 2 & 1 & None listed & Trash/waste \\
\hline 9 & 9/30/91 & Office on B-4 level & $\geq 1$ & 2 & $\begin{array}{l}\text { Discarded } \\
\text { material }\end{array}$ & Trash/waste \\
\hline 10 & $11 / 19 / 91$ & $\begin{array}{l}\text { Electrical closet on } \\
\text { floor } 93\end{array}$ & 0 & 2 & Short circuit & $\begin{array}{l}\text { Electrical wire or } \\
\text { cable insulation }\end{array}$ \\
\hline 11 & $7 / 23 / 92$ & $\begin{array}{l}\text { Level B-5 at the power } \\
\text { distribution panel }\end{array}$ & 0 & 2 & $\begin{array}{l}\text { Electrical } \\
\text { failure }\end{array}$ & $\begin{array}{l}\text { Electrical wire or } \\
\text { cable insulation }\end{array}$ \\
\hline 12 & $11 / 10 / 99$ & $\begin{array}{l}\text { Computer room on } \\
\text { floor } 104\end{array}$ & 3 & $\geq 1$ & None listed & $\begin{array}{l}\text { Plastics, } \\
\text { electronic equip }\end{array}$ \\
\hline
\end{tabular}

Key: $\geq$ symbol denotes that at least one of the units of the suppression system was used (and not specifically identified by the fire report); - indicates that the report acknowledges 0 sprinklers open; however, due to the date of the fire, the space may not have had a sprinkler system installed. 


\section{$\underline{\text { Significant Fire \#1 }}$}

On September 9, 1977, at 11:04 p.m., the FDNY received an alarm for a fire in the B-6 level storage room at the address of WTC 1. The fire activated two sprinklers, and was noted to be extinguished before the FDNY's arrival.

\section{$\underline{\text { Significant Fire \#2 }}$}

Another fire occurred on September 23, 1977, at 11:48 p.m., in a dumpster on the B-4 level of WTC 1. This fire also activatcd two sprinklers, and the FDNY noted that the fire had becn extinguished prior to their arrival.

In both cases, no injuries or casualties resulted from these fires, and the damage was confined to the area of origin.

\section{Significant Fire \#3}

Six years later, on October 16,1981, at 7:12 p.m., a fire occurred on floor 19 of WTC 1 . The FDNY noted that they used two standpipe lines to extinguish the fire and that one person was evacuated from the scene. Again, the fire report notes that no sprinklers opened, but does not note whether or not sprinklers were present at the time of the fire. Given the date of the incident, sprinklers are not expected to be located on floor 19. The fire was caused by discarded material and involved furniture in an office area of the floor.

\section{$\underline{\text { Significant Fire \#4 }}$}

Six years later on December 23, 1983, at 2:50 a.m., the FDNY responded to an alarm of fire and heavy smoke conditions on the B-4 level of WTC 1. The FDNY found two dumpsters fully involved in separate locations on the same floor and noted that the two activated sprinklcrs extinguished a major portion of the fire. The FDNY extinguished the rest of the flames by stretching hose from the standpipe system. Again, no injuries or casualties resulted from this fire. The cause noted on the report was suspicious and the damage was confined to the origin of the fire.

\section{$\underline{\text { Significant Fire \#5 }}$}

On January 27, 1985, at 8:53 p.m., the FDNY was called for a fire located in an unoccupied office on the mezzanine level of WTC 1. Two sprinklers contained the incendiary (involving arson) fire consuming trash paper/waste. When the FDNY arrived, they cxtinguished the remaining fire with one standpipe line. Building and content damage was confined to less than 15 percent of the space. Also, no injuries or casualties were reported.

\section{$\underline{\text { Significant Fire \#6 }}$}

Eight months later on September 10, 1985, at 4:05 p.m., the Port Authority Police informed the FDNY on arrival of a sprinkler flow and smoke condition on floor 43. A medium smoke condition was report by the FDNY on floor 43, where a fire was extinguished by two sprinklers. The fire report notes the use of one standpipe line; however, this was used during the overhaul process. This fire originated suspiciously in a garbage dumpster in a service elevator lobby. There was no building or content damage as well as no injuries or casualties reported. 


\section{$\underline{\text { Significant Fire } \# 7}$}

On November 1, 1985, at 4:05 a.m., the FDNY was called for another suspicious fire producing heavy smoke on the B-4 level under WTC 1 and WTC 2. This fire occurred in a storage closet of the men's bathroom, and the FDNY noted that three sprinklers activated to keep the fire under control until their arrival. Upon arrival, the FDNY extinguished the remaining fire in the closet area with one standpipe line. Again, the damage was noted to be confined to the area of origin.

\section{$\underline{\text { Significant Fire } \# 8}$}

Less than a year later, on June 7, 1986, at 9:49 a.m., the FDNY received an alarm for a heavy smoke condition on floor 110. For this call, fires were burning in two separate places: a garbage dumpster on floor 106 and the compactor room on floor 107. Sprinklers were noted in operation in both locations and seemed to control the fires. until the FDNY could complete extinguishment with one standpipe line on floor 106. There was no report of injuries or casualties for the previous two fires.

\section{Significant Fire \#9}

An additional fire occurred in WTC 1 where multiple standpipe lines were used along with the activation of the sprinkler system. This fire occurred on September 30, 1991, at 6:32 p.m., in an office on the B-4 level. The fire report noted that the sprinkler system operated; however, there is no mention of how many sprinklers or even their activation in the Operations/Comments section of the report. Two $13 / 4$ in. or larger hose lines were used by the FDNY to extinguish this fire. The cause of the fire was abandoned material (cigarette) igniting boxes/carton material in an office. The fire damage was confined to the area of origin and smoke damage was confined to the floor. There was one uniformed officer injured and no civilian injuries or casualties.

\section{$\underline{\text { Significant Fire } \# 10}$}

A fire occurred on November 19, 1991, at 6:27 p.m., and two 2 1/2 in. standpipe hose lines were used by the FDNY. The FDNY responded to WTC 1 for this fire due to a report of fire and smoke condition in electrical closets on possibly four floors (floors 93-96) and an alarm transmitted from floors 93 through 98. According to the fire report, the sprinklers were in service, but did not operate for this fire. The noted cause of this fire was a short circuit and the material that was ignited was electrical wire or cable insulation. The fire and smoke damage was confined to its area of origin (electrical closet). Two occupants were removed from stalled elevators during this incident, and occupants were evacuated from the scene, although an exact number is not given. Also, two occupants were injured and required first aid.

\section{$\underline{\text { Significant Fire \#11 }}$}

The FDNY responded to WTC 1 on July 23, 1992, at 10:02 p.m., due to a transformer fire on the 5th subbasement level. Firefighters found a fire situation in a large power distribution panel, where a firefighter was knocked unconscious by a shock blast from the panel. Similar to the fire in Novembcr 1991, two $21 / 2$ in. standpipe hose lines were used by the FDNY on this fire. The cause of the fire was an electrical failure and the material ignited was electrical wire or cable insulation. No appreciable damage is noted. As mentioned earlier, one firefighter was injured as well as three civilians.

\section{$\underline{\text { Significant Fire \#12 }}$}

The final fire associated with WTC 1 was one that occurred on November 10, 1999, at 11:01 p.m., in a computer room on floor 104. The FDNY noted that the fire was "knocked down" by three sprinklers 
when they arrived and they completed extinguishment with a line cxtended from the standpipc. The flame damage was confined to the area of origin and computer equipment was involved in fueling the fire. There was one injury and no casualties reported in the FDNY record for this fire.

Table 2-1 presents the 12 significant fires in WTC 1. Five of the 12 fires occurred on the basement levels and two occurred on the upper levels (above floor 100). The causes of these significant fires include suspicious, discarded materials, and electrical failures.

\section{$2.2 \quad$ FIRE INCIDENTS OCCURRING IN WTC 2}

Table 2-2 presents the significant fire occurring in WTC 2. There were three significant fires found for WTC 2, and the fire reports are included in Appendix E. Table 2-2 provides a summary of the fire incident information from FDNY records, which is followed by individual paragraphs about each incident.

Table 2-2. Significant fires in WTC 2 extinguished by sprinklers and/or multiple standpipe lines.

\begin{tabular}{|c|c|l|c|c|c|c|}
\hline $\begin{array}{c}\text { Significant } \\
\text { Fire }\end{array}$ & $\begin{array}{c}\text { Incident } \\
\text { Date }\end{array}$ & Fire Location & $\begin{array}{c}\text { Number of } \\
\text { Sprinklers } \\
\text { Activated }\end{array}$ & $\begin{array}{c}\text { Number of } \\
\text { Standpipes } \\
\text { Used }\end{array}$ & $\begin{array}{c}\text { Cause of } \\
\text { Fire }\end{array}$ & $\begin{array}{c}\text { Material } \\
\text { Ignited }\end{array}$ \\
\hline 1 & $5 / 19 / 75$ & Floor 32 & - & 3 & Incendiary & Trash/waste \\
\hline 2 & $4 / 12 / 77$ & $\begin{array}{l}\text { Ductwork over } \\
\text { grill in } \\
\text { restaurant on } \\
\text { floor 107 }\end{array}$ & 2 & 0 & None listed & Ductwork \\
\hline 3 & $3 / 22 / 93$ & $\begin{array}{l}\text { Fan motor room } \\
\text { on floor 108 }\end{array}$ & 2 & 0 & $\begin{array}{c}\text { Mechanical } \\
\text { failure }\end{array}$ & $\begin{array}{c}\text { Not } \\
\text { classified }\end{array}$ \\
\hline
\end{tabular}

Key: - Indicates that the report acknowledges 0 sprinklers open; however, due the date of the fire, the space may not have had a sprinkler system installed.

\section{$\underline{\text { Significant Fire \#1 }}$}

A fire occurred on May 19, 1975, at 9:38 p.m., on floor 32 of WTC 2. The FDNY noted that they used three standpipe lines to extinguish the fire and that the Port Authority reported occupants trapped on floors 31 and 32. The fire report notes that no sprinklers opened, but does not note whether or not sprinklers were present at the time of the fire. Given the date of the incident, sprinklers are not expected to be located on floors 31 and 32. The fire was labeled as incendiary and involved trash/waste. The FDNY stated that the fire involved the core area of the floor and was confined to that area. More than 20 people (civilians and uniformed personnel) were injured by this incident.

\section{$\underline{\text { Significant Fire \#2 }}$}

On April 4, 1977, at 1:15 p.m., the FDNY was called to WTC 2 for a fire in the ductwork over the grills in a restaurant on floor 107. The FDNY record on this fire noted that the fire was extinguished prior to its arrival. The damage was confined to the area of origin, and the fire caused no injuries or casualties.

\section{$\underline{\text { Significant Fire \#3 }}$}

The second fire occurred on March 22, 1993, at 8:39 a.m., and caused a smoke condition on floor 108 . The fire activated two sprinklers due to an overheated bearing in a fan motor room on floor 108 . The 
damage to the area did not exceed 15 percent of the space, and there were no injuries or casualties reported.

Table 2-2 presents the three significant fircs in WTC 2. No fires were discovered in WTC 2 where multiple sprinklers or standpipes were used with another suppression system. Two of the three fires occurred on the upper levels (above floor 100) and the other occurred on floor 32. The causes of these significant fires included incendiary and mechanical failures.

\subsection{ADDITIONAL FIRES INVOLVING DEPLOYMENT OF STANDPIPE LINES IN WTC 1 AND WTC 2}

The fires described in this section (31 in total) involve the use of one standpipe, with and without the activation of one sprinkler for WTC 1 and WTC 2. Four of the 31 reports describe fires that were extinguished with one sprinkler and one standpipe line (see Appendix F). Three of these fircs were located in WTC 1 between the years of 1986-1991 and the other in WTC 2 in 1981. Two of these fires occurred in basement levels, one occurred on floor 106 of WTC 1, and the last on floor 5 in WTC 1. In some of the fire rcports, the FDNY noted that the sprinkler controlled the fire, and the standpipe was used to actually extinguish the remaining fire. Half of the fires were labeled as incendiary/suspicious, one was an electrical failure, and the last was unknown.

In addition, 27 of the 31 fire reports describe fires that were extinguished using one standpipe line (see Appendix G). Twenty of these fires occurred in WTC 1 and the other seven occurred in WTC 2. A majority of these fires (19) are labeled as incendiary/suspicious or unknown, while the other causes of the fires are attributed to short circuits, abandoned material/cigarette, welding close to combustibles, and a mechanical failure. The dates of occurrence for these fires range from 1973-1999, with a majority (23) occurring between the years of 1973-1985. These fire incidents did not result in any casualties, but five civilians and one uniformed officer were injured.

Two of the 27 fires involved a 300-person (April 19, 1980) and a 1,500-person (April 17, 1981) evacuation. These will be described in further detail. On April 19, 1980, at 2:06 p.m., the FDNY received reports of an activated smoke detector in the return air duct on floor 106 of WTC 1. The FDNY also received reports of heavy smoke on floor 106, light smoke on floor 109, and heavy odor of smoke in stairways A and B. The report notes that while only one standpipe was used, approximatcly 300 people wcre evacuated from the Windows on the World restaurant on floor 107 via stairway $\mathrm{C}$ (which was clear of smoke). The fire cause was labeled as abandoned or discarded material and involved plastic material. This fire did not cause any injuries or casualties.

On April 17, 1981, at 9:18 a.m.; the FDNY was informed of a fire on floor 7 and a smoke condition on floors 7 through 11 of WTC 1 . The FDNY hooked up one standpipe and extinguished the fire located in an air conditioning unit in the "MER" room on floor 7. The cause of this fire was labeled as a mechanical failure. The fire report notes that the Port Authority personnel reported an evacuation of approximately 1,500 people from floors 9 through 23 . However, no injuries or casualties were reported from this fire. 


\section{$2.4 \quad$ FIRE INCIDENTS OCCURRING IN WTC 7}

Table 2-3 presents the significant fire occurring in WTC 7. There was one significant fire found for WTC 7, and the fire report is included in Appendix H. Table 2-3 provides a summary of the fire incident information from FDNY records, which is followed by an individual paragraph on the incident.

Table 2-3. Significant fires in WTC 7 extinguished by sprinklers and/or multiple standpipe lines.

\begin{tabular}{|c|c|c|c|c|c|c|}
\hline $\begin{array}{c}\text { Significant } \\
\text { Fire }\end{array}$ & $\begin{array}{c}\text { Incident } \\
\text { Date }\end{array}$ & \multicolumn{1}{|c|}{ Fire Location } & $\begin{array}{c}\text { Number of } \\
\text { Sprinklers } \\
\text { Activated }\end{array}$ & $\begin{array}{c}\text { Number of } \\
\text { Standpipes } \\
\text { Used }\end{array}$ & Cause of Fire & $\begin{array}{c}\text { Material } \\
\text { Ignited }\end{array}$ \\
\hline 1 & $5 / 20 / 88$ & $\begin{array}{l}\text { Construction shanties } \\
\text { on floor 3 }\end{array}$ & $\begin{array}{l}\text { Multiple, } \\
\text { number not } \\
\text { listed }\end{array}$ & 1 & Suspicious & Shanties \\
\hline
\end{tabular}

$\underline{\text { Significant Fire \#1 }}$

In WTC 7, a fire occurred on May 20,1988, at 12:38 a.m., in the construction shanties on floor 3. Although the fire report does not specifically note the number of sprinklers that activated, the operations notes state that Ladder Truck 10 found the sprinklers (noting more than one) in operation and shut them down. The FDNY had to complete the extinguishment by stretching a line from the standpipe to the fire source. This fire is noted by the report as being suspicious in nature and the flame damage was confined to the area of origin.

It is possible that the fire incidents that were not spccifically highlighted, especially those in the areas without sprinklcrs, involved other methods of extinguishment before FDNY arrival, such as a WTC houseline (pre-connected standpipe hose), hand extinguisher, or bucket of water, as noted on some of the FDNY reports. All other fires, the majority, included in other categories were either self-extinguished, extinguished prior to FDNY arrival (by staff, etc.), or a hand extinguisher was used by the FDNY.

\subsection{SUMMARY}

In summary, 16 significant fires occurred in WTC 1, 2, and 7, with 12 occurring in WTC 1, three in WTC 2, and one in WTC 7. In addition to these, 31 fires occurred in WTC 1 and WTC 2, which involved the use of one standpipe (with or without the activation of one sprinkler). Of these additional 31 fires, 23 occurred in WTC 1 and eight occurred in WTC 2. The following paragraphs will summarize findings from the 16 significant fires that occurred in all three buildings.

After reviewing the 16 significant fires, trends developed relating to the time of day that the fires occurred. Overall, 12 of the 16 fires occurred between the hours of 6 p.m. and 4 a.m. The fires that occurred during office hours (between 7 a.m. and 6 p.m.) included a dumpster fire in the floor 43 elevator lobby (WTC 1), a dumpster fire on floor 106 (WTC 1), a kitchen fire on floor 107 (WTC 2), and a bearing overheating in the fan motor room on floor 108 (WTC 2). Almost all of the incendiary (arson) and suspicious fires ( 5 out of 6 fires) and unclassified or unlisted fires ( 4 out of 5 fires) occurred after business hours (before 7 a.m. and after 6 p.m.). 
In addition to the time of day of the fire, trends in the cause of the fire and the materials involved in the fire can be highlighted. Of the 16 fires and their causes, five were labeled as unlisted or unclassified, six as suspicious or incendiary, two as discarded material, and three as an electrical failure or mechanical failure. For the material involved in the fire, eight reports noted trash, waste, and supplies; two reported not listed or not classified; one reported furniture; three reported electrical equipment; one reported duct work; and one reported shanties were the material involved in the fire.

Lastly, the location of the fires throughout the buildings was of interest. Of the 16 fires, 4 fires were concentrated above floor 100 and 6 fires were located in the basement. The others (six fires) were spread throughout the rest of the building.

\subsection{ATTACHMENTS TO THIS FIRE HISTORY}

Appendixes A through $\mathrm{H}$ are included as supplements to this report. The first three sections, Appendixes A through $\mathrm{C}$, are explanations of the numeric codes used in the fire reports by the FDNY. Appendix A is included to explain the codes for the fire reports produced prior to and including 1980 , Appendix B is included to explain the fire reports produced from 1981 to May 31, 1987, and Appendix C is included to explain the fire reports produced from June 1, 1987, to the present. The report code explanations are divided into the same sections as the fire report and give short descriptions for the numbers used in the fire report under each section. For example, if the ignition factor for a fire occurring in 1990 was given a number code of 54 , the reader can find that the cause of the fire was a "short circuit, ground fault."

Appendixes D through $\mathrm{H}$ are reproductions of the actual fire reports produced by the FDNY on the significant fires highlighted in the sections above. The reader can use Appendixes $\mathrm{A}$ through $\mathrm{C}$ (depending upon the date of the fire) to read the fire reports in more detail than what is provided in this fire history report.

\subsection{CONCLUSIONS}

From the information contained in FDNY fire reports and fire investigation records provided to NIST, 47 fires occurred in WTC 1, 2, and 7 that were of sufficient size and duration to activate multiple sprinklers or were estimated by NIST to be capable of doing so, over the time period the buildings were occupied. This total does not include the major 1975 office fire in WTC 1 or the 1993 bombing.

The records indicate that in areas protected by automatic sprinklers, no fire activated more than three sprinklers. Three sprinklers would provide coverage for a floor area of approximately $675 \mathrm{ft}^{2}$ $\left(63 \mathrm{~m}^{2}\right)$. This area is much smaller than the $9,000 \mathrm{ft}^{2}\left(800 \mathrm{~m}^{2}\right)$ damaged by the 1975 fire in an office space unprotected with automatic sprinklers.

Many of the fires that occurred were recorded as suspicious or unknown in cause, occurred during offpeak work hours, and involved materials such as trash or paper-based supplies. In cases where sprinklers were activated, the FDNY records indicated that the sprinklers either extinguished the fire completely or aided in controlling the spread. 
This page intentionally left blank. 


\section{Appendix A \\ EXPLANATION OF NUMERIC CODES PRIOR TO 1980}

Explanation of Numeric Codes Used on Fire and Emergency Reports -

Prior to 1980 


\section{CLASSIFICATIOW OP BUILDING gY USE}

COMUERTAN

Bank

Brevery

tock

Department 5 toro

Electric Power Plant

pactory;Hulti orce-

pancy

actory: single

orcupanicy

Foundry

Garage: Non-5torage

Gas Works

Lumber $=$ ard

Hotor Vehicle Pepair

Shop

OfFice butlding

oil selling station

oi: storage Plant

Pier, Mharve, Dock.

Bulthear Bulleing

Restaurant. Diner

shed. Newsstand,

shanty

shipyard, Drydoek

Stable

steas cenerating

plant

stort Builaing.

Jaxpayer
warehouge, slore

hot:se

store boilding

Private oweling

pugr.ic

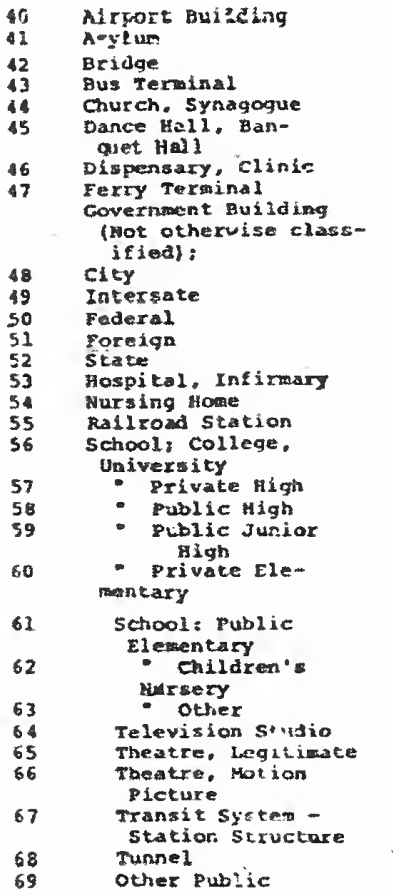

\section{RESTOENFIAL}

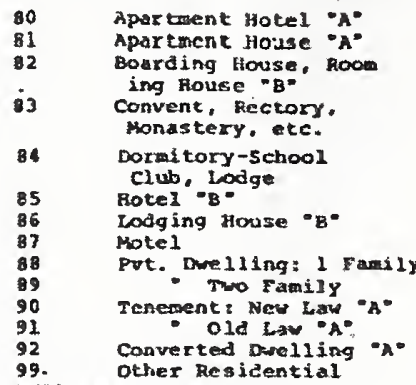

9. Other Resicential

\section{BJILOTWG STATUS}

1 occupled

2 Partiy occupied.

3 Partiy Occpuied, De-

teriorating

Under Dewolition DAMGE (to-tiuilding or Contents)

1 Lone-No Appreciable

Medius 16:-49:

leavy 50 \& Greater

AREA PIAE ORGIN - FLOOR.

or outelde bal zaling

to lat rook

94 gith and wigher

95 attic

proof

Raserenter

collax

sab-celiax

FREA FIRE ORG:N-ROOM OR -WREA

Area rot in building

Attic

himing

Balcony

Bathroos Toilet

Bedroow. Sleming

Area.

Celiling

Cellar

Chimer

Classroom Lecture

Area

crosert

Court-Exterior

Court-Inte

Dining Roow, Dining

Area

Duct-Air Conaition-

ing

Duct-Exhaust

Plooring

Furnace Poon

Hal lway-Private

Hallway-Public

Incinerator Cl set

or, Reor

Ritchen, Cooking
34 Living Roow

35 Lobby

37 Machinery Poon

38 Olfice Area

Operating iaboratory

Area

Partition

Porch

Projection bootr

Recreation Area

Poo

Sales showroom Dis -

play Area

Sha:t-Duct, Pipe

Shas $t$-Dumbwa it

Shaft-Exteriof ight

shaft-interior Light

Shaft-vent

shipping

shipping Receiving

no hrea

stage

storage

Storage Room Area

acant-ROOm, Apart-

ment or area

Work Area workrocm

ther Areas. Not

Classified (state

$$
\text { area) }
$$

AREA PIRE ORICIN-OCCUPANCX CASSIFICATIOD

COMPE RCIAL

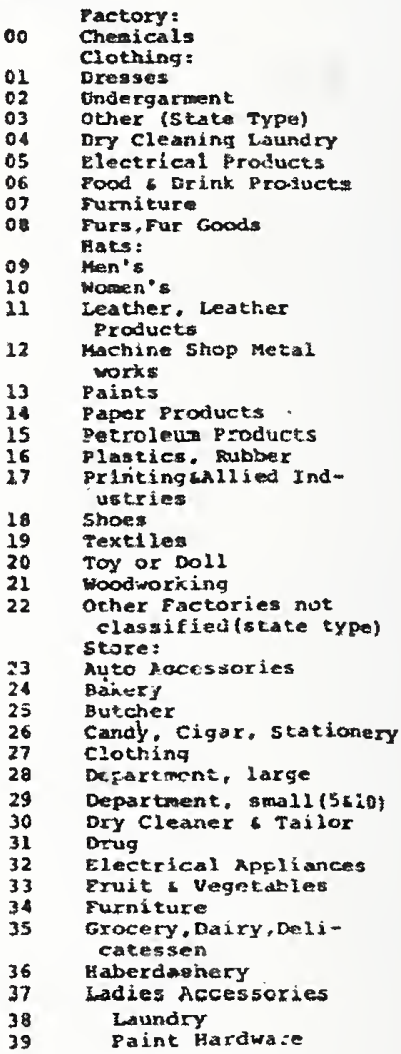

(2) 
Restaruant wncheonette

Shoe

Shoe Repair

super Market

Tavern

Other stores no:

classifiedistate

(Tpe)

Garages :

Non Storage

Storage

oil selling siation

Motor Vehiele Re-

pair Shop

office Builaisg

warehouse:

File

Paper, Rays, Fibre

Other (state type)

Frefght Depot

Pier

shipyard

Luaber Yard

shed, Newstaod, Shanty, etc

other Commericial

Building oceupancies,

not classified (state type)

\section{RES IDENTIAL}

60 Apartinent botel,

Hultiple Durling"A

61 Apartment Roase, Mul-

Apartiont hoase:

62 Boording House, Roowing

House multiple

arelling to.

63 Botel, Multiple
Dreling B
64 Lodging gotse, $151-$

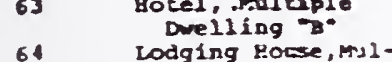

64 Lodging gonse, Msl-

65 2rivate Durling

66 Rectory,Corment.

Monastery

Tenement House.

ver Law, malti-

ole Durellin "A"

68 Tenemont Pouse, Old

Lav, mitipie

69 Cther Residestial

not classified

(state type)

PUBLIC

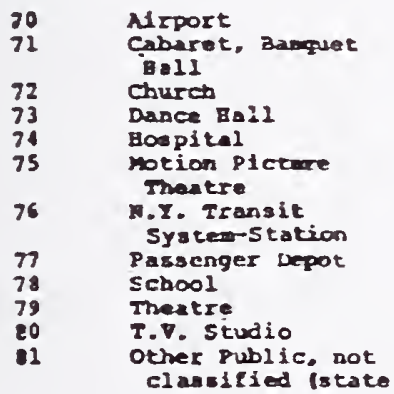

clansified istate type)

\section{MUNER EXTENSION}

\begin{tabular}{|c|c|}
\hline 00 & $\begin{array}{l}\text { Conflned to area } \\
\text { of origin }\end{array}$ \\
\hline cl & Cock loft \\
\hline 02 & $\begin{array}{l}\text { Door or opeaing } \\
\text { between roams }\end{array}$ \\
\hline $\begin{array}{l}03 \\
91\end{array}$ & $\begin{array}{l}\text { Ploor } \\
\text { Ball Stainugy }\end{array}$ \\
\hline os & Paz:ition \\
\hline $\begin{array}{l}05 \\
07\end{array}$ & P1pe Recess \\
\hline $\begin{array}{l}07 \\
08\end{array}$ & $\begin{array}{l}\text { Shalt-Dumbuaite } \\
\text { Shaft-Elevator }\end{array}$ \\
\hline
\end{tabular}

09 Shaft-Air,light.

Chute, Duet, etc.

10 Celling

11 Hindor

other (state houl.

CLASSIFICATION BY TYPE FIRE OR EMERGENCY

\section{TRANSPORTATION PI ZES}

\section{Shif, vessel \\ 9 Moror Vehicle \\ 89 Other Iransportation \\ (state type).}

\section{HON-STRUCTURAL IFES}

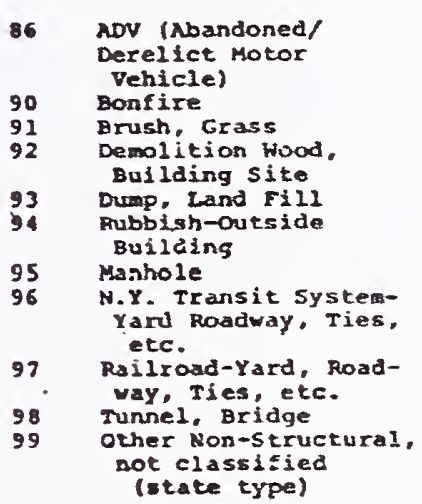

\section{EMLRGENCY}

02 Chimey

03 Elevator. Escalator

04 Explosives Escort

o5 Pirst Aid - Assist

Person ( $\mathrm{s}$ )

06 Firat Ald - Resucita-

tion

Marine

Procarious Condition

5igns. Trees, etc.

Subway-Railroad

Water Leak

Bamb-Unexploded,

Scare

12 Collapse-Cave in

13 Collision-Vehicular

Incident

controlled Fire.

Permitted

Flood Condition-

Broken Water Main

Inclnerator

Ieak-Fuel oil, Gasóline

etc.

Leak-I1lus, Gas, Flam, vapor

Lightening

Q11 Burng

Person Locked in.

locked out

Power - Eiectrical

Presaure Rupture

Refrigerant reak

smoke condition.

odor, Fures

Sprinkler

steam Discharge

Other 
This page intentionally left blank. 


\section{Appendix B \\ EXPLANATION OF NUMERIC CODES FROM 1981 TO 1987}

Explanation of Numeric Codes Used on Fire and Emergency Reports -

From 1981 to May 31, 1987 
Thpen of sapart

1 sivertar ad.

2 Trasseartohion Fü.

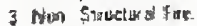

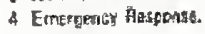

5 Folse diber.

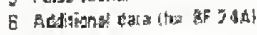

that hapartiod

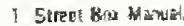

3 Ttephase

3 Heros.

4 Class J-MEnar. PFR

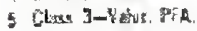

E Clum 3-Dines Aurmtatic PF

3 Cless 3-ERS

a Siret $80 \mathrm{Fa}$

9 Class 3-Manjor. IORY

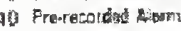

\section{Intrist aberto}

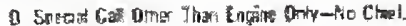

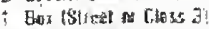

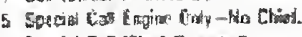

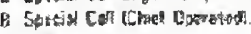

a 5 แ

Hith

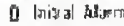

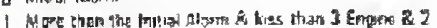

Laeds Ca. at wort

2 rat diarn.

3 3nd Marm

4 44: Alaspron

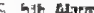

E. Simbitanum

5ignna: 7.5 .

Hove Extangyiation

a Ro pore Aatroal

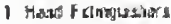

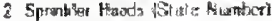

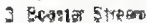

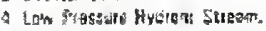

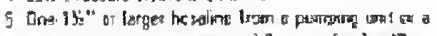

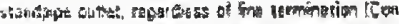

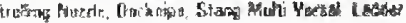

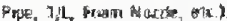

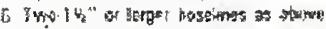

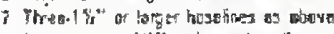

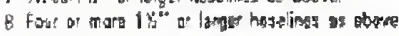

iy Olher IState How?

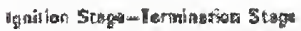

3 Sirtolde Stage, be tart any flome.

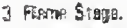

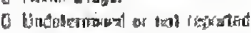

Eอบง

Anationg Eysterse

11 cencers heating uxat.

12 Feter beetzs

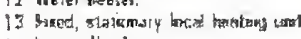

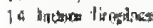

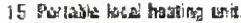

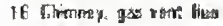

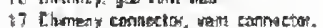

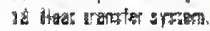

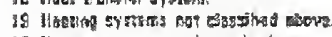

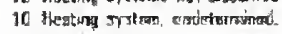

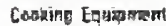

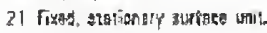

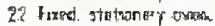

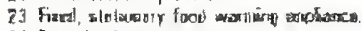

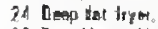

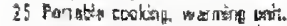

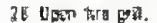

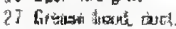

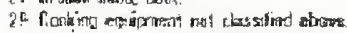

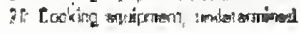

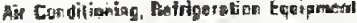

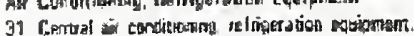

32. Walet cooling deriér. nwot

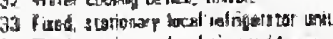

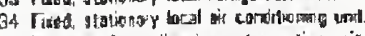

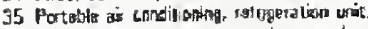

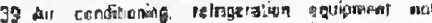
shestion sove

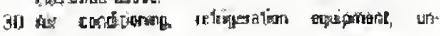
tolurninad.

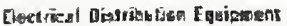

41 Fined wiratag.

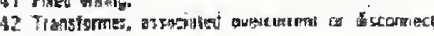
equiprient.

4. Heter, meles twin

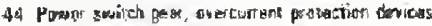

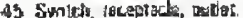

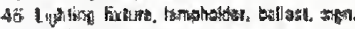

4) Corta plost

4. I ma, that bub.

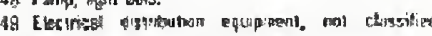
abure.

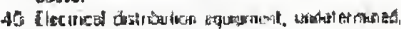

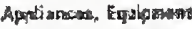

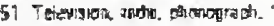

52 Dryer

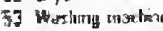

29.

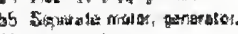

Scis Hand tack

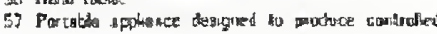

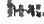

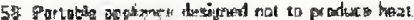

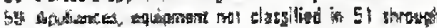
58 .

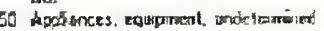

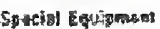

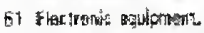

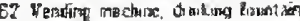

63 atfate mustine.

E4 gimed equpment dyence.

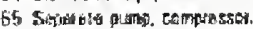

E6 Fombutwor mane.

6) Gonte pra

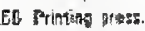

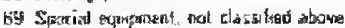

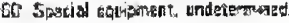

Procsesion squipmont

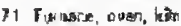

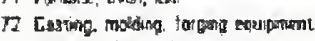

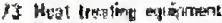

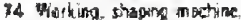

15. Coyth medhas

76. Panting equiponen?

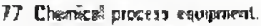

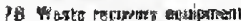

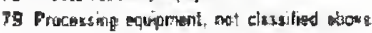

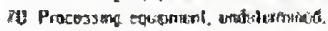

Serwiea, Haistananes Equigenen1

O1 hicines atx.

प्र2 Berinzy. luake.

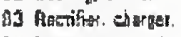

84 Texpot, 证 thet

85 Are. Iamp

46 Elerass.

B) Torchas.

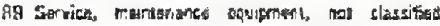
aboum.

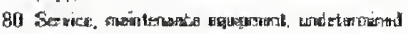

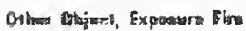

S) Bo;

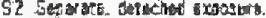

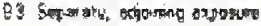

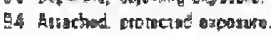

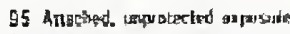

Jo Vehrek.

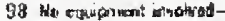

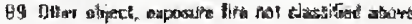

SO Ither mitert; axposure tire.

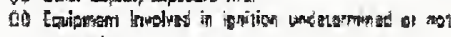
roparad.

FDRE OF HEST DF IG

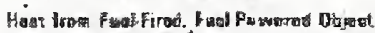

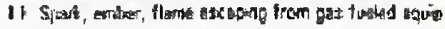
mant

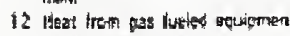

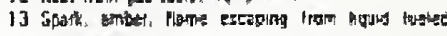
tquprasens.

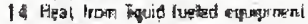

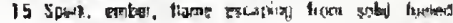

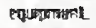

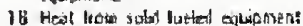

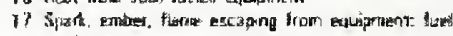
nas known.

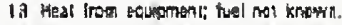

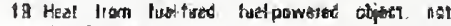
dassifrac sovove.

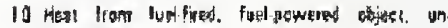
Baszmined.

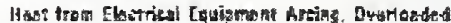

21 What basmet shon tattali sic.

2. Strul circut' aec from mothancal da mage

11 Shon circurt ace from osfecrivo, wom ingulawon

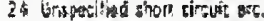

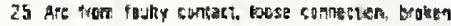
conductor

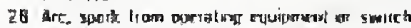

3) Heat from inerikatad equigiment

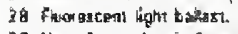

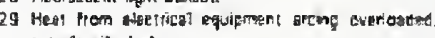
nat chassilied stove.

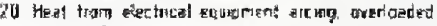
undueimined

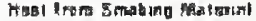

31 Cugsrate.

32 Enge

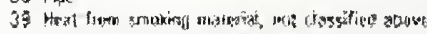

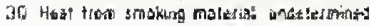

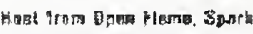

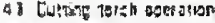

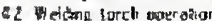

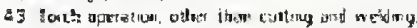

at Caulle laper

45 bisicik

40 igleter.

$4 ;$ Open fire

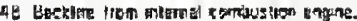

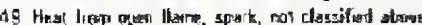

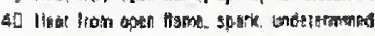

Heat fram Hot Dbject

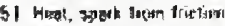

52 Hotitun, ho? matrial

by Hot sutser. assh

G4 Elscusc tamp.

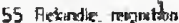

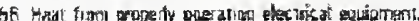

5) Haor lion improperhy oparatog electic sl everponent.

59 Heat fiom tand obyext. ant classifind abouve

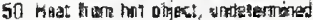

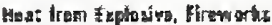

fi) Eaphasinge

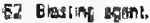

63 Frembits

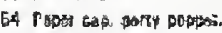

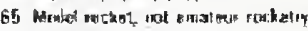

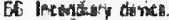

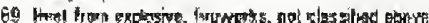

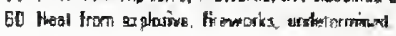




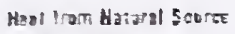

17 swis red?

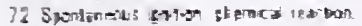

Tis liaring dischuly

is Stric dis andegt

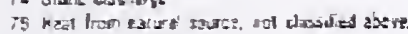

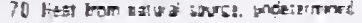

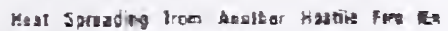
pesury?

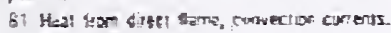

62 Rruight heal

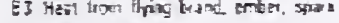

at Foeducted bras

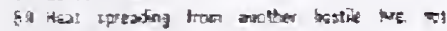
fissilix abere

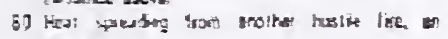
sะ1e-ane.

Dither foum of fian ef ignertions

8? Wdiste tor or ol heal of tortion

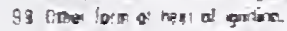

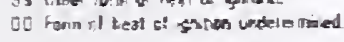

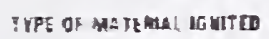

Ges

if hase of the

12 LP ect ças II' ase di mo

12 Mentwivec gas

19 :

12 inestetiug ga:

$: \bar{E}$ sतe

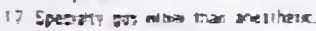

is Co ret ciess wed dors.

is Cas.

flemabie. Candustion Ix aid

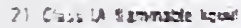

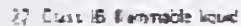

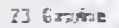

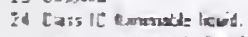

25 Cxs3 4 cankentrie ficud

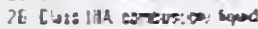

3 Lass lite sonors! de hed

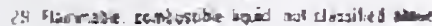

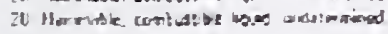

Velative 5050 Chente

$\because 1$ in: fease loci:

3. forese inglood.

51 idat.

3t hemerve. Tex Is

75 AOEl is GML, rems

25. Comiest by ne

31 sold enamas lapasty trol

If Redental mites

39 run'te stio Jisrky agt daciled noove

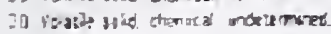

postic

il polpaplt ane

\&. Potistige

2) Dolvungl

it Pationik

is Pelizerv.

is todresers

fo Mastis. m: casplet orme

40 Pagr: valoipritex

sou. Prosuar

It puleer.

Sit inik

S? icurter

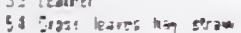

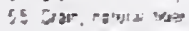

it. [eal crir bestete ped

S? how =ret

S\& Tabers

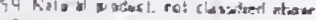

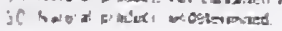

Wesd, Docer

6! Crewry woot

is leled bul terswen toon

83 Süse wot.

be Hrout stavings.

85 Karbucer, plyweod.

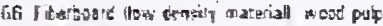

5) Paper, miested. unconted

68 Carluan

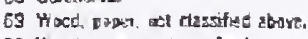

fo these papex, antelemsind

fabric Teritis, Fur

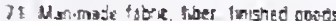

iz Coltge, fapon, concon labric frished quect

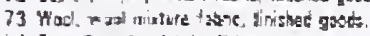

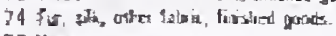

$75 \mathrm{Wh}$

36 Hinsa kav

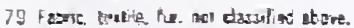

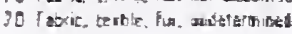

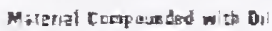

BI Li: $=194$

B? Ci ch.t

8J Irezted asulu coavd Bapm

6: Watertiona have

ES Dip iags

86 Asshah buaret marenal

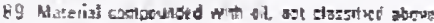

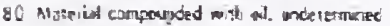

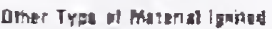

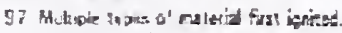

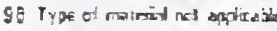

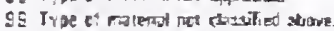

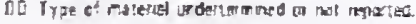

PURM OF MATEARLICNITE

Strutatel Comporsh Frogh

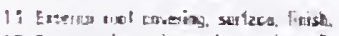

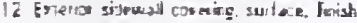

13 Ea?

14 Flocir cowesno sultere

is Intertar wall cotiong gulase ltens permeamily "Hued to $n$ al and ear sulast.

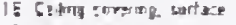

17 Sinteive membel, Hatrong

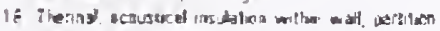

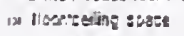

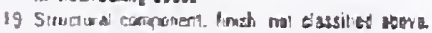

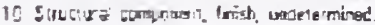

Fupt?

21 vorowsure sofa, unis velicle sezis

72 Nars pretsteres inaw. tomet.

$7:$ Exingloy.

24 liming blart.

28. applence hasimy w sasin

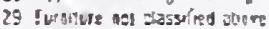

20 fuinture uduturnos.

3ols Gouds. Wesring Appare!

31 Wa:liess 2ar. a

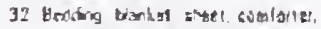

33 lime wher the wald on

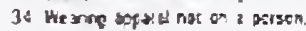

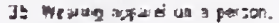

3t Cunga terd dipondr tadislir

37 lixeds nol nid: $a$

38 ke powp

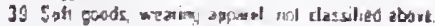

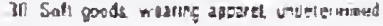

cerament metostionel Me inriel

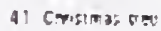

43 Jecosion lor agerial rom

4) 3रet

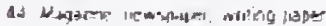

45. To:, namse

46 Awning

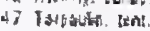

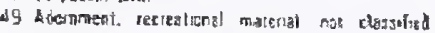
ebret:

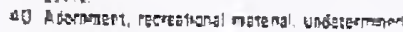

Supliorn, Stases

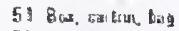

52 Bosiet, barrel.

53 thiles, skid ane is uou

54 Rimen cood, nuipe, yam

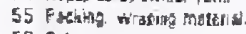

56 Eak simase

57 E. sioras.

38 C C

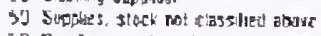

30 Suppers. Itack. unotersmand

Fow Transior Equipment. Fuel

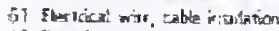

al Trasstormz

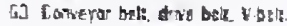

G4 Titk.

55 funi.

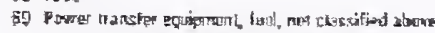

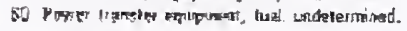

Geased Form

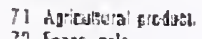

72 feace. pate.

73 Fertilizer-

is kibaring, twerng forra

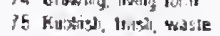

76 rasing mal ariats

$375 \mathrm{~s}$

Syakiod forre

81 Juse fider brat

82 protichicr, isplasnes

8.3. Alamize: yaprized los

8. Erats.

E5. Paleticec materisl. material stored an pe

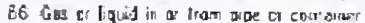

ET qoget naterial.

ge Athosive.

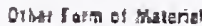

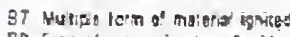

95 forn al marentil not sopicable

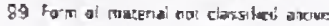

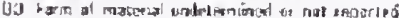

ICYITEOF FACTOR

Insendiary

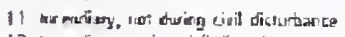

12 uncenciaty, cuing civil tosibaxt

Suspixions

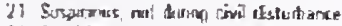

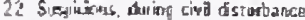

Miasse of Mest of Igraten

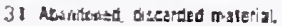

3? Thaning:

3.3 sting asleep

34 in aseanate esncid of wer lue.

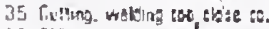

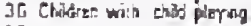

3) urcongriout. mente, phpsical mopaement

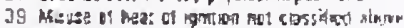

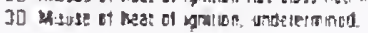

Mituat wat Marial lgaine

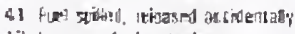

42 linomer lixhog tectuknues.

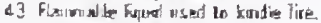

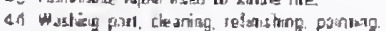

15.) Irpisos contaras.

Sf Combustible laso those if MEII.

d7 inpoper sorage

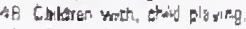

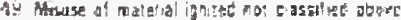

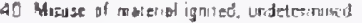


Methonical Foihure, Mallanction

51 Porl talure teak, break.

52 Automatic contual fallurs.

53 Meous control lalure.

54 Short creztit. Ground ladt.

55 Other dectrical Iavie.

56 lack of mainenance. worn ant

57 Bockfire.

59 Mechanic da filure, mattunction not classified abor

50 Mecrinical fature, mallunction, undetermined.

Design, Constrotion, lastollation Defieioncy

61 Design deficuency.

62 Construction teficiency.

63 fastalled too close 10 combustibles

64 othe instalation ficiency.

65 Property too clase to.

69 Dasign, construction. installation deficiency not claseified chave

60 Design, construction, instatiation deficiency, undere mened

Opertional Deficiancy

71 Colision wertuzn knockdown.

72 Accident ally turned on not turned off.

73 Unatiended

74 Dreikaded.

75 spomaneous hastung

76 impraper starup. shutdaw peocedures

79 Opertitonat diciency not classified above.

70 Operationd deficteracy. undetermed

Matural Conditiea

61 Nugh want

82 Ear thequak

83 High water, nachuding thonds

84 Lightrang

B9 Nanual condition not classifiod above.

80 Natua al condtion, undetermined.

Othat Igaition foctor

91 Artanal

92 Apknded trom a payious the

99 Other igntion tactor not classfived above.

00 tormion latian undetermmed of not raported.

Censtruction Class

C No Buildong Involved

1 Tieproof Siructure

2 Fae Protected Siructure

3 Non fireproof Structure

4 wood frome Structure.

5 Metal Structure.

6 Heary Twoter Structure.

Clossificution of Building $B Y U_{n e-C w m a r c i a l}$

0) Bank.

02 Brewery.

03 Cos Pocker.

Q4 Deoartment Store

05 Electnea Powe Piom.

06 Factory. Muli Decupane

of tactor. Sungle Oetuaney.

08 Foundiry.

09 Freigh Depo:

10 Garage: Mon Sterage.

11 Garage: Sior ape

12 Gas Works.

13 Lumber Yard.

is Hor Vehicle Aegai Shop.

15 Dffice Buidng

16 De Seting Siarioi

17 O) Slorage Piant

18 Pier. Wharve, Dock, Gulkhead Euidiog

19 Pestau ant, dner.

20 Shed. Newsatant, Shanty

21 Shovad. Drveloct

22 Stabie.

23 Stean Generating Plamt.

24 Siove Buidong. Toxpayer.

25 Wathouse Stcretiouse.

26 Siare butiting 8 Prwate foweting

39 Othe Comperal.
Cissulfitatien of Bulloing By Uea-Poblis

40 Airport Building

4) Asylum.

42 Bridas.

43 Bus Terminal.

44 Churct, Symapogue.

45 Dance Hall, Benguet hall

46 Orgpensary. Cline

4) Ferv Terminat.

Gopernment Buldungs-(Not otherwise claswifed):

48 City.

49 kiterstate.

50 Feder al

51 for won.

52 Sinte

53 Hospita. Infomary.

54 Nursing Home.

55 Hakisad Station

58 Sehoot Collent. Utwwersiy

57 Schoct. Private Itigh

55 Sthoot: Pubtic High.

59 Schoot: Pubtic Jr. High,

60 Schood Private Elementary.

6) Schoot Puthe Elementary.

62 Schood. Chithen's Musery.

63 Schead: Other.

64 Television Sivotio

65 Thesure. Legitinute.

66 Theatre. Mọtion Picture.

6) Ilaash System-Station Structure.

68 Trand.

69 Othe Public.

Aexidoatial

90 Apatrien! Hoid "A "

B) Apartment House "A."

82 Gouding House, Foomng House "B "

83 Convent, Riectary. Monastary, He.

B4 Bormitar-School, Chat. Letge.

B5 Hatet "B."

B6 Lodging Hause "B."

B7 Matel.

88 Pruate Dwelling: One fambly.

gg Pivare Owellong: Iwo faniyy.

90 Tenentent: New law "A"

G) Tenemert: Old taw "A"

9'. Converted Dweting "A."

gg Othe Aesidential.

Buituin States

1 Getuped.

2 Partly Occugied, Good Condition.

3 Partly Oceuped, Deternos ating.

4 Vecant.

5 Under Derrotition.

6 Under Construction

Domape no 8uiding or Contentul

D Siona.

$111015 \%$

216 ?0 49\%

$350 \%$ or Greater.

Arae tire Origia-float

00 Dutside Butcion

01 is a floor.

ta

94 94d: and Hosher.

95 Artic.

96 Rioot.

97 Basement.

96 Colliat.

99 Silberitar.

Are Firs Origin-Hoom of Area

10 area Nơ in Buading

11 Antic.

12 Awning

13 Baicony.

14 Basenent.
15 Bathoom Toilet.

16 Bedroom. Steeping Ares.

17 Celing

18 Cestar.

18 Chimeny.

20 Classioom Letruas Aita

21 Closet.

22 Cociloht

23 Court-Exteriot."

24 Court-interior.

25 Dining Hoom, Oning Ares

26 Duct-Air Conditioning.

27 Oart-Exhoss?.

28 Floor the

29 Furase Room

30 Hallway - Private

31 Hathway-Puble.

32 incenerator Closet or Room

33 Kitchen, Cooking Area.

34 Linng Axom.

35 Lobby.

36 Machinery floom

37 Dffice Ares.

38 Operating Labornlory Arta

39 Partition.

40 Porch.

41 Projection Booth

42 Recreation Area.

43 Plool.

44 Sales Showrtoons Onsolay Area.

45 Shat-Duct, Pipe.

46 Shofl-Oumbwates.

47 Shath-Elevator.

48 Shats-Exterios Iinga

49 Shat-lateriot light

50 Shaft-Vent.

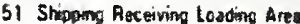

52 stago.

53 Slakway.

54 Storape foom Area

55 Vocast-Rloam, Apartment of Aree.

56 Work Area, Workicom.

57 Other Aruas, Not Classitoed tstete area

Ares Fir Origin-Desupancy Clascification-

Commereiol

Foctery:

99 Chencals.

Clotbing:

Di Oresses.

02 Unsergament

D3 Other istase typet.

04 Dry Clearing Laundry.

O5 Electrical Products

of Food 8 Drink Prodtets

D7 Furriture.

OB Furs. Fui Goots.

Hats:

09 Mer's.

10 Wemen's.

11 Leather, Leather Products.

12 Machine Shop Metal Worts.

13 Pants.

14 aper Products.

15 Petrolaum Procucts.

16 Pastics, Rublus.

17 Printing \& Aloved Industries.

18 Shaes.

19 Textiles

20 Tay or Doti

21 woodworking.

22 Oth factaries Not Classifiad istate typet Store.

23 Auto Accessoties.

24 Bathery.

25 Butchet 
Arst Firs Origin-Decapancy Classifisationicontmuedi

Stere:

26 Canty. Cons, Stationery

27 Clotive.

28 Cepariment lage.

29 Oepartment, Smal 158101

30 Dry Ceares $872 x$

31 trus

32 Electroci Apolines

33 fivi 8 Veget ables.

34 furmtixe

35 Grocky. Odry, Osicatessen.

36 Haberdasiery

3) iacies Accessoriex.

38 iandy.

39 P an Hattwere

40 Restar ant Luncheonetie.

41 Shot.

42 Sine Rem

43 Saper Martet.

44 Tarth.

45 Othe Stores hor Classilied istare trpel.

Coreges.

46 hon Starage

4) Siarue.

18 Cor Seleng Stain.

49 Moto Viunct Resw Shoo

50 other buding

$$
\text { worativese. }
$$

51 fite.

52 Pacer. Aags Fibre

53 Other Istale inpes.

54 Frengh Deoor.

55 Ries

56 singerd

57 lumber You

58 shed. Newssiand. Shanty. alc

59 Other Commerod Budong arcusances. Not Classufect lsiate inder:

Aresulestid

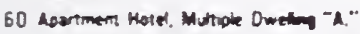

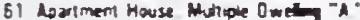

52 Bozrding House. Roonng House Whote Oweting $B$.

63 Hote. Wultele Dwetng 8 .

64 lodping House Munnile Owehn 7 ."

65 Prvare Durting

65 sectory. Coryent, Honesiery, ets

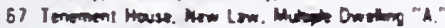

65 Tenemen! House, Ot $\mathrm{Lm}$. Muhite Dwthe "A."

69 Ome Residental, wa Classifed bere rop

69 Oner

70 Arper.

i1 Coboren, Bampue he

12 Chureh.

73 Oance $\mathrm{Hal}$

14 Horen:

is Motron Piclue Thealte

76 MY Transit System-Siation

$7)$ Passenger Depot.
78 School

79 ineatre

80 I.V. Studio.

81 Other Puble, Not Classified istute trpel.

Manser Extensios

00 Confined to area of arigin.

01 Coskbth.

02 Oow or Dosswing Between Rootss.

03 Floo:

O4 Hal Starway.

DS Partion.

O6 Ppe Recess.

07 Shyllouratwerter.

O8 Shath-Elevator.

OS Shati-Ax, Iight Chute. Duct. atc

10 Cesing

11 Window.

12 Other fstate now

Number of Deceponsies

011 Derwasty.

0220 Octupmes.

9999 or more Occupancies.

Buildings

(1) du nol spread beyond bulaing of argin.

11 sinicturs o veticle.

99 of more buthings vehictes.

Nore. Form Bf 244 murt be submitted los each buldon or vehcle biod in this coded sooce.

Smoke Detecter

O No detector presem.

I lonzation fype. power fiscomected or banety iemered by occupent.

2 lonzzition trpe, provided eaty warning.

3 lonsation inpe, fated to operate. battery powered

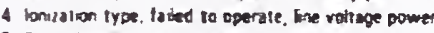

5 Phelotetinc type. powe disconnected of buttery iemoved by occupant.

6 Pholoetectic iype. pounded eath wornm

7 Protoecetik iype. falled to operite, bantery onwered.

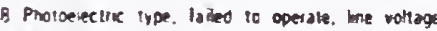

9 Nor possable to determune if detector operaled or no

Clessification by Trpe firs or Emergency

Transportation Fires

87 Sim. Vessed.

88 Mora Vehich

89 Other Transportation Istate tyoel

wen Structural fires

86 AOV labandonediOer ther Molar Vehacle:

90 Bonlue

91 Brush Giss

S2 Demotion wood. Butding Sile

93 Dump L and Fill

84 Rubb:sh-Outude Buildem
95 Mantole.

96 N.Y Transit System-Yad, Roadway, Twes, ote.

97 Radroad-Yard Roadway. Tins, atc.

98 I turnel, Bridge.

99 Other Mon-Structural. Not Classifed istate tyael. Enaergency

02 Chimsey.

03 Elevator, Escumter.

04 Esplosives Escant.

O5 Frst Ad-Assist Persturis!

O6 Fir si Ad-Resosctation.

0) Marine.

08 Precsious Condtion-Sines, Trees, etc.

09 Subway-Paitood

10 wale lask

11 Bunb-Unexploded, Scare.

12 Collepse-Care th.

13 Consion-Vthicular incident.

14 Controlied Foe, Peraytzed.

15 Flood Condtion-Broten Wrat Mon

16 inconerater.

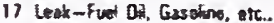

18 lesk-Hum Gas, Fien Vopes.

19 lightiming.

20 or Bumer

21 Person lock the locked Out.

22 Power-fectrical

23 Pressure Rupture

24 Ridriger tent Leat.

25 Smote Condition, Odor. Fumes.

26 Sprinkties.

27 Steam Dseharge

28 Defectrve Alarm Device lother than Sprenktent.

29 Smoke Detector.

30 Other.

Powal tor Equpment

01123 rotis $A . C$.

0224 volts $A . C$.

111.6 rains $D C$

127.12 rotes $0 . C$.

15115 wats 14.

28208 voits $i \mathrm{C}$

$30 \quad 220-230$ valt $A . C$

33231.330 volts A.C.

34331 o hagher vohs AC

502550 rohs A.C.

61 Butane.

62 Coal. Coke. Chartoal. Peat

63 fuel 0: No. 1 or No.

64 toet 0 연 3 or $N$

65 fuet Od. No. 5 or No. 6

66 Gasotne.

67 resesene.

58 (N gas Istord as hquide

69 LP gas istored as fipuid).

70 Natural or illumanating gas los a gas

71 Payer

12 Procane.

99 Oife: 
This page intentionally left blank. 


\section{Appendix C \\ EXPLANATION OF NUMERIC CODES FROM 1987 to PRESENT}

Explanation of Numeric Codes Used on Fire and Emergency Reports -

From June 1, 1987, to present 


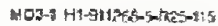

14.1 TYKE OF PEOTI

coce No

1 suclurat

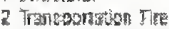

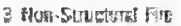

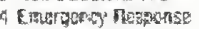

5 Fise Anth

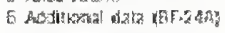

\subsection{HES HEPTETED}

Couc No.

10 it iepions:

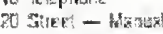

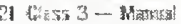

$306 a^{2} 5-8 \pi$

3⿻ Ge

32 Cles 3 Other Astrmatic - PF

aी?

50 Vertial

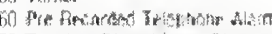

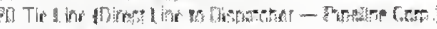

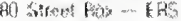

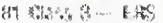

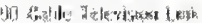

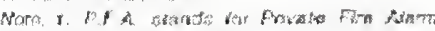

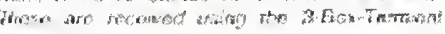

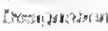

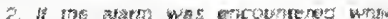

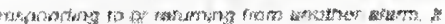

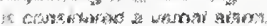

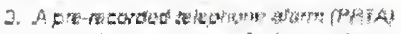

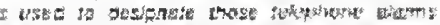

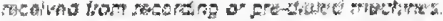

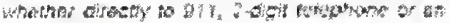
gatum sionters.

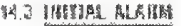

corde Na

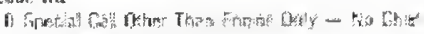

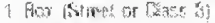

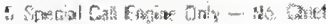

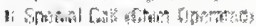

4) 5 क्षे

\section{HA HICHET RLAS}

\section{Live Ne}

(1) in

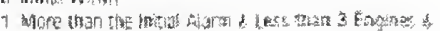
2 Laxder cass. at wark.

2. 20id Marm

is sid Rasm

A sth hivin

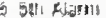

6 Eimutastas:

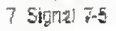

I4 3nsethks

Case He.

I Mandatar

2 Drex:

3 Stater 1 slane

4 Erovibyt

5 onems

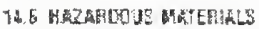

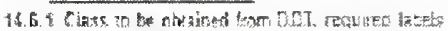

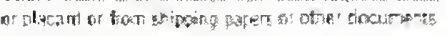
cage No.

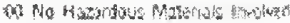

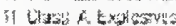

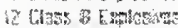

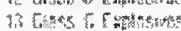

15 etefing Agers

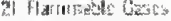

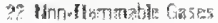

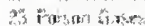

of colve wo

2. Wand
FIRE REGORD CODE LIST

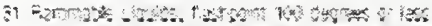

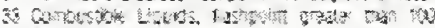
cores

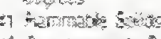

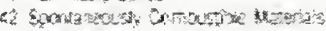

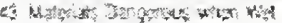

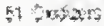

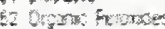

it arest

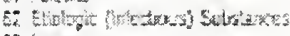

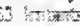

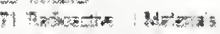

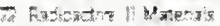

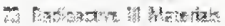

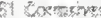

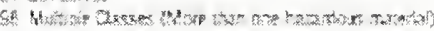

a rater

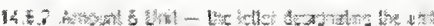

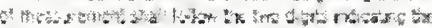

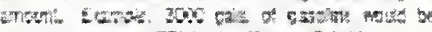

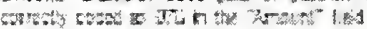

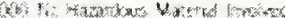

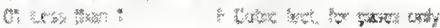

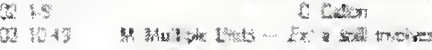

in

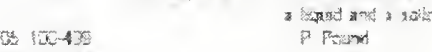

a. $57 x+3$

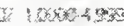

I I

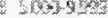

o. $1050+202$

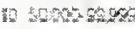

1) $\cos ^{2} 20 \mathrm{~s}$ :

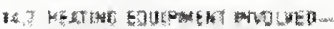
TPE OF FUEL USE

Cxon

i. perasen

2. IE

3. 4 stertret

cost

co

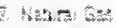

e is 120

Q. Whet

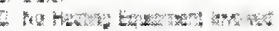

14.8 How ETENGUtSTEO

Conste ha

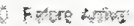

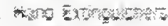

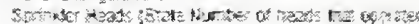

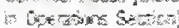

* Exte Strats

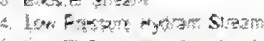

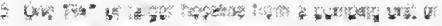

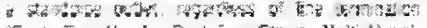

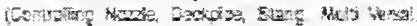

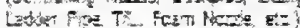

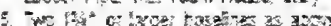

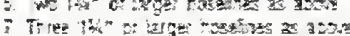

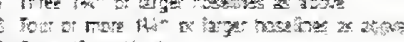

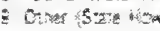

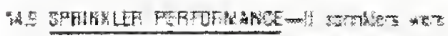

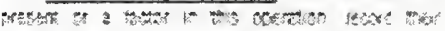
rivand

3.

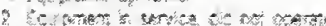

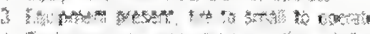

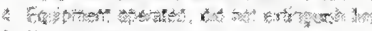

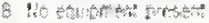

Q Tow

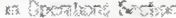

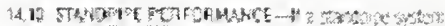

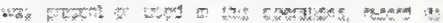

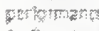

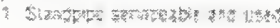

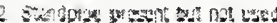

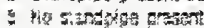

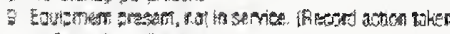

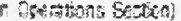

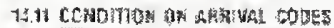

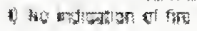

1 Snemeat

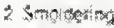

a on the the

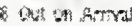

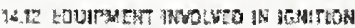

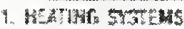

14. sats? partel.

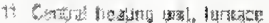

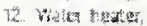

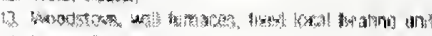

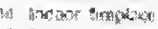

if

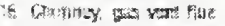

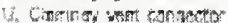

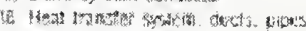

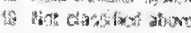

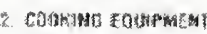

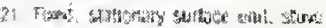

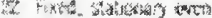

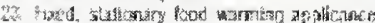

2द tres for ther

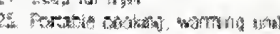

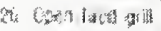

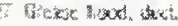

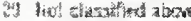

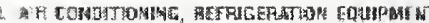

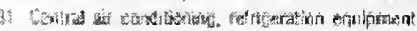

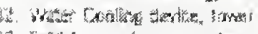

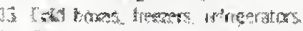

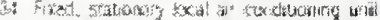

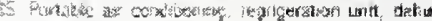
mol 1 tian

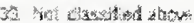

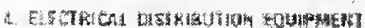

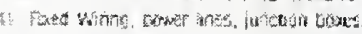

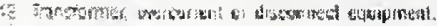

4 M M

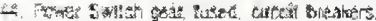

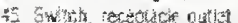

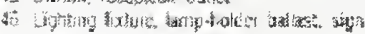

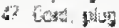

42. Ang kigrt bat

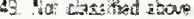

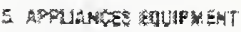

5. 20

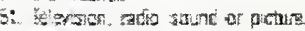

玉2

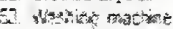

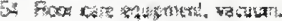

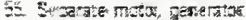

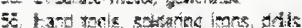

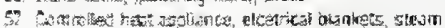

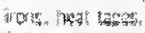

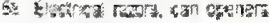

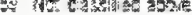

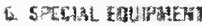

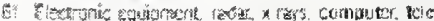

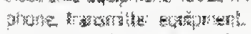

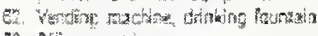

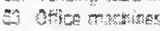

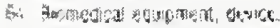

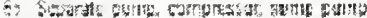

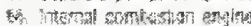

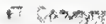

1.4. Prom ores

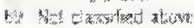


2. PROFESSIKS EOUIPUET

7. Fanece ansi, olr

T. Caviag, matng lornes.

T5. Hadi vetir. 2 grem tenk

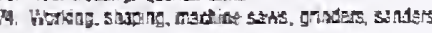

75. Coting machipe aspall soturelug. ritor sperding rotrine

7. Paiding of ter spaning

T. Chemieal pocks resting

7. Wace recosery

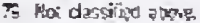

8. SERTEE, MAHERUAC EOUIFET

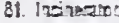

2. Rering. Dro

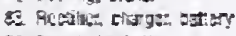

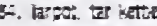

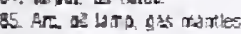

a. Rerato?

8. Vorchas bunsen bernets

se Miol disisind abore

1. THE OUIECTS TYPSURE FRE

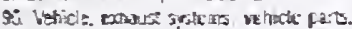

9. No equipment imalued.

31. Other otjea. Lupcsure Fire no classhed abone.

\subsection{PORM QF HEAT ENITHON}

1. HEAT FROW FUSL-FTAEQ, FUE-OOWERED OBJET

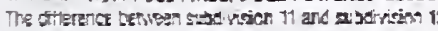

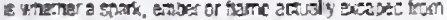

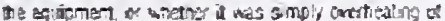

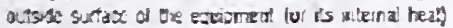

cessing the ipison of narty contesibles.

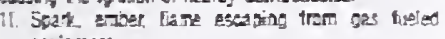
ovineses.

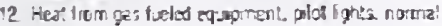
firnez.

35 sak ember fame escaping trot basid tued equipmen:

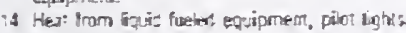

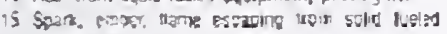
eavic T:Rt.

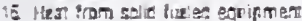

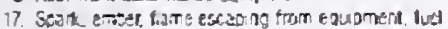
not know.

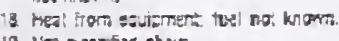

'9 ito cassite show.

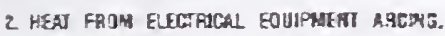
OVERLCLOS

2i. 'Hel cause' short oircuit are.

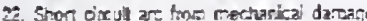

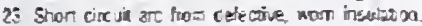

it Urisecitiou som arout ar

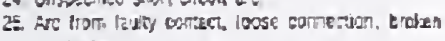
$\cos c=2 x$ ?

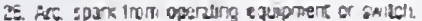

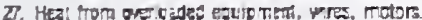

21 fivoresent lints belizet.

2C Nor ciation atore

3. HET FFOM SHOKIMG MATERLAL

31. Cigartle.

32. Cigar
is Pipe

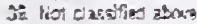

4 HEAT SROM OPEN FLAME SHARX

4! Cuturn torcl apcrilion (s. paraing metals)

42 Weidng iorali operaien forang matalsi.

43 Blan icristes puntes borthes. Bunsen Bumers. soldering fairl stipicing

44 Carde 110

-E Bazch.

4E Ligues (F ane rype)

$\therefore$ i card fas ant

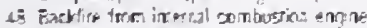

$s$ Ber ciarcilies atrow
5 HEAT FROH HOT OQUECT

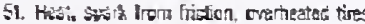

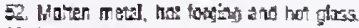

53 He ented ath

54. Elrdic lamp, lagit bulbs

52. Felerindle, reignition

56 Her from propesty opercing slewisal.

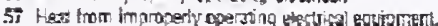

59. Nar dussifięd thane

6. RES FAOM ERLSUES, FAFWORX

61. Exelosives, berras ammuniton

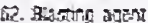

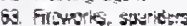

54. Pase: me. pBTy mope

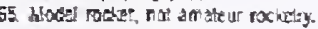

fri the existy dedice

Fi. Not classitred abur.

7. HER FRON MATUFAL SOUACE

7. Sur's heas.

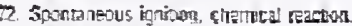

73. Liching G G

7. Suge dsohary.

7. Hol classifez $z a s$

2. HERT SPREADIKG FROS ANDTHER HDSTLE FIRE \{EXPOSURE

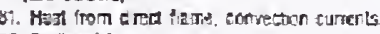

QI. Rasiato thest.

61 Head from flong beando. ender, spari.

84. Coneuzter hed.

89 IN: elesolied abco

Q OTHER FARH OF MEAT OF ICNITHON

97. Kultiple formas of neat of ingun.

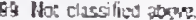

\subsection{TYP OF NATERLLL IONTIEO}

1. Ges

11. Mrom gis.

12. LP. Cry Gas (LP and air mix)

T3 Maglarides sas

14. LP.GSS

15. hwathere gar

Ii Aseryle re

17. Sactialty pas ather than anestheric

12. No. classived abcur

2. FLAMHARIE TUNGUSTIBLE LOUID

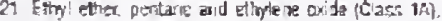

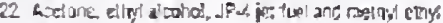
बitme. inlass 18

23 Gasoline

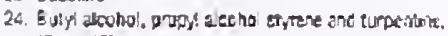
Class 197.

25. Kerosene, tuel Di 1.2 4. 5 and Diesel Fuel

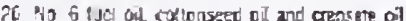
class Ilias.

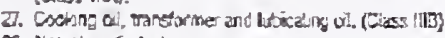

x. Nat clasofited abore

3. VOLTTL IOUC, CHEMICUI

31. Fat, greace (lox)

32. Gnese (nomions). cetiokum palles

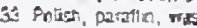

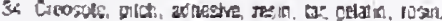
dEe tzall?

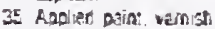

36. Combushble metal mapnesium titanium ano scconum.

37. Sodi: thanical, explostres

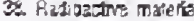

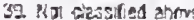

4. PLSTIC

41. กICD PI ATा KS

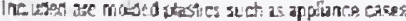

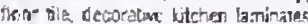

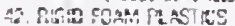

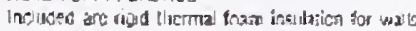
anc ietrigerators.
43. FLEXIBLE PEASITE

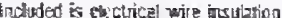

4 GEXIBL FOAMP astics

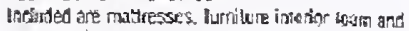
carptit asds.

45. FLH PIATTCS

inctursed are thiyctivene tash begs phoiographic

fian and eogres mals papti.

4) Pastir no: classited above

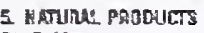

51. Pubber

52. Conic

53. Leather

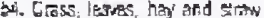

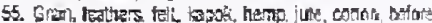
prosussing.

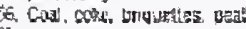

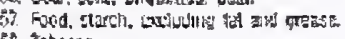

58. thater

50. Na: classified abcol

6. WUUD, PAPER

61. Growing wood. tee

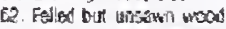

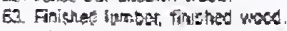

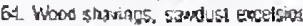

65. Harducard, plimunod.

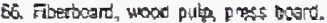

Ex. Paper, untrestad, uncazted

Ex. Cartbard.

69. Nol Aasiled abore.

T. FABRTC, TDTRE, FUA

71. Man-1]

7. Cothor ra, con, conton tabric

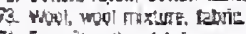

76. Fut sin other baric.

72 ist

T. llomas hi:

12. Rio: dascetien andive.

8. MATERLAL COMPOUNDES WIT aIL

87. Lindeutr.

82 of ciuth.

63. Thesto endor coated paper wax paper

g. Voternof cainats

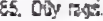

8. Asphat Ireases tirtent

Yy. Ar." cassiled above.

9. OTHES IYPE OF HATERLAL IENTED

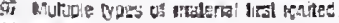

So. Na cizssilige atowa.

14.15 FOEU OF MATERLAL IGNTED

1. STUETLPAL COAPONERT, FIRISH

11. Exiefiar roal exvenna.s surtace. finsos

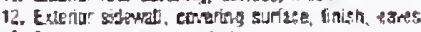

12. Doors, purches and plations.

Li, THe. Gerde, rug fooring and siars.

15. Intorion wall comring.

16. Cefinio comering surtace

17. Sinuctural menber. Iraming.

18. Thermal. acoustical insutation, willon wall or ceting.

19 riot classified above.

2. FUP.HTUAE

21. Uphoirted sola, croir wethirdo sents

27. Nonuthalstered chal, bench

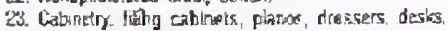
lutics and bookcases.

25. Vroning bourd.

25. Aszliance housing ar casing.

$20 \mathrm{Nor}$ cligsinited aborc

3. SCFT GOOOS. WEARIHG APPAPEL

31. Martes, tillow

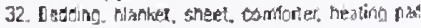

33. Lince tersists, tablectothe

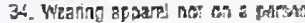

35. Weannz acparel on a femon.

35. Cin las l, yind dapery. bapesty

37. Fa:

3. Legage

as Hot ciassifed atcre 


\section{ABORNADN RECHEATLOHAL WATERTAL}

4. Christing tos

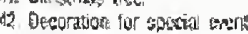

4. Burix,

44. Vagabise, nenepapez, witiag paper. 14us.

45. Toy, pame

afe tewring. canop

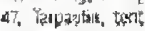

40 hial sasidied abom

5. SUPPUE, STOCK

51. Bax, camout, hist

5. Busbut carrel

53. Follet shid.

54. Fippe, cord, wine yom

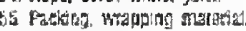

Sfi Bale ronge

5?. Heli stuphe.

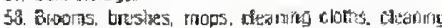
suphos

59. 20: classised athote

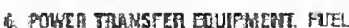

fin tiectrical whe, cable trisulation.

20. Trastiontar:

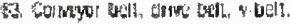

BA Tire.

05 fue

60 hal clastifex above

T. GEVERAL FUAM

7i. Articulabel prod wes.

72 torses pole

3 Fertälizer

7h. for crats. brush and ardse.

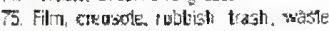

7. Covking materiak.

7 Sigh

8. SYEGIRL FER日

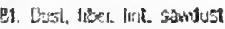

E?. Pyntechries oxplasives.

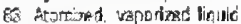

8c, Cong.

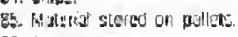

65. Pecsleratate

Q. Poiltés matena:

83 Ad'uesive

2. TTMEB FCHM OE MATELIAL

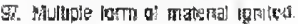

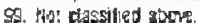

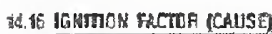

3. BHCESBIART

1.. Incemtary.

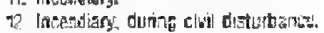

2. SUSPIODOW

2. S. STP

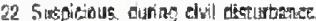

3. MISUSE OF HEAT DF IQNTIION

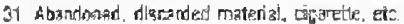

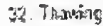

33. Folling aslow

3. Inted quate centel of spen hec.

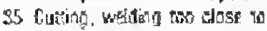

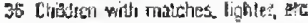

7. Unconscious, mertal, pliysical infatiment

39 Not claselfied abcre

4. MEUEE OF RIATEFIAL IRAITED

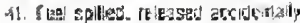

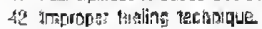

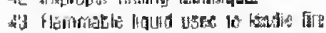

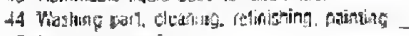

1.5 Innropes container

45. Combustibis zon rlose in trat.

17. Iriarager sluratue.

40 Not cilassilisa atron.
L MECHANICA MULBE, MLLURETION

51: Parl 1ailure, logk, Mreak.

52. Awtomatc comiol falure

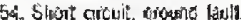

5 . Othe electrinal lealute.

56. Lact of maintenaxce. wom out laiture to whent.

57 exsifinis

56. Nat cossitiled ahm

6. DESIGK, CDNSTRUT:OR, HSTALIATOK BEFICIRNCY

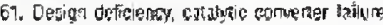

62. Coviryetha distitanty.

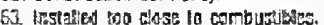

64. Other inssallation deticiansy.

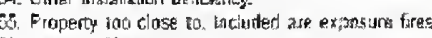

68. Nat dassitied arown.

7. DPERATHONA DEFCLRMCY

71. Collision, onstira, knockrdowa.

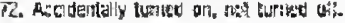

73. Lnattonded

7月 0ीvertand

75. Spontaneous haring

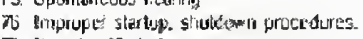

75. Nol classified atorte

3. Wat garal conotimon

EY. Misylu wark

E. Earinguke.

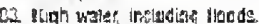

EA 1 inhtring

8. hol classhed abour

S. ITHER IGNITON FRTHPS

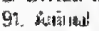

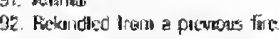

95. Nut classitied abure.

ae. No Fire.

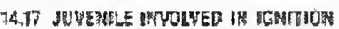

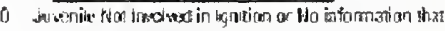

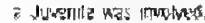

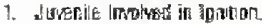

24 18 CONGERUKTION RI RSS

Cate Mo

C. No builderac imolied.

1. Parefing sirugare

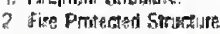

3. Nun Fixemout Strualure

4 Virod 5 rem: Struture

5. Angal Strunzure

6. Hezuy Timber Seructure

\subsection{ELASSIFICATION OF BULLANG BY USE}

COMACACHL

59 ? Dank

723 Bisurety

B95 frat Slotes

stit Dejoumme sion

61.5 Electrical Poner Plane.

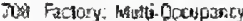

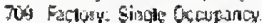

I31 Fusidy.

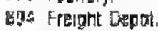

Bac Conge: Hea wibrage.

839 Gang: Stonse.

7 a Gas Whork Natisal Ger Piant

Q5i) Lumitac vara.

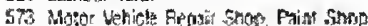

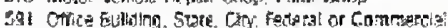

Sr1 ol soling sation.

AN1 Oil Storage Plant

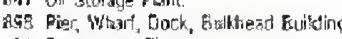

150 Rrgtautan: Dine

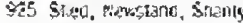

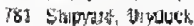

3is stable.

G14 Stam Gererating Plant
539 Starctuildisg. Txxpayer

29: Yiathouse siore

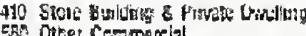

558 Otier Commantal

Pusua

171 Airbor Buturing

921 Erisate.

130 Churdi, Synzzopre.

I9 Oance Hal Dangur Hall

334 Displasany. Clinis

i7. Fary Yonmenal.

233 Hasplial. Intromant

315 Plursing Haiss.

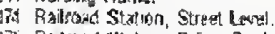

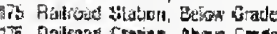

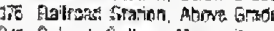

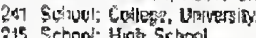

215 Sthon: Higat Srhool

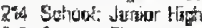

273 Sanow: Elemenay

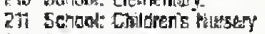

270 sechool: Othe

185 Television Stusio

IfT Theatre. Legitimatc

183 Theatre, Mation Pisure

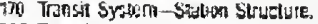

922 Tunnel.

Tis conter Pyotic

RESTDEATIAL.

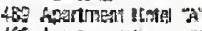

Thent tarece " $\mathrm{C}$ "

439 Jcarding House, Fooming Hinuse thi"

465 Comnit, Hectory, Blomastery, et

461 Darritary-semool, Coto, Irige

4 Al Pest ty:

430 Lodnimg Houe

40 Matel.

411 PPivis tomeling Ine Fansily

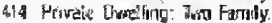

420 Tonement; Nex" Lat "

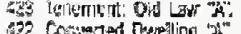

470 Uther Pesizential

SREGIAL PRTPEGTES

GR Airpon Eurhery

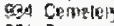

991 Constuction sils

S52 Derre. Landit.

931 Open Lat. Fialds

985 Pirkne pogs. Lot.

g53 Fipalian Prown lin fartin-ot Way

962 Preglic saper

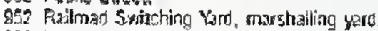

936 Verait Lots

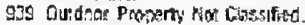

14.220 EUILDIHG STATUS COLE

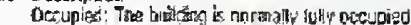
ur is intended to be tully occurpied A teav vacan ur is intanded to be tuly wocupied. A

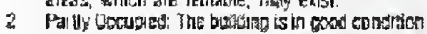
and more than ts parpert of the aress are vectant.

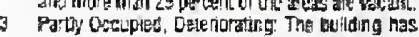

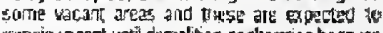

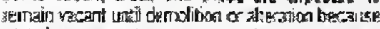
wi the' (a)

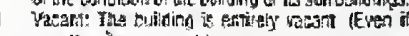

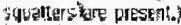

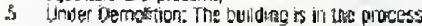
al being lam down

6 Under Constivelsos: Mle bualding is undu con. stuction and does not hawe any octupame

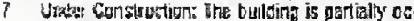

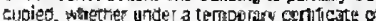
grcupanos of not.

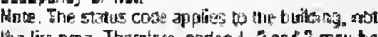

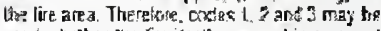

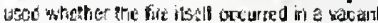
or cocursed area. snd coc 1 may arsly evan it

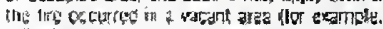
2 lire in a vach: apzoment terist repainted for

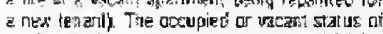
the fire aree is now recoltat on the 'Alse of Origi' Gode tree Pagnash 2.19.2 
4

พ.ะ ㅁตำ

11. PUSELC RECRIOADN COMPLEX

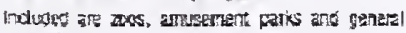
rezretion prites.

12. STRILAT EXHIITTON HALL COMPASX

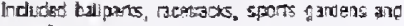
amonis

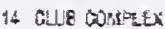

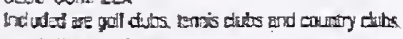

3) EDUCGTavin COMPLEX

Indidec are schools colloges and universitics

33 MEACAL CARE COMHLEX

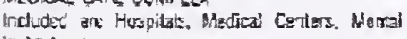
instristeris

34. PRSON Cosartex

4U YUSESSS WIT RESDEVTH COMPLD induted are apertrents uns stones

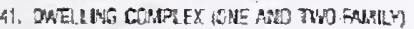

42 A

44. HUTL COMPLEX

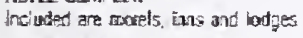

47. MOBLE HOMS: PLRK COHAPLEX

5e. SHOPPINE COMPISX

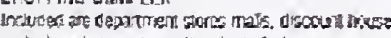

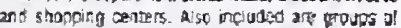

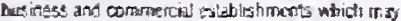

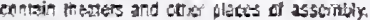

59. VEFICE COMPLER

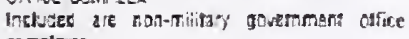
complaxes

61. PCHER PROUUTION LOMPLEX

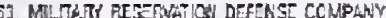

6.5. Than conserex

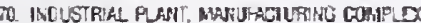

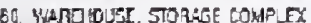

91. CONSTRUCTION COMFIEX

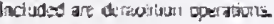

93 CAMPSIT COMPLEX

94 WHEGFFONI CUNALEX

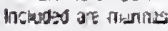

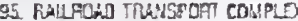

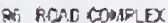

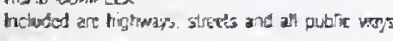

97. RIATORI CON'LE

38. HO CDMPLFX

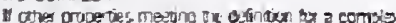

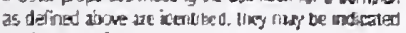
Dy Compita Cose 59 .

\section{FLOOR CODE $M$ O

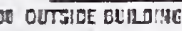 \\ 07 "st floor \\ to 10 \\ Sas $\sinh$ and Higher \\ S5 Arib \\ G5 $\sin$ \\ T) Bass ment \\ gT Basem \\ ori Cation}

AFE FIRE ORIGR

14.23 ARE FIFE QRIGITOCCUPARCT CLASSTERATIOR

no hor is EuHLING

COMMARACAL

Fastorr.

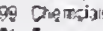

of Oresses

or Untergamera

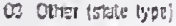

04 Ory Clazing Lavaeny

[e. ERecical Produets

of rood anr Mink Exodurs of Furntrua

od Fues, Fut Godat

of dens fits

70

71 lexhes lezine Epousts

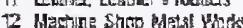

12 Paknis

14. Paper Products

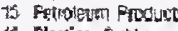

15. Flactios, Rubber

17 Priting and Alied industios

18 stoes

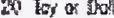

27 thex wy:k

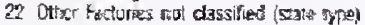
5 tore:

23) Aulo Accessuries

23 8akng

25 Eirotigr

26 Cands, Givar, Shatianary

27 Cimtaro

26 Dapartment, targe

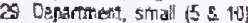

3C Dy Cearee \& Tolio?

3) D.

32 Eledical dogliresnce

33 Furs and vegetative

34 Furmatin

35 Frewers. Uairy. Debicatessan

36 Hzogrdastey

5) lacies hocessons

38 Laisty

39 Fins, Haxtrente

40 Pestavan: Lurcheoper

(1) 51:02

4 șinoc Fepis:

43 Supceratiknt

a: Tuper

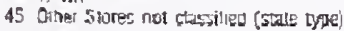
6rages:

40. Non starase

47. Sharage

45 gil soling Strian

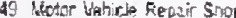

50 otice buising

Warrheuse:

51 Frr

52 Piper, Pags, Fiane

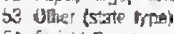

5. Fesigitin Deso:

35 Pier

Sof SMigeurs

5. Linter Yarg

5h Sined Hoxstand, sherti, atc

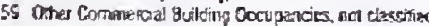
acroment Pesidential:

GG Apartivert. Ha:el, Bultiple Dugltung $x$

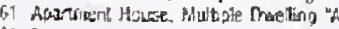

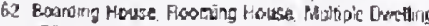
$T^{\prime \prime}$

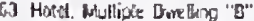

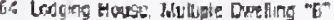

65 Private Dwelliris

6E Retersy. Canvent, Monastery. de

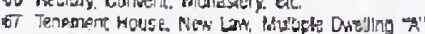

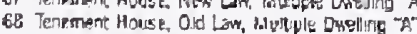

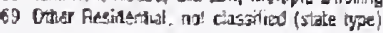

Pubre:

To hispert

71 Cabiret, bemcevel Hal

The Ehsirest

Ty nanse mall

74 klespirt

75 Metrot Pisture Treata:

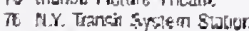

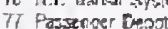

ir stical

64) IV Stuctio

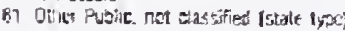

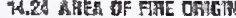

1. MENAS of ERPES

01. Hallwy, comorol male

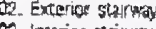

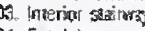

Q4. Escalator.

5. Lobty, endrense wrys

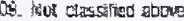

3. MSEEMGLY ARES

19. Fiund sects ciod ar mare perrons

22. Wund fixed seas d 100 or mane persons

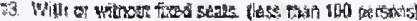

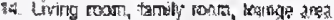

6. Seles, shrownomis arad

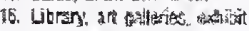

77 Swlaning

15. Nat chassified abowe

2. Futeriou atoss

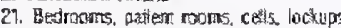

22. Wards, Gamitones, bancts

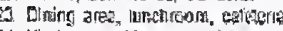

24. Kitchen, crohivg arce. diaknourn.

2. tatondry anee

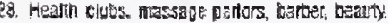

FUN AREAS (omationed)

\$1. Labaratery.

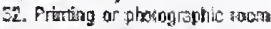

33. First ald, trezmens mom

32. Dperasin noum

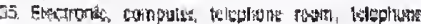
booth

36. Performante. Hege area, indoce spora

57. Projection Povat, statge Isht.

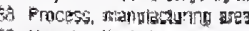

30 Not classitac abone.

a. FTIRARE ARE

a. Tank. bin, grosuct storaps room

42. Clases

43 Surdy raom,

46. Rexords sterage mosin. wall

45. Shipsing nocerving. Wadets nogit rown.

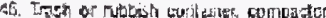

6. Garage carbot whtsin starage

39 Nat classilted' abore

2 SEFUICE FACIUTIES

2. Finator aut

5. Electriegl, alunatigg sentiation shate

59 Lipht shath.

54. Lundiy or mali chere

55 Duth.

56. Displisy wirden

57. Crimney five, stowepipe

52. Cornmor

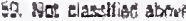

5. SETICE, EOUIRMEUT AAE

61. Wachinery mom

E2. Hiesting equipnent, wat Et heaper ares

E3 Sintitchoear arso, transforiner vatul

61. incinerator room wea.

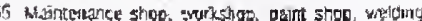
shop.

(a)

67. Engtwous with pressunzar at

68. Enciosure wint ennehed exyten anosprowe.

E9. Nor classified abrose.

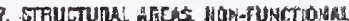

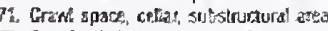

r. Exeriar balcomy, neren tootrit.

7. Fon and ceitug astembly concealed tooshating space

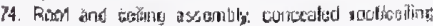
5 maces

35 Wall assumbly culleeafird wall space

76. Extgrour woll suriace

77. Exterwe west atriace

78. Approring, overthang.

7) Nel claserficé atmon 
6. TRANSPOAIRTOK, VEHICLE AREAS

81. Passenger area.

02. Inunk toad carnying arsa

Q3. Engine, ruming year wheel area.

84. Fuel lank, tael the

85. operating, control area, cab, cockgat

85. Exterior exposed surface.

89 Not dassified above

9. OTHER AREAS OF OALGN

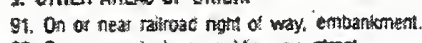

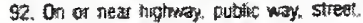

93. Terrate, patso, courtart

94 Lami, fietd open area. vacant lot.

g5. Wildiand area, woods.

97. Muthole losaton,

Se vacant room, apartment or are.

99. Not classified above

\subsection{MANWER OF EXTEHSTION}

\section{code the}

oo confined to area of orgia

07 cockdot.

O2 Doa a opening between rooms.

as Froot.

04 Hall. Stainary

as Pantican

06 Pipe Recess.

os Snat duntowaite:

os Shat Elevata?

of snatidi, Lethi, Chute Duct, etc.

10 Ceiling

10 Ceiling.

12 Othe? (5tate type)

14.26 MUTAER OF CCCUPANCES

\begin{tabular}{|c|c|}
\hline Codes & Descatiption \\
\hline or & occupancy \\
\hline 02 & 2 occupancies \\
\hline - & • \\
\hline . & * \\
\hline 99 & 99 or mare occuppencies \\
\hline
\end{tabular}

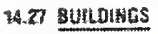

core: to 9

0.- did not soread terond buding of origin

1-1 structure or werucles

$\because$

$+$

9-9 or more butudings or vehicies

MUTE: Form Bf-24A must se submitted for eact buitding of venicie listat in thos coded space.

\subsection{WAMAOE COOES}

14.28.1 Percentage Cotes

0 Ho appreciable damage

1 From 1 through 159

2 From 16 through 494

3 30\% or creater

14.28.2 Extert of Damage Cades: To be used in whe Cumbe Category Baxes "Fiame Smoke and witer".

1. Cortined to objet of angin.

2. Confined io part of room or area of ongin.

3. Confinet to moon of orgin.

4. Corfined to fue rated compartment of onizin

5. Confined to ther of wign.

6. Coalned to stracture of oritar

7. Extended beyond the structure of orign.

9. No camage of the tyoc

14.29 SHDNE ANO HEAT DETECTOA CODES

14.29 .1 Present

1 Present
1) Not Preseff:
14.29.2 Fpe

$$
\text { 1. Srmake }
$$

2. Heat

14.293 Power Source

1. Battery

2. AC

14.29.4 Performance

1. In room of tre; operated

2. Not in room of fire; opartied

3 In room of fire; tid not opente

4. Not in mon of fire; did not operate

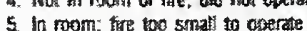

6. Oid not operate; powit source removed

9. Not ctassithed

14.3 POWEA FOR EOUPUEM

or 1-23 volts $A C$

Q2 24 wits $A C$

It $\$ 6$ vols $D C$

$12.7-12$ is $D$

15115 volts $A C$

200 wits $A C$.

30 220-230 vits $A C$

33231.330 wolts $A C$.

34331 of highter volis AC

$5025-50$ volts $A . C$

61 Butane

62 Coal, Coke charcoal, Peat

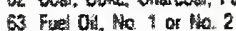

64 Fuel 0 , No 3 or Mc. 4

65 Fuel ox, No 5 a llo. 6

66 Gasoline

a Verosane

68 LN gas ystered as hquati

60 LP gas (groned to liquid)

70 matural of letumarang gas (as a gas)

7) Paper

7 Propane

99 othes

143I CODE FOR TYPE OF ACTOW TAKEH

1. Extinguishment

2. Rescue

3. Irvestination

4. Pemove hasard

5. Standoy

6. Savage

7. Firs Aad

g) Cance\#ted Enroute

432 CLASSIFICAIOH BY TYPE FRE OR EMERGENCY TRANSPOFIATION

Cose Ho

87 Ship Verset

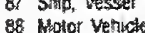

89 Other Transportation (state type)

\section{NOH-STRUCTLRL FIRES}

Cose Ha

84 Explosion, for attar fine

85 Outside Stalleak with Fite

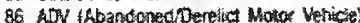

90 Bontire

91 Brush, Grass

92 Demoltion Woor, Bublding Site

93 Dutro Land Fit

84 Rubisth-Outside Building

95 uante

96 W.Y. Tansit System-Yard, Roadwry. Jies, etc

9 Radroad Yant. Roadway, Thes, eat.

98 tunnel. Bntoge

99 other Non-Strectural, mot classifited (state type)

\section{MMEAEENCY}

Couse He

G3 Eievator, Escalato

O4 Explosives Escont

of First Aid-Assist Personis:
06 First Aid-Resuscriation

77t Marine

06 Prberious Condition-Signts Trees, etc

og Subwar Railrood

10 Water Leak

11 tomb-unexploded. Scart

12 Colapse-Cave In

13 Collision-Veticutar hacident

14 Controlled fire Pernited

15 Flond Condition-Eraken Waler Mais

16 位cinerator

77 Leak-fuel Oil Gasoline etc

18. Leak-tituminating GaS. Fammable vapor

19 Liththing

20 oil Burner

21 Person Locked hn, Locked Out

22. Power Etectica

23 Pressure Rupture

24 fetrigerant loak

25 Sthoke Contition Gorn tounes

26 Sprinder - Leak, Water Discharne Damaged Head, et

26. Sprinder-Leak,

27. Steam Dectrarge

28 Defoctive Alam Devise (other then Sprinker)

29 Smode betector

30 Delective Alarm (Sprindder)-Surtye, Work on Systen, Other

1433 MOSILE PROPERTY TYPE CODES

it Auromobile

12 Bus

13 Hotarcycie, Snownobite

14 Motothome

15 Travel Frailer

17 Wobise Hom

20 Freugre, Road Transpont

30. Rail transpon

40 water Transpor?

50 Air Transpon

60 Heavy Eaupment

70 Special Vetictas, Containers

99 Onter Motile Property Type 


\section{Appendix D \\ SIGNIFICANT FIRES IN WTC 1}

Significant fire incidents occurring in WTC 1

\begin{tabular}{|c|c|c|c|c|c|c|}
\hline $\begin{array}{l}\text { Significant } \\
\text { Fire }\end{array}$ & $\begin{array}{l}\text { Incident } \\
\text { Date }\end{array}$ & Fire Location & $\begin{array}{l}\text { \# Sprinklers } \\
\text { Activated }\end{array}$ & $\begin{array}{l}\text { \# Standpipes } \\
\text { Activated }\end{array}$ & Cause of Fire & $\begin{array}{l}\text { Material } \\
\text { Ignited }\end{array}$ \\
\hline 1 & 9/9/77 & $\begin{array}{l}\text { B-6 level storage } \\
\text { room }\end{array}$ & 2 & & None listed & Not listed \\
\hline 2 & $9 / 23 / 77$ & $\begin{array}{l}\text { Dumpster on B-4 } \\
\text { level }\end{array}$ & 2 & & $\begin{array}{l}\text { Not } \\
\text { classified }\end{array}$ & Trash/waste \\
\hline 3 & $10 / 16 / 81$ & Floor 19 office area & - & 2 & $\begin{array}{l}\text { Discarded } \\
\text { material }\end{array}$ & Furniture \\
\hline 4 & $12 / 23 / 83$ & $\begin{array}{l}2 \text { dumpsters on } B-4 \\
\text { level }\end{array}$ & 2 & 1 & Suspicious & Trash/waste \\
\hline 5 & $1 / 27 / 85$ & $\begin{array}{l}\text { Office space on } \\
\text { mezzanine level } \\
\text { (floor 2) }\end{array}$ & 2 & & Incendiary & Trash/waste \\
\hline 6 & $9 / 10 / 85$ & $\begin{array}{l}\text { Garbage dumpster in } \\
\text { service elevator } \\
\text { lobby on floor } 43\end{array}$ & 2 & 1 & Suspicious & Trash/waste \\
\hline 7 & $11 / 1 / 85$ & $\begin{array}{l}\text { Storage closet on B-4 } \\
\text { level }\end{array}$ & 3 & 1 & Suspicious & Supplies/stock \\
\hline 8 & $6 / 7 / 86$ & $\begin{array}{l}\text { Dumpster fire on } \\
\text { floor } 106 \text {, compactor } \\
\text { room on floor } 107\end{array}$ & 2 & 1 & None listed & Trash/waste \\
\hline 9 & $9 / 30 / 91$ & Office on B-4 level & $\geq 1$ & 2 & $\begin{array}{l}\text { Discarded } \\
\text { material }\end{array}$ & Trash/waste \\
\hline 10 & $11 / 19 / 91$ & $\begin{array}{l}\text { Electrical closet on } \\
\text { floor } 93\end{array}$ & 0 & 2 & Short circuit & $\begin{array}{l}\text { Electrical wire } \\
\text { or cable } \\
\text { insulation }\end{array}$ \\
\hline 11 & $7 / 23 / 92$ & $\begin{array}{l}\text { Level B-5 at the } \\
\text { power distribution } \\
\text { panel }\end{array}$ & 0 & 2 & $\begin{array}{l}\text { Electrical } \\
\text { failure }\end{array}$ & $\begin{array}{l}\text { Electrical wire } \\
\text { or cable } \\
\text { insulation }\end{array}$ \\
\hline 12 & $11 / 10 / 99$ & $\begin{array}{l}\text { Computer room on } \\
\text { floor } 104\end{array}$ & 3 & $\geq 1$ & None listed & $\begin{array}{l}\text { Plastics, } \\
\text { electronic } \\
\text { equipment }\end{array}$ \\
\hline
\end{tabular}


KETUKI- IKULIUKAL FIRE
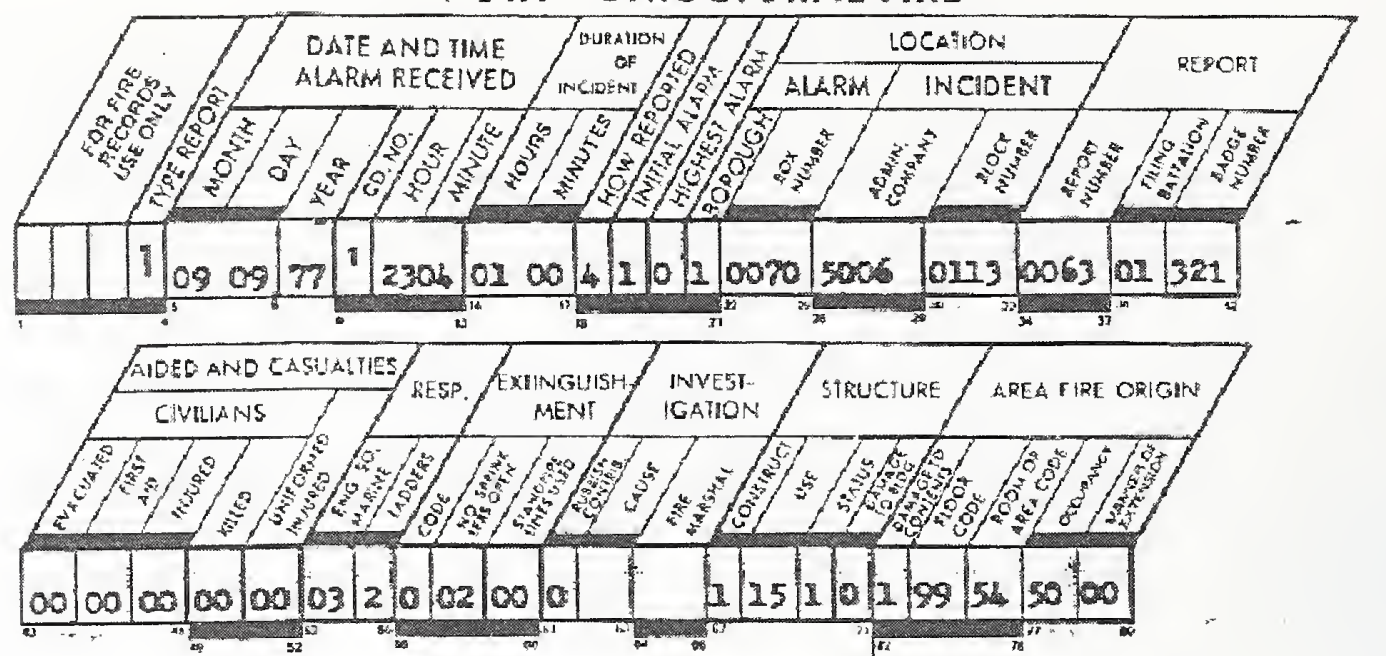

\begin{tabular}{|c|c|c|c|c|}
\hline & 1 & ward & Preda Center & Kang \\
\hline & NUABEER & & S7aEtT & BOROUGH \\
\hline & $2:$ & 10 & $200-200$ & \\
\hline
\end{tabular}

NAME OF OCCUPAND TODOATAPT ND

Srt. Dasal P. Pollo IEFIN CMAREE

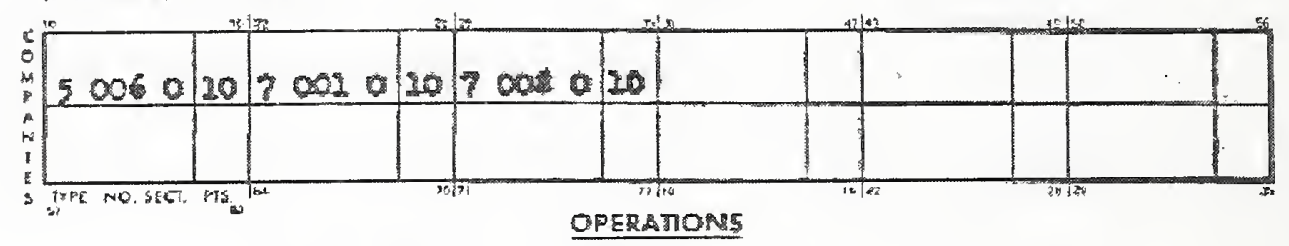

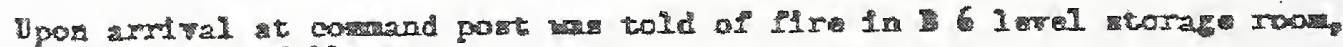
operations as 20110w.

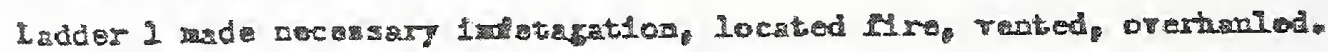

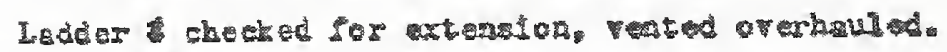

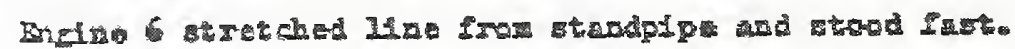

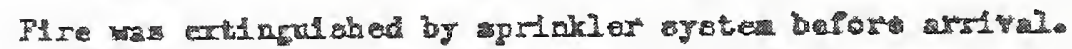

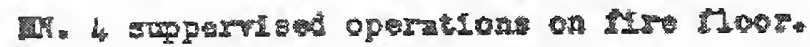

Fire patrole 2 on the acese.

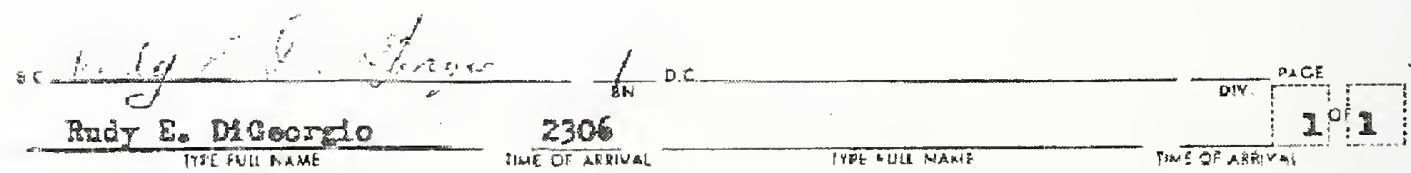




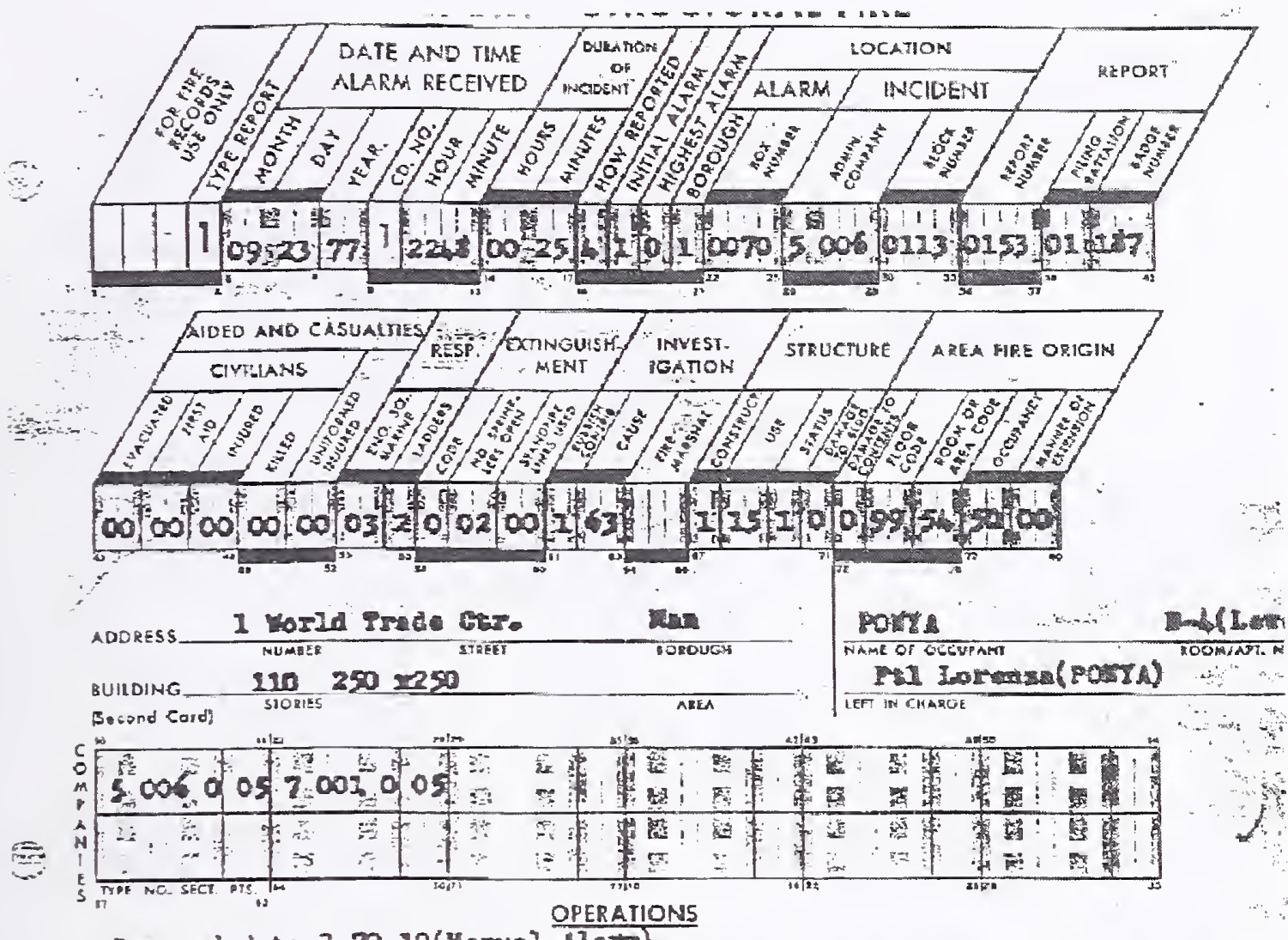

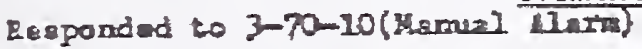

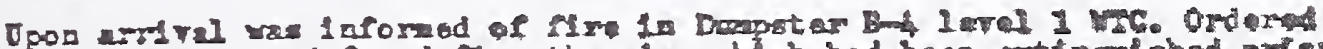

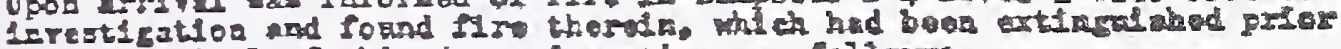
to the arritral of this dept. Operations an follores

Be 6 Rollod up lanctis staod last.

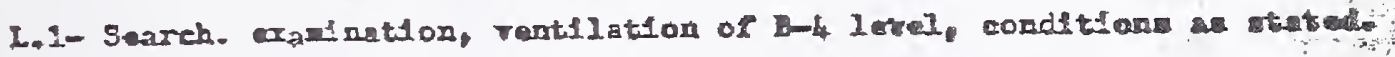

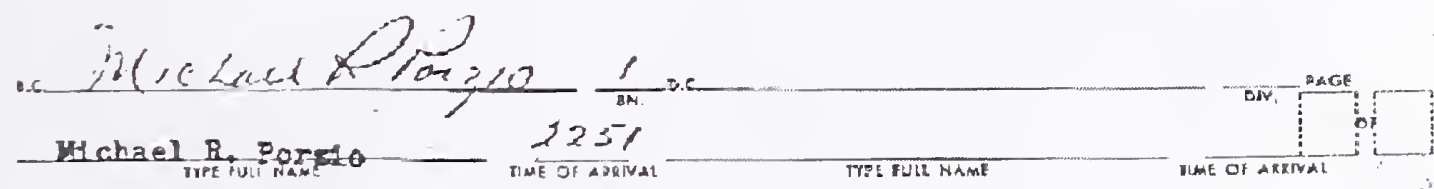

AOMINISTRATIYE COMPANY 


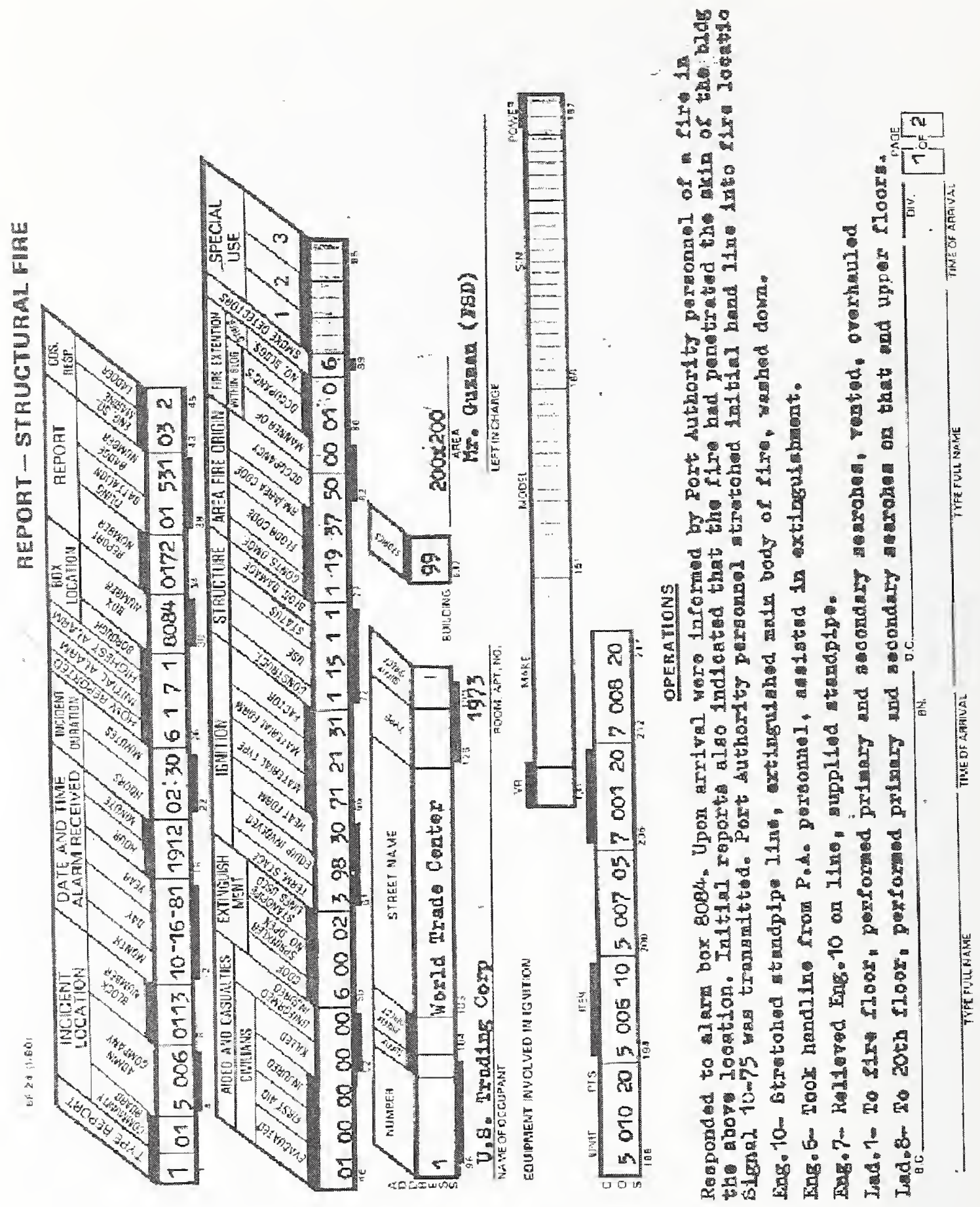

ARMUYSTRATIVE COMPANY 


\section{- STRUCTURAL FIRE, TRANSPORTATION FIRE, NON.STRUCTURAL FIRE OR EMERGENCY}
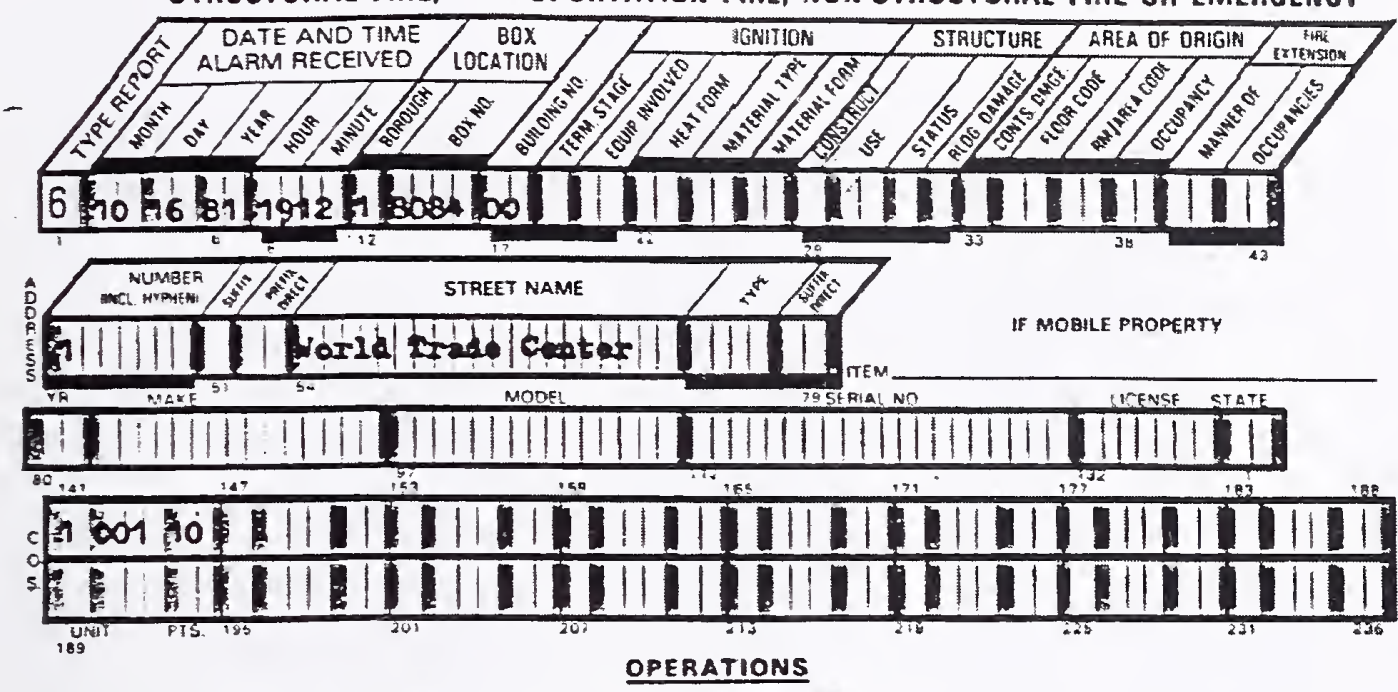

Lad.8 (cont.) - Poreed door to Orfice rookt2073

Bos.1- Checked rest ducts and stelwags on and above fire Noar, ascurnd pessenger elerators sarrias IIr Hoor.

Patrolt1- On scene, sulvage vort on 16tb and 17th floors.

Patrolt2- On scene, selvage vort on 18th floor.

D2T.1- D.C. Ross1 an sceno, in charge of Department operations upon arrival.

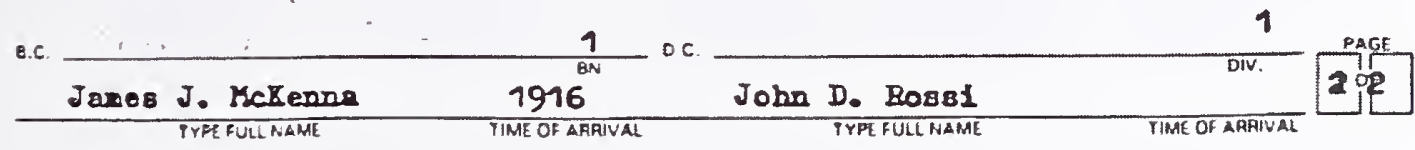




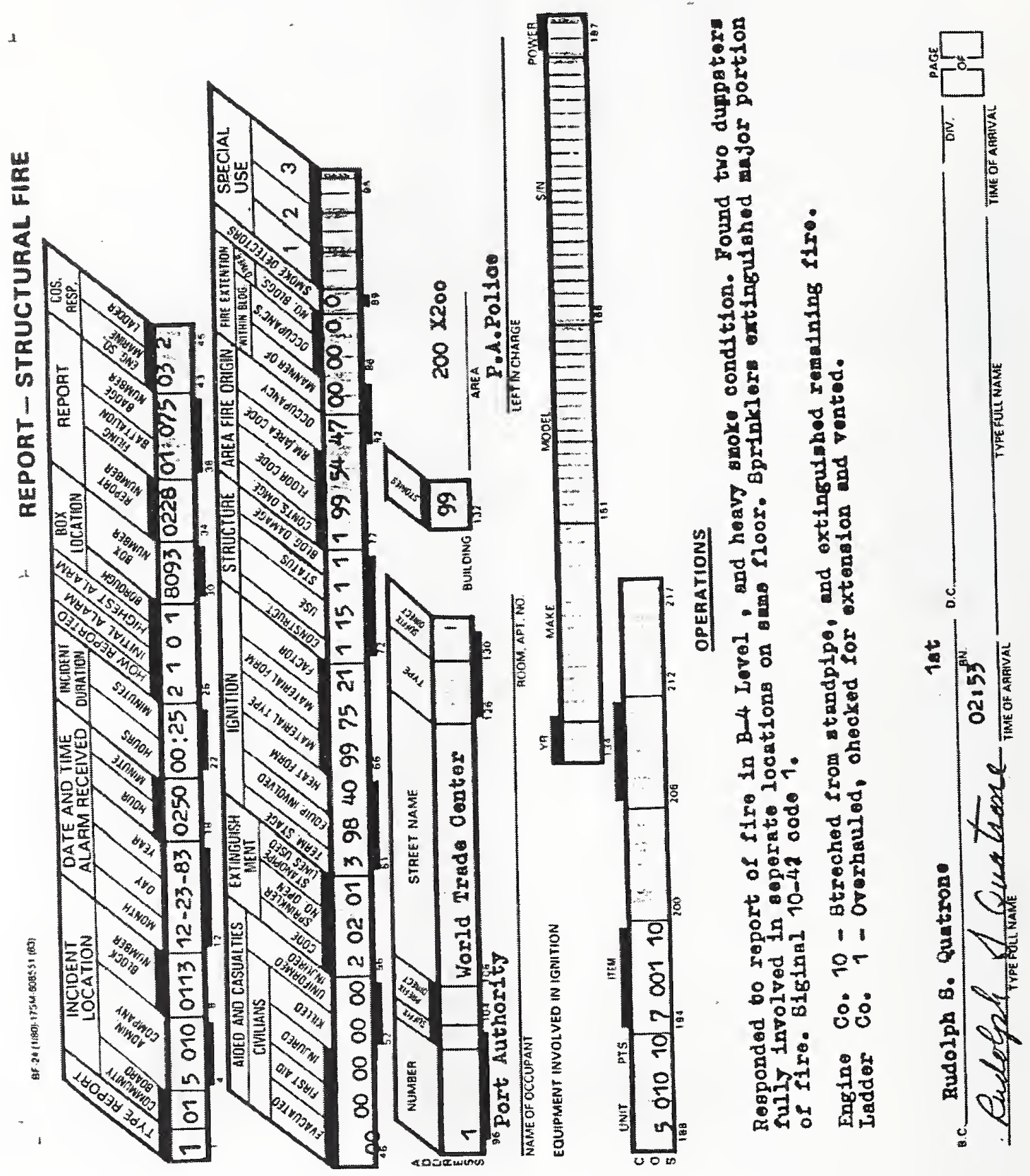




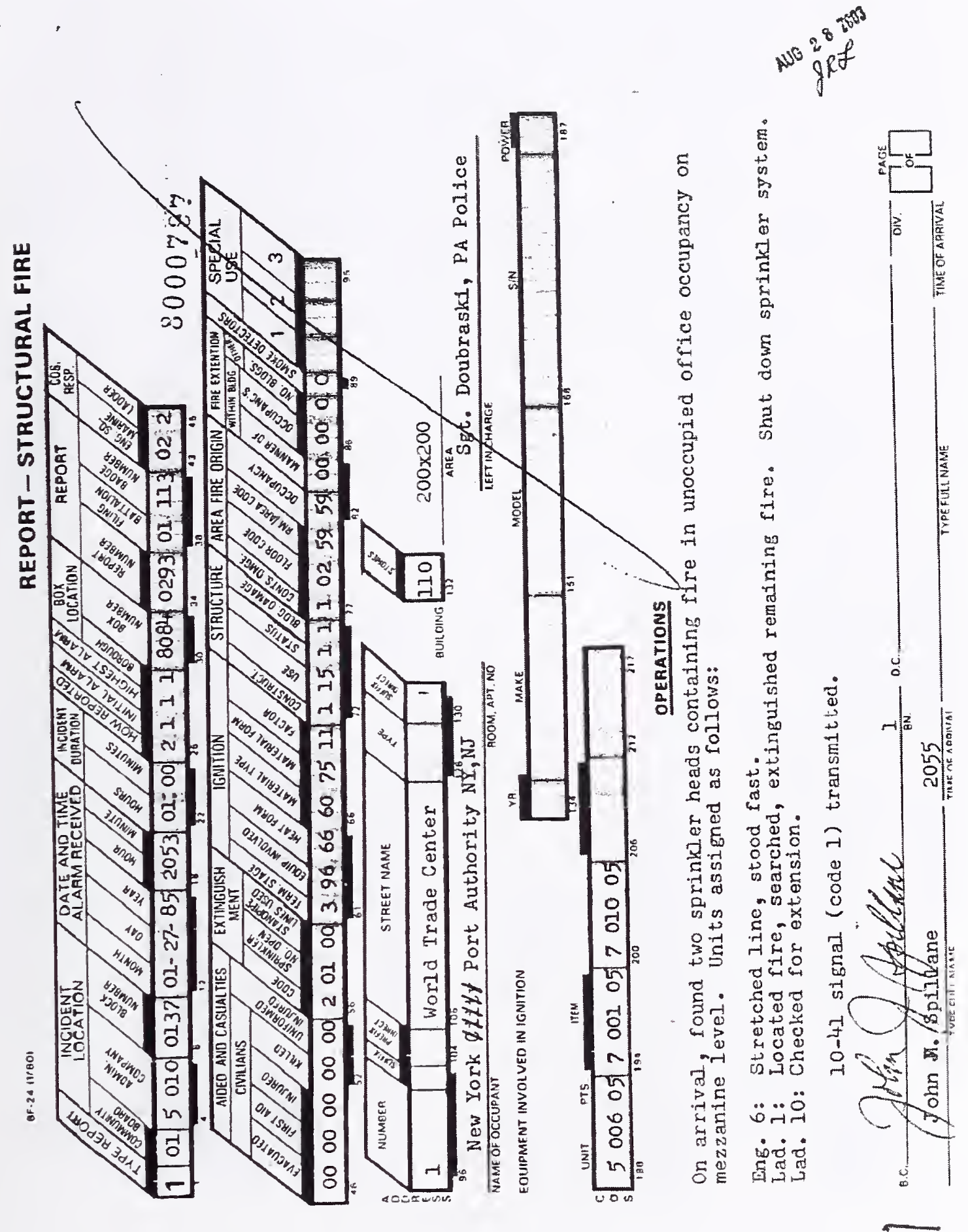




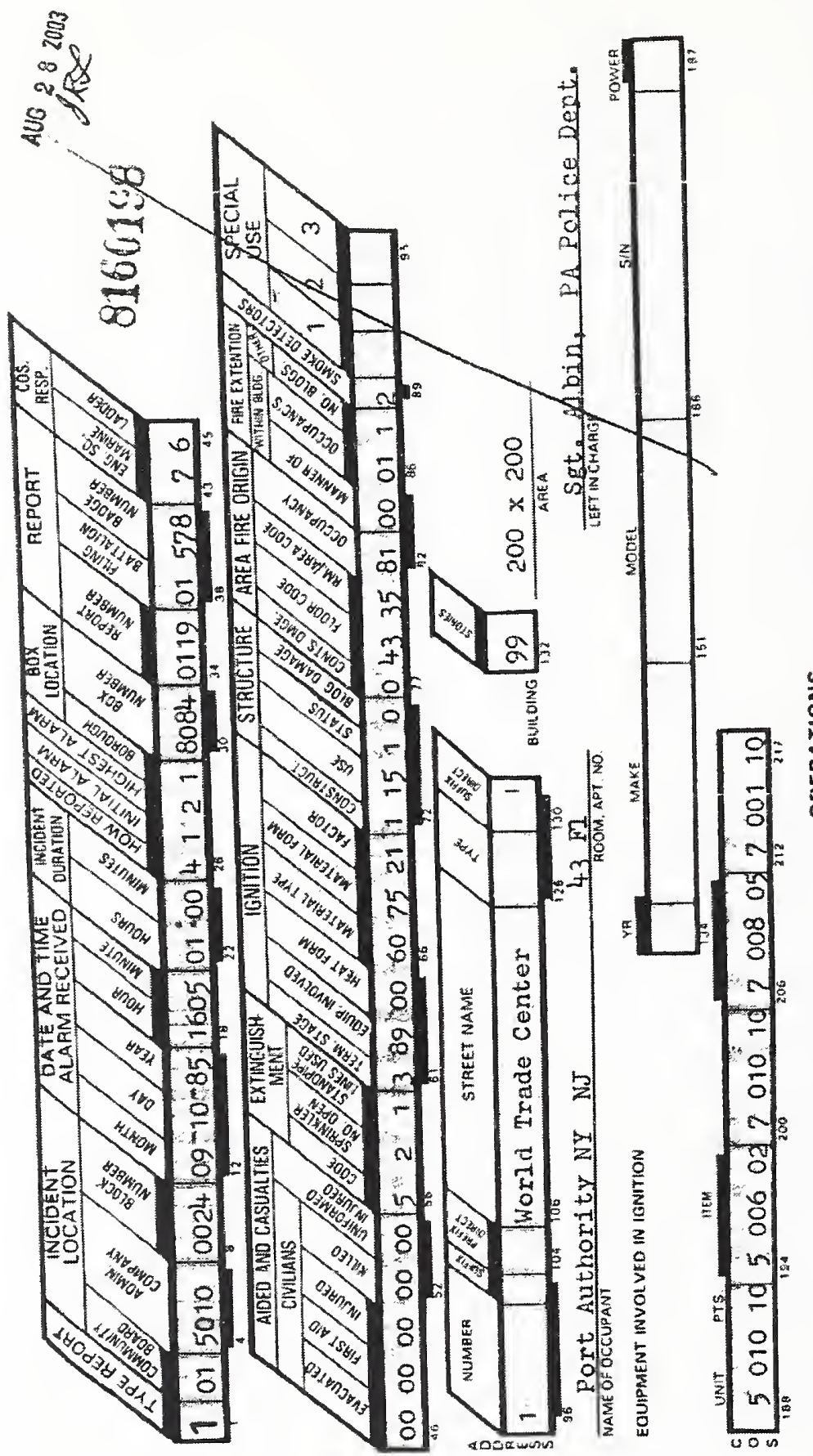

영ำ

न

政

s.7.

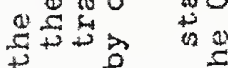

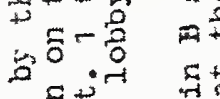

명

Q

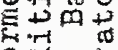

얼

$30 \%$

च 4

3 m.

- in

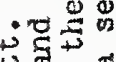

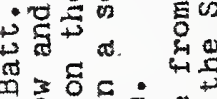

年

स्त 5 웜

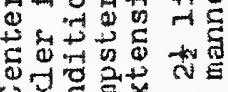

ต ब.

?

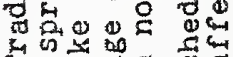

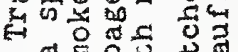

का क⿺⿻一𠃋十

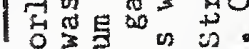

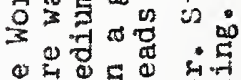

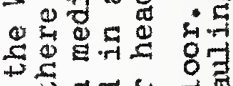

出

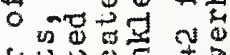

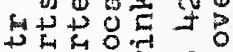

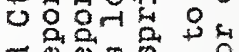

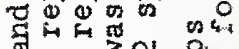

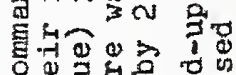

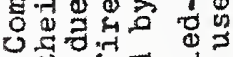

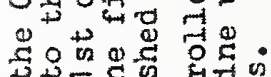

स

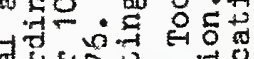

तुद्युज तु

$>001 \%$ ó

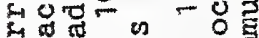

वैल क्षै

도영

过嵒

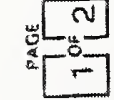

$\checkmark \mid \frac{2}{0}$

$\mid$ 粱

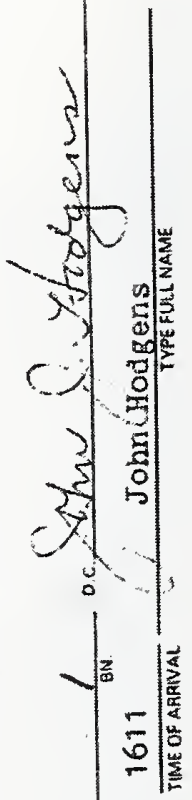




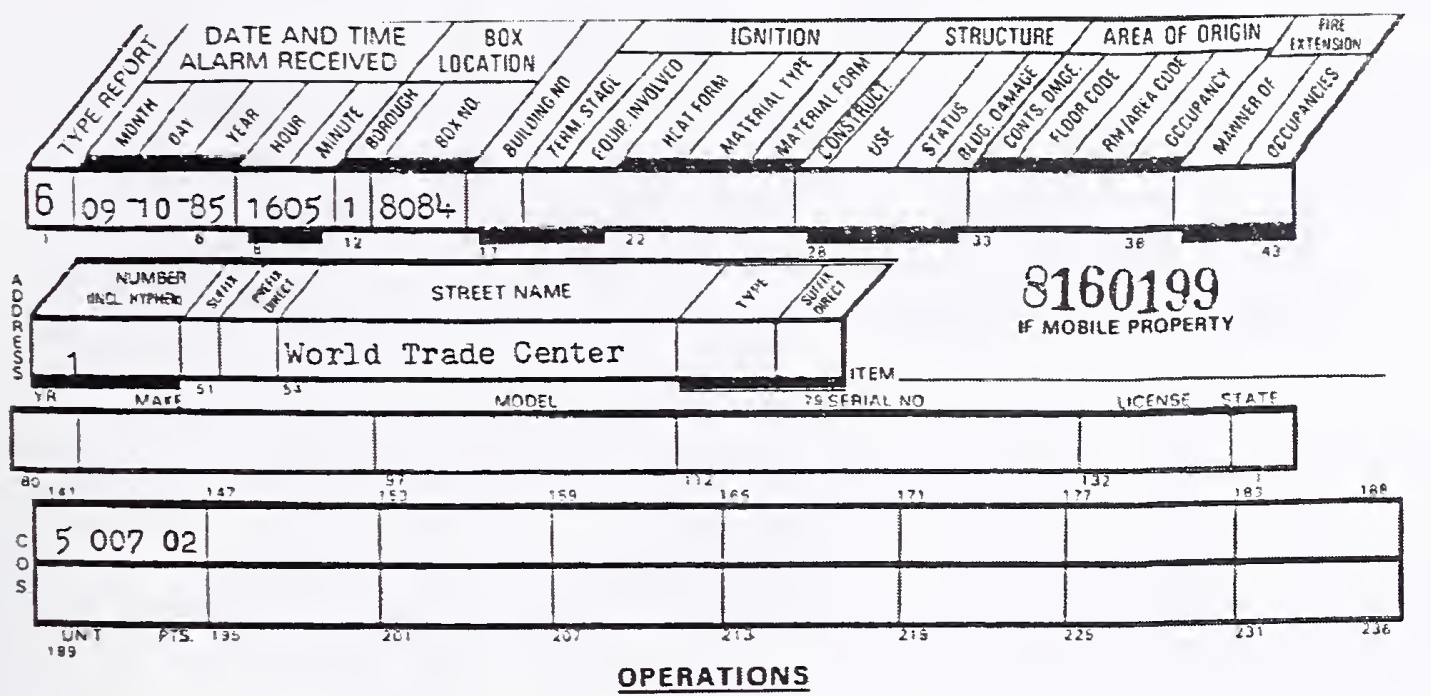

Engine 6- (teamed with E.10) Assisted E. 10 in Standpipe stretch.

Engir.e ?- Manned the Standpipe phone in Stairway B on 1 st Fl., relayed messages to and from Cormand Center

Lader $10-$ To $43 \mathrm{II}$, stairway $\mathrm{B}$, found fire, searched $43 \mathrm{fl}$ (negative) made neessary examination and overhaul of fire area.

Ladder 1-to $4 \mathrm{n}$, searched for fire, smoke extension, searched for troubled occupants (negative) Checked top ten fis (101-110) for smoke (Neg.)

Ladder 8- Made secondary search of 43 fl (negative). Searched fis 44 to 49. Searched affected service el evator cars \#17 \& 29.

Batt. 4 - Set up operations post on $43 \mathrm{Fl}$. Communicated with Comand Center, supervised operations of units on 43 and $44 \mathrm{fls}$

E.4, E.3 (HiRise Unit), I.15 \& Res. Co 1 stood fast, took up.

Brooky cos responding on supplemental Boxes 9031 \& 9032 at 1617 hrs Batt 31,

Batt 32, 2. 205, E. Xod 279, L.101, L.110 stood fast, took up.

F.C.U. - Established Field Hitrs, Monitored Communications.

Sprinkler heads (2) replaced by P.A. personnel.

Signal 10-41, Code 1 transmitted, Evidence of separate, previous fire reported to FM McCaffrey.

Responded to Scene: AC Matthew Farrell, Manhattan Borough Commander

$\begin{array}{lll}\frac{\text { Signa? }}{10-84} & \frac{\text { Time }}{1609} & \text { By } \\ 10-76 & 1615 & \text { Batt } 1 \\ \text { P.W.H. } & 1627 & \text { Div 1 } \\ \text { U.C. } & 1640 & \text { Div 1 }\end{array}$

t

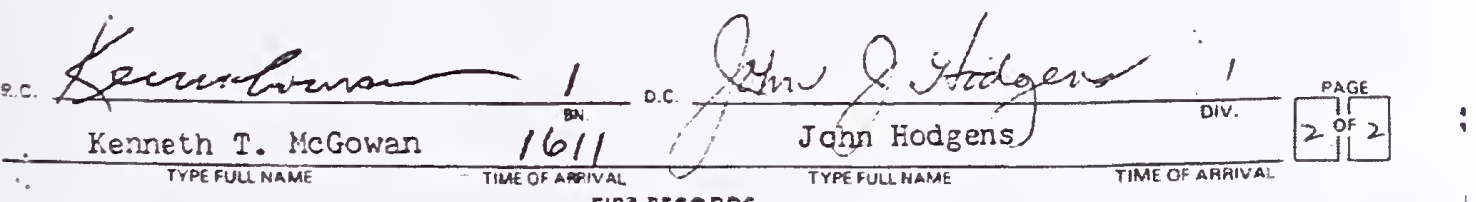
FIR? RECORDS 


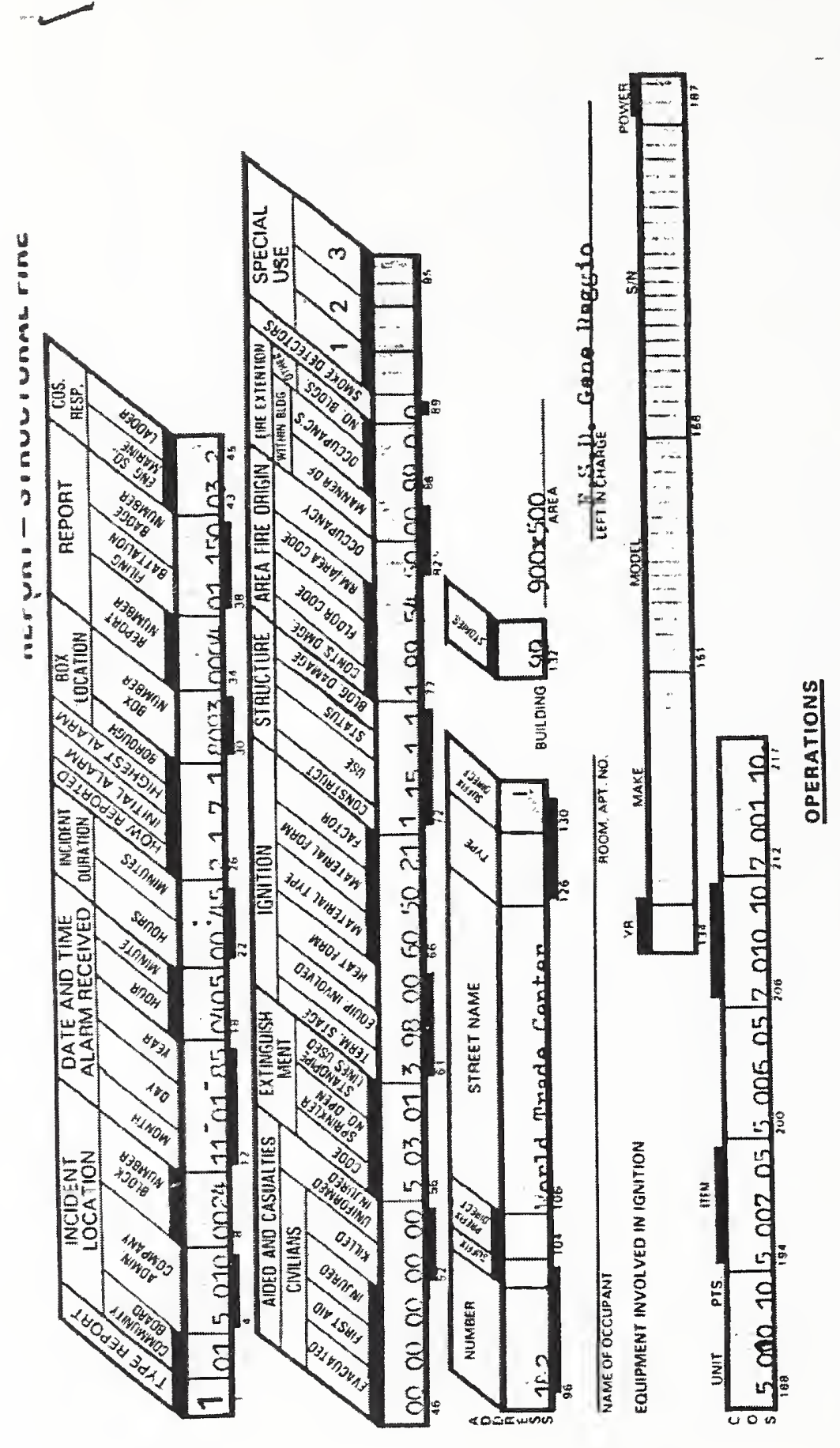

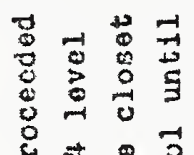

落

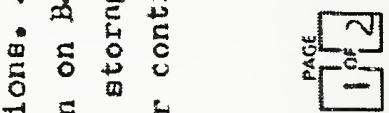

式起

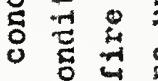

월 4

范造造

> $3:$

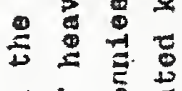

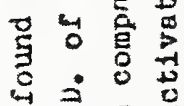

충 웅

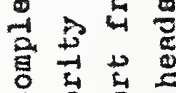

예월

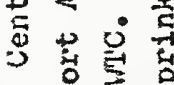

언

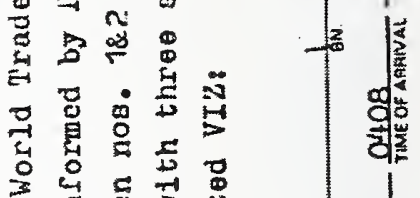

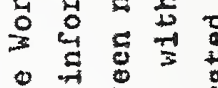

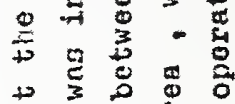

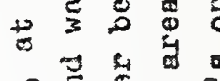

ल है है

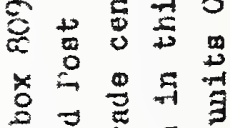

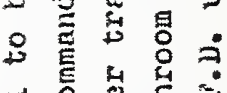

ङ.

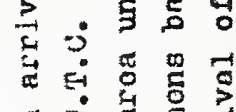

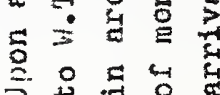

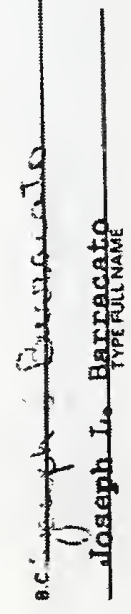


RETUN: - MUUIIUTAL UAIA

STRUCTURAL FIRE, TRANSPORTATION FIRE, NON-STRUCTURAL FIRE OR EMERGENCY

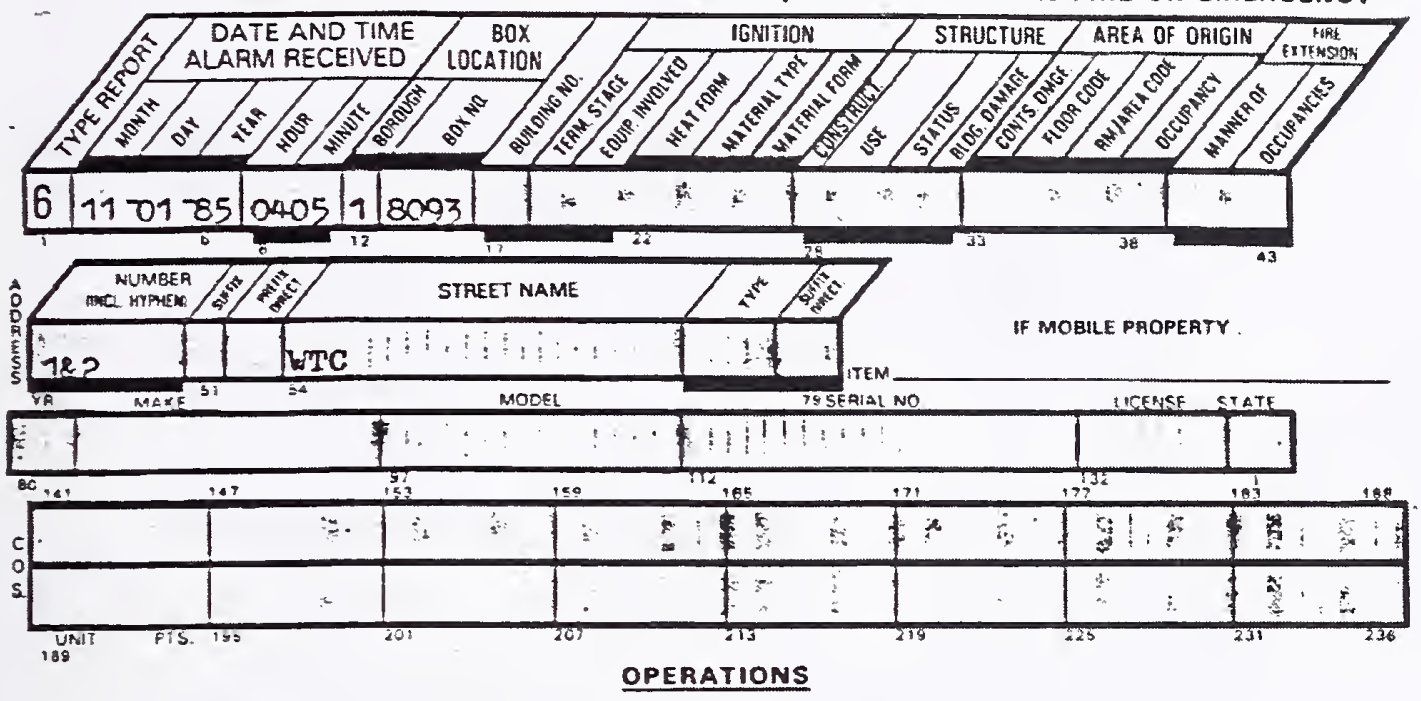

E-10 stretched line into fire area from standpipe and extinquished all rewaining in closet area

in? Etretched line from opposite side of fire and stood fast

-.E assisted 210 in stretching line and relieved on line and then washdown

I-1C found fire and perforsed necessary vis and overhaul in area. mace frimary and secondary search, then up

In perforbed necessary Vis and checked for possible extension in surrounding areas

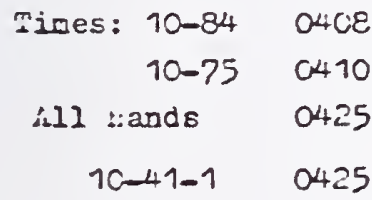

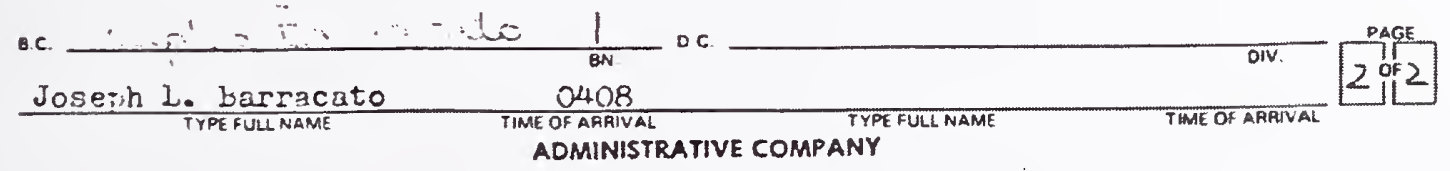




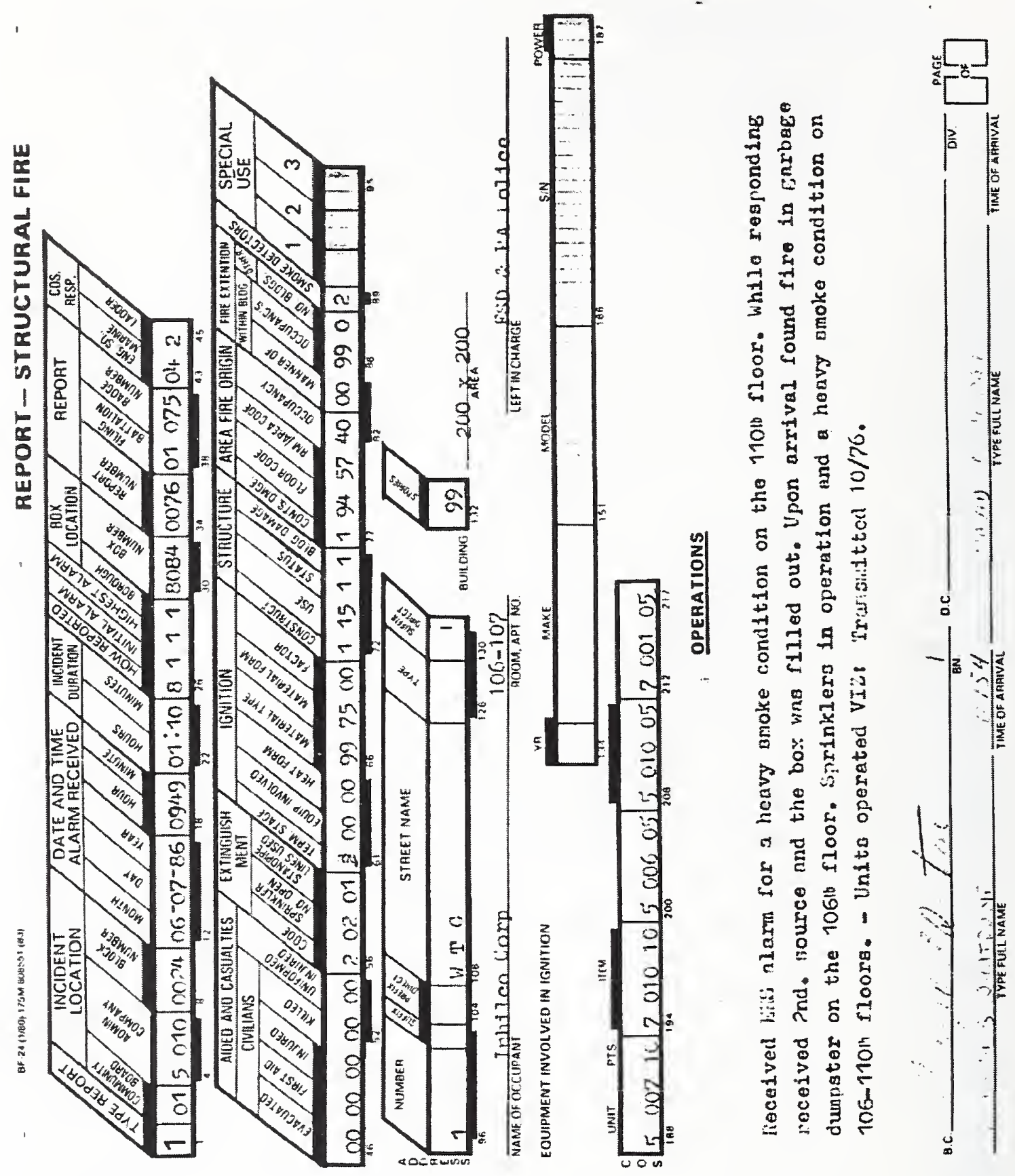


HEPORT - ADDITIONAL DATA

STRUCTURAL FIRE, TRANSPORTATION FIRE, NON.STRUCTURAL FIRE OR EMERGENCY
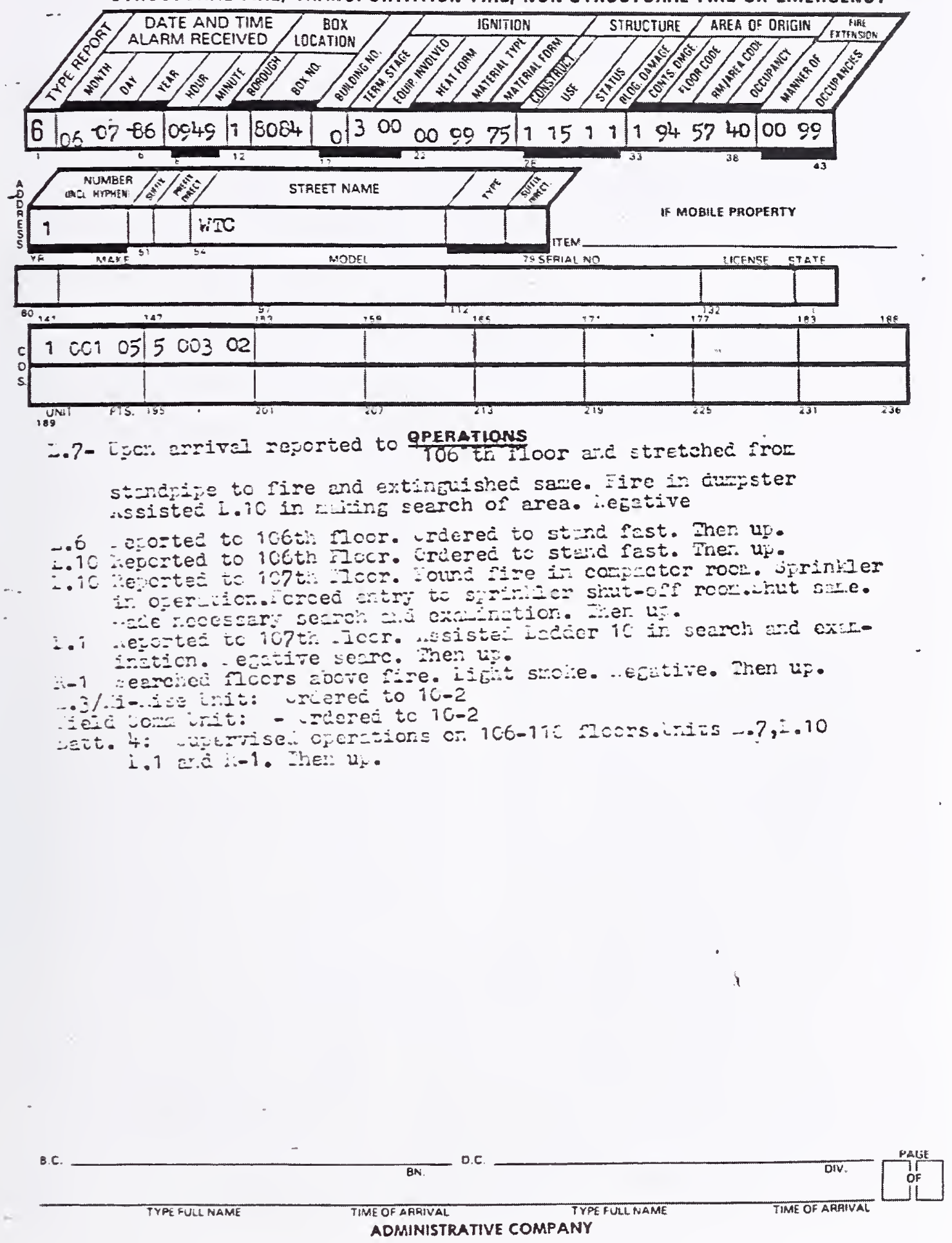


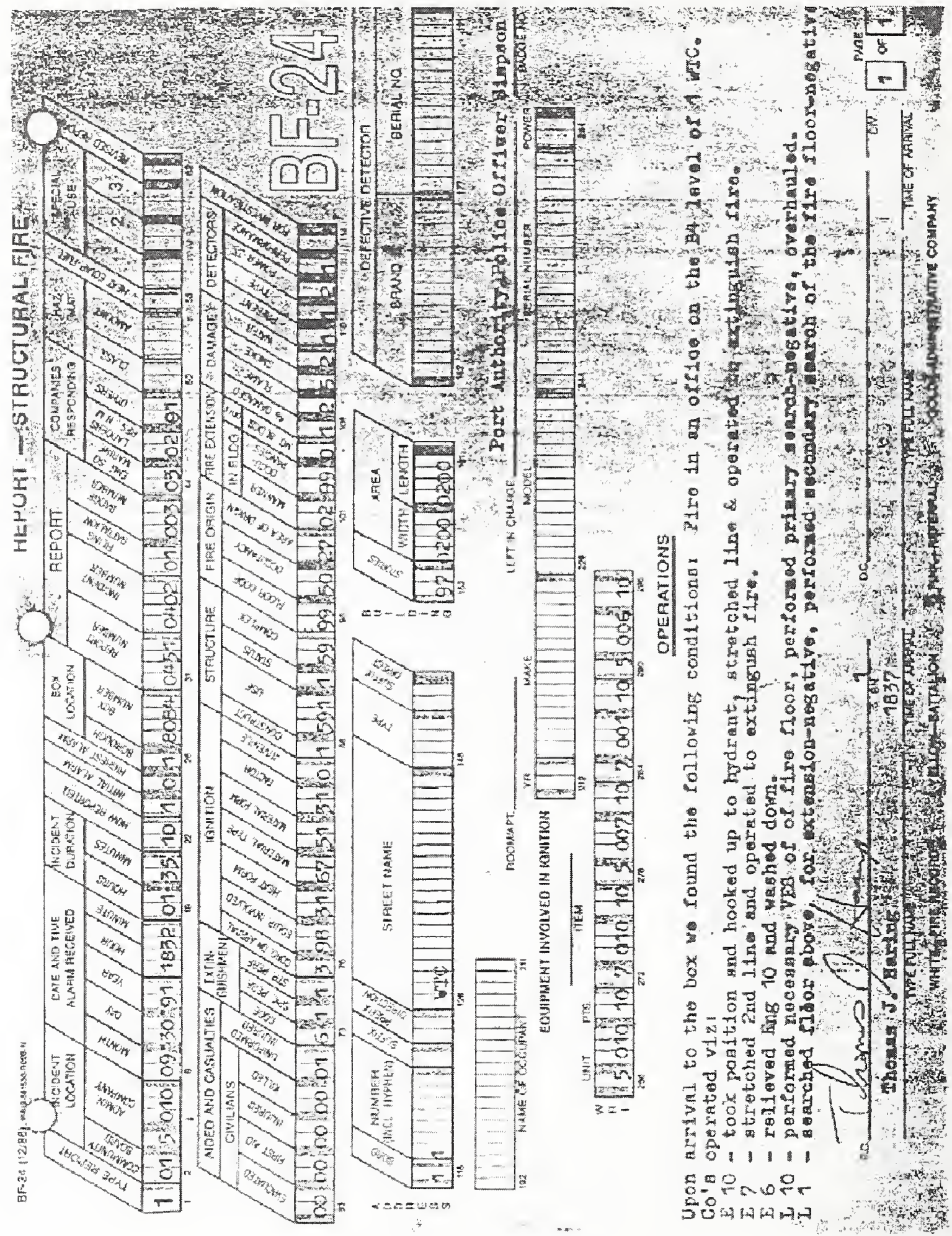




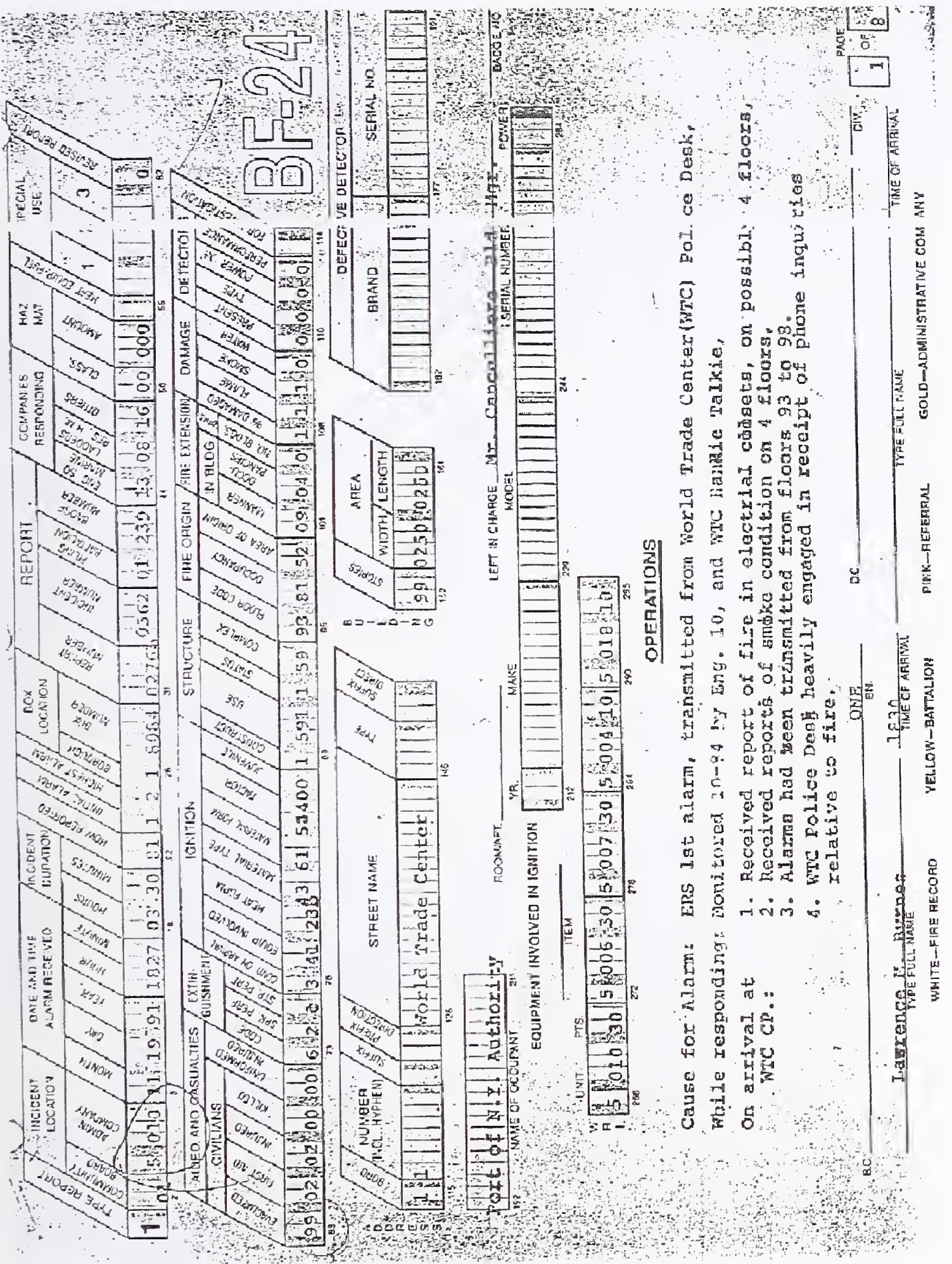




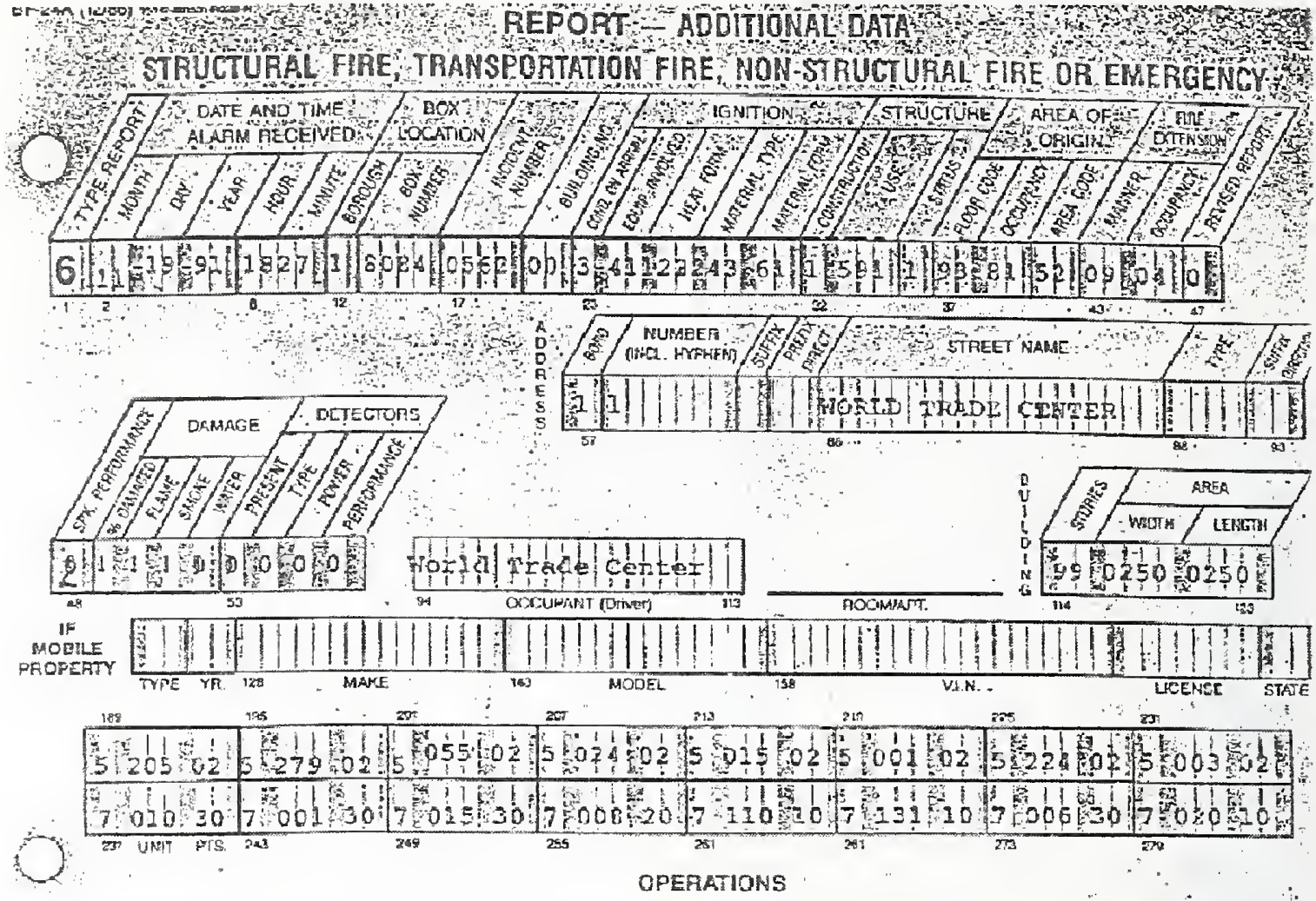

SThinEGY: To ettempt to contain the fire dituetion to electrical elorets, ane to prevent pante anc hysterle in accupenaies with large ojuilian populations, and establish parameters of fire and smoke condition ae quálokly g possible.

Action talien:

1. On aIxival at WTC cominat post, asauned conmand of Fire civuation,

2. Eornituidated with wadar co. 10 mho conelmed fire situationon Iloors $93,94,95$ and posslbly on 96,

3. Orkexed the transtission ot eignal 10-76 jased on: potestial for large volume of Exre, height to fixe axea, time invoived in units response, dificulty in cffecting good voice comunteatloss in building,

4. Orjered Huntatea Dispatchex to iext 10-76 arxivirig units to assemble in lokby stiging. axea, pending unit assigrineste

5. ordered firgt alan units to conouct ajijgent search.

5. Ordered Ist alazm Englne compenies to stretch from the "A" stairwell, but not to use-water until" confrimation

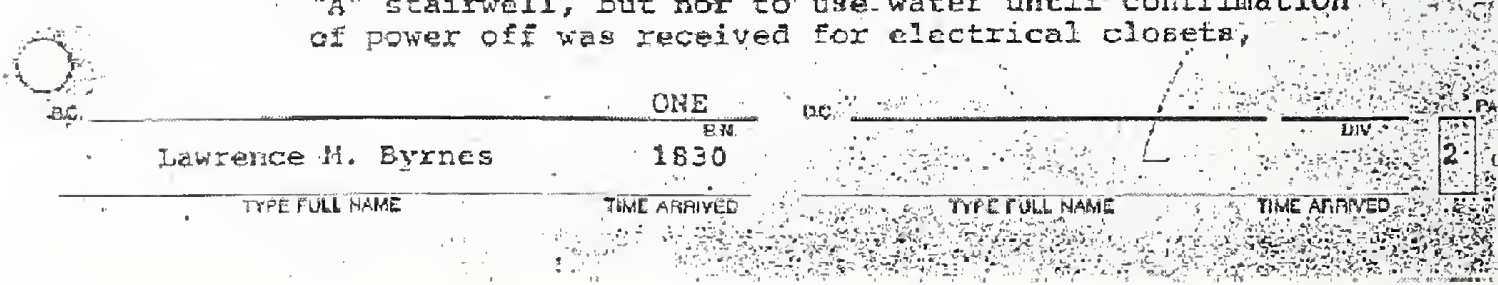




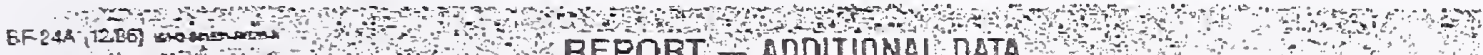
REPQRT ADDITIONAL DATA

STRUCTURAL FIRE TRANSPORTATION FIRE, NOH-STRUCTURAL FIRE OR EMERGENCY

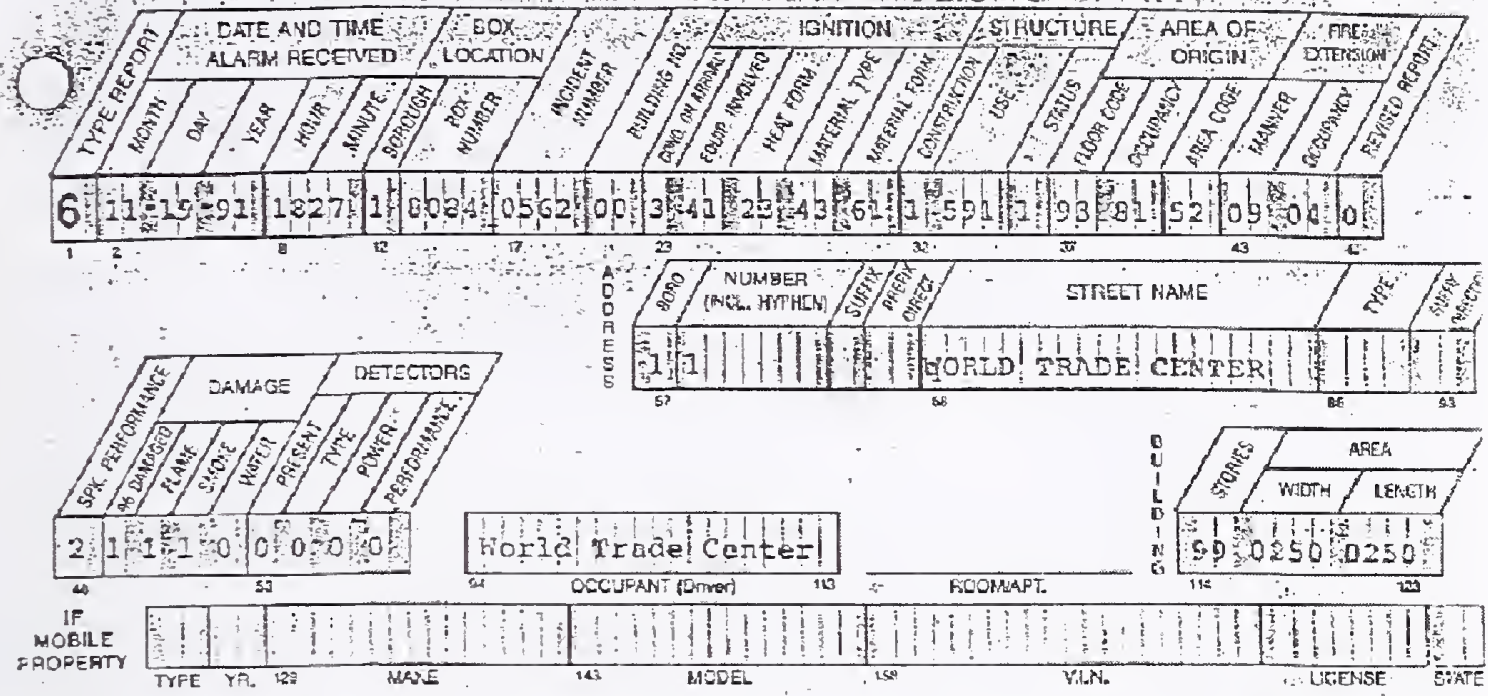

(3)

OPERATIONS

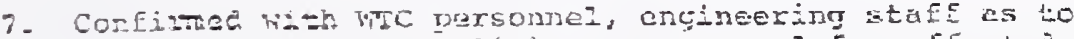

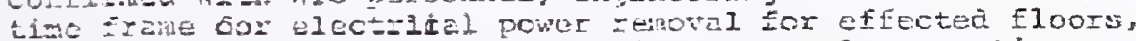

e. Denied WrC juices request to initate general avacuation

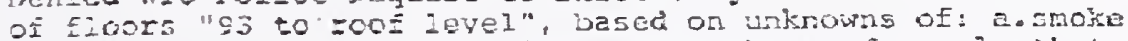

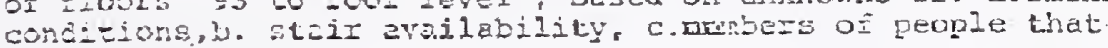
ney le is.vives.

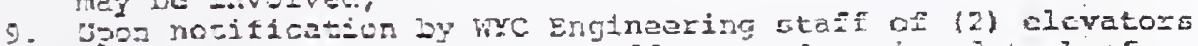
síuck at écos EE ETC 101, Jadder Co. G eseignaù task of victte zeroval, as accompanied by hic slevator recherde,

10. is lhief cfficcre extivec at commanc post they were issued INTC Handie ialkiss ane thely fsgigmentos

11. Called for Ejis zosponse for reported burn victim incchanic with fariel buras and for pessible neGds,

12. Irimeti search of upper flovrs praved to be time consuming due to large floom aree and initer tandie galkie capubalitias, consucted by 13t alerr truclis.

13. Foscuo Compary i assicned to follow wp eccomary search of

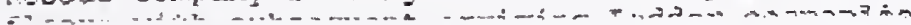

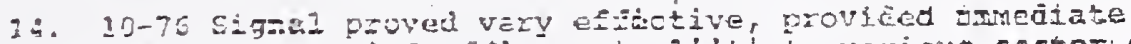
rcsonse o: chief officers to ititiate vazious scetor cortrols.

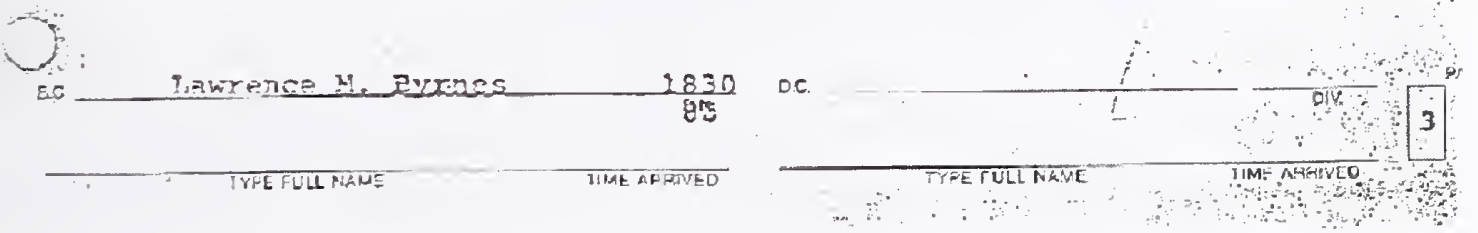




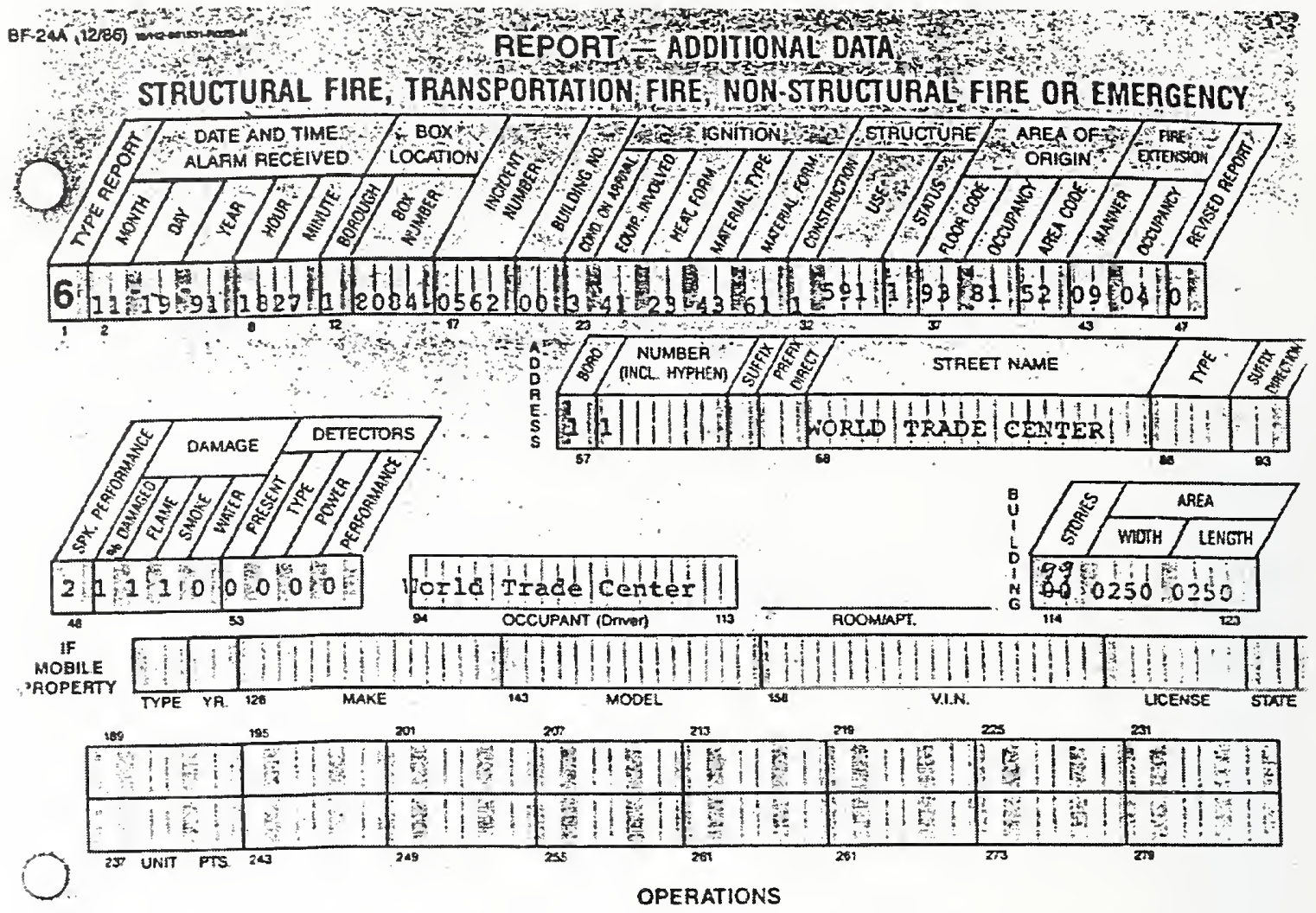

Chief ofticeis at scene:

DAC. E. Eutler,..
DC. H. Meyers
DC. V. Dunn
E.C. I. Eyrnes
B.C. R. Arclsson
B.C. Davison
I.C. Roche
B.C. Shelley
B.C. Hagner
B.C. Dawe
B.C. Cesark

CiA Duty,

Div. 1,

Div. 3 ,

bett. ONE,

Batt. 2

Batt. 4,

Batt. 6,

Batt. 31 ,

batt. 32,

s.c. Cesark

Batt. 7,

Safety Battalion.

Personnel present at Scene:

P. Sellbizer Fire Coma. Laison,

D.C. Basile Eits Supervisor.

n $n$. Tim यक्याn

Lt. Valle HYPD PCt. 1 supervisor,

Mr. T. Canceliiere WTC Blag Mgr.,

(2) Representatirue of salvation Andy.
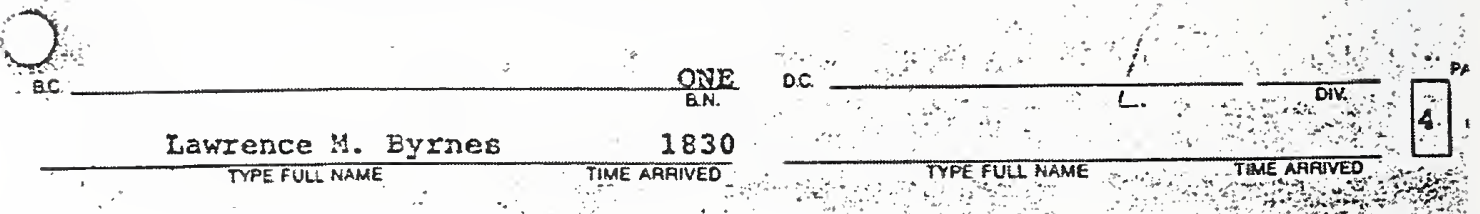


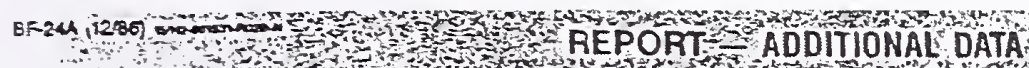

- STRUCTURAL FIRE TRANSPORIATION FIRE NON STRUCTURAL FIRE OR EMERGENCY
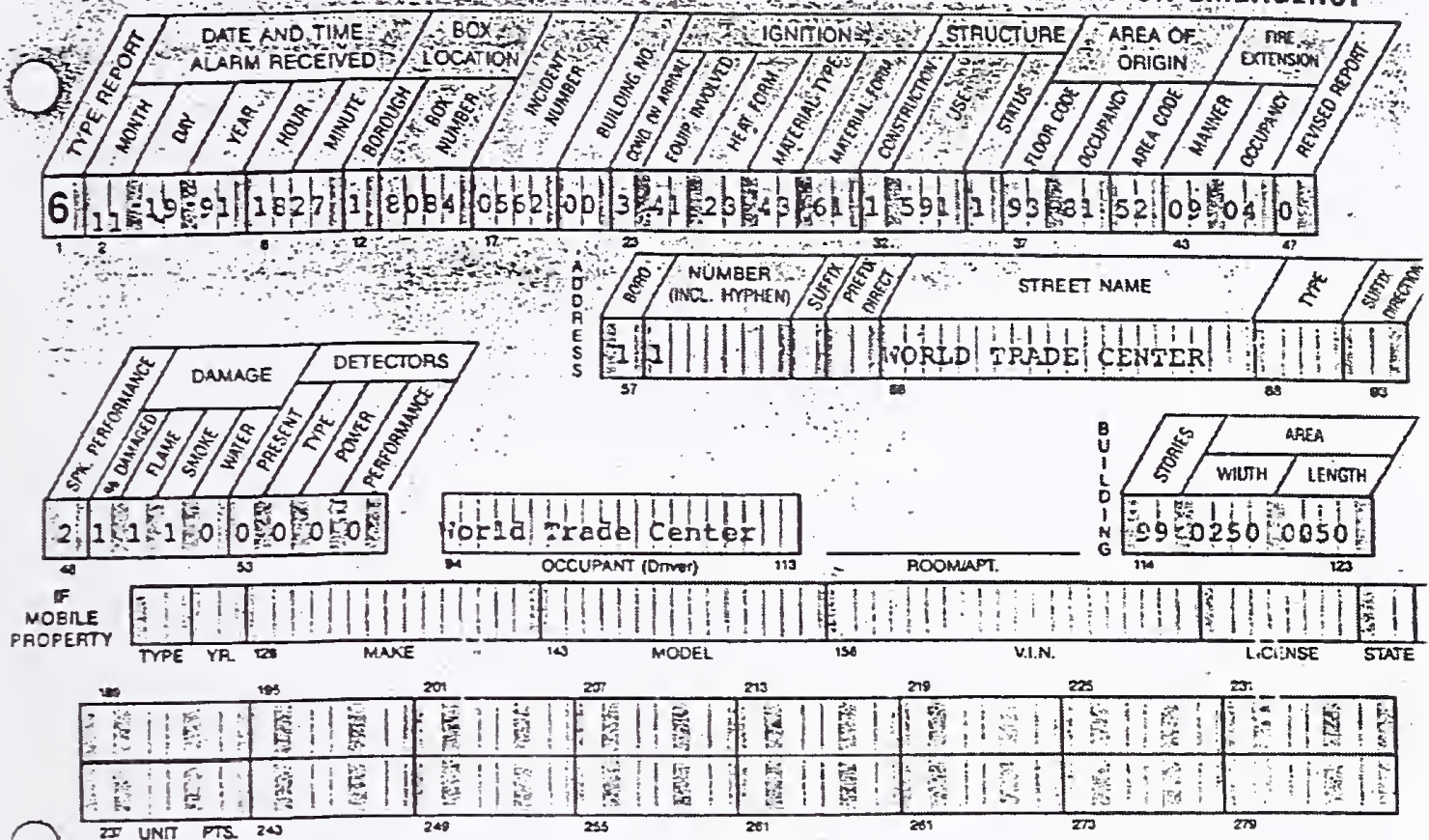

$\longrightarrow$

Iime Soguences:
Sicnal
Eox 5084
$10-84$
$10-76$
$10-44$
$10-81$
$10-75$
$22-5084$
$10-54$
UC/ 1084

mine
1827
1830
1833
1842
1845
1848
1858
1904
2006

OPERATIONS

Injuries:

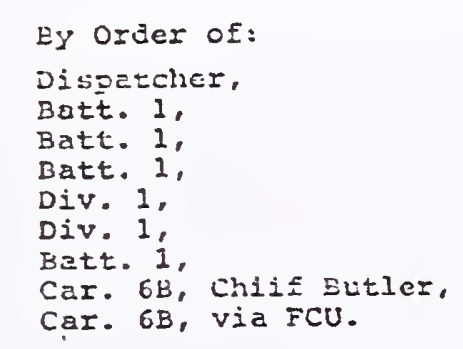

superficial burno,

chest pains.

Crit Cltations to ge sibnitted for Ladier Co. 10 and Engine Co. 10.
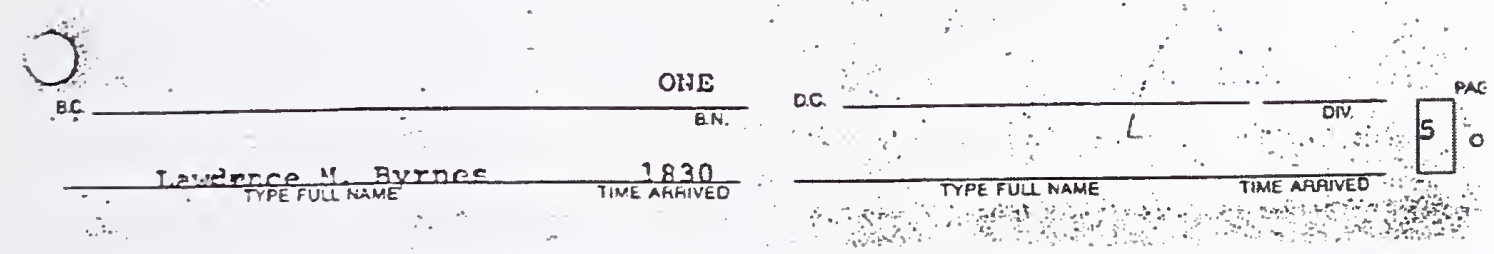


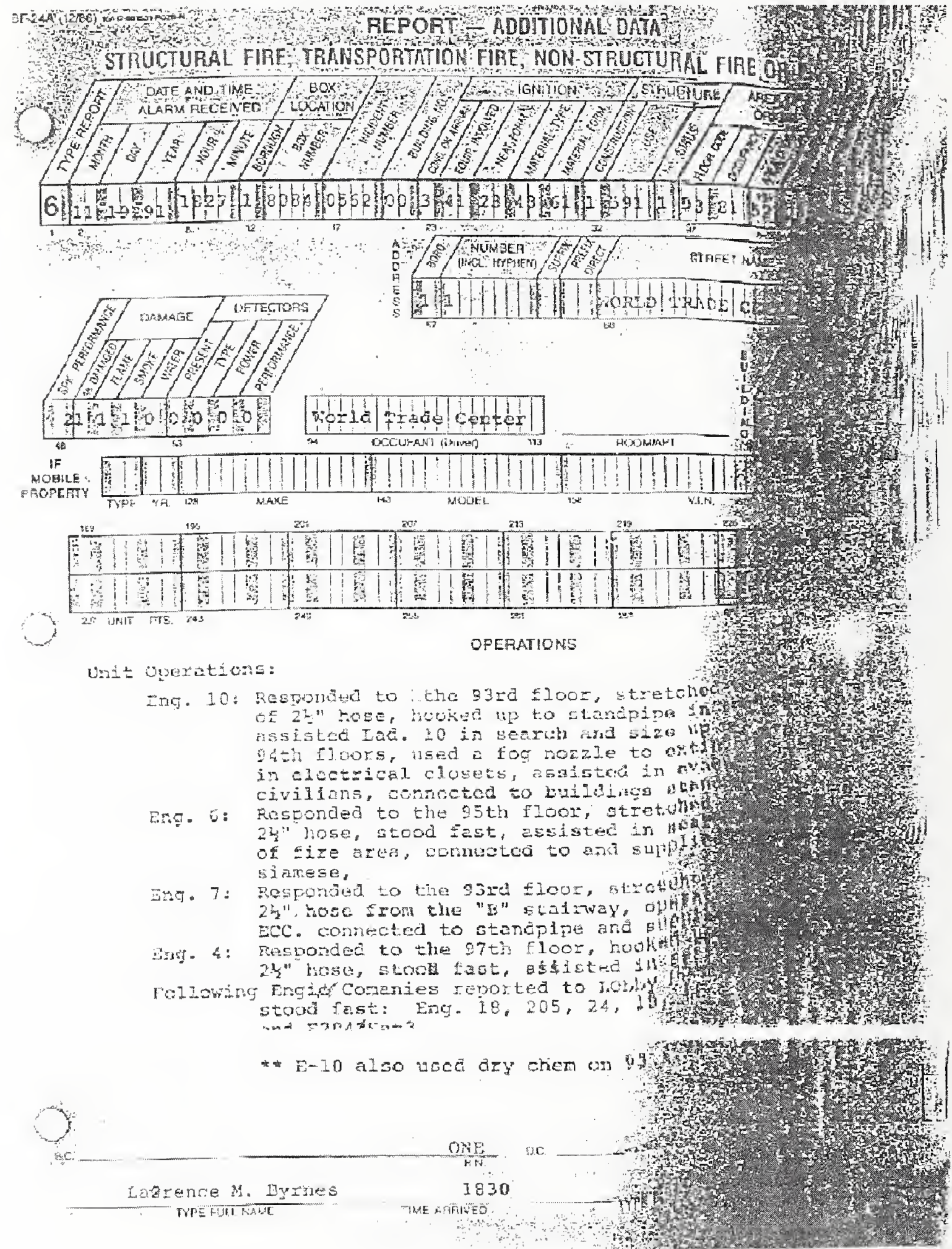



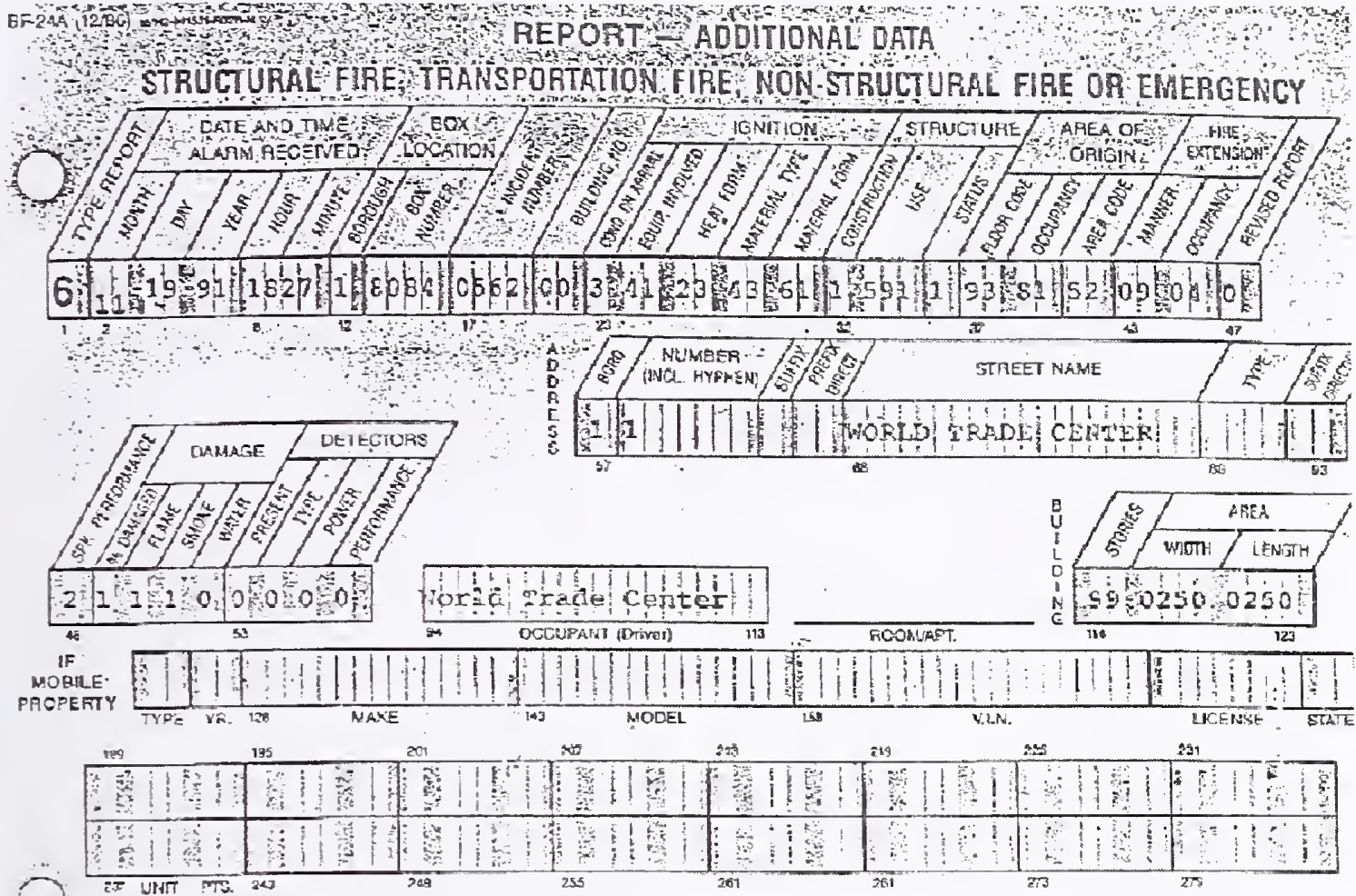

OPERATIONS

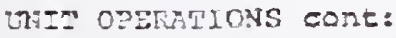

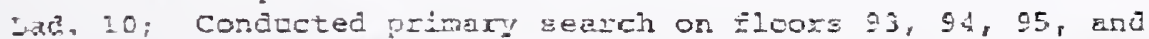

sê. and eq. Unit spit into 3 tedes, locatedafiro condition in electrical distribution ciosets, calied for placerent of hand lines, concucted opening up uperations on 1 th sioor to check for flre crtension, cvacuated unknown menber of civilians fron floozs 94 through 95 suring snitial operations,

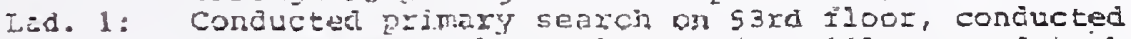
ceconess. sosrch or floors 94 to 101 , proved to be negative, conducted survey on Eloora 81 through 92 EII water danage to elscticical closets due to Hindline use, regative.

Lad. 15: Conductad primary and eccosary stectied af floors 93 to 97 , nogutive results.

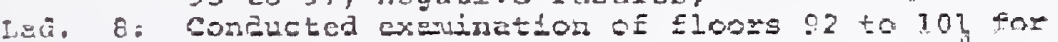

pousibie Fire extcrision fron elcctrical closets,

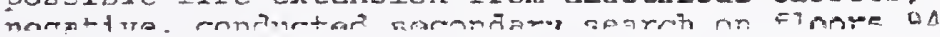

and 95 ,

Led.220: Pareorned efcondary search oi floorg 96 through 110; negative results.
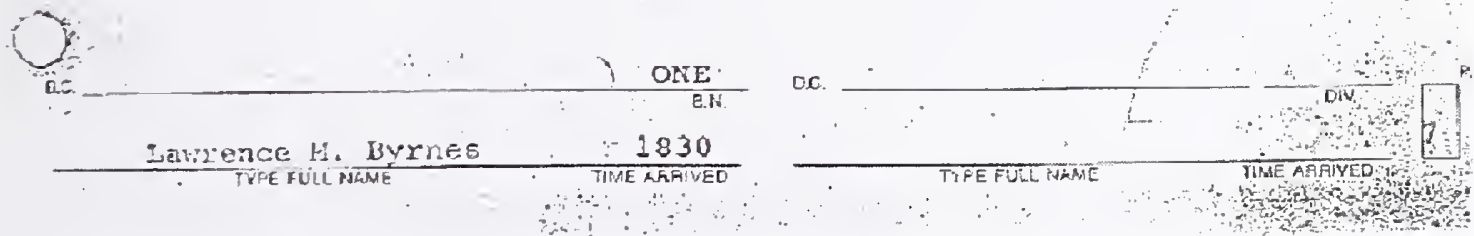

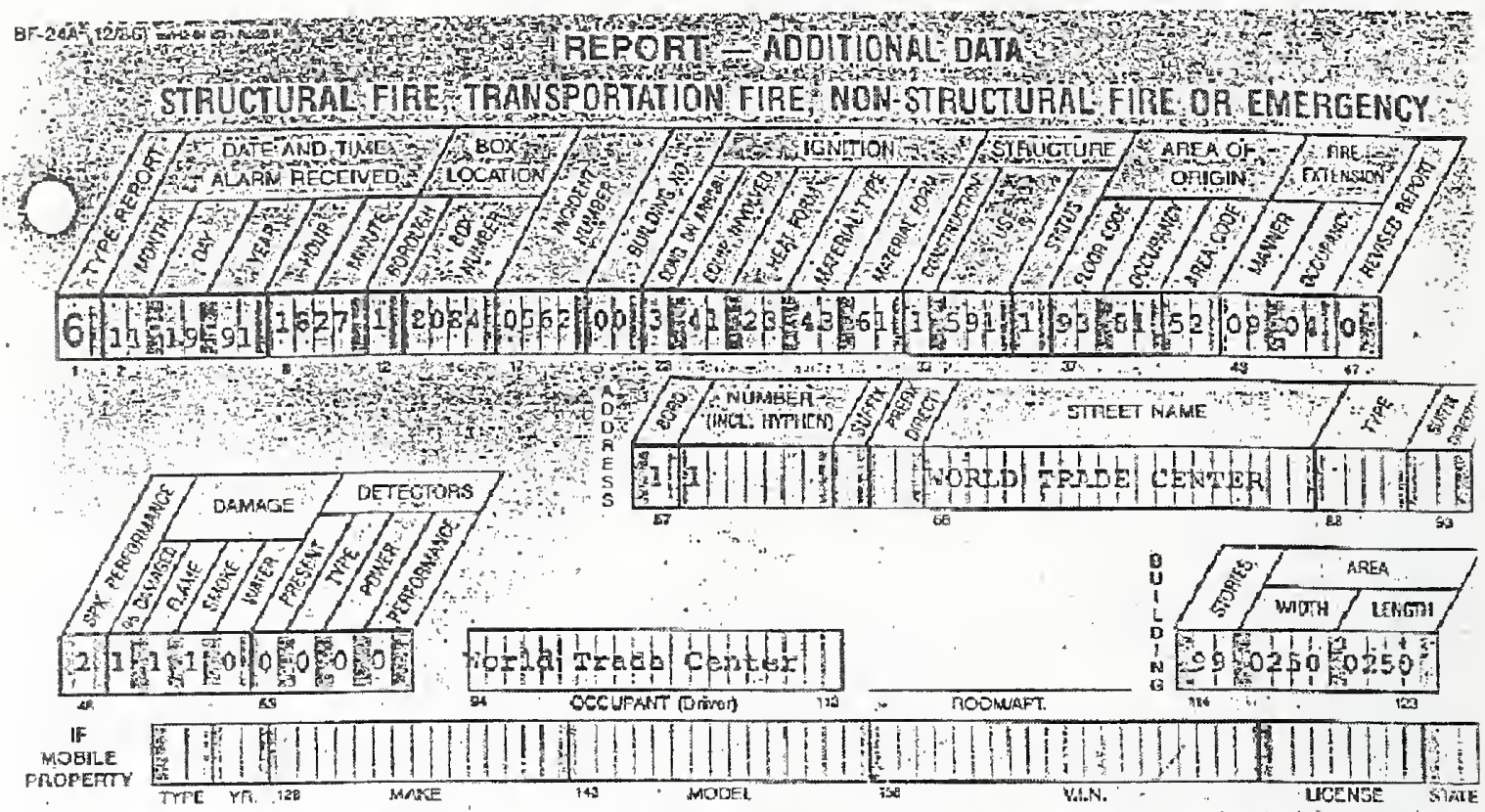

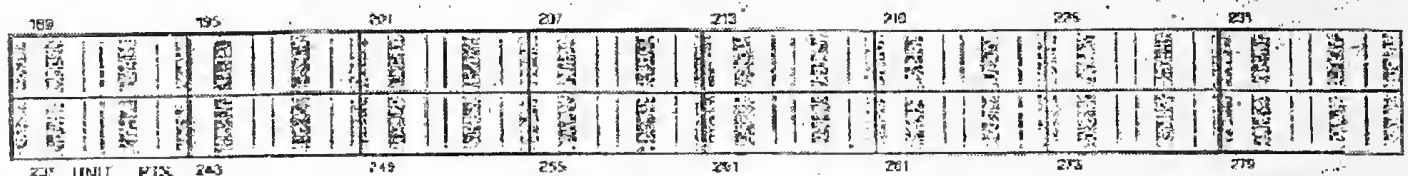

OPERATIONS

Unit operations cont:

Ead. 6: Conducted semoneary search 60 \$loors 105 to 110 , ncgotivefegultz, Fenoved (2) civilians fron stalled

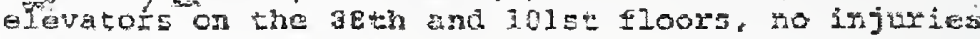
to civilians,or danege to buliding.

Lä. 20: Conducted seconary seazch of floors 90,99, and 100 neqative resints.

wa. 131: Conducted search of floors 105 to 110, with neget1ve ersulte.

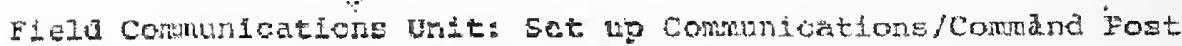

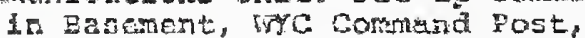

Eattalion 2 supervised I.obby staging Area,

3uttalfors 4, 6, ma 7 pparated on various floors gith undts so assigned.

Division 3 operated es Operations OTC on yaxious Eioors. Divion 1 operated at the Command Post as cic.

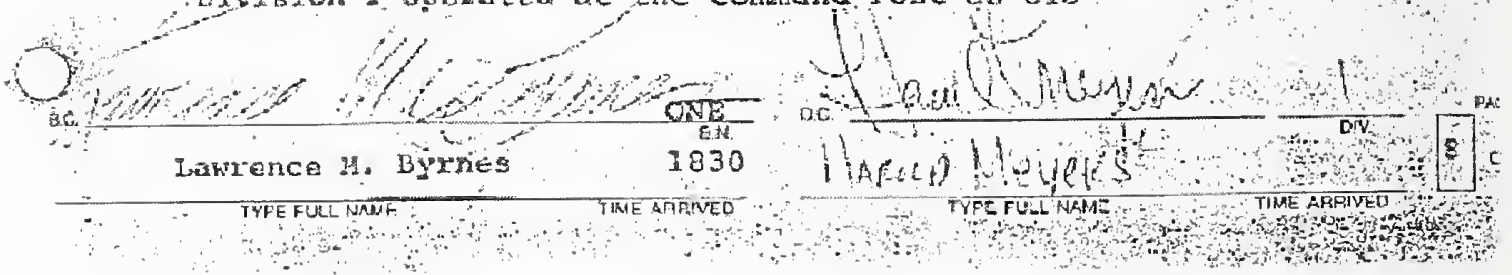




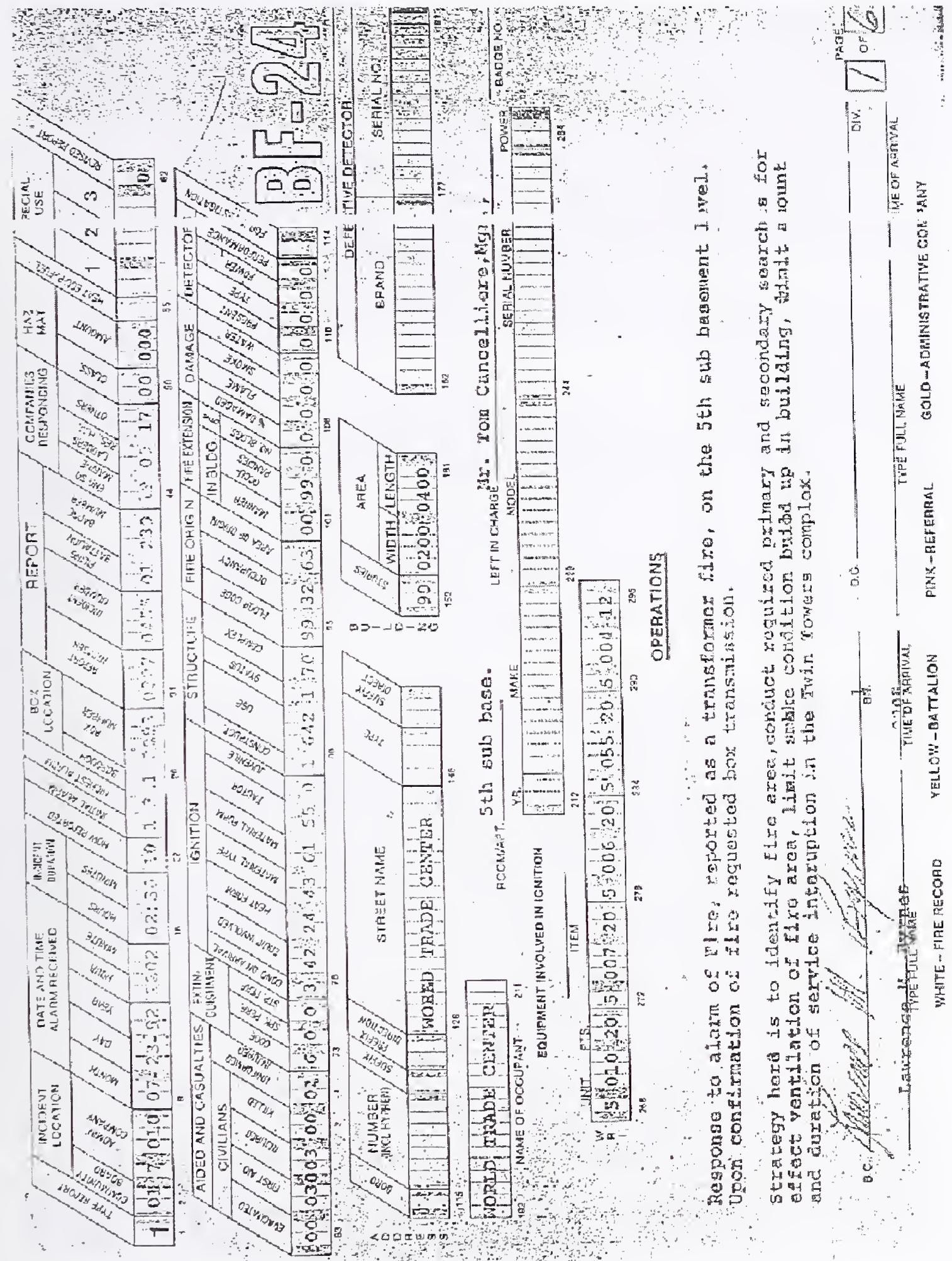




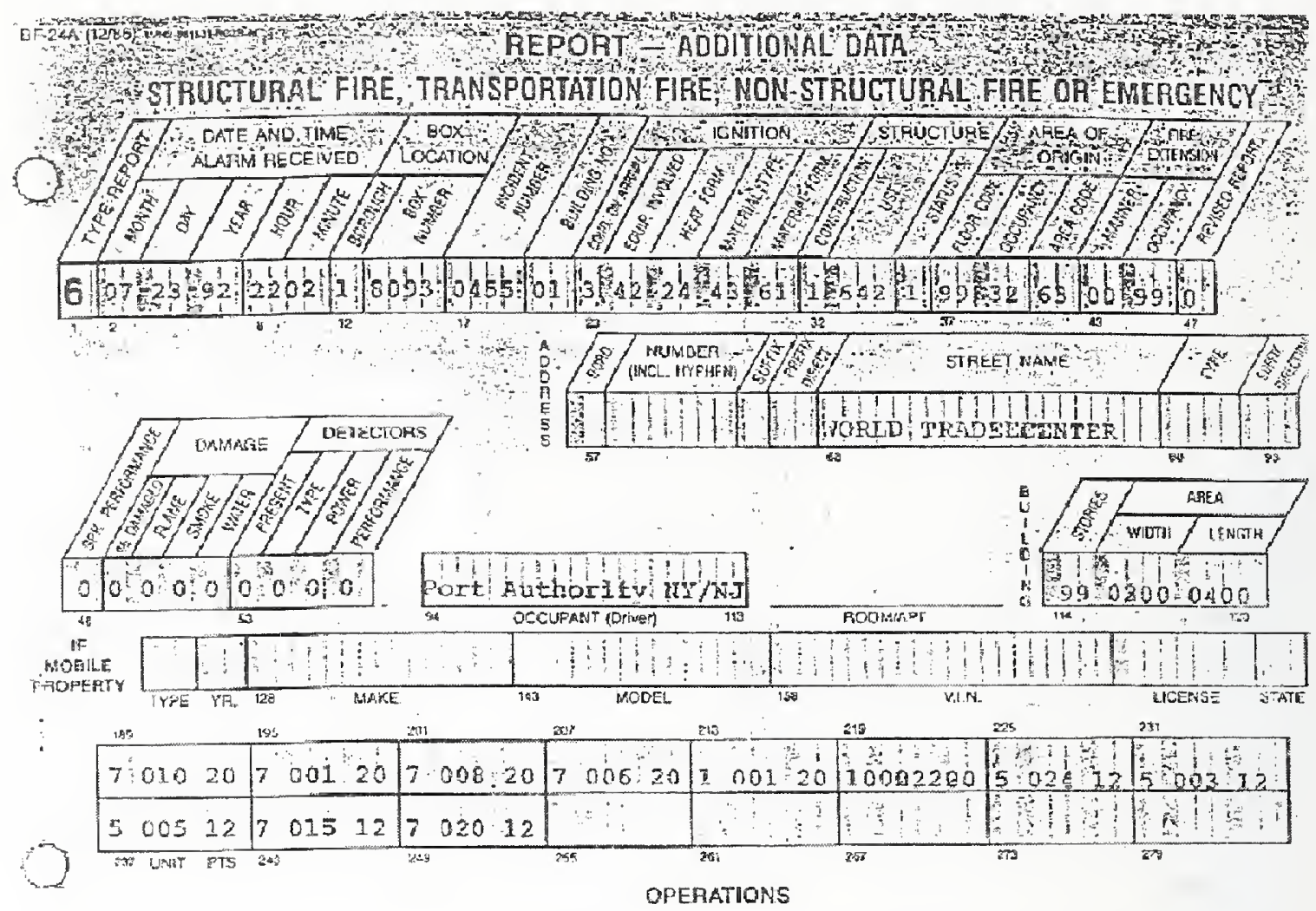

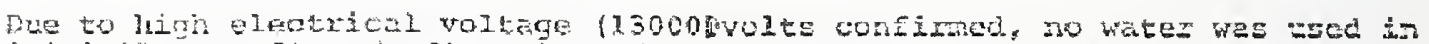

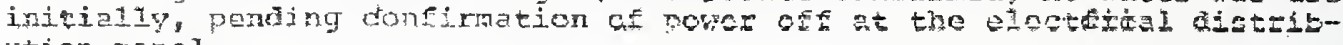
ution parcl.

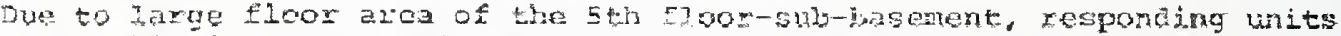

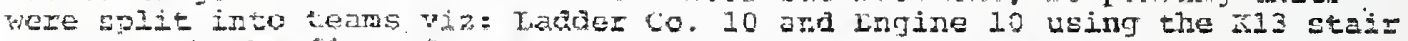

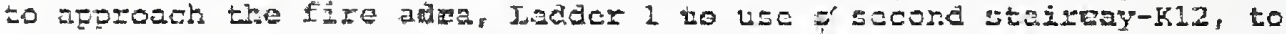

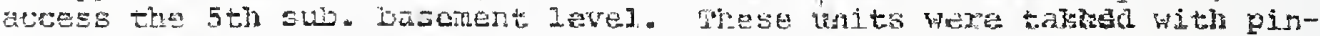

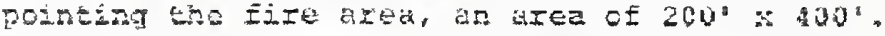

A monbez of bader co. I having roun the fire stuation in a very large pover disfitibution oanel, atchece to relay infoumation to his offjcer. Prior to his transtission fireijghter was struck by a shock blst generated bye the involved pancl. Ladder 1 firefighter jnotked minchasience reguired a comsertied effort to remove to a separabe safe area.

Unit operákions.

Engine 10 - operated on 5 th sab level, stretched a 2 s. hand line Exmom the stempipe, operstec when powar off conflnation received. Company

Engine? - operated on fire [Ioor with line off sanapipe; opergtad

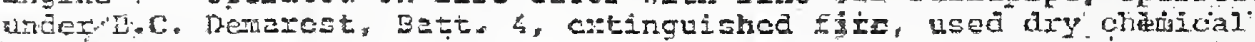

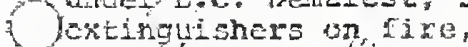

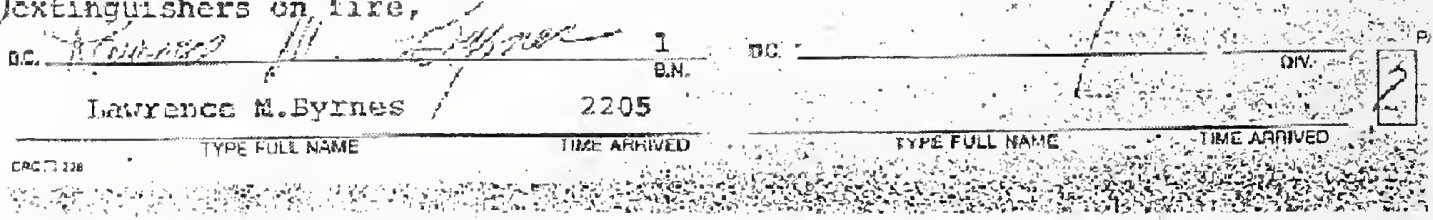



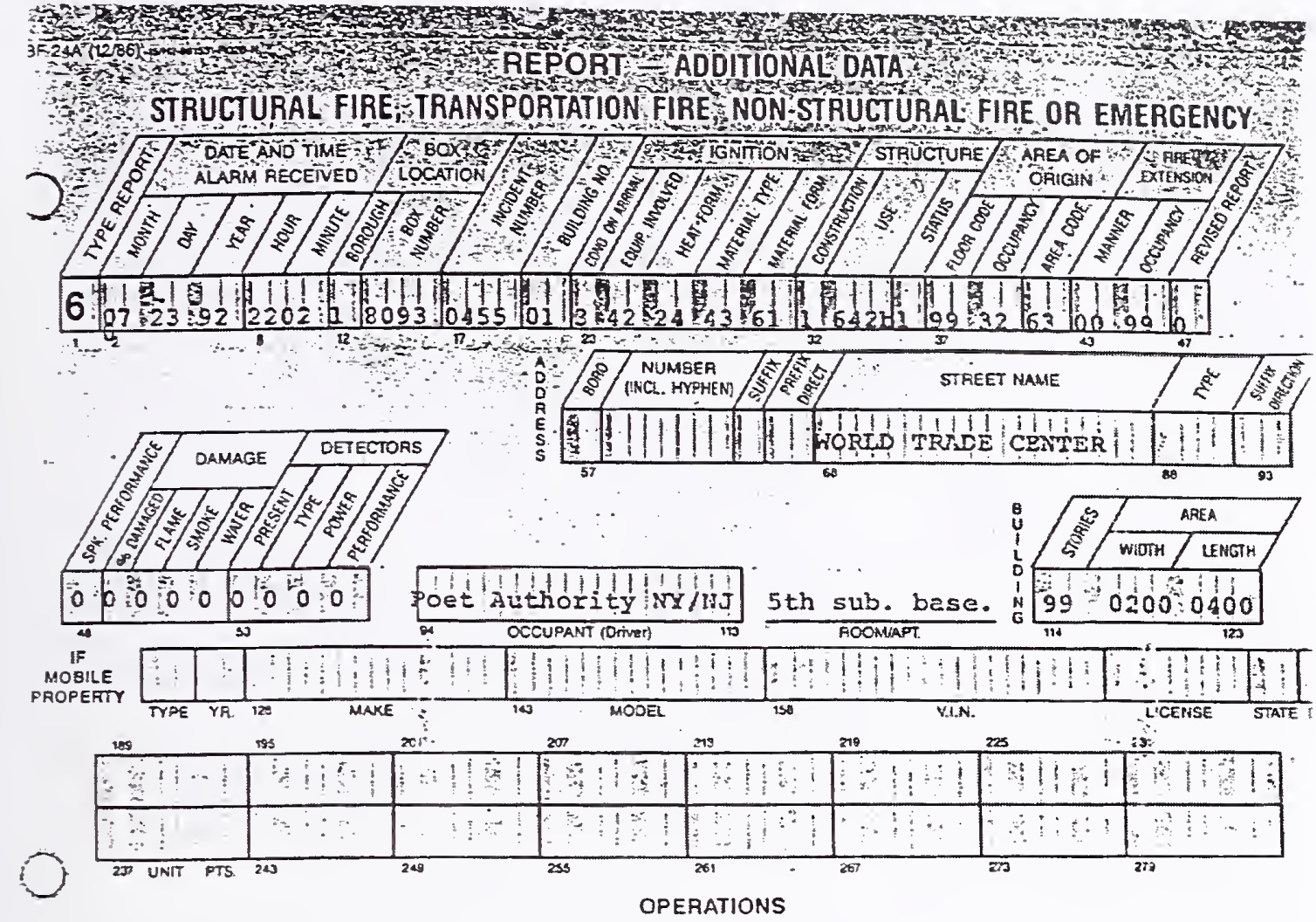

Opezation cont:

Engine 5 - Assisted Eng 7 in stretch of and operation of a $21_{i}^{\prime \prime}$ line into fire area, perfortuec search of area, overlauled as neceesary,

Engine 55 - Operated with and relieved Eng. 7 on hand line on fire floor, took lip hose lines,

Engine 4 - under supervision of BC. Turnee,B2, transported injured inember of Lad. 1 to ambulance on the El level of the fire building, relieved Ing. 10 on a Ekä Iine, cberhauled, took up hand Iine,

Engine 24 - Transported Air Cylingees fire area under supervision of BC. Jackson,

Engine 3 - Ordered to and did provide air cylingers to operatind units of the 35 level.

Engin

Stood Fast - Eng9Sat1, Engines 15, 23. 33, 34, 207/Mari, 284/Sat.3,

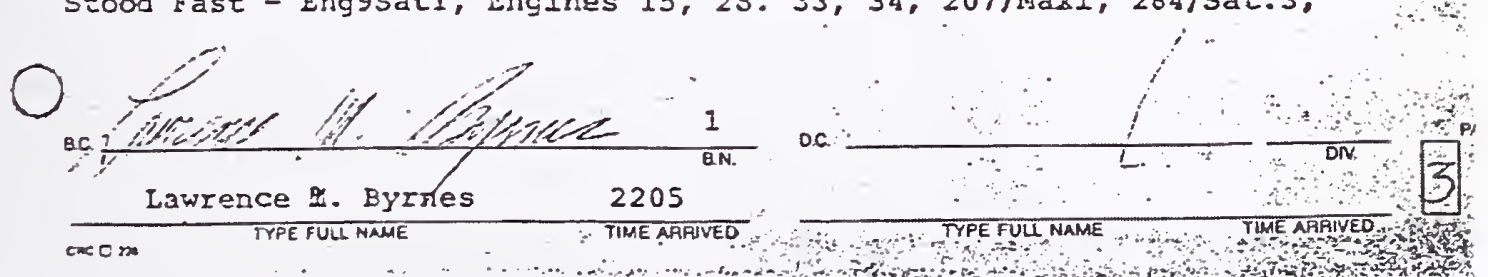




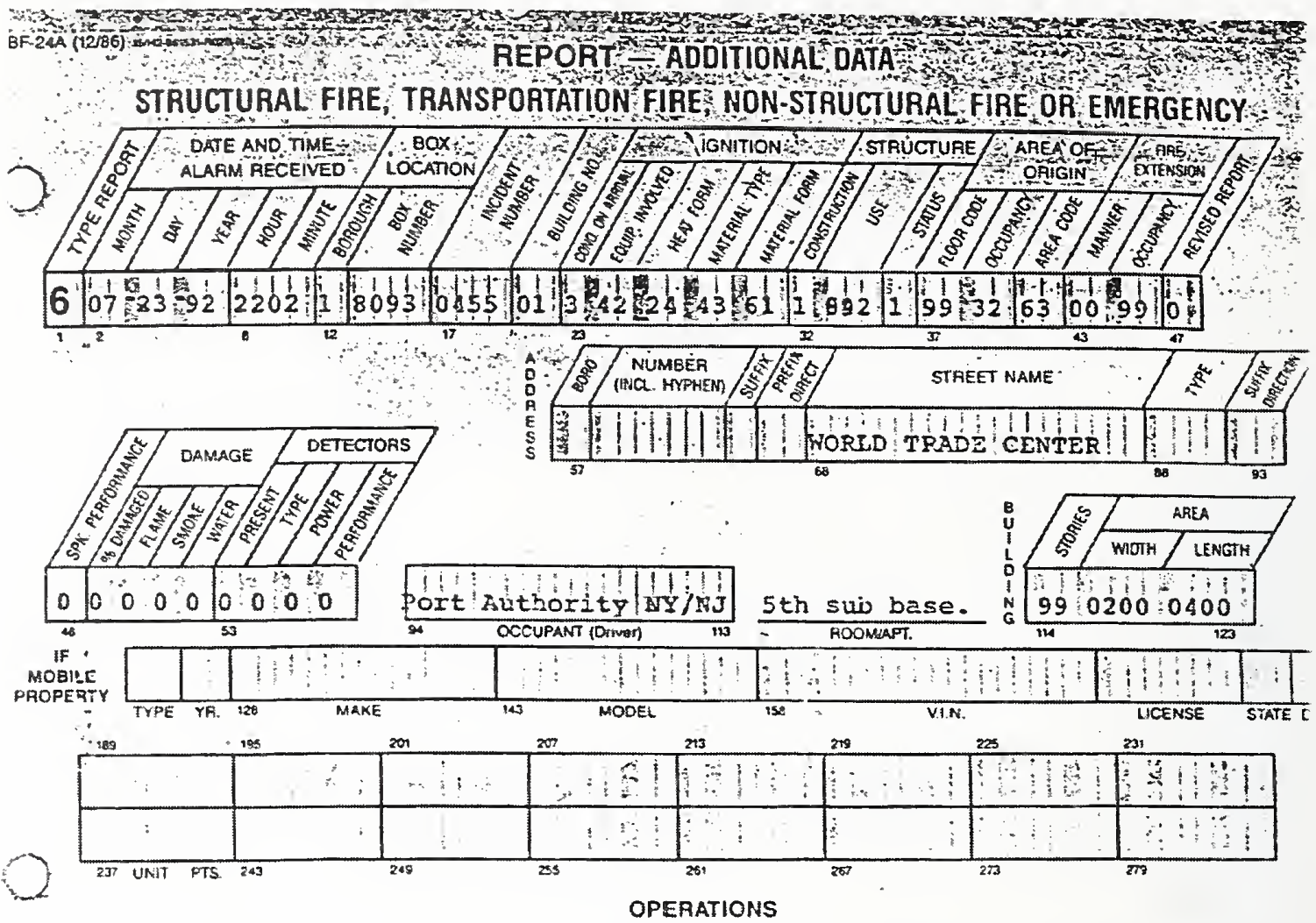

Opezations cont:

Latder 1- Operated at the 85 level, conoucted seach to pinpoint the fire area, concucted a primary search for nossible employees trapped. FE'. Anodio injured in explosion of 13000 volt distribution panel,

Lader 10- Performed a search of the E5 level 2to identify the fire area, and searched for possible trapped employees, gatherea and used dry clidnical extinguishers on the fire prior to porer removal, omerhauled as requirired,

Eadder $\varepsilon$ - Performad a seconcary search of the fire area, used dry chạnical extinguishers, assisted in overhauling,

Jsader 6- Paiced and usec portable exhaust fans in stairgells to effict ventilation, took up,

Tadior 15- Sunolied snare SCAA cylinders to stanina erea.

Lader 20- Supplied spare SCBA cvilinders to staging area,

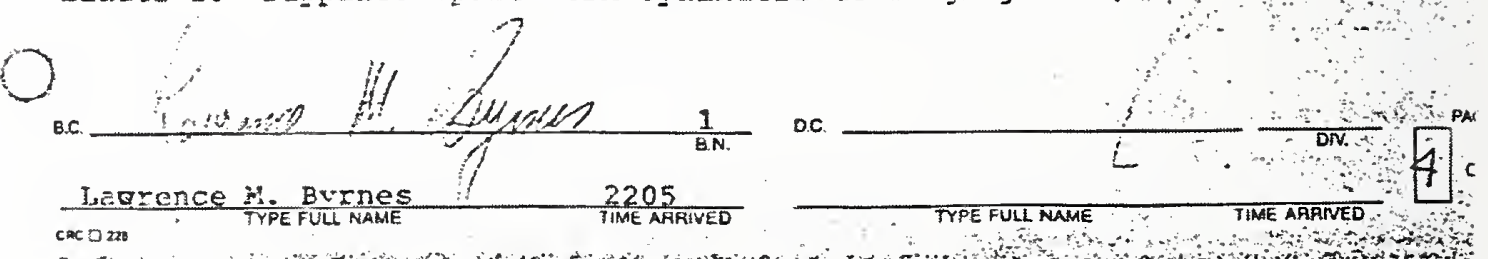

$m$ - . . 


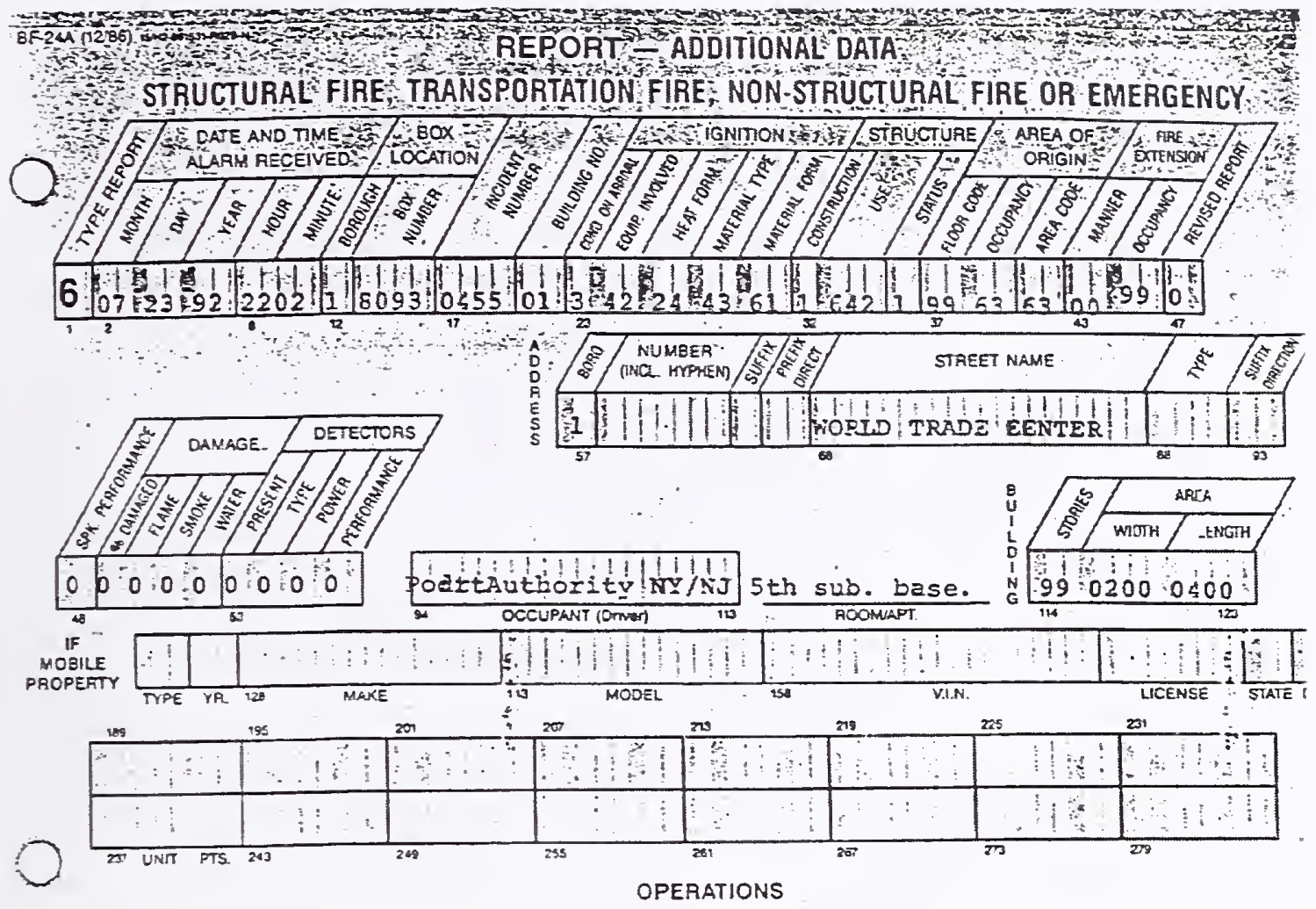

Operation cont:

Eencue i- Compary split to perform sereral ìcerations-

1. hssisted in administering êrst aia to Injuzed firefighter,

2. Conducted seconcary search cf fire arca, jegative,

3. Used Thermal Canera to chec!: For Dossible fire extension,

Rescue 2- Rssisted (2) civilian electricians (with SCDA's) to confirm power off in electricalpanel, relayed confination of power off to Comnand Post. Lssisted in hand line iperation, assisted in VES OE fire floor.

Chief officers present: DAC R. Palrer, Cir Duty DC. P. Kanson Div. 1, R.C. I. Byrnes Batt. I, B.C. W. Demarest Batt. 4, B.C. Costa Batt. 7, 3.C. Miccio Batt. 6, B.C. R.Turner, Batt. 2, B.C. Harâne Rett. 9. -... unsuval bur. دo,

山.L. russ Ecite. It. 


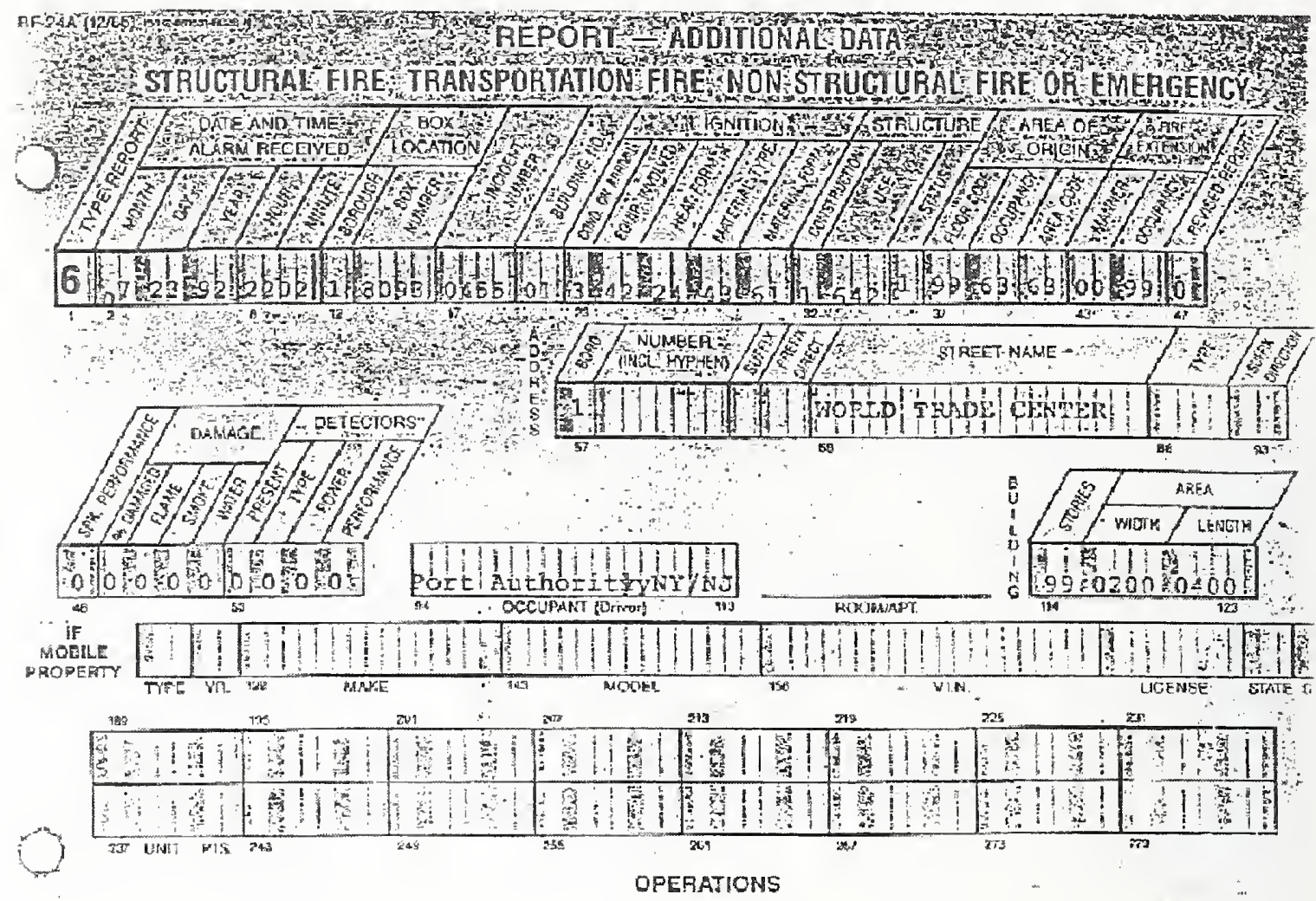

Ogerstions cout:

Greate alame transujtted aur to hagnitule of fire area imvolved, potential for smbje and heat problews, muluers of employes in building reed tosurply and relieve oporating personse.

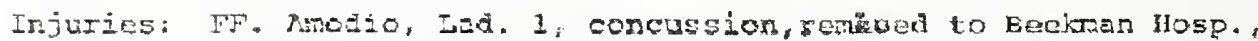

FF, Cancel, Lad 10, granted Nr.,

PF. Eansor, Lad.jo, granted Mr.,

FI. Selletti, Itad. 10, Granted in.

Note: Staff and aployees of the New York Fort Authority provided excellent quidance and assistance. Help proviod included mechanical, ventilation, electrical diotwivution disciplines which rabe fur a ruch eesier fire ground operationg

(t)

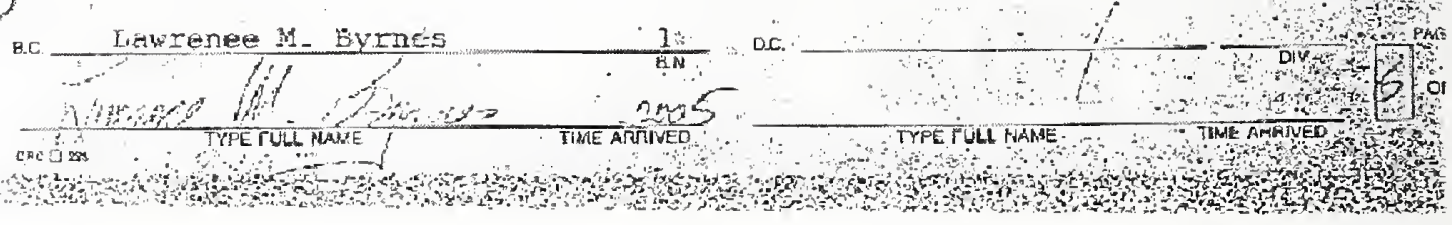




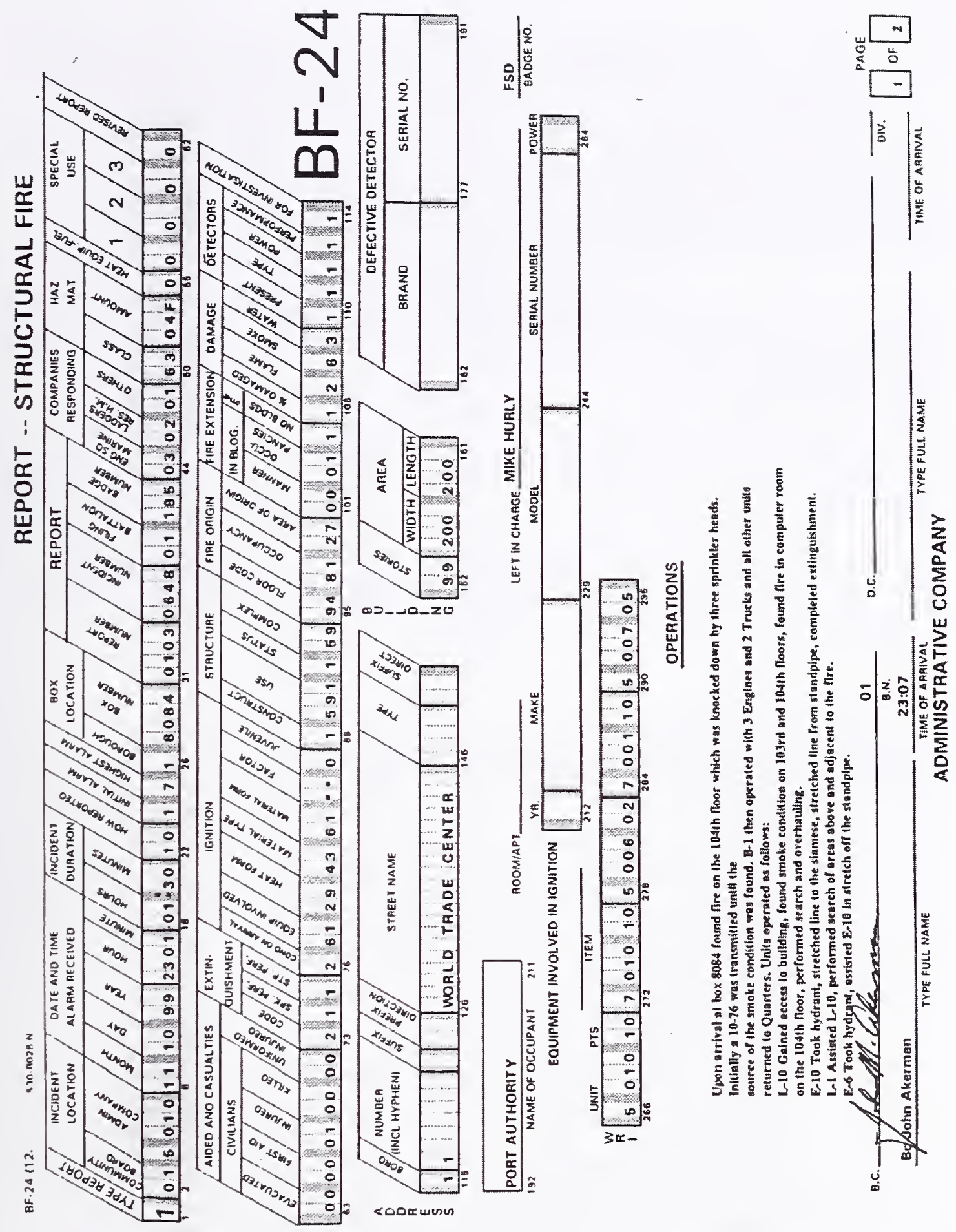


REPORT -- ADDITIONAL DATA

STRUCTURAL FIRE, TRANSPORTATION FIRE, NON-STRUCTURAL FIRE OR EMERGENCY
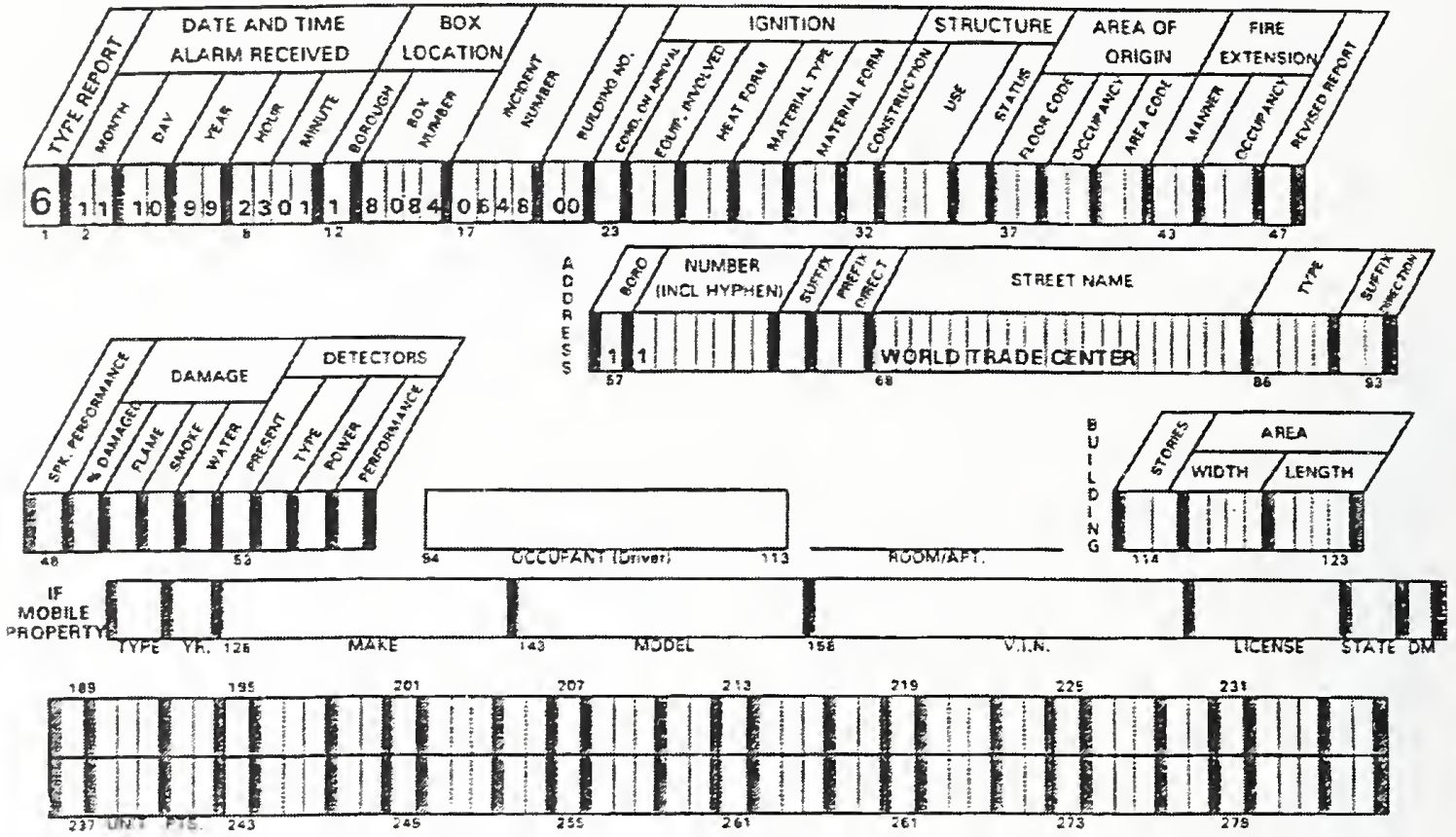

OPERATIONS

E-7 Stretched precautionary line from sand pipe.

F.S.D. for World Trade Ceater - Mr, Mike Hurb

O.E.M. L. Wilson.

F.M. Kueglet \$361 respooded on BF's ona kaskledge of prior suspacious activity. Jab M11201.
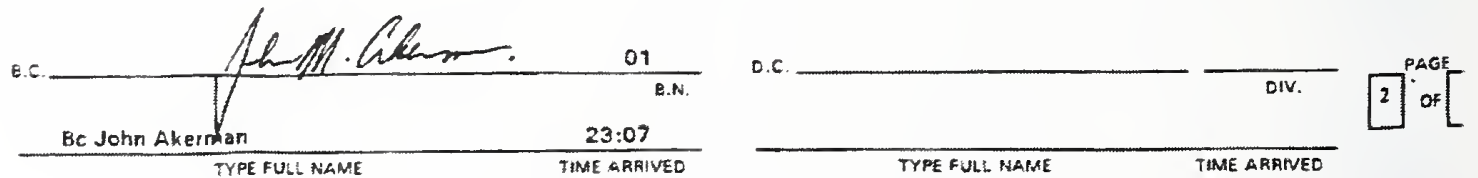

TYPE FULL NAME

TIME ARAREO 


\section{Appendix E \\ SIGNIFICANT FIRES IN WTC 2}

Significant fire incidents occurring in WTC 2

\begin{tabular}{|c|c|l|c|c|c|c|}
\hline $\begin{array}{c}\text { Significant } \\
\text { Fire }\end{array}$ & $\begin{array}{c}\text { Incident } \\
\text { Date }\end{array}$ & Fire Location & $\begin{array}{c}\text { Sprinklers } \\
\text { Activated }\end{array}$ & $\begin{array}{c}\text { \# Standpipes } \\
\text { Activated }\end{array}$ & $\begin{array}{c}\text { Cause of } \\
\text { Fire }\end{array}$ & $\begin{array}{c}\text { Material } \\
\text { Ignited }\end{array}$ \\
\hline 1 & $5 / 19 / 75$ & Floor 32 & - & 3 & Incendiary & Trash/waste \\
\hline 2 & $4 / 12 / 77$ & $\begin{array}{l}\text { Duct work over } \\
\text { grill in restaurant } \\
\text { on floor 107 }\end{array}$ & 2 & & None listed & Duct work \\
\hline 3 & $3 / 22 / 93$ & $\begin{array}{l}\text { Fan motor room } \\
\text { on floor 108 }\end{array}$ & 2 & & $\begin{array}{l}\text { Mechanical } \\
\text { failure }\end{array}$ & Not classified \\
\hline
\end{tabular}




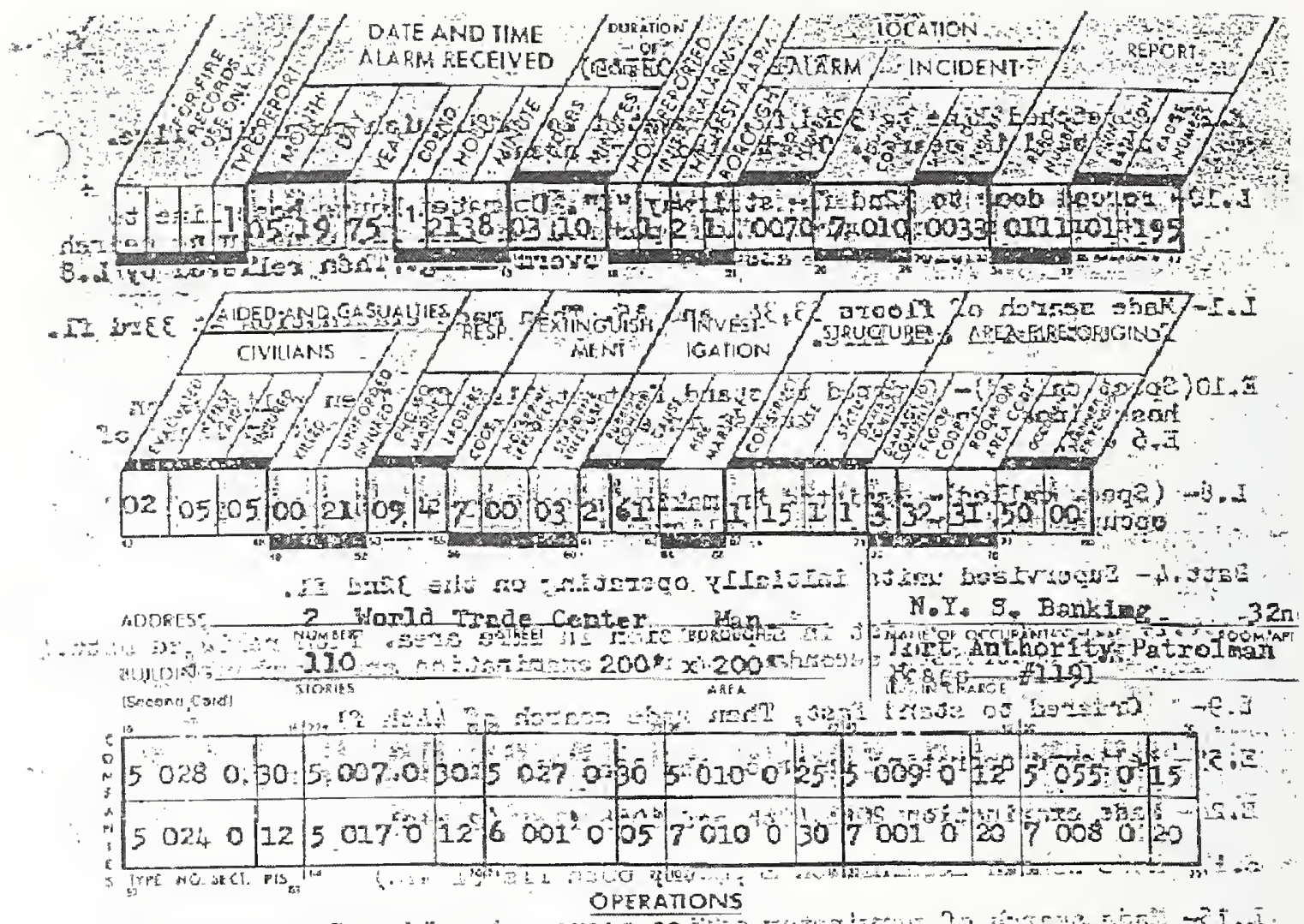

Jjon arrota

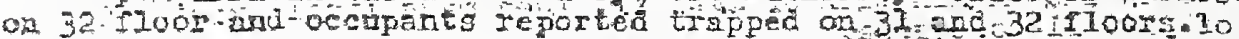

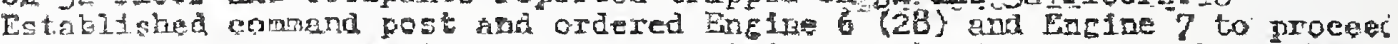

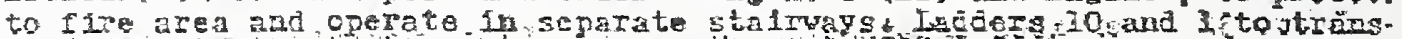

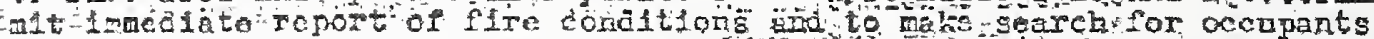

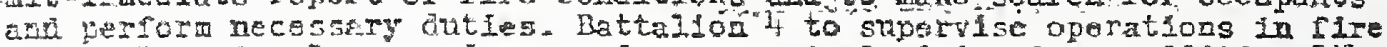

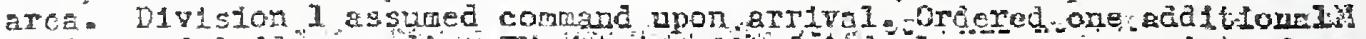

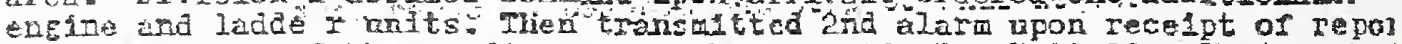

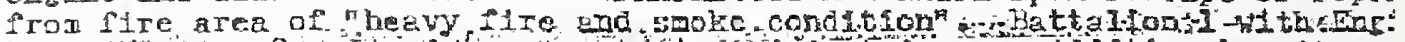

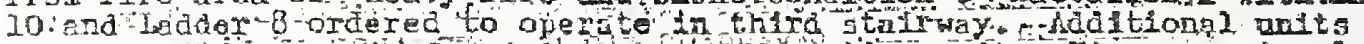

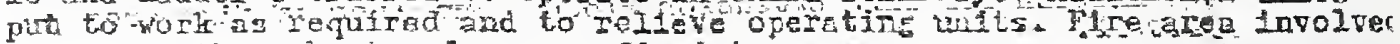
core troa throuthout eno was confined to samb.

Four olnor f1ros in previous two mours were suspected ersoging were

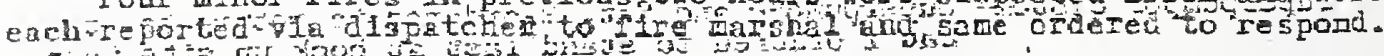

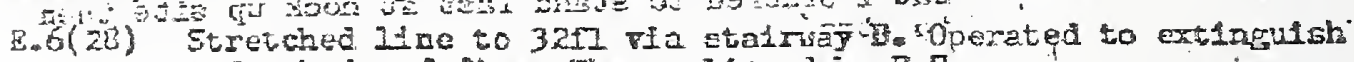

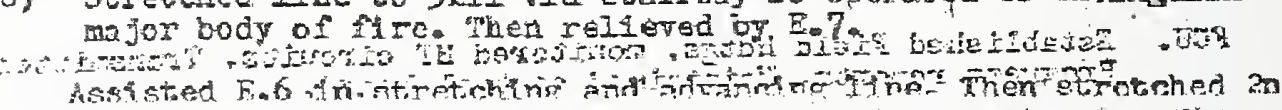

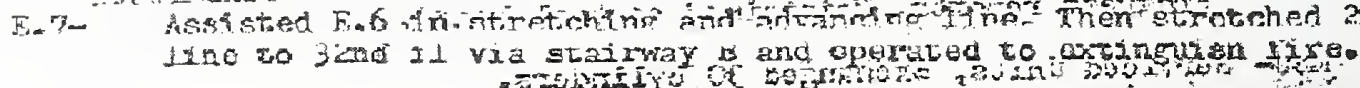

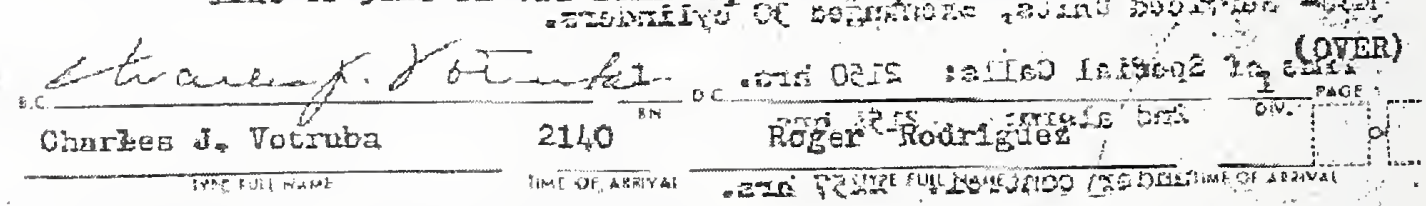

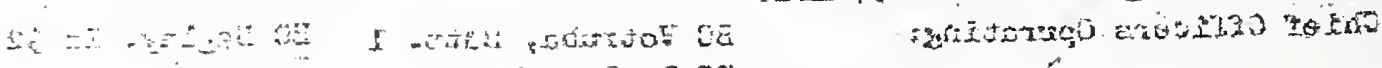

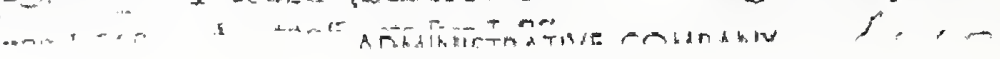




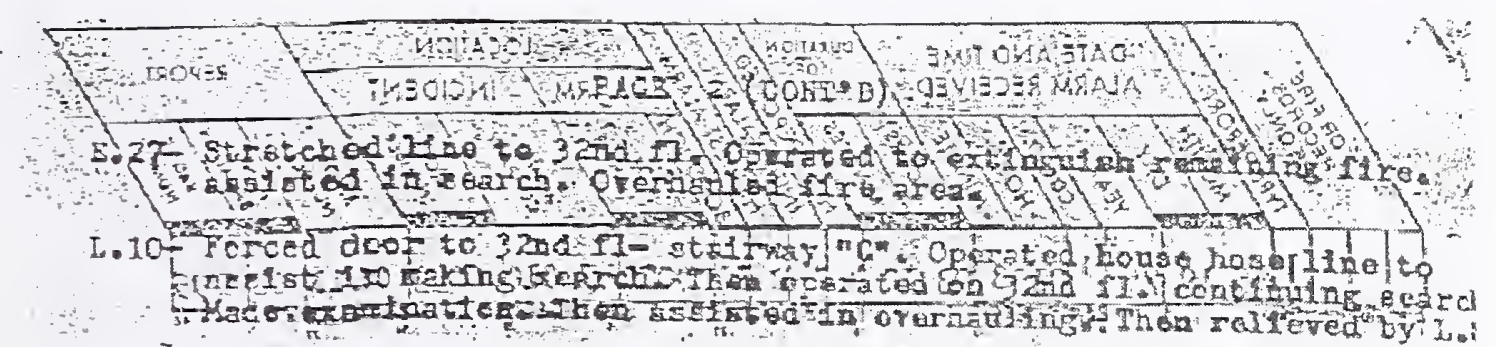

L. Tro

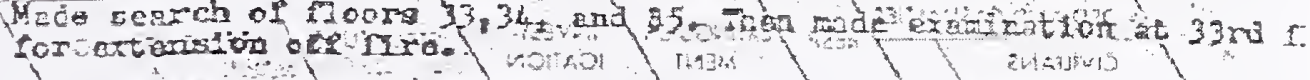

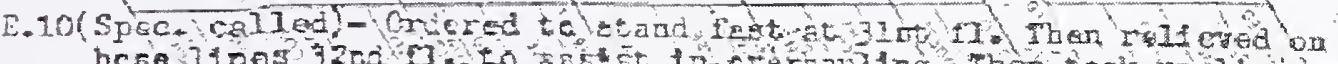

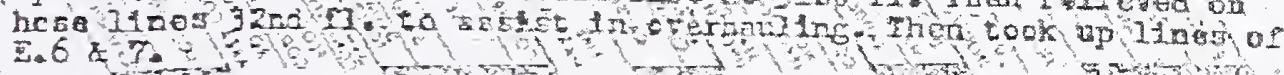

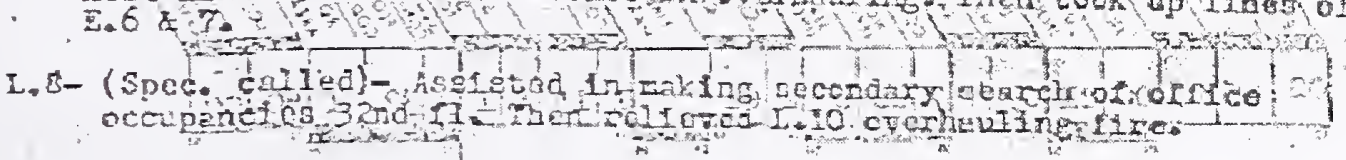

Bavt.4- Sugervised und to fratally operating on the 3and fl.

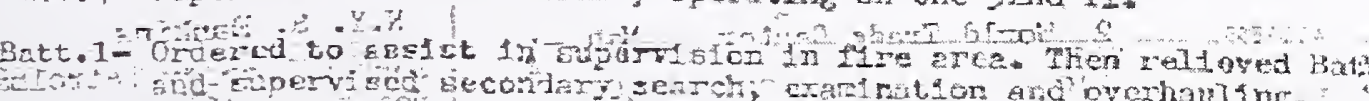

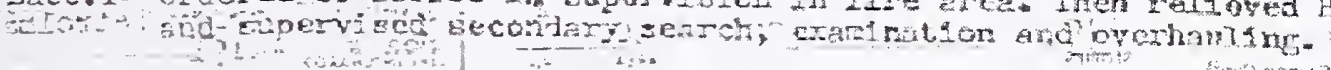

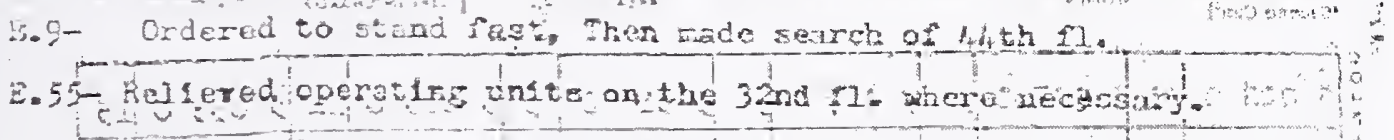

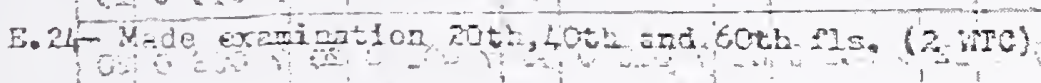

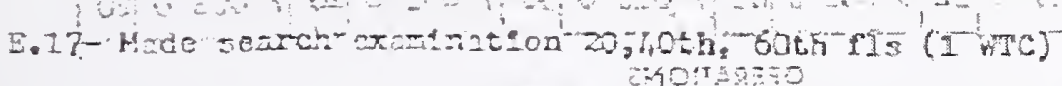

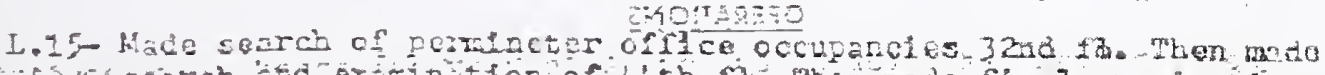

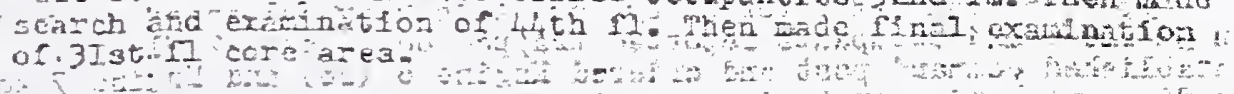

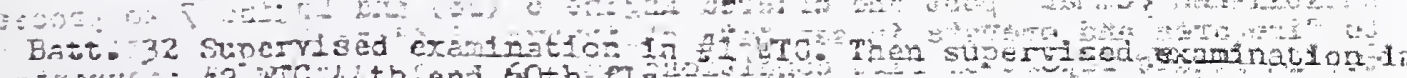

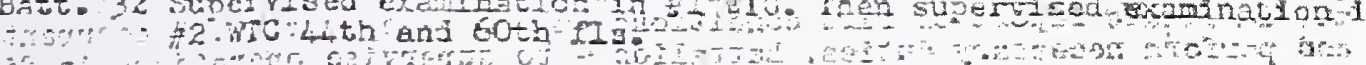

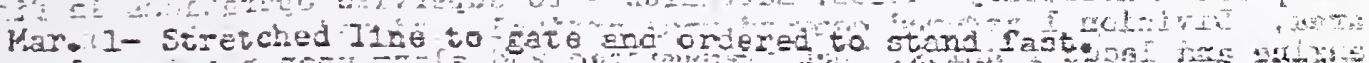

-

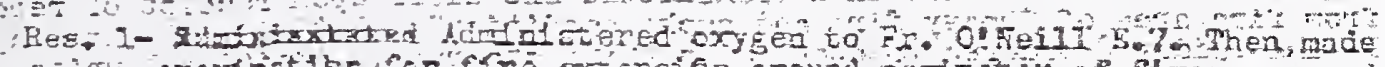

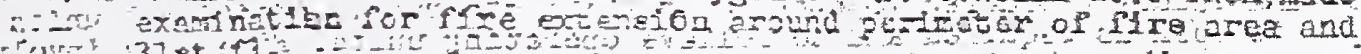

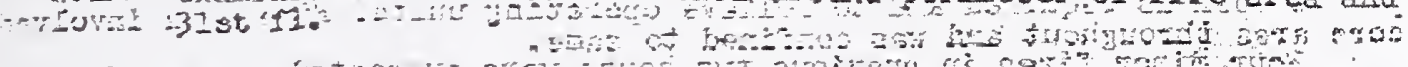

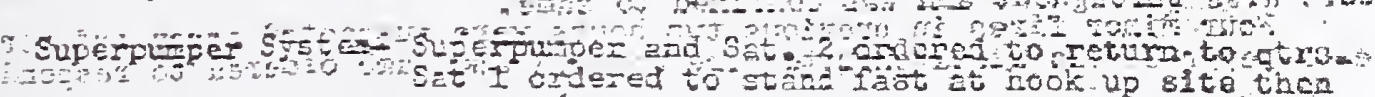

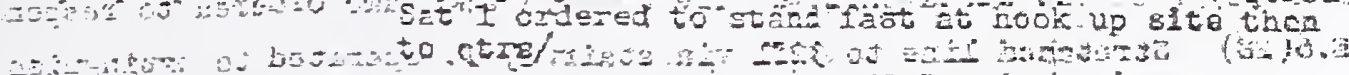

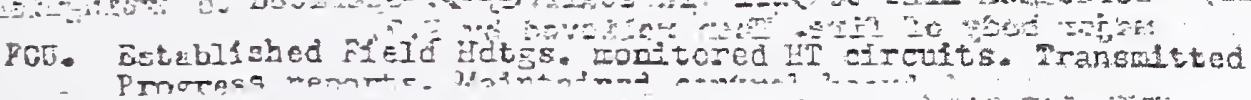

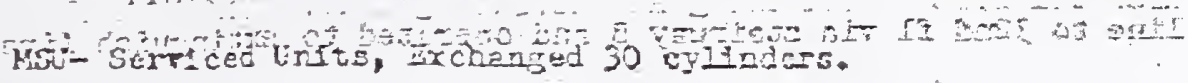

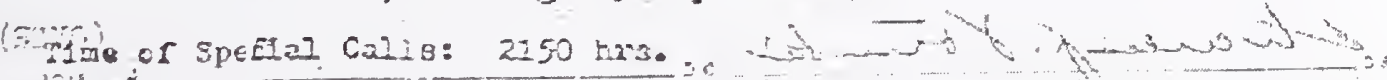

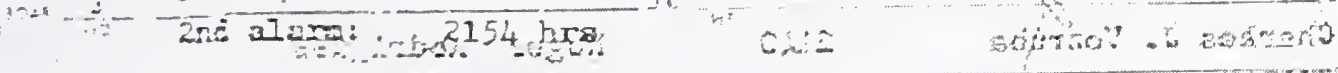

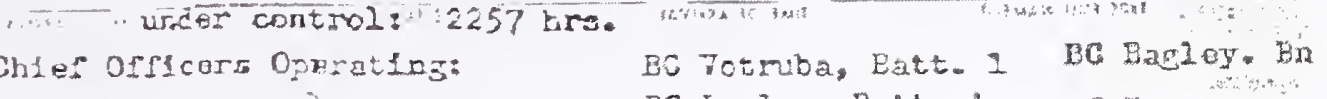




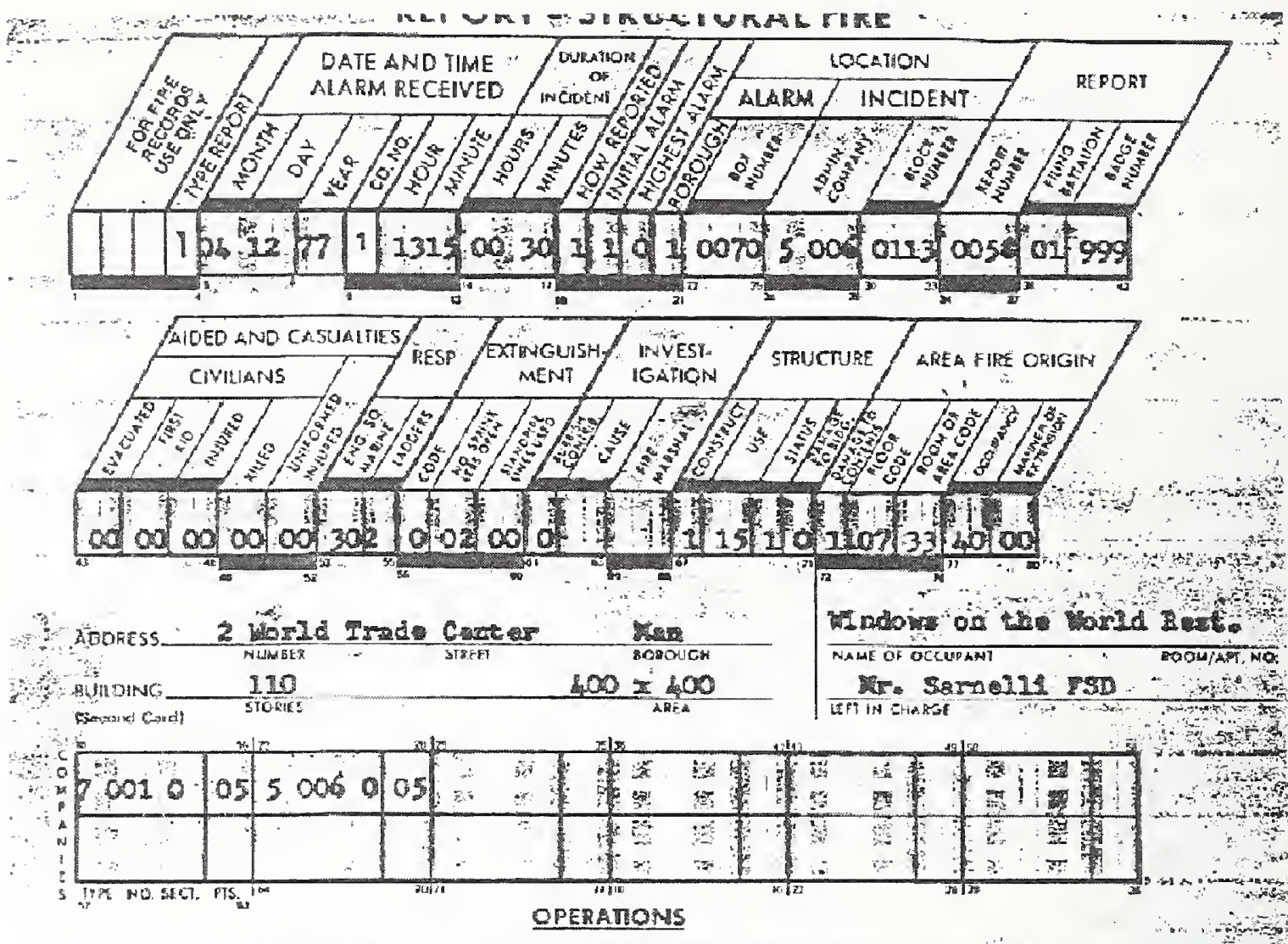

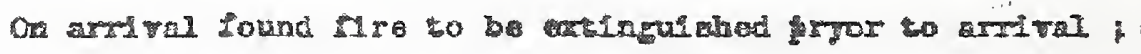

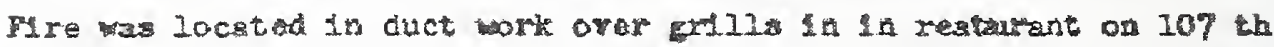

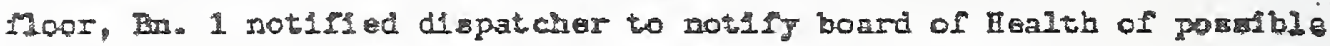

food confardation fron bost wok and cares in restaurant.

B. 6 Took rolled ups to 107 floor, atret ched Ilst and atood fasto

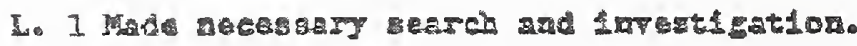

8.2. 2 ca reene replaced two spraklar heads.

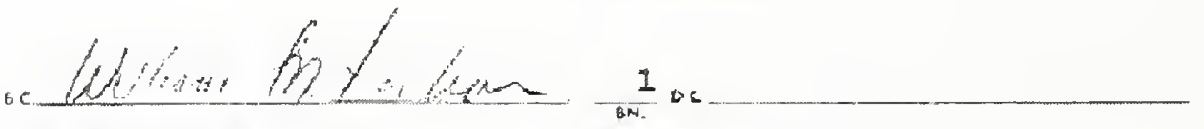




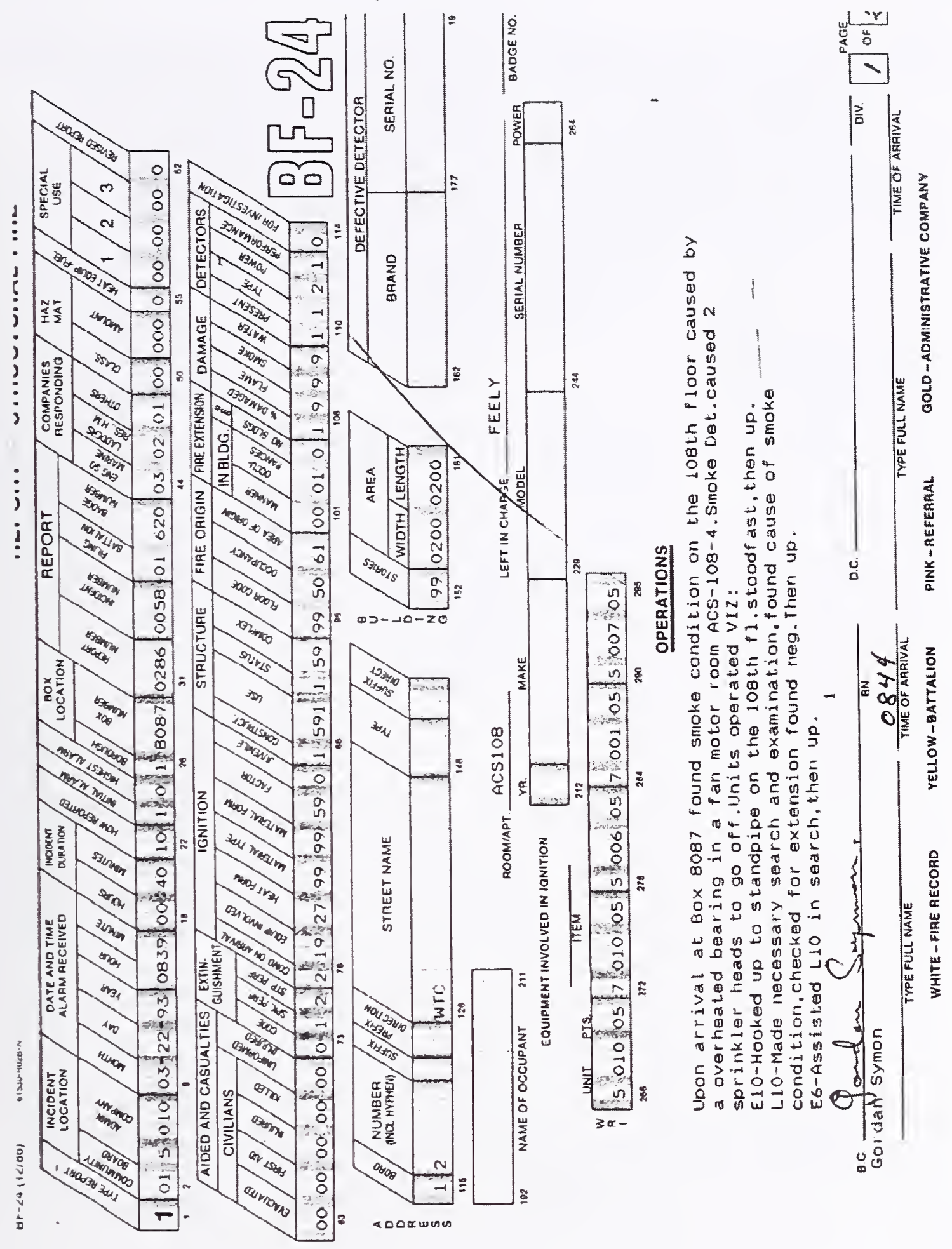


REPORT - ADDITIONAL DATA

STRUCTURAL FIRE, TRANSPORTATION FIRE, NON-STRUCTURAL FIRE OR EMERGENCY
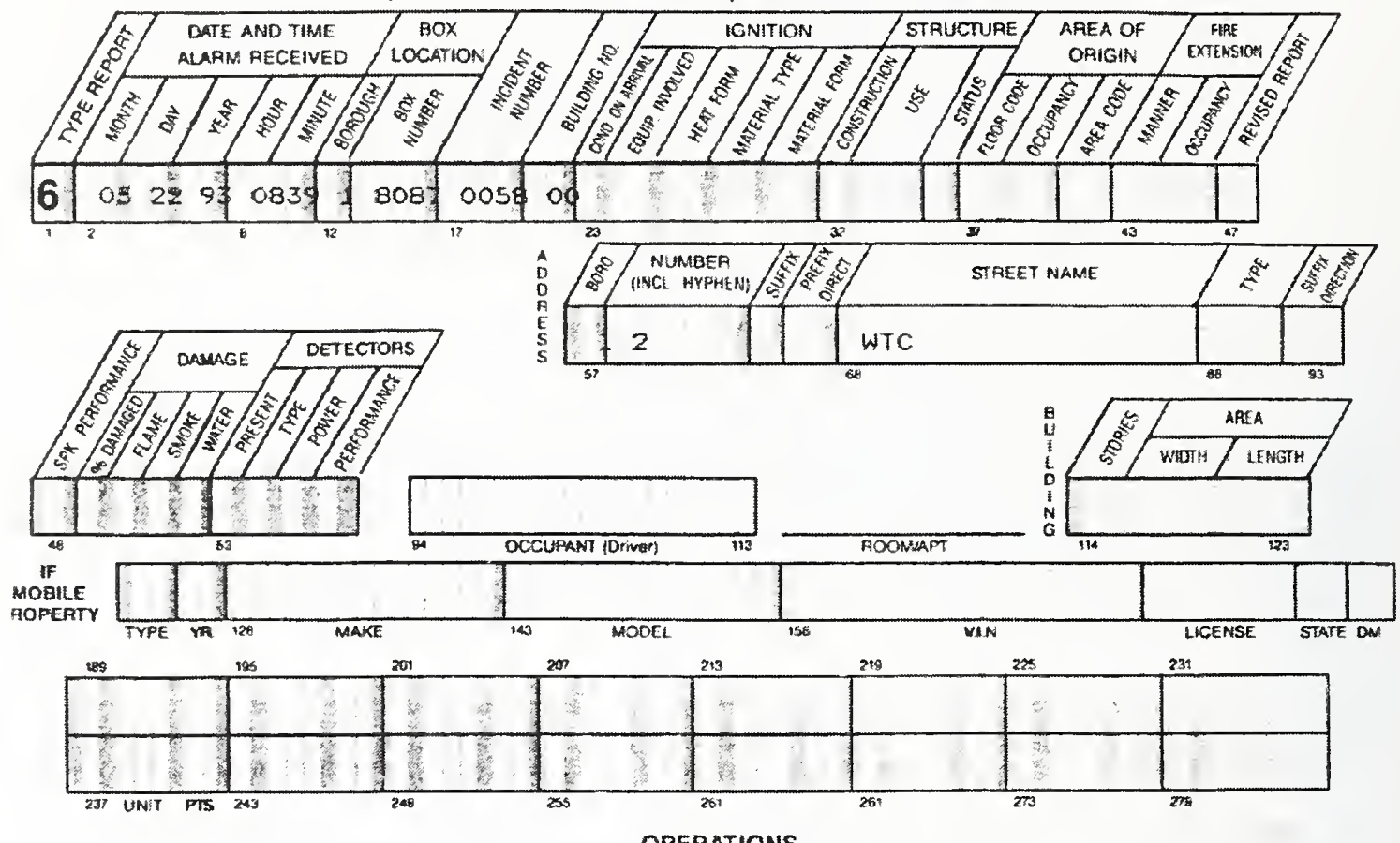

OPERATIONS

E7-Assisted ElO in stretching line, then up.

L1-Assisted LlO in search, then up.

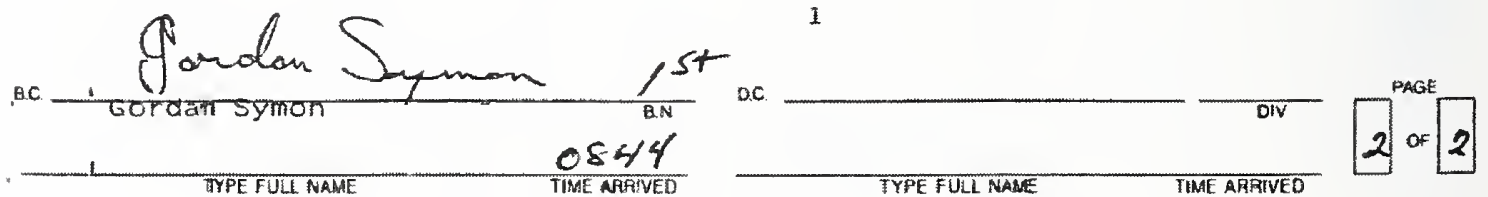




\section{Appendix F \\ FIRES WITH STANDPIPES AND SPRINKLERS IN WTC 1 AND WTC 2}

Additional fire incidents involving the deployment of standpipe lines in WTC 1 and WTC 2:

- Fires involving the use of one standpipe line and the activation of one sprinkler (4 in total) 


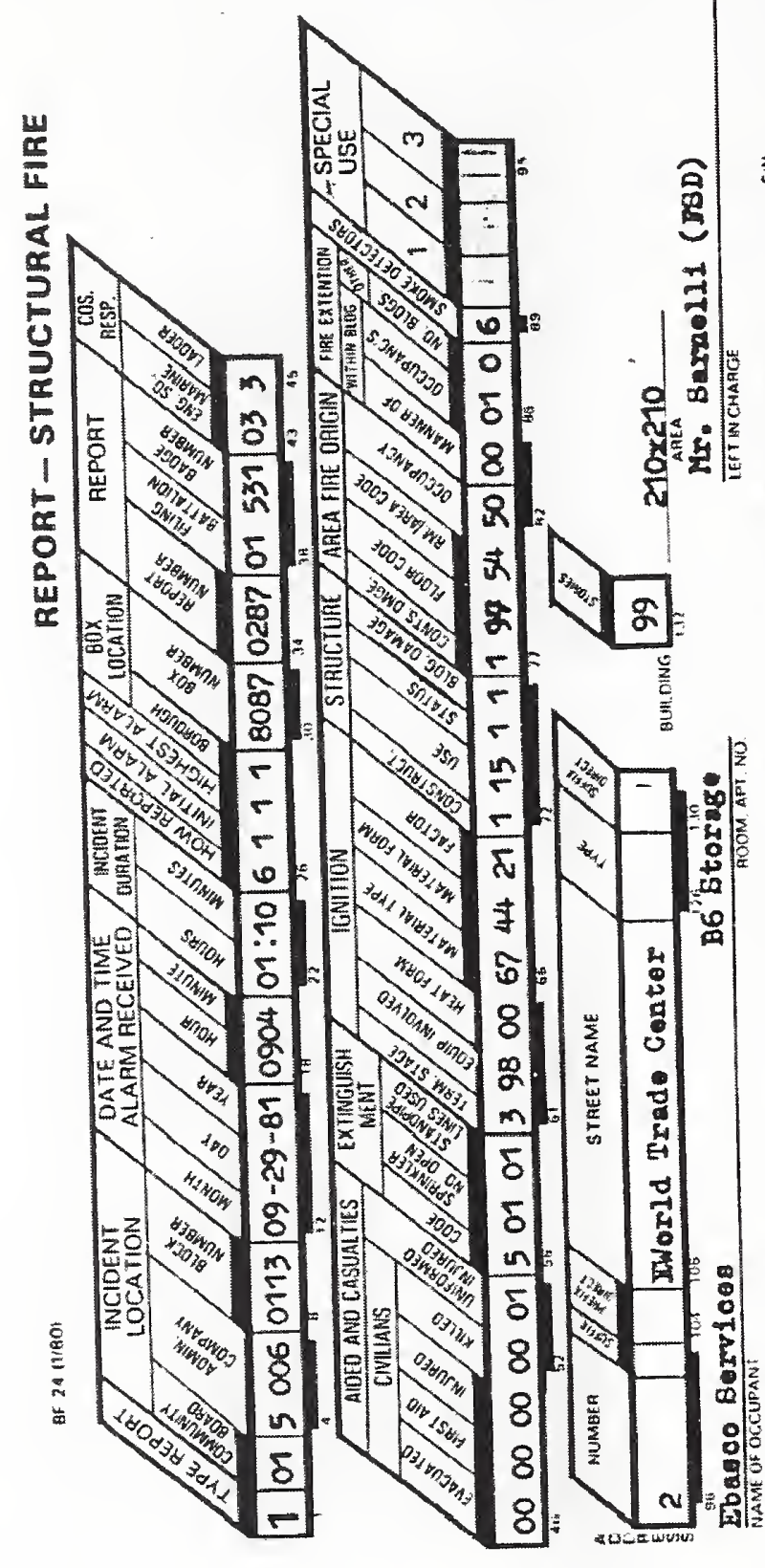

ACMINISTRATIVE COMPANY
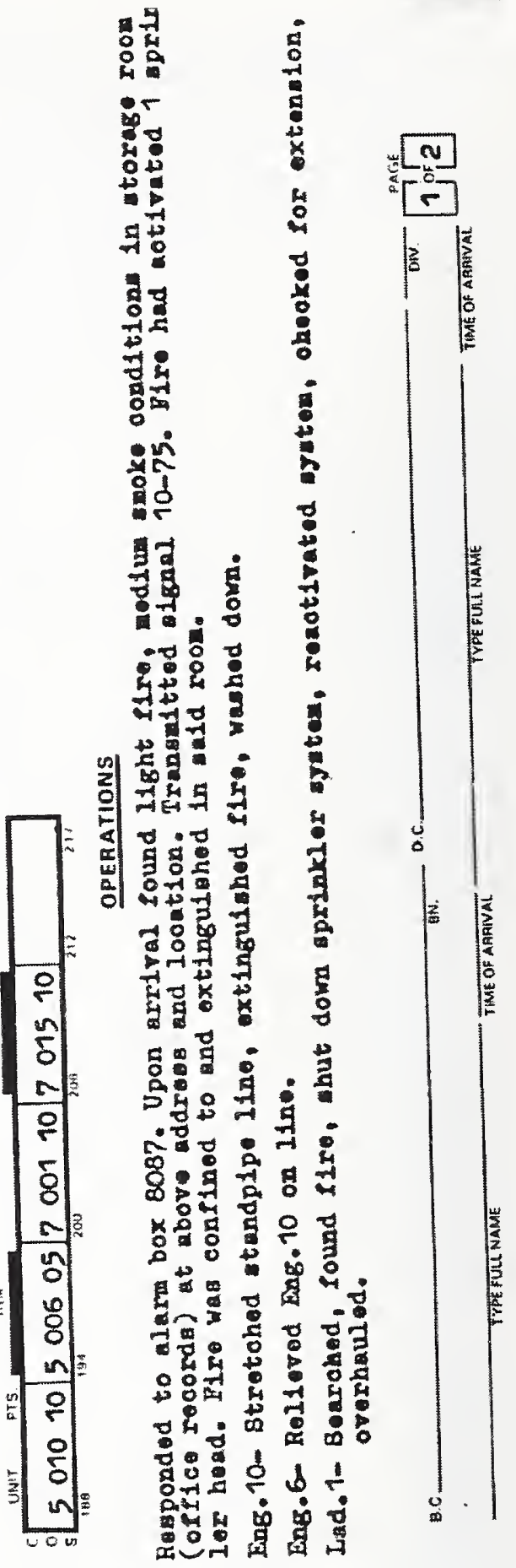

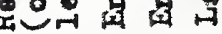




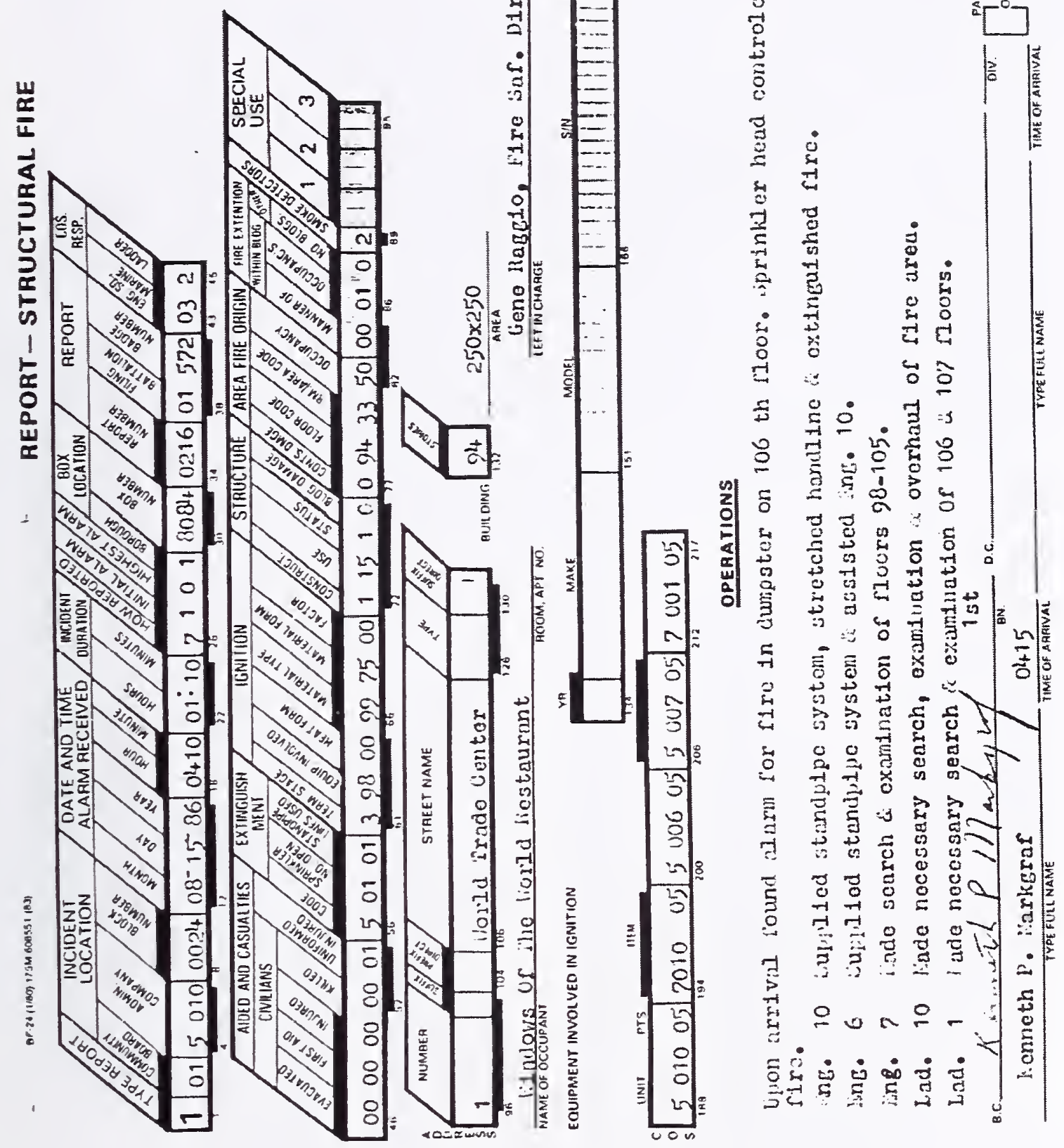




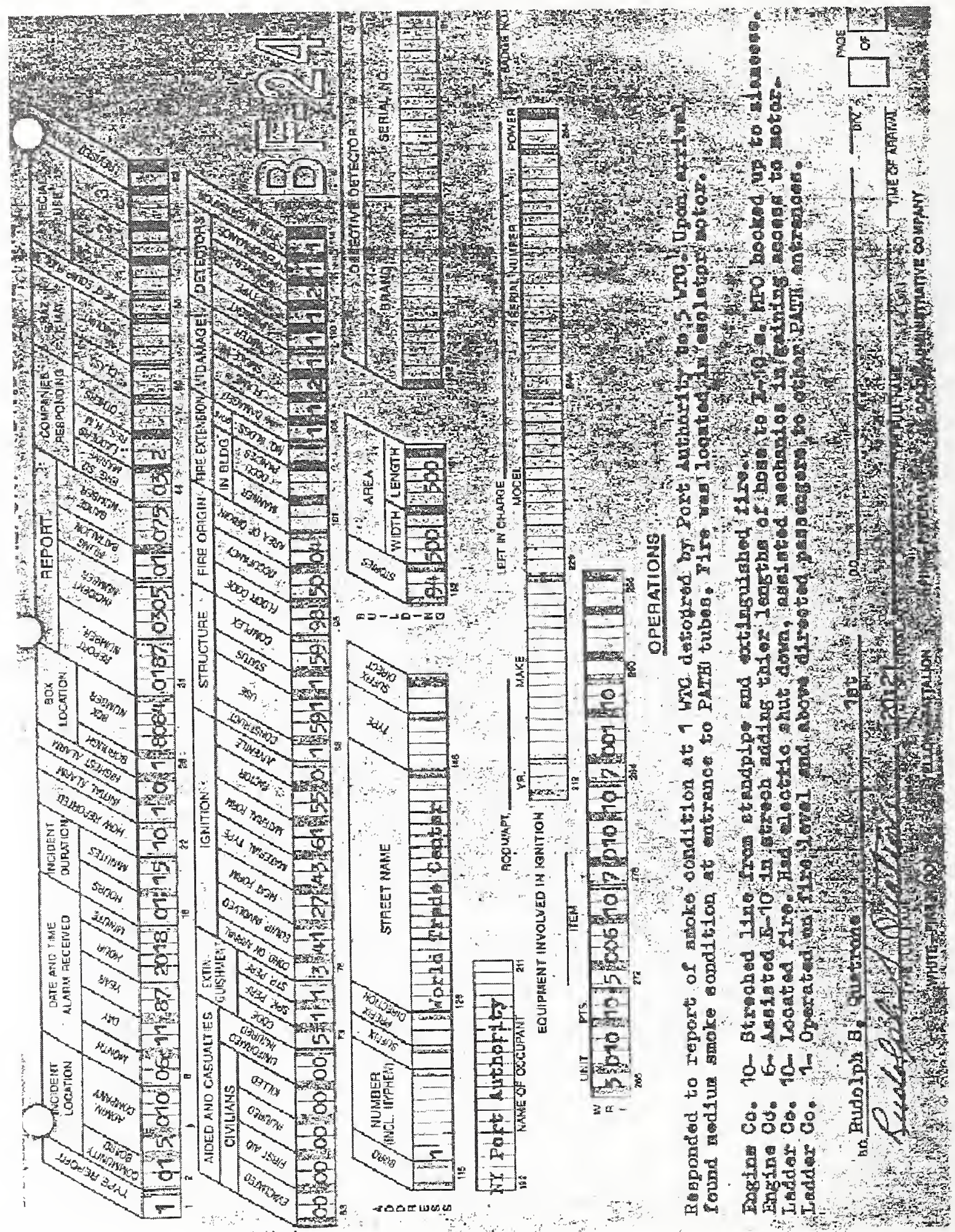




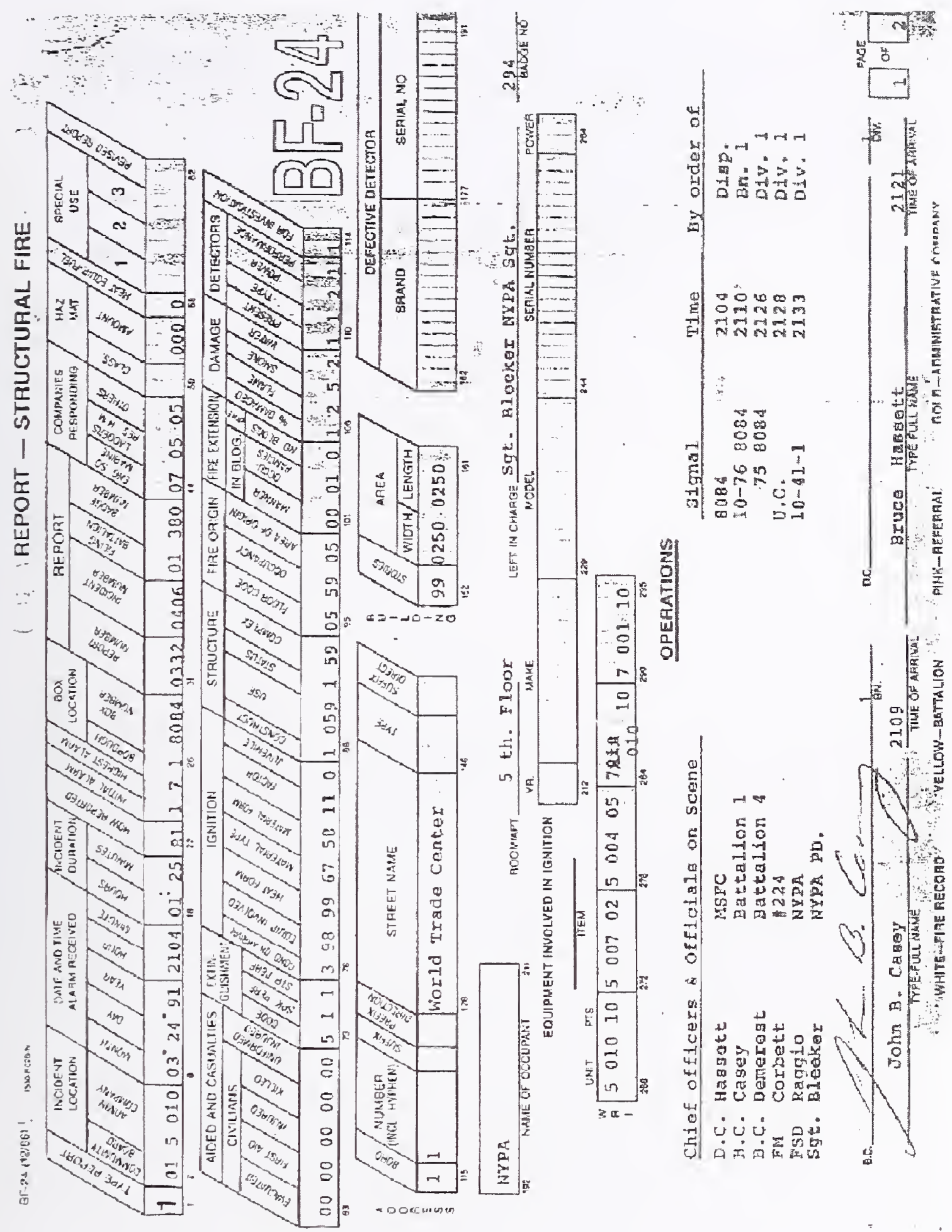


This page intentionally left blank. 


\section{Appendix G FIRES WITH STANDPIPES IN WTC 1 AND WTC 2}

Additional fire incidents involving the deployment of standpipe lines in WTC 1 and WTC 2:

- Fires involving the use of one standpipe line (27 in total) 

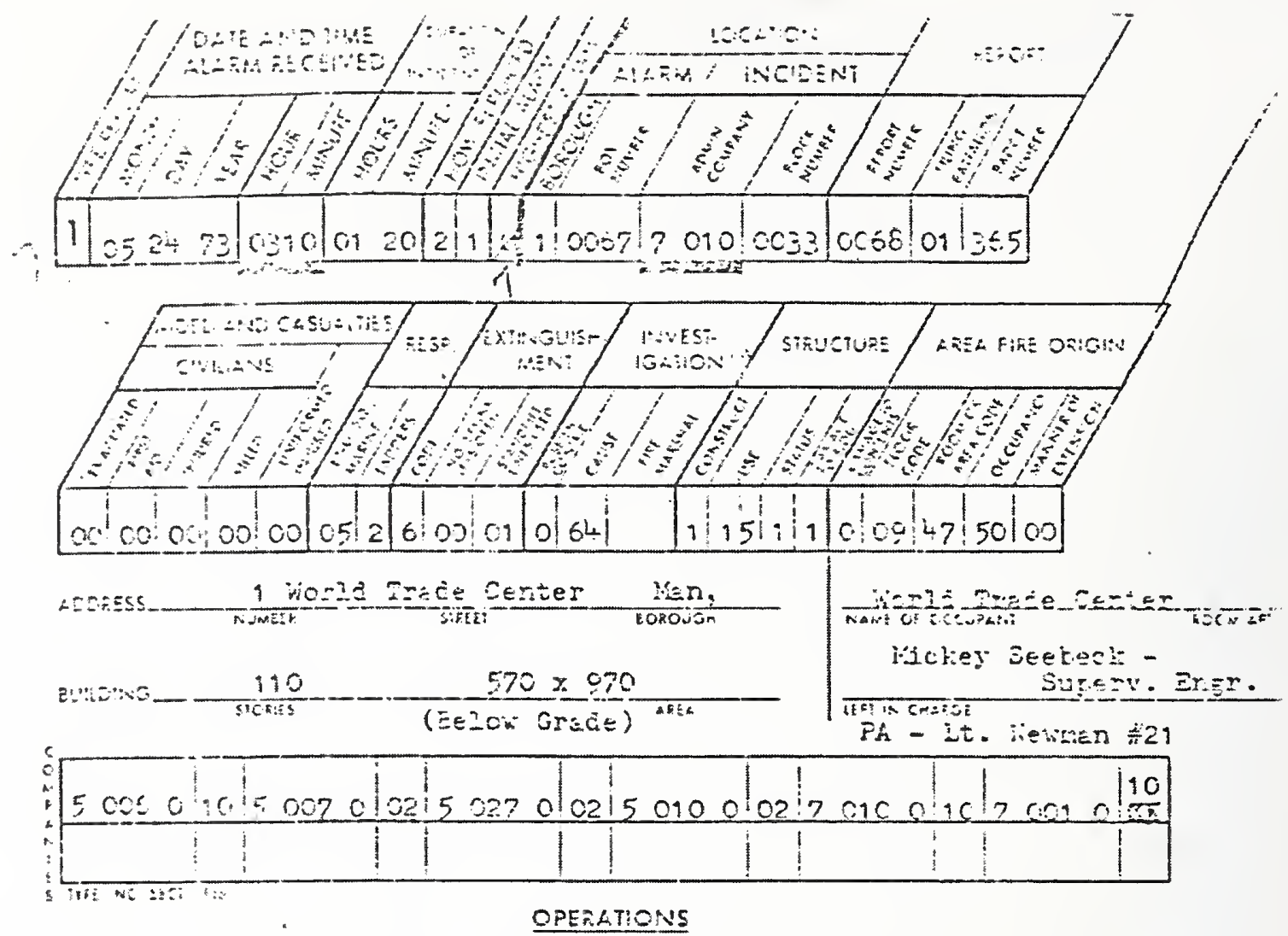

in crlivel found fire in elevator car (Jj) - E2 ievel, -J十 area -

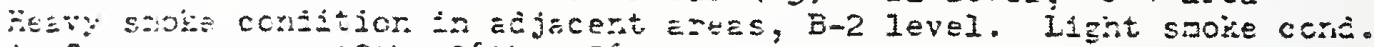

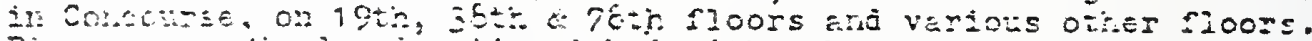

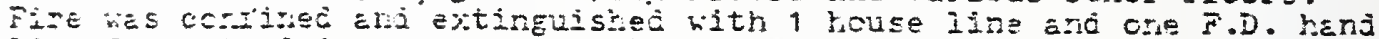

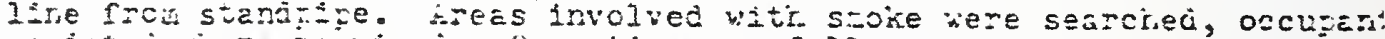
assistec hiere rejuirect. Oenaticrs as foliov:s:

Et- streach ine fros stancpire J-i area, ext. Fire in elev. d sheft.

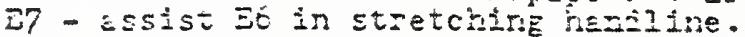

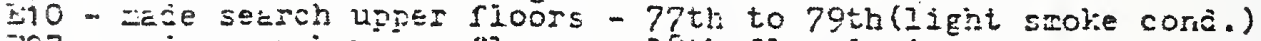

¿27 - sede secrcis upjer floors - 30th rl. Evic.

L10(its) - report to CP - search d examinetion of corscurse area.

ii - foree Elev. docr E-2 level, examine $\theta E T$, overieul.

B2 - surerrise operations of 26 \& li opering eler. car door \& ext. firt

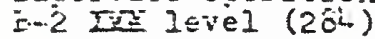

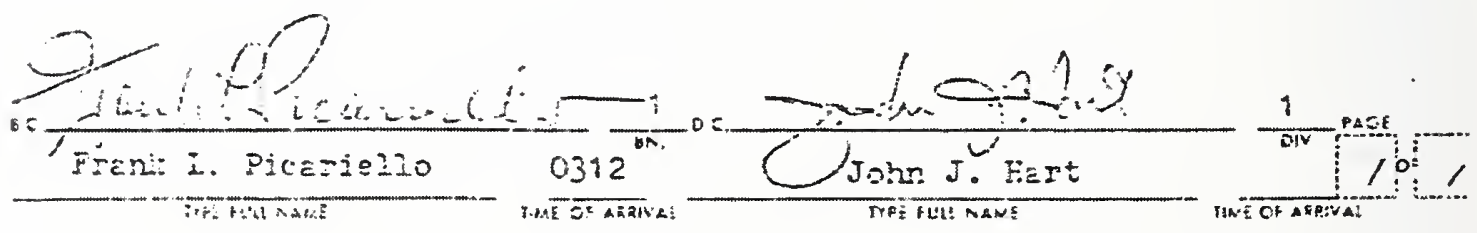




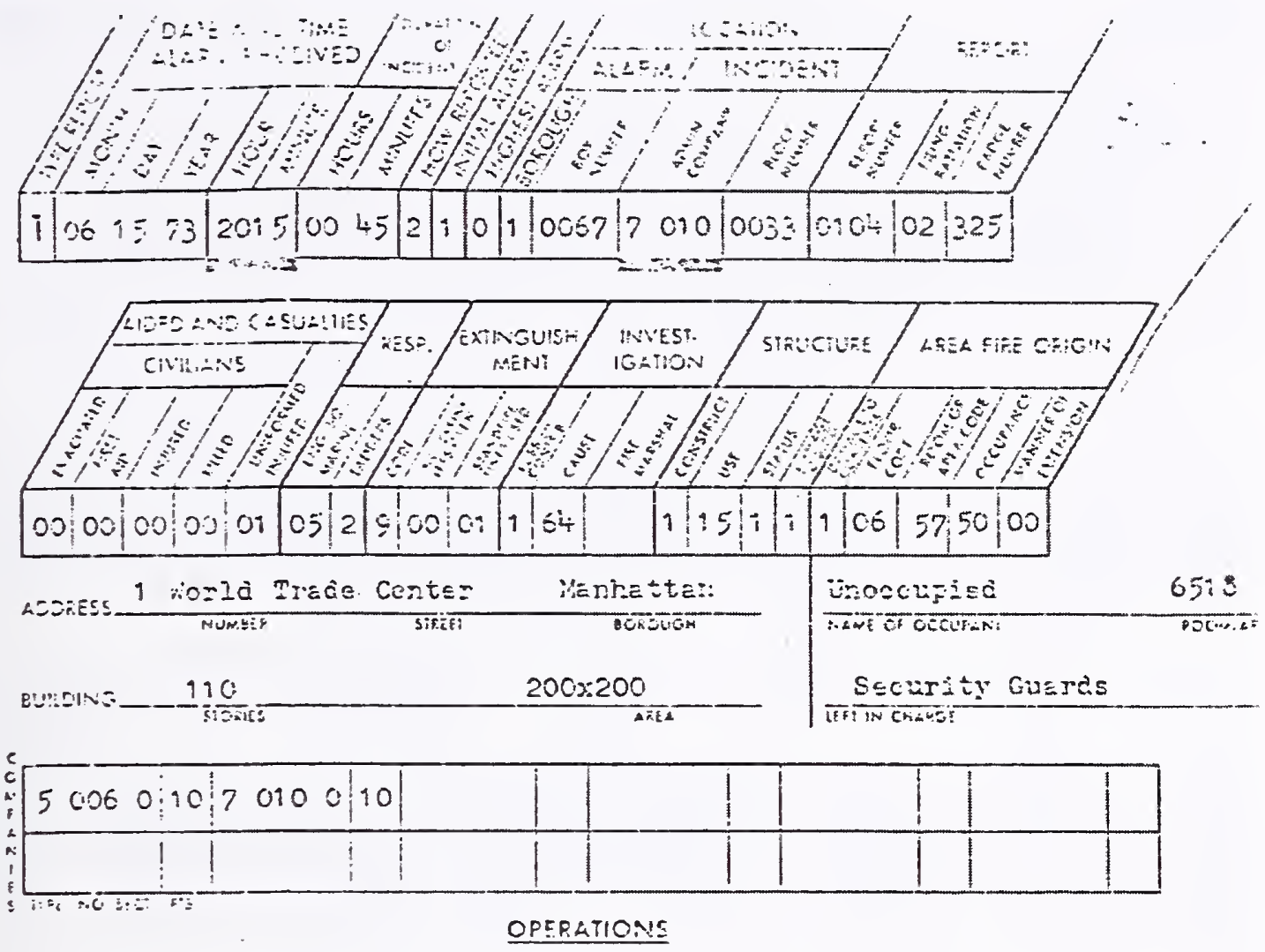

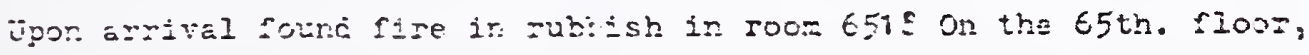

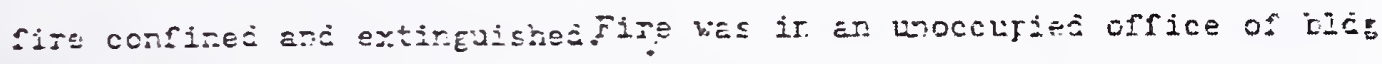
E.E: StIetched Iine off stang gipe extineuished. fize, had tarea rolled ups to 65 tin. floor.

i.10: Cv

M Scere: Fire Fttrol 范.

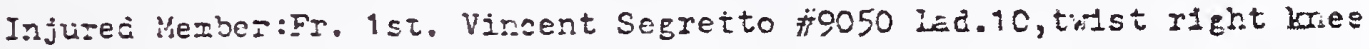
DI. Schwerts notified.rio tire iost.

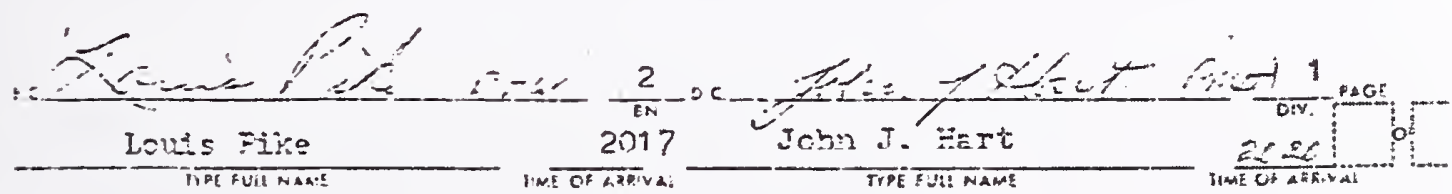




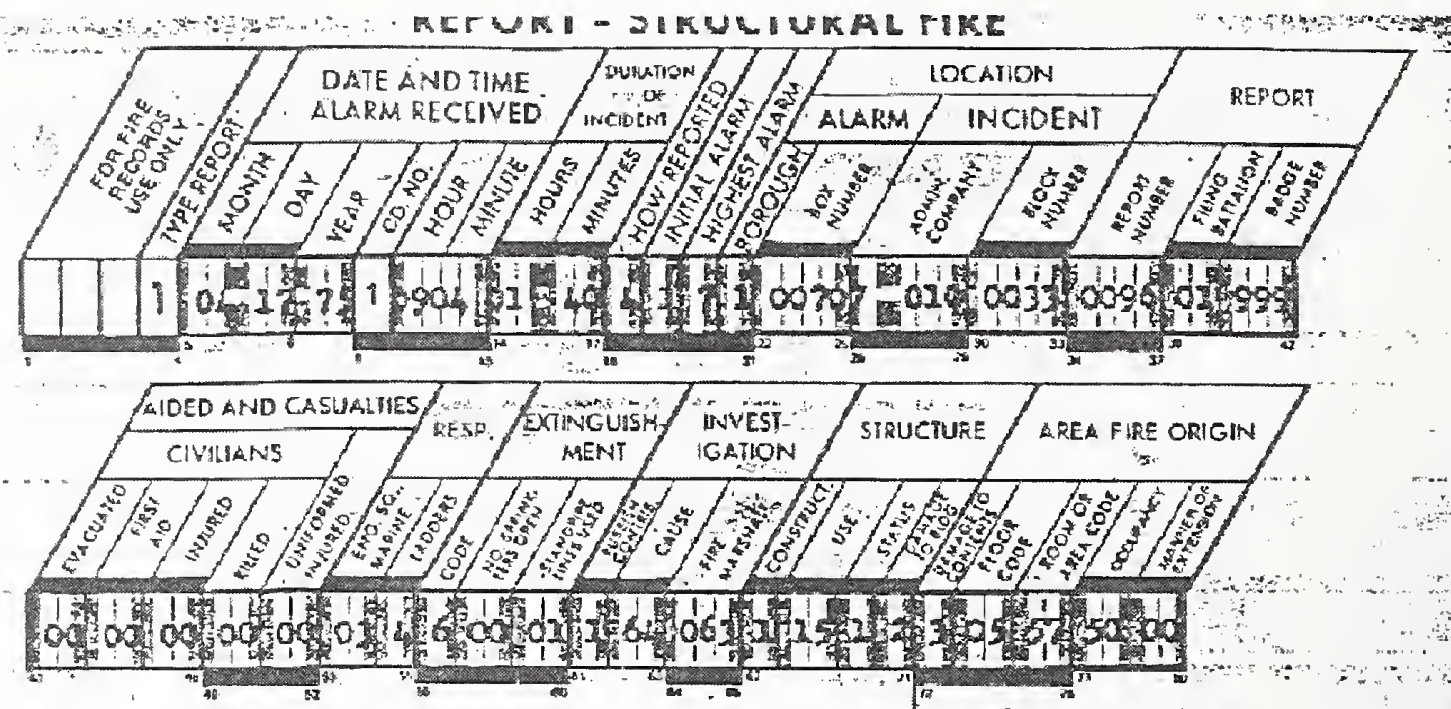

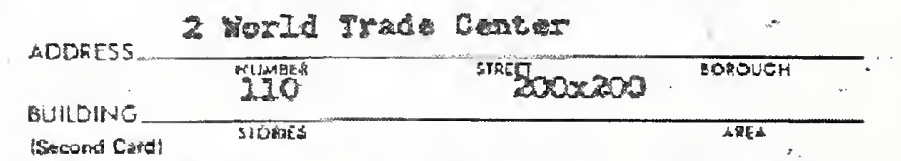

Port hutbority.

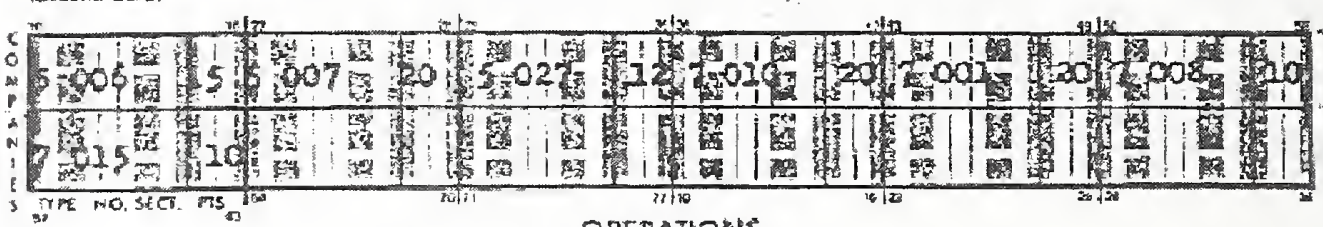

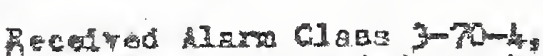
OPERATIOHS

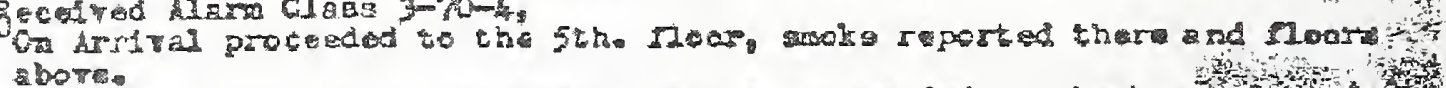

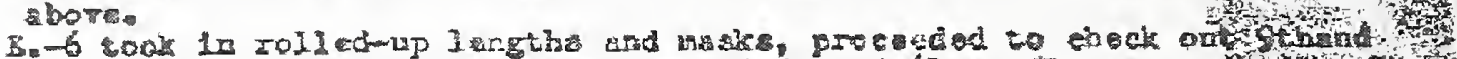

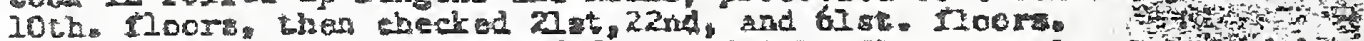

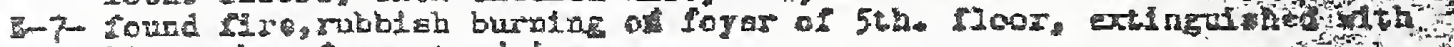
ine traker from atamipipe.

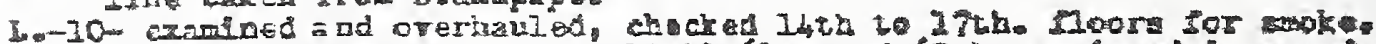

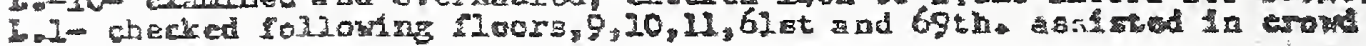
contral.

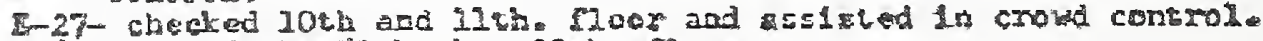

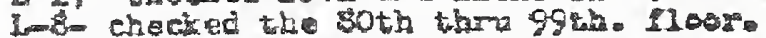

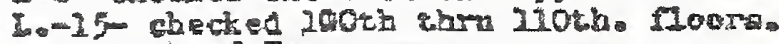

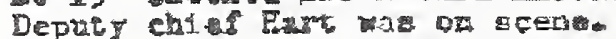

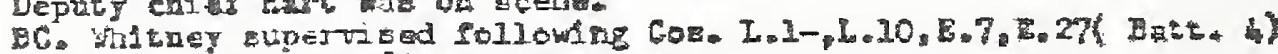

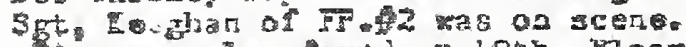

整

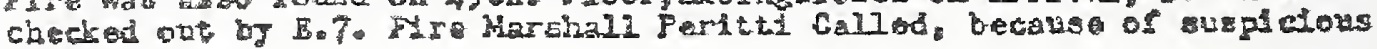
ISTs.

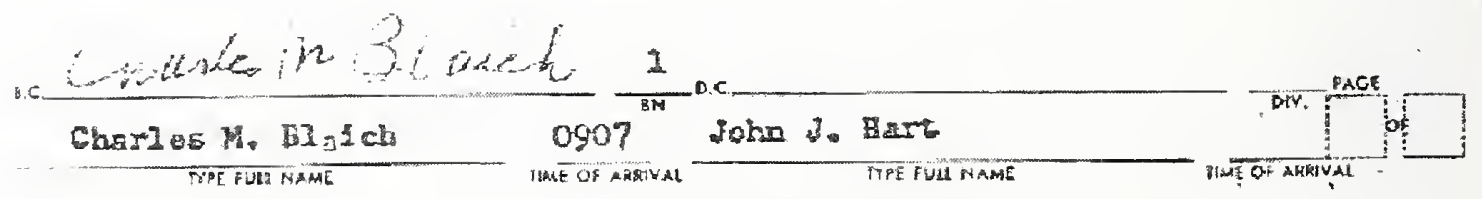


IARA

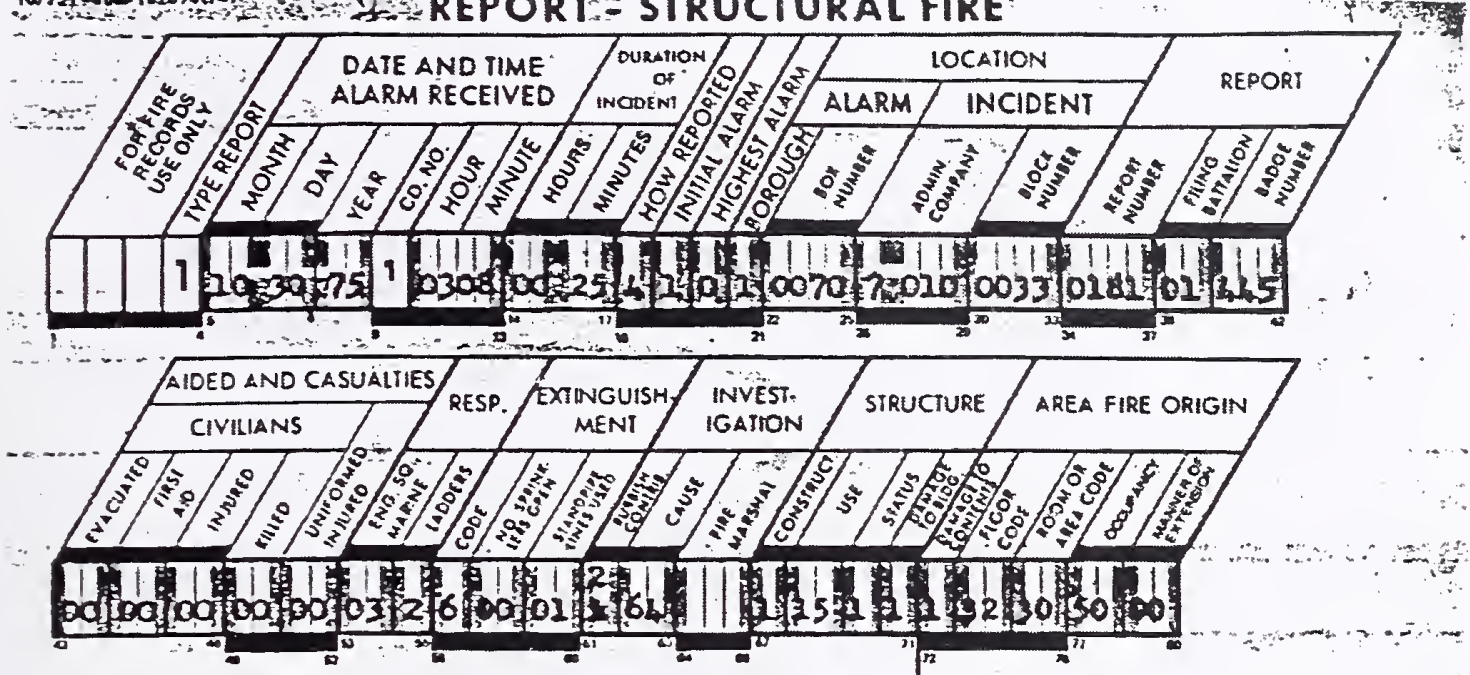

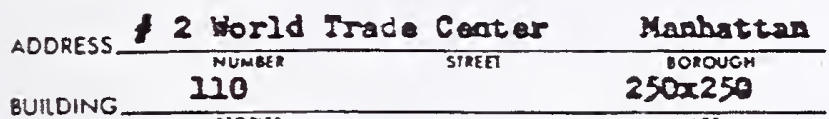

(seconat $C$ and

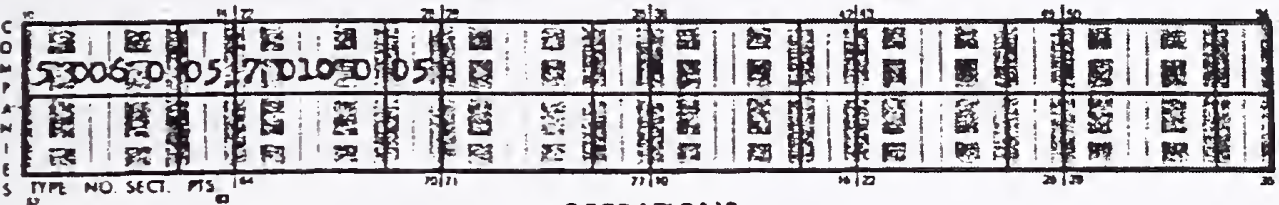

\section{OPERATIONS}

Besponded to Class 3-70-4

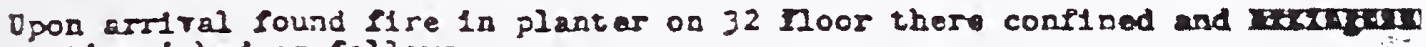
extingulat as follows.

E.6 - Carry 10 rolled up la: othe, hook up to S/P ontlet, etrecte I1ne and operate on and extingulat flre and necessary wash down

L.10 - Mato necessary exannation of 32 and 33 noors and ovarbaul burat debria on 32 Noor.

Port of A.I. Lathor1t5

NAME OF OCCUPANI

Mre Sefoty DIrector

IITIN CHARG 

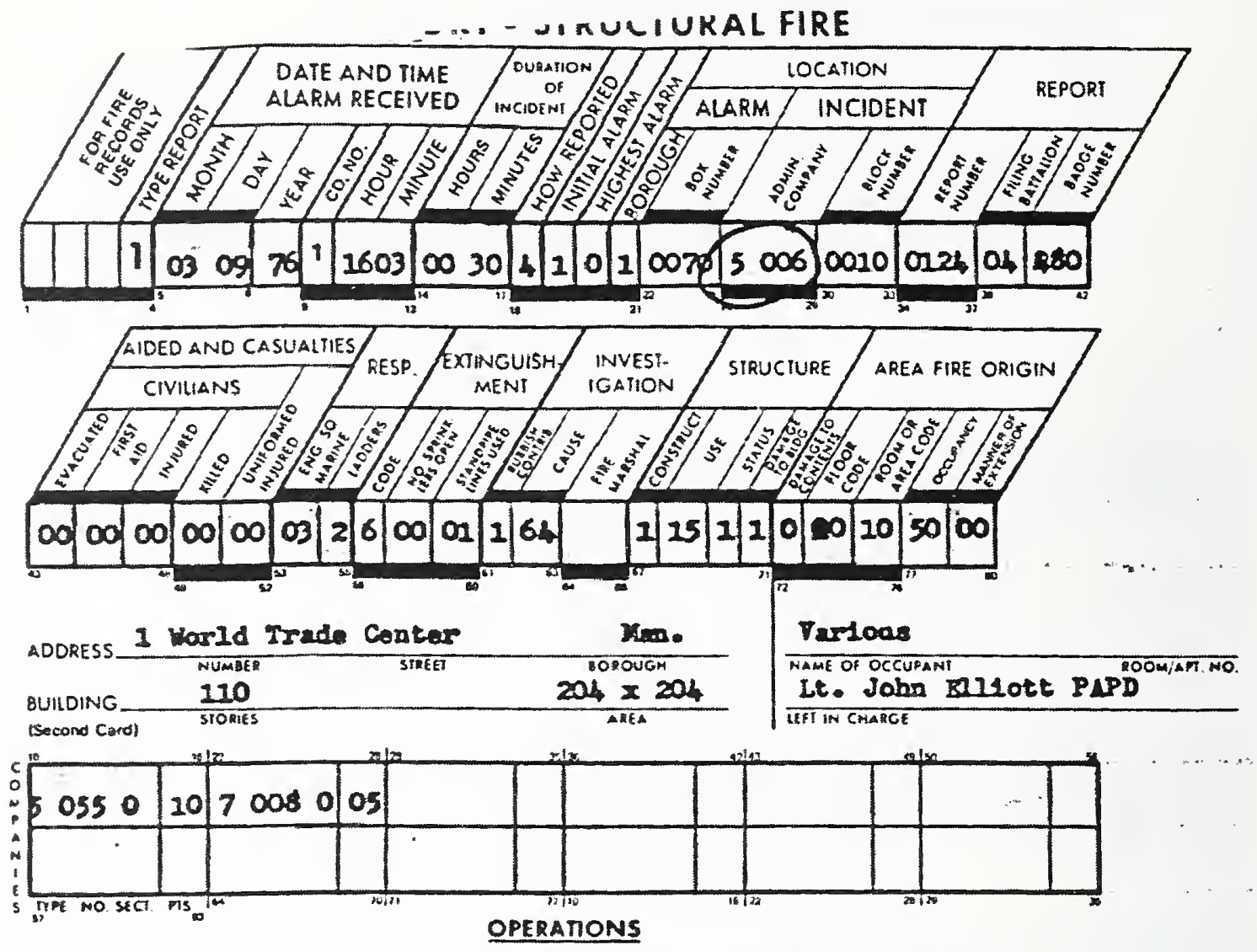

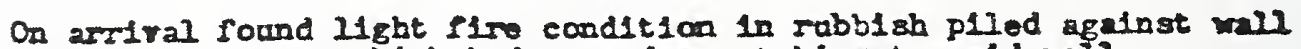
in mais concourso wich had caused scortching to sald will.

E 55 Stretched Ine from standpipe oatlet and extingalshed fire. washed down.

I 8 Exalnad for extension and corbanadod.

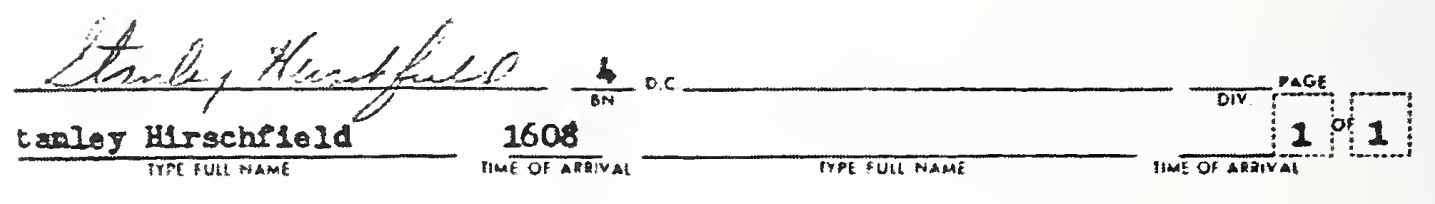




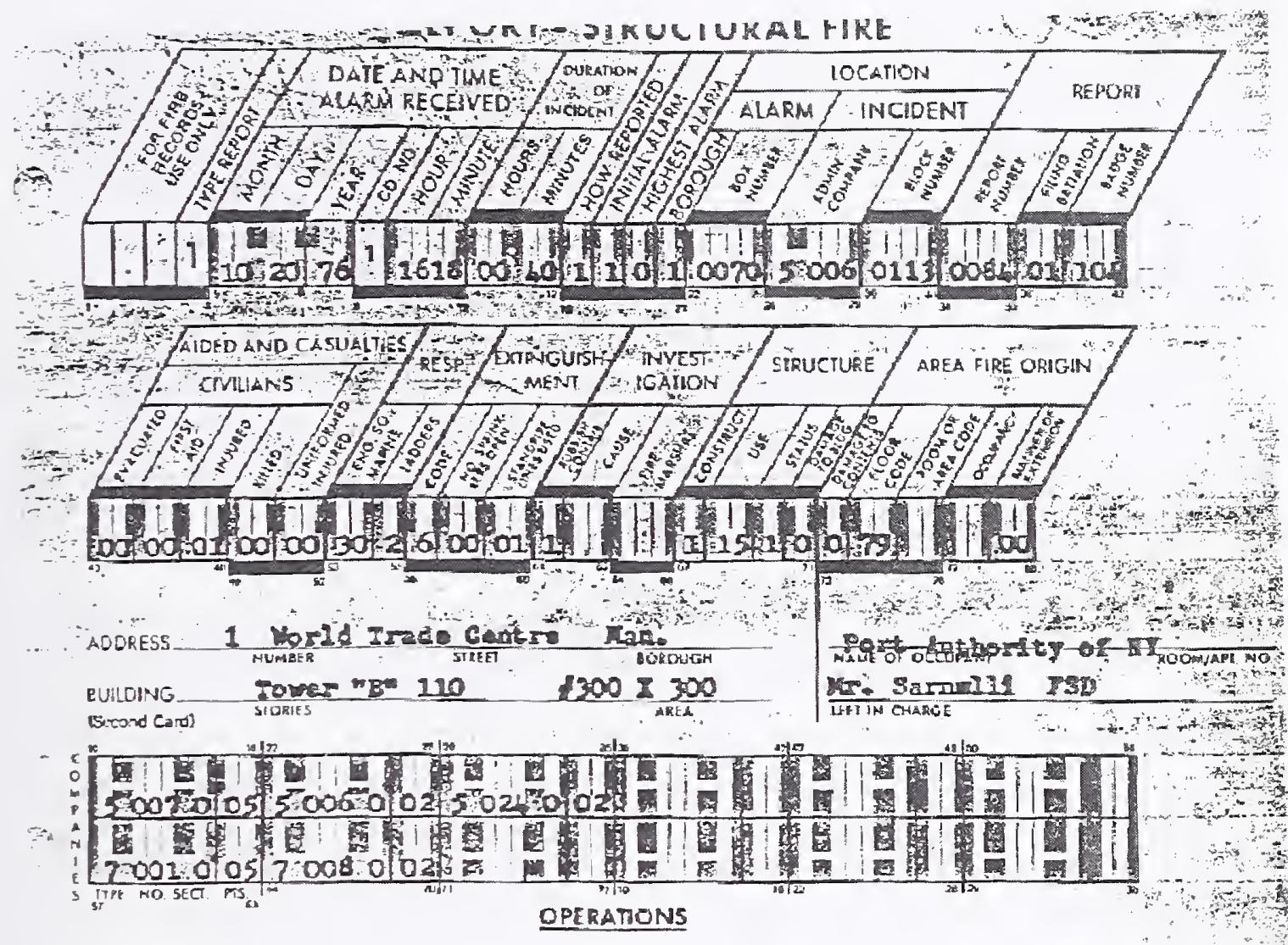

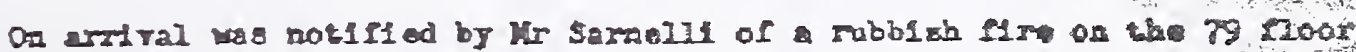
woutb wast cqudrent tover B.

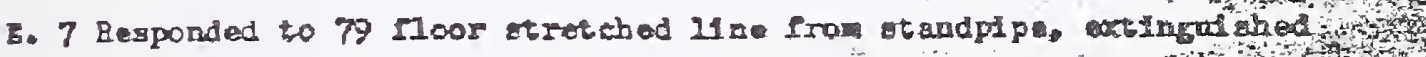
Ire

8. 6 Stretthed In and stood fat In IIre control conter arta.

Hot

I. 24 3tretched 11 no and gtood fast in atal moat In lobby' erea.

L. I Perforwed necessary search, rentilation, and over ball on the floort

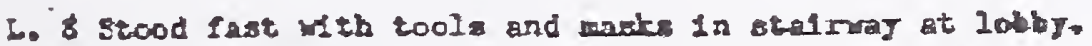

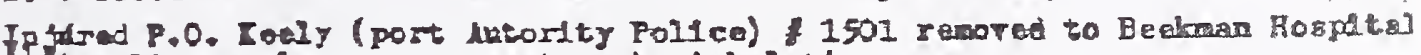

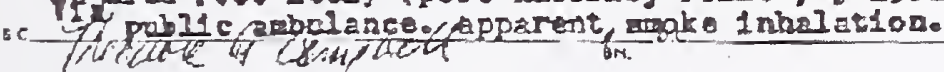

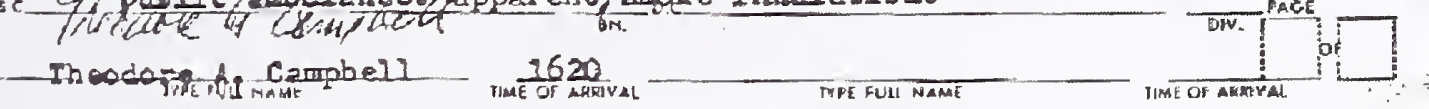

ADMINISTRATME TOMOANW 

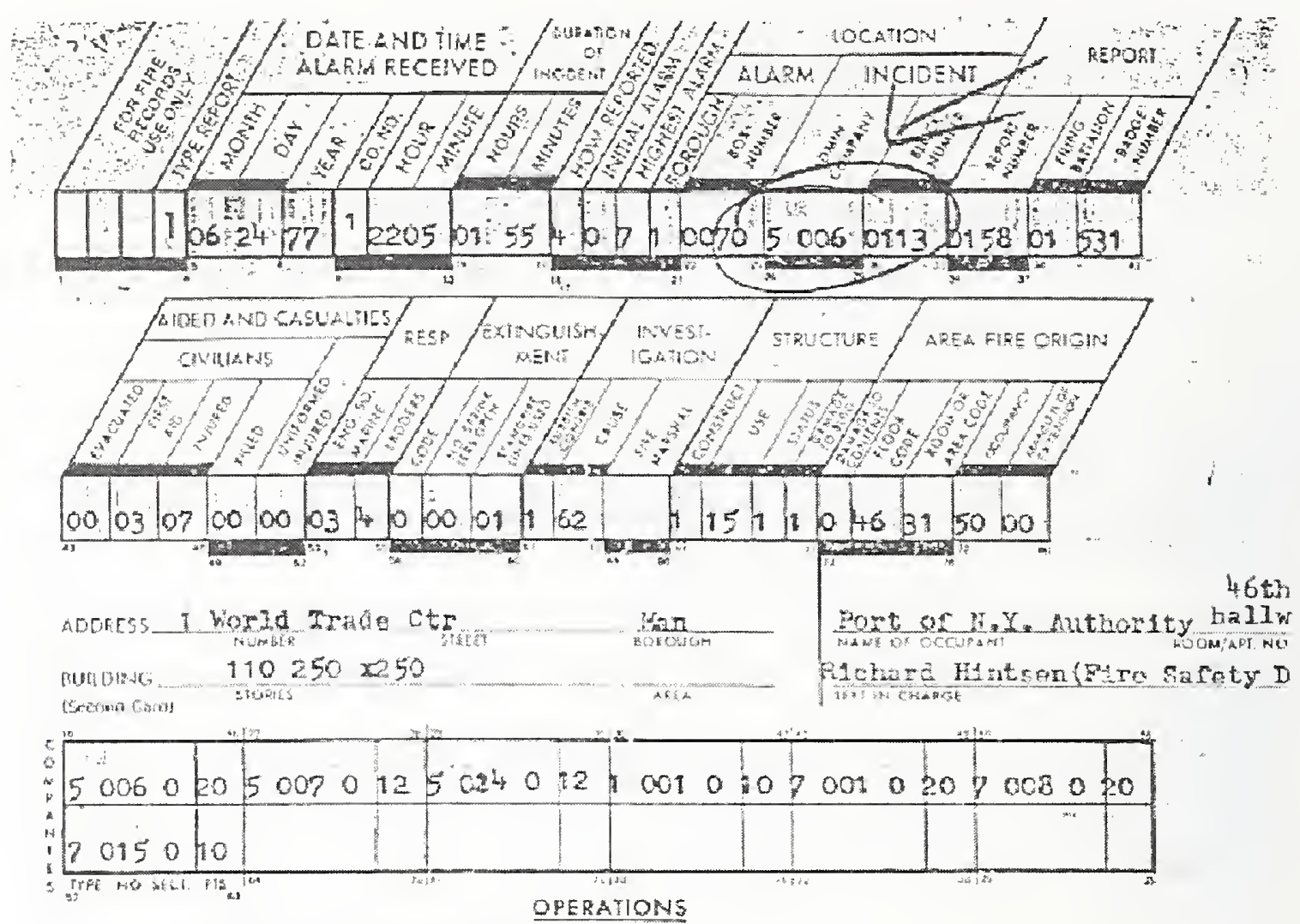

Responded to $j-70-2$ (ranual fiarto)

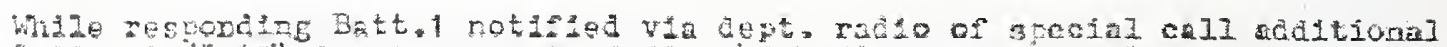

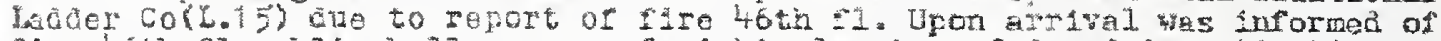

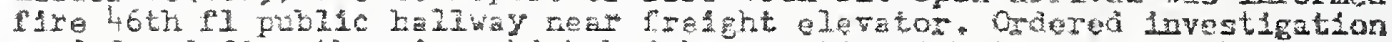

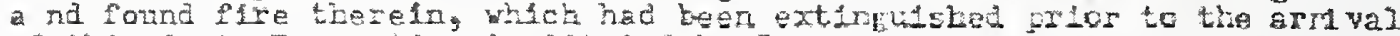

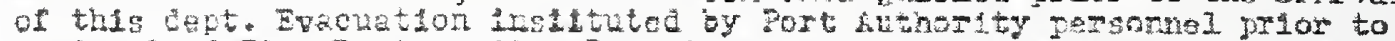
arzy 1 of Fire Dept. indts. Report of spoke detector operational of the 103rd 91. Fare located between srefght elevators 49 \& 17.

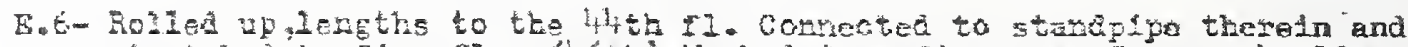

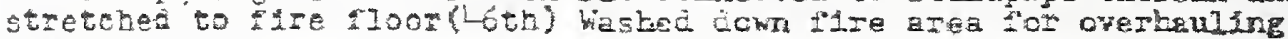
Firposes.

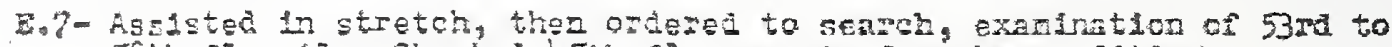

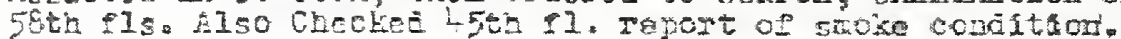

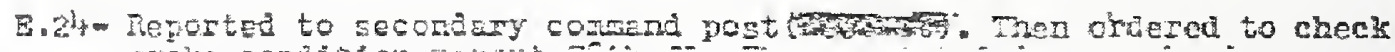
-

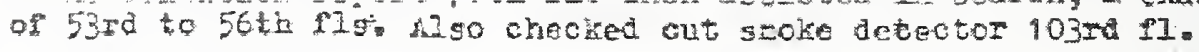

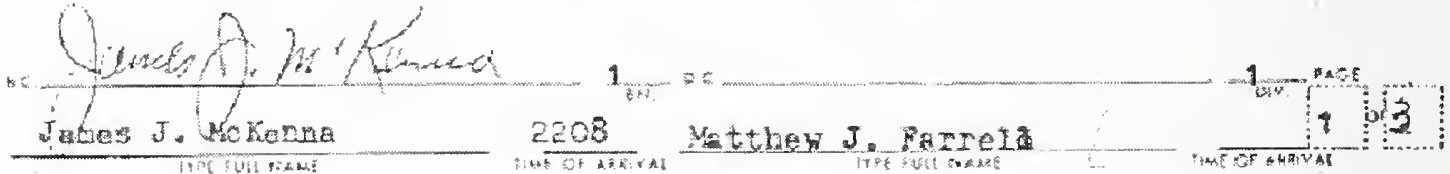




\section{REPORT - Additional Data}

- Structural Fire, Transportation Fire, Hon-Structural Fire or Emergency
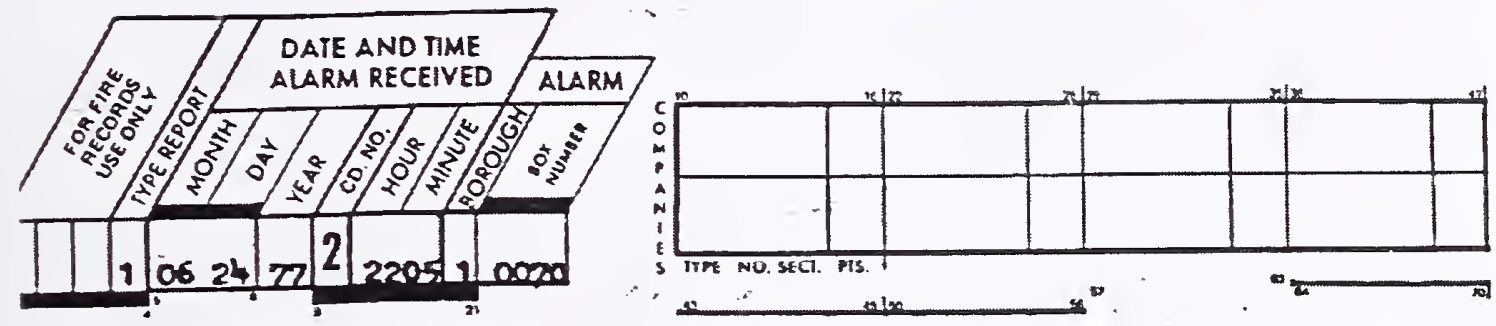

\section{OPERATIONS}

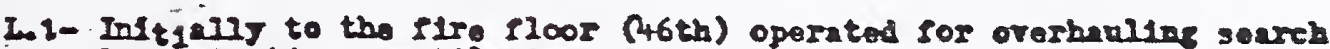
\& exaninationg rentiletion of sam. Then sekreh, cxalnation a

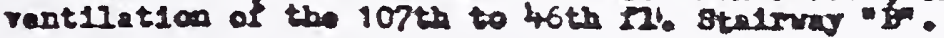

I.8- Indtlalyy to rocondary coanand post. (West st). Thon ordered to chock 78 th, to 60 th $18 \mathrm{~s}$ search \& exemination. 130 assistod in overheulins P15e. (1005 (toth)

1.15- Ipecial callod to report to West St. Thon trough lobby to socondary

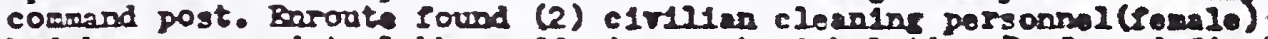
bed bon renored to-lobby sufferine sooke inhilation. Performed rirst 11d an 1ajared elv1lians. Then relleved by Bas. I with resustetatar

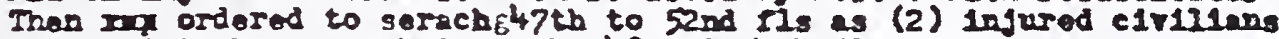
reported to he re varked on tho $48 \mathrm{th}$ i $50 \mathrm{th}$ 51s.

Bes.1- Belloved I.15 and admistered risst ald (Inhelation) to injured elvilans. Then ordered offlce i a reminder or menbers vent to

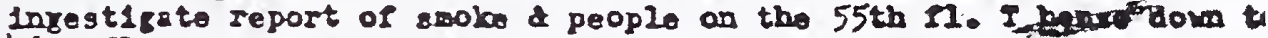
$46 \mathrm{th}$ II. Soxrch, exs anation of floors anroute.

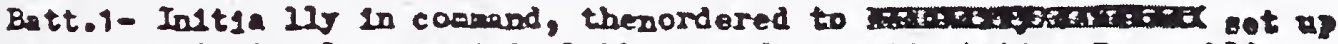
additlonal comand in lobby, as l1rst ald stetion. Tro public abriaxes stand ine by with (4) rosnscitators. Directod search operations of B.7, L.15, Bes. 1. Ordezed swoke parce 45th to 107 when tire vas out.

Batt.2- Ordered to superv1se undts on the 11 re floor \& report conditdons there1n. Supervisod in pert operation of B.6, L.1

Batt.32 - Ordered to sepervise operations of unlts above slre 11005. ickmes . M' feence 


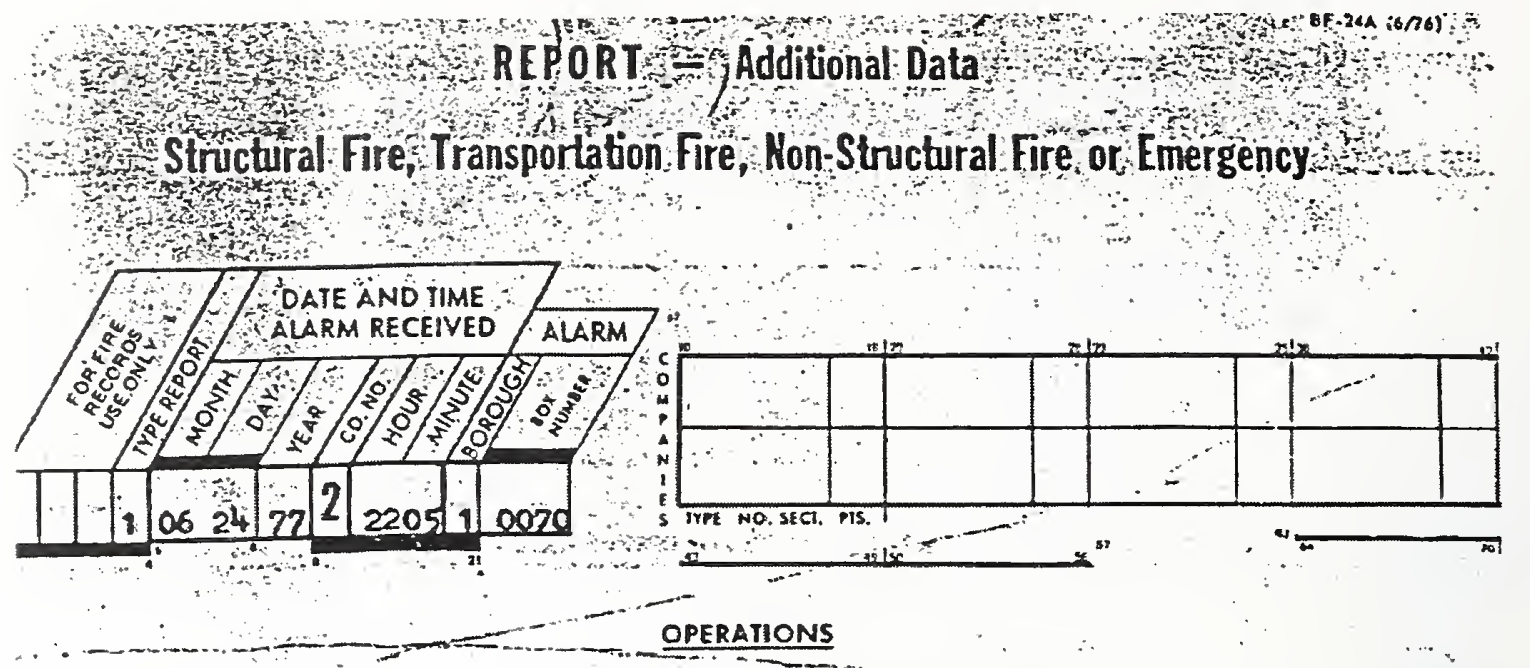

Act Asst Chiol hur on the scene to supervised overall operatinas.

Hemarisg Lo l checked all elovator shr ca Is before leaving s=eno: Ortered 10-4t Code 8 due to report rrom Port Anthority police of Iabo: trouble with Helotalnence personnel(Temco)

Dept. Photgra pher ordered to scene to tako plctures of fire area.

Alaza vas tarned in by 14. Nick Cappola, Temeo Matitajnence

(2) Beelan Ambulances on the sceno ander is. B. Jons

Injured C10111angs
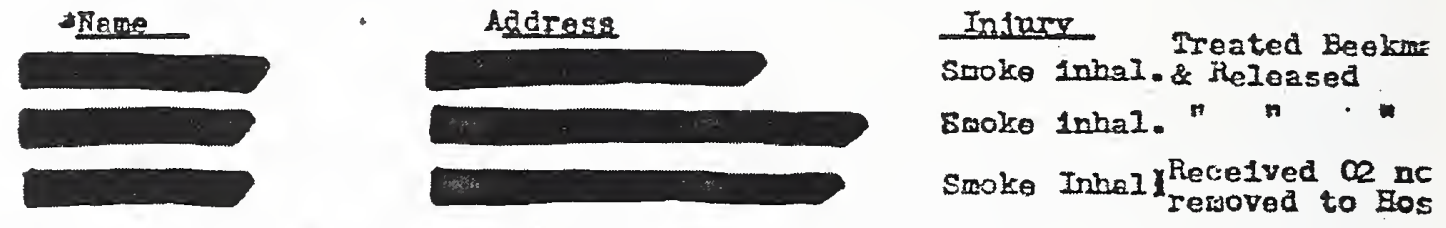

liote : All of the above are enployees of the Temeo Co.

Sgt. Steve Fax Sdif 264

Burn to Fingegrs Rt Iland.

PtI A. Halleter

Barn to Rt Wr1st

PtI Hejers

Stroke 10hal

PtI. Careale

Smoke Inhal.

Bote: Pollce nemers of Port Authority Pollce Dept. All infured treated at Beerman eosptar soleasod.

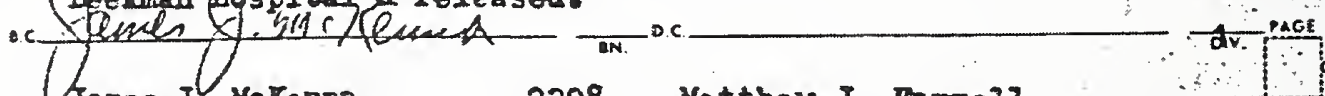

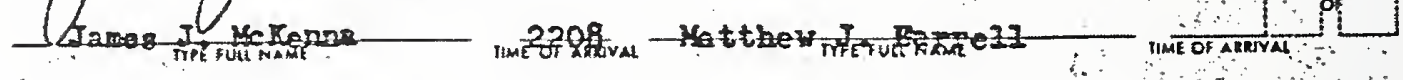




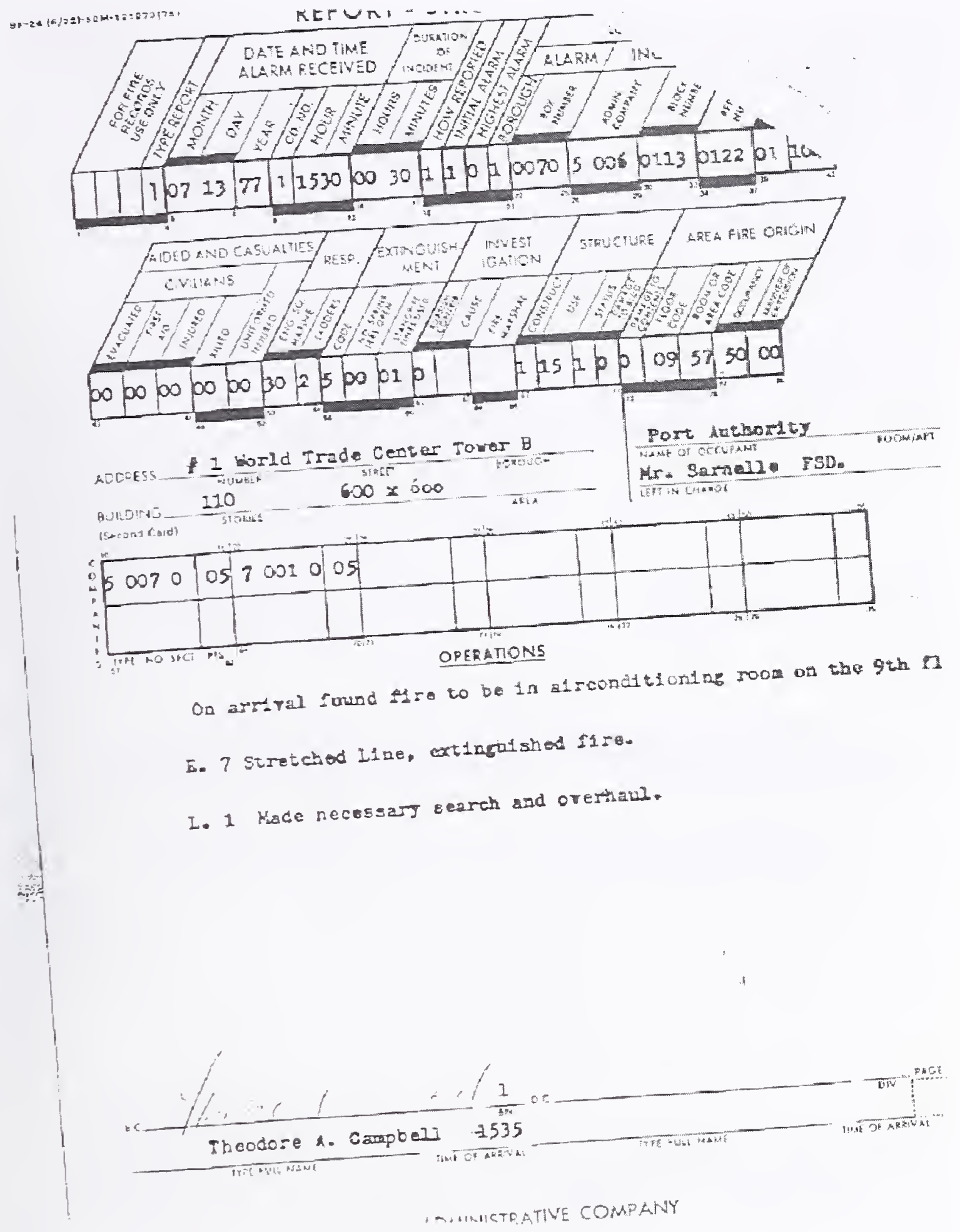




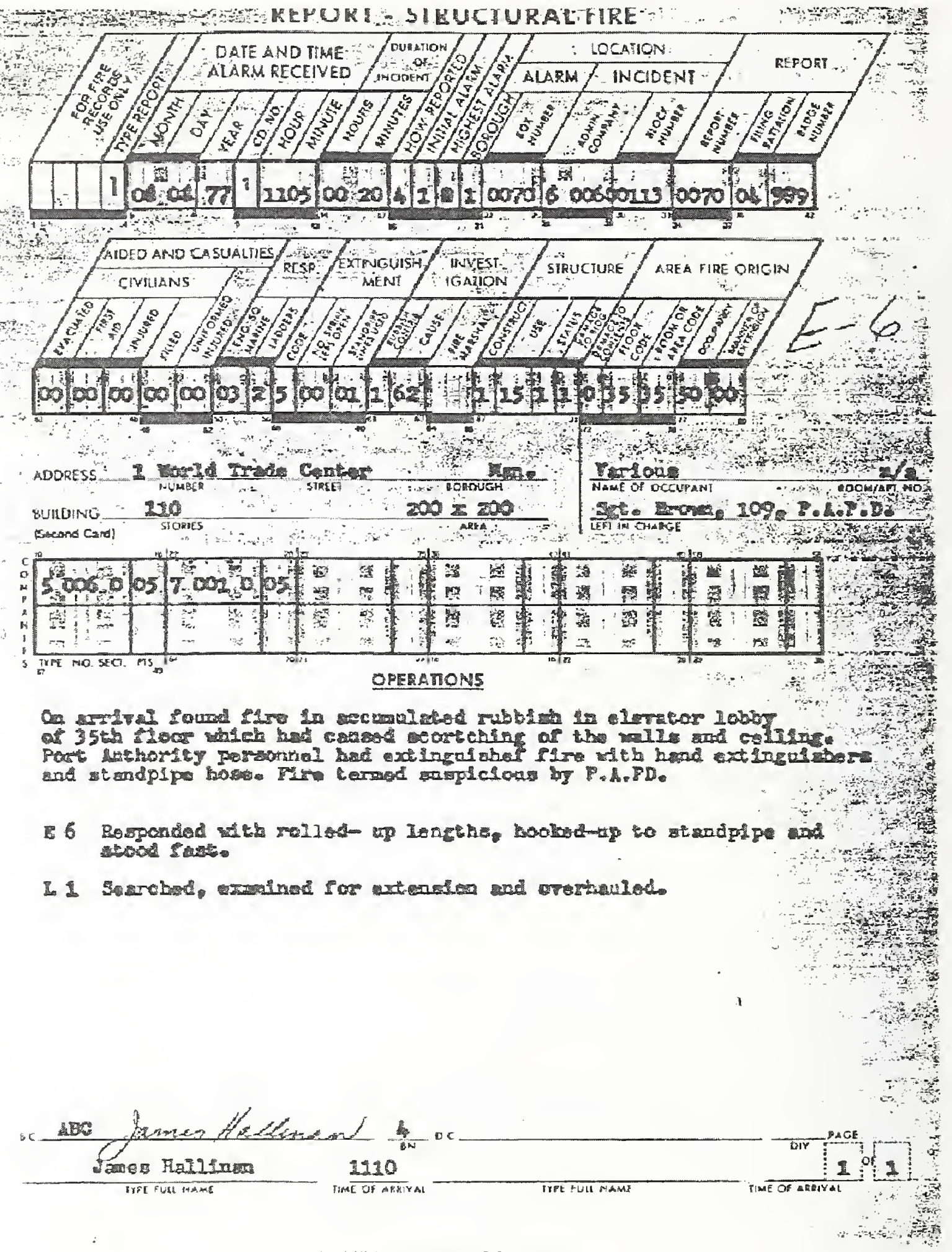




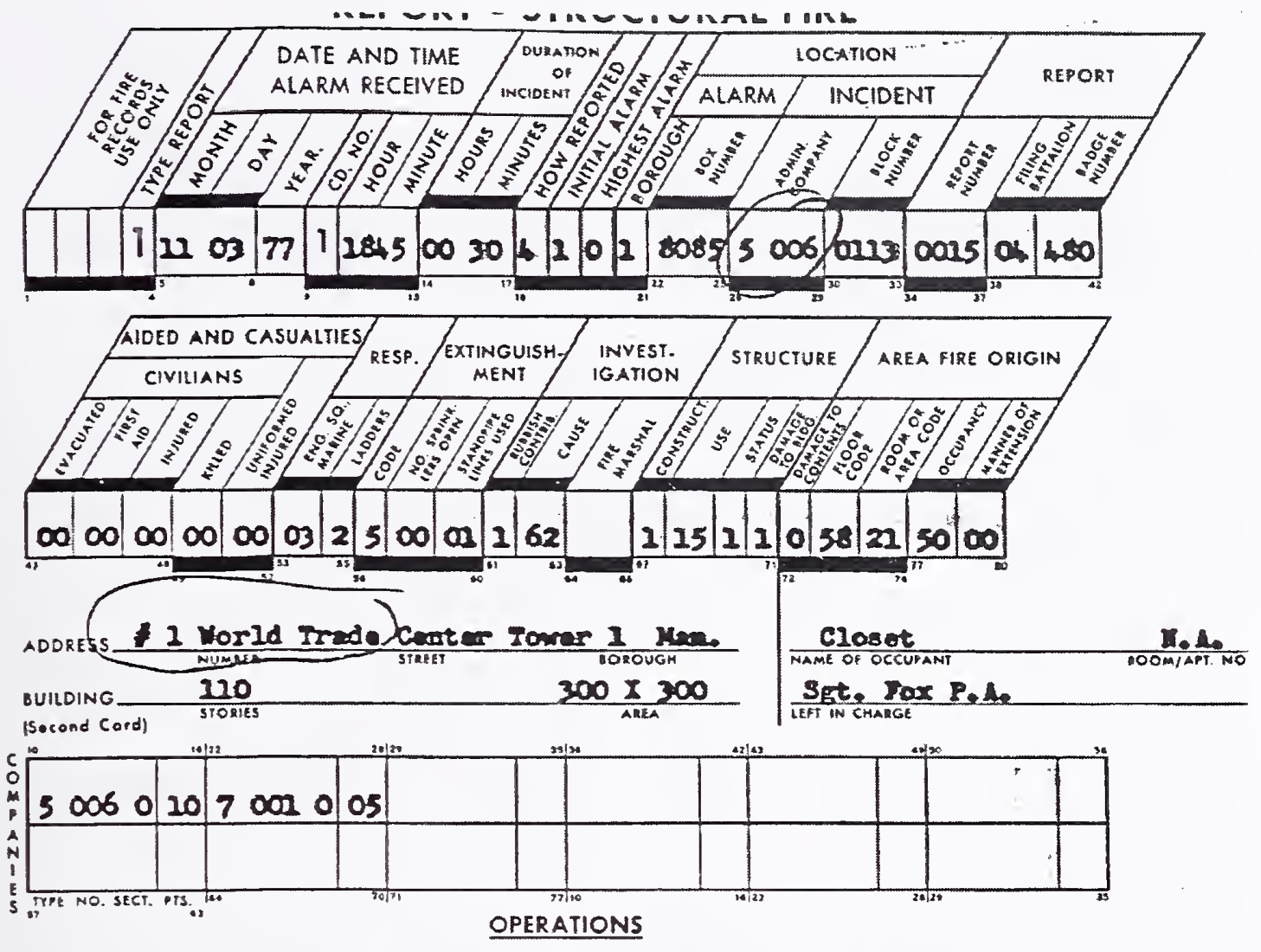

On arrital found canse of alare to be flr in jantorlal closet 17 rolring rags and rabblah. Batt 4 roquestod a 1041 Codel F1re cauged searlag of palnt on will and celilns.

I-6 Stretched rolled up lengtho and hooked up to atandp1pe outlet and axt 1 olshed Ire.

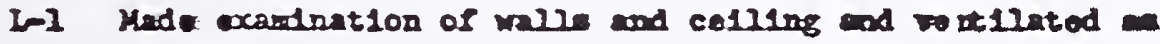
nocoseary.

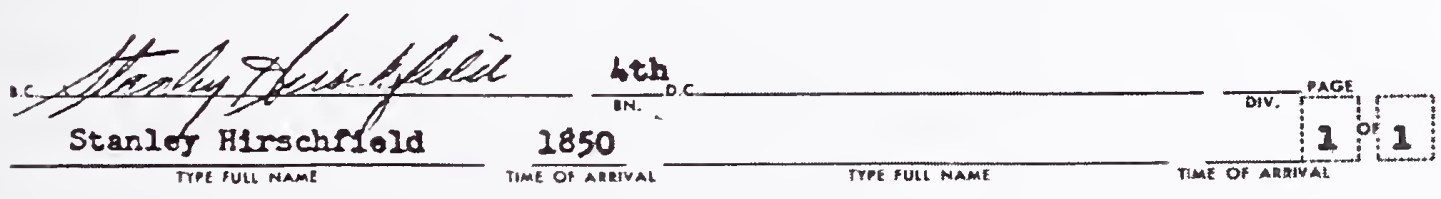



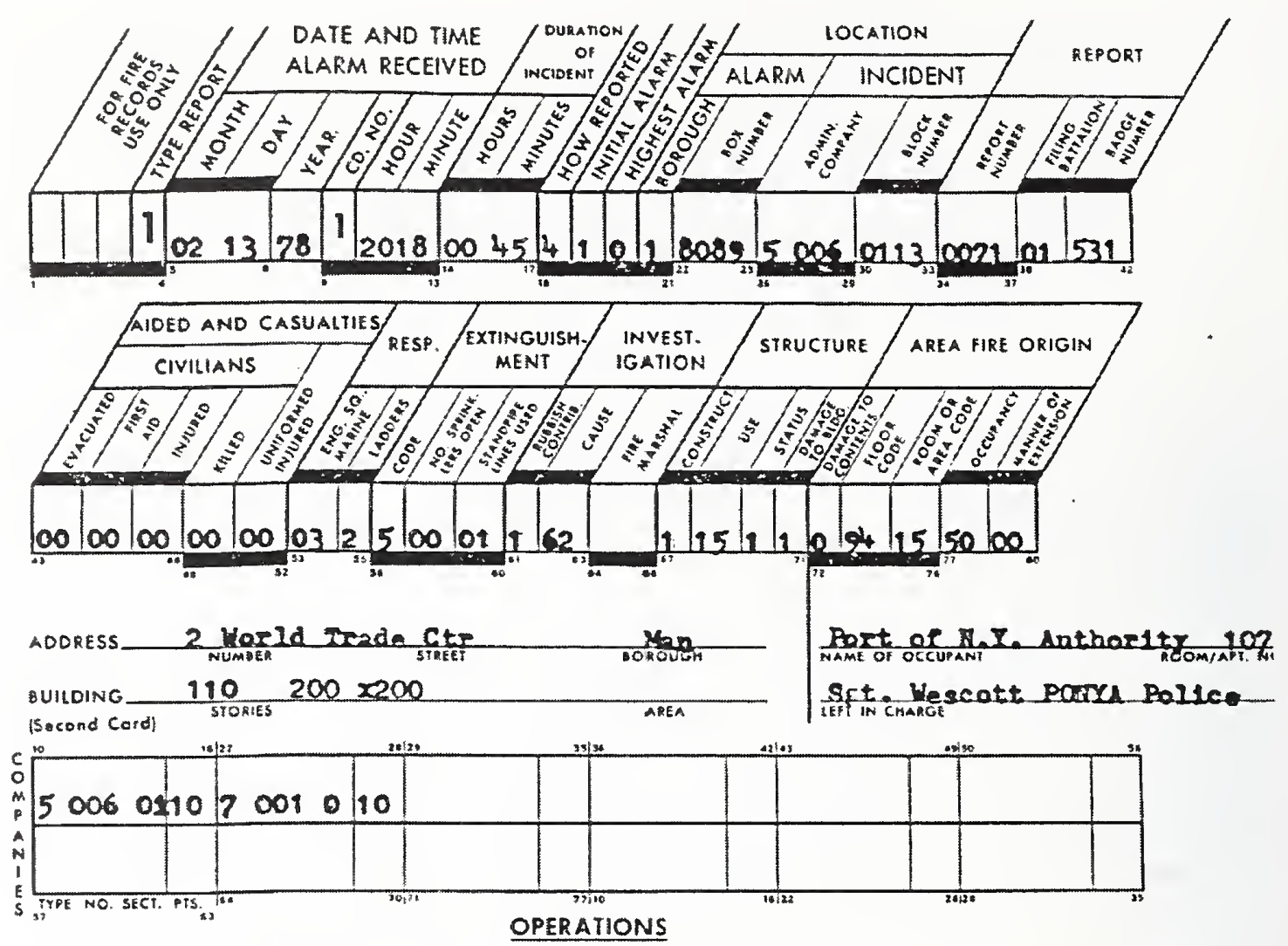

Hosponded to andal la ra box 8089

Upon arrival ras inrorand of flre $107 \mathrm{th}$ PI. Ordered investication and found PIre in rabish a aalnt. matarlals thereln. Batt. 1 ordered adoltional Battallon Chlef to respond on report of derinate fire. Bett. 4 responde d.

F.6- Bolied up lengths to tho fire rloor ext1ngulshed remaintor fire

L.1- Seasch, examination of IIre Floor a floor above. Opared ralls for exarination. Overhauled burned naterials.

Bo.4- Ordered to supervise operations on the flre floor.

D17. 1- Responded to scene, and assumed counand.

Note: Bett. 1 transilted 104 r Caje 2 \& requested F.M. to respond.

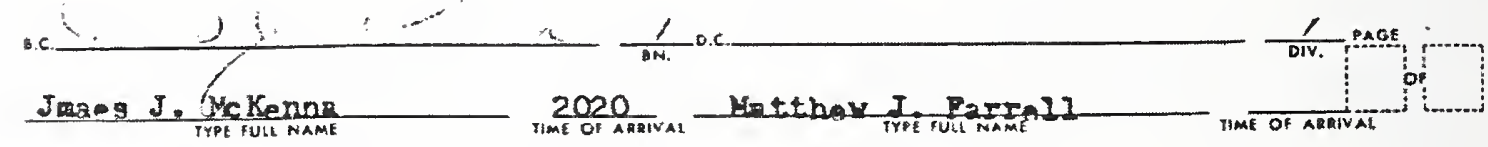



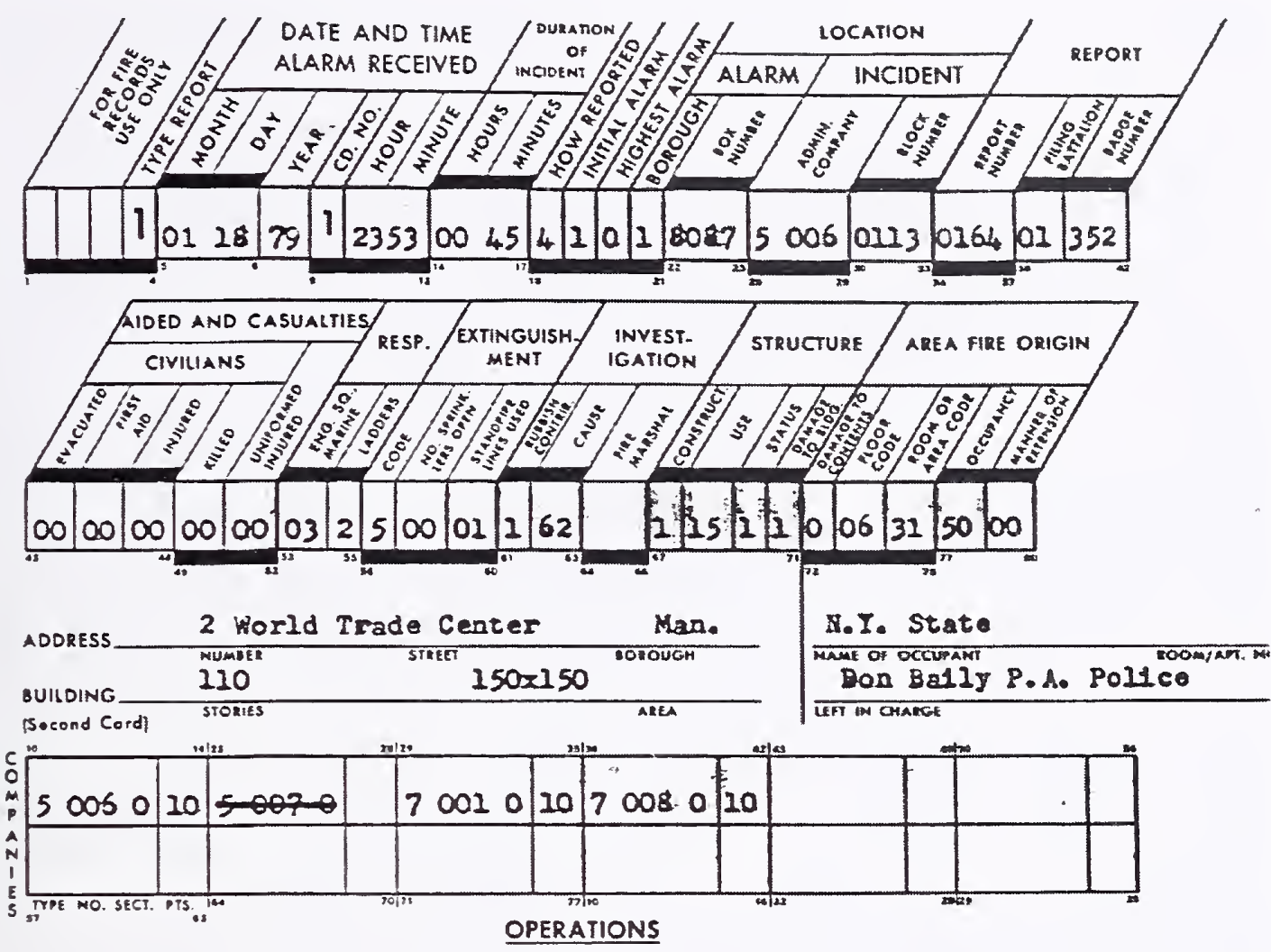

Upon arrival was told of flre on the 6th floor, operations as follows. Ladjer 1 made necessarg 1 mpestagation, located the slre, vented, overhauled and searched.

Ladier 8 searchod and vented noor above, overhauled.

Engline 6 stretched a line from standplpe and extsnguished the f1re.

Engine 6 washed down.

Batt. 2 on the scene.

D17. 1 on the scene.

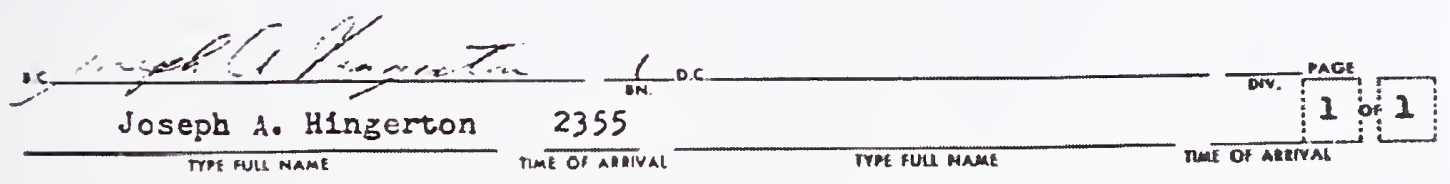




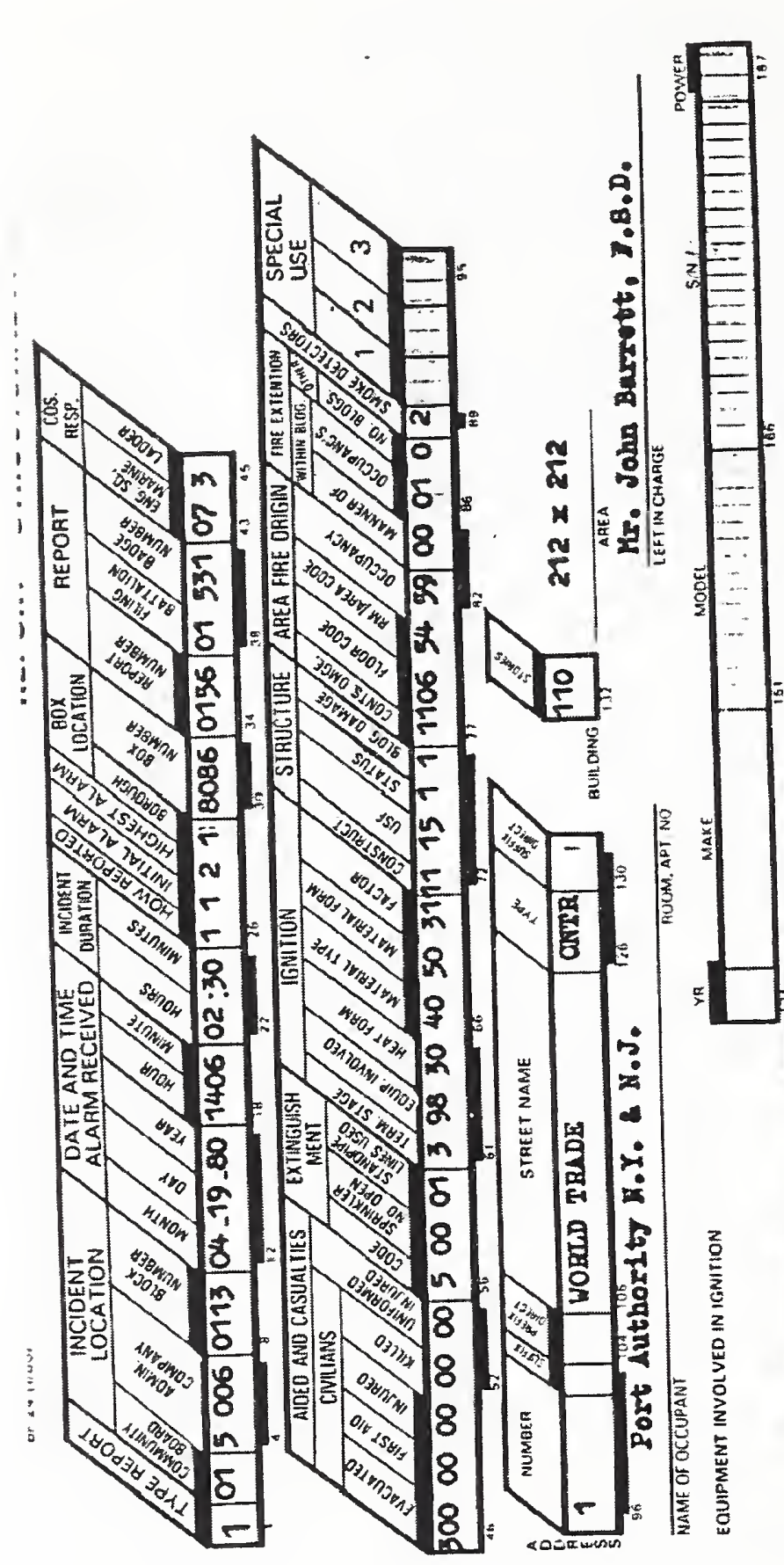

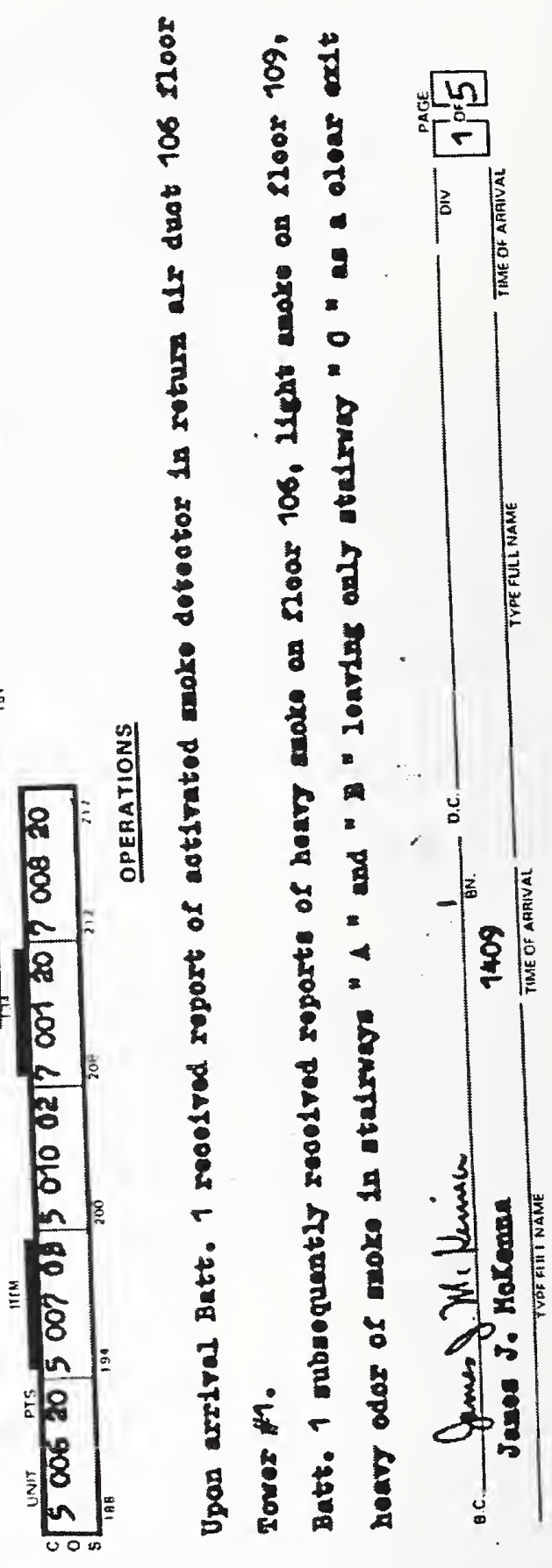

ADMINISTAATIVE COMPANY 


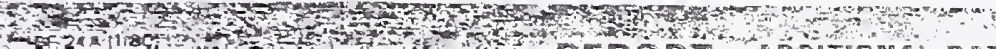

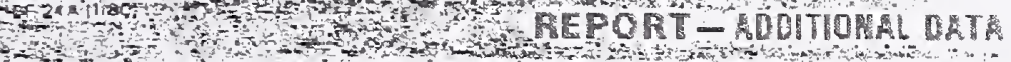

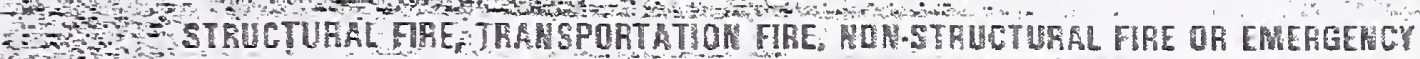

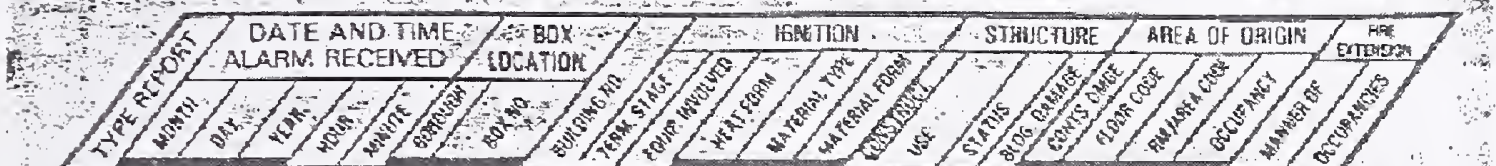

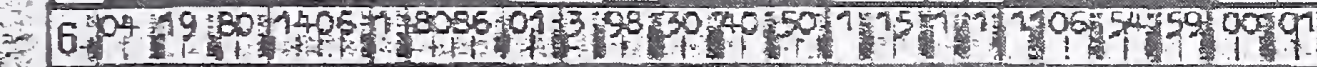

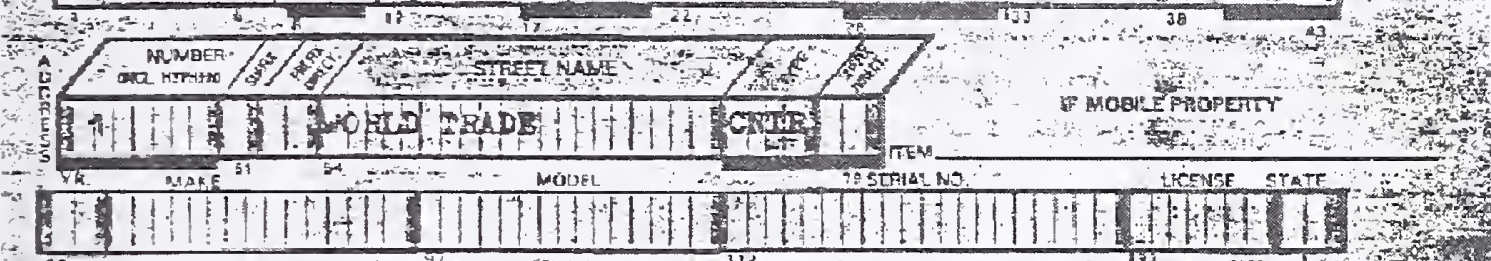

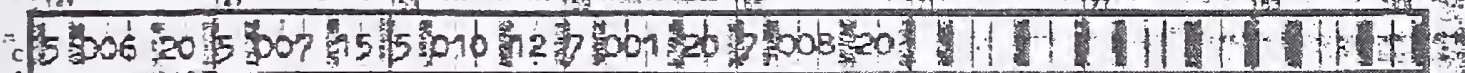

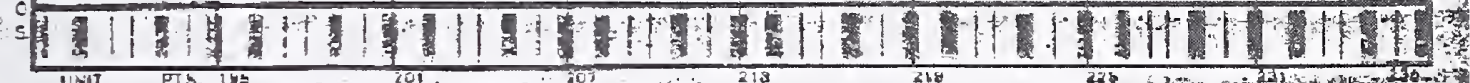

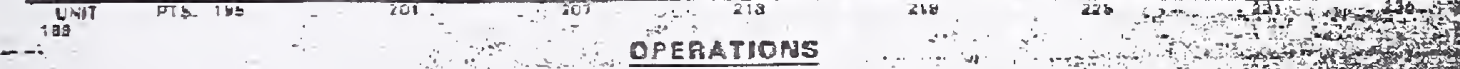

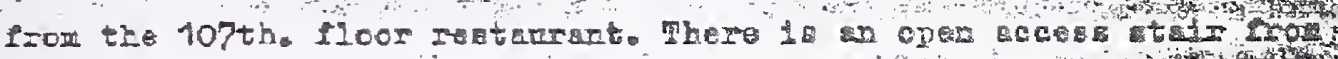

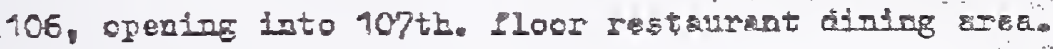

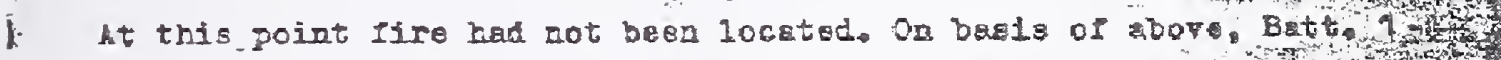
crtered approx. 300 pereons evacuated from the thindow on the voxld

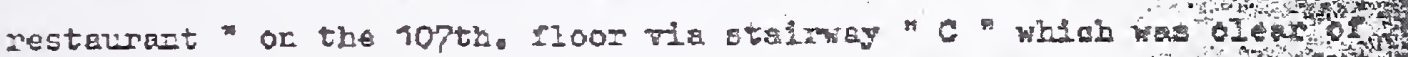

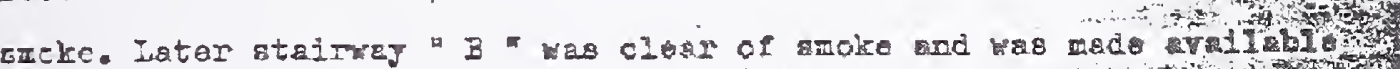
Ler evacuation.

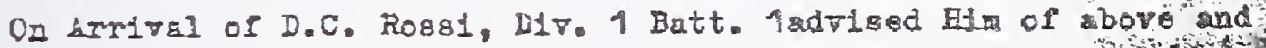

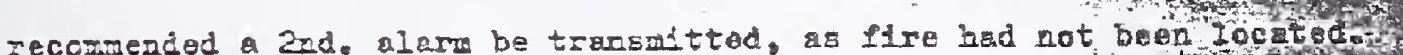

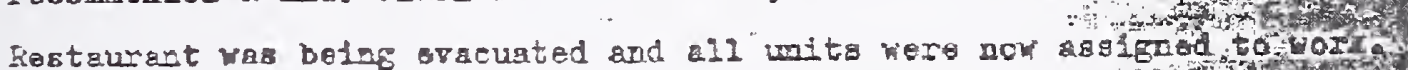

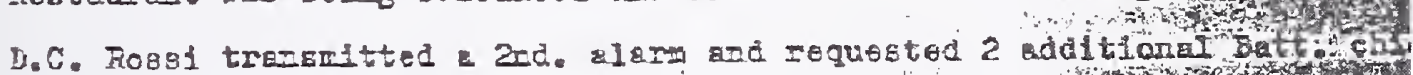
Operetions of Cos. ars us follows:

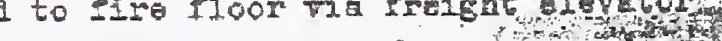

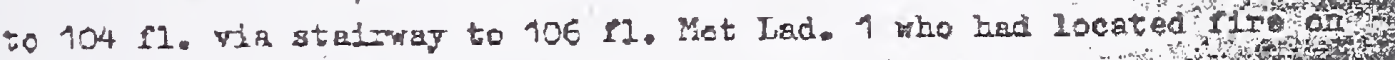

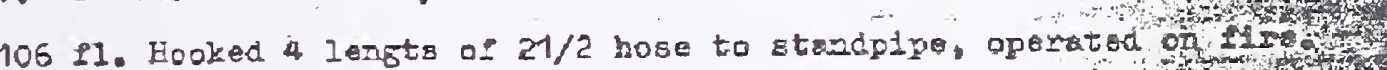

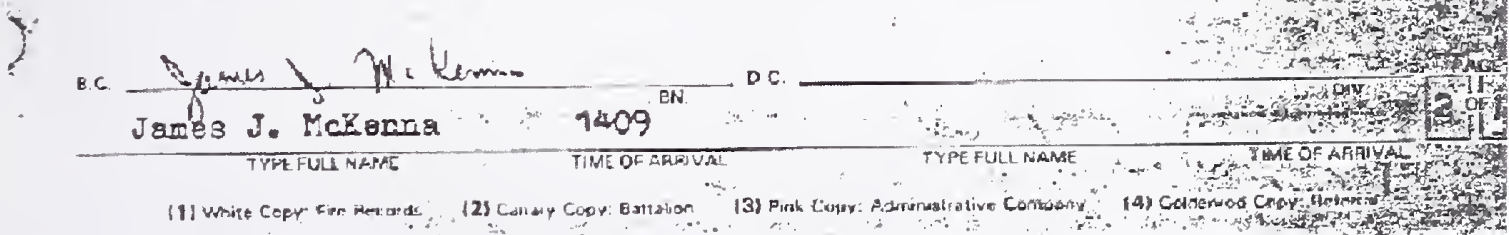




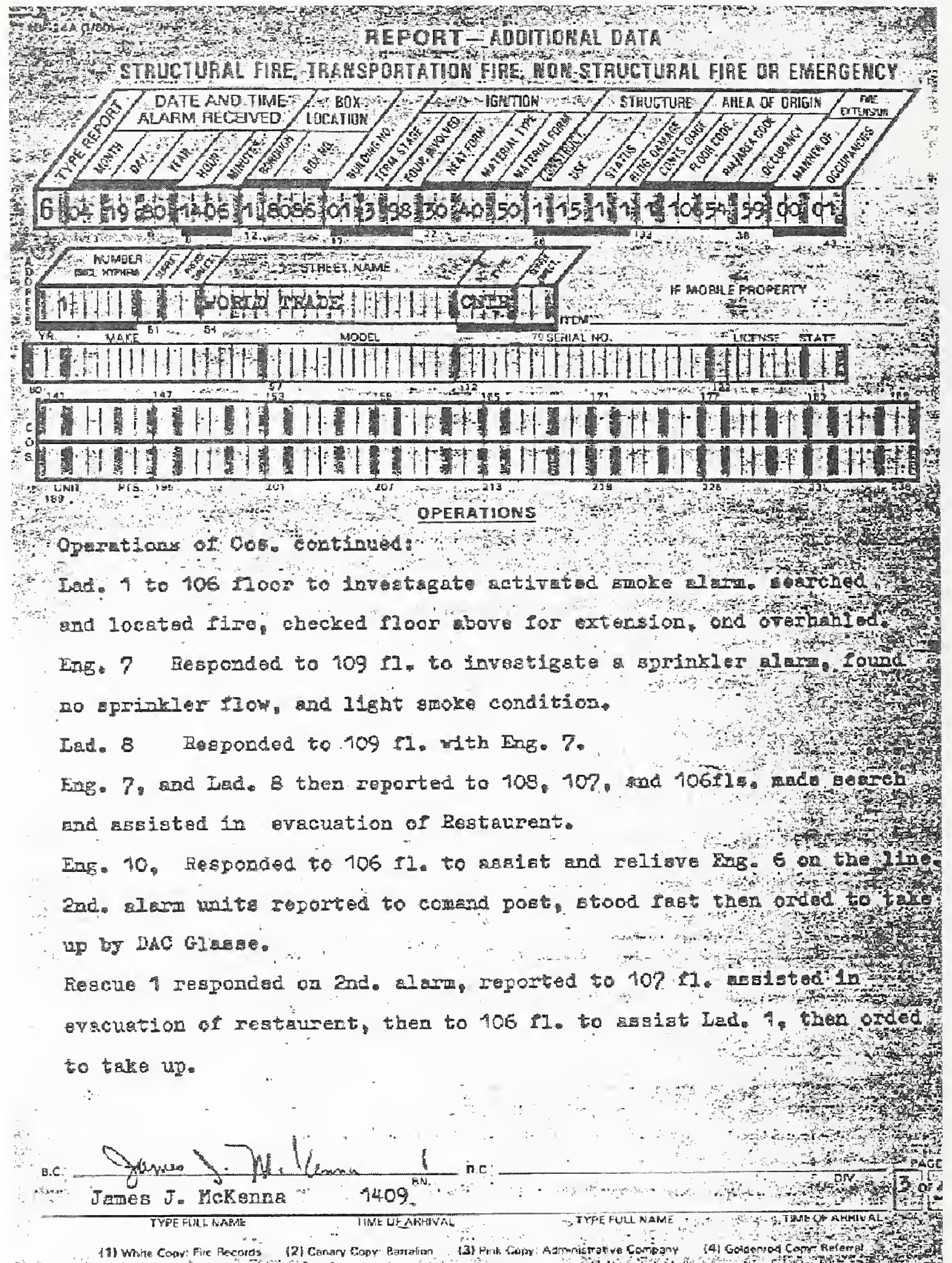

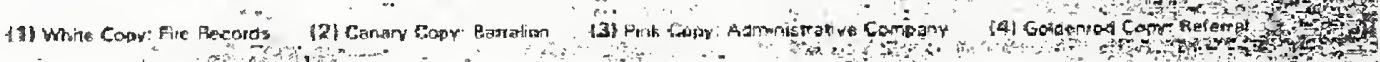




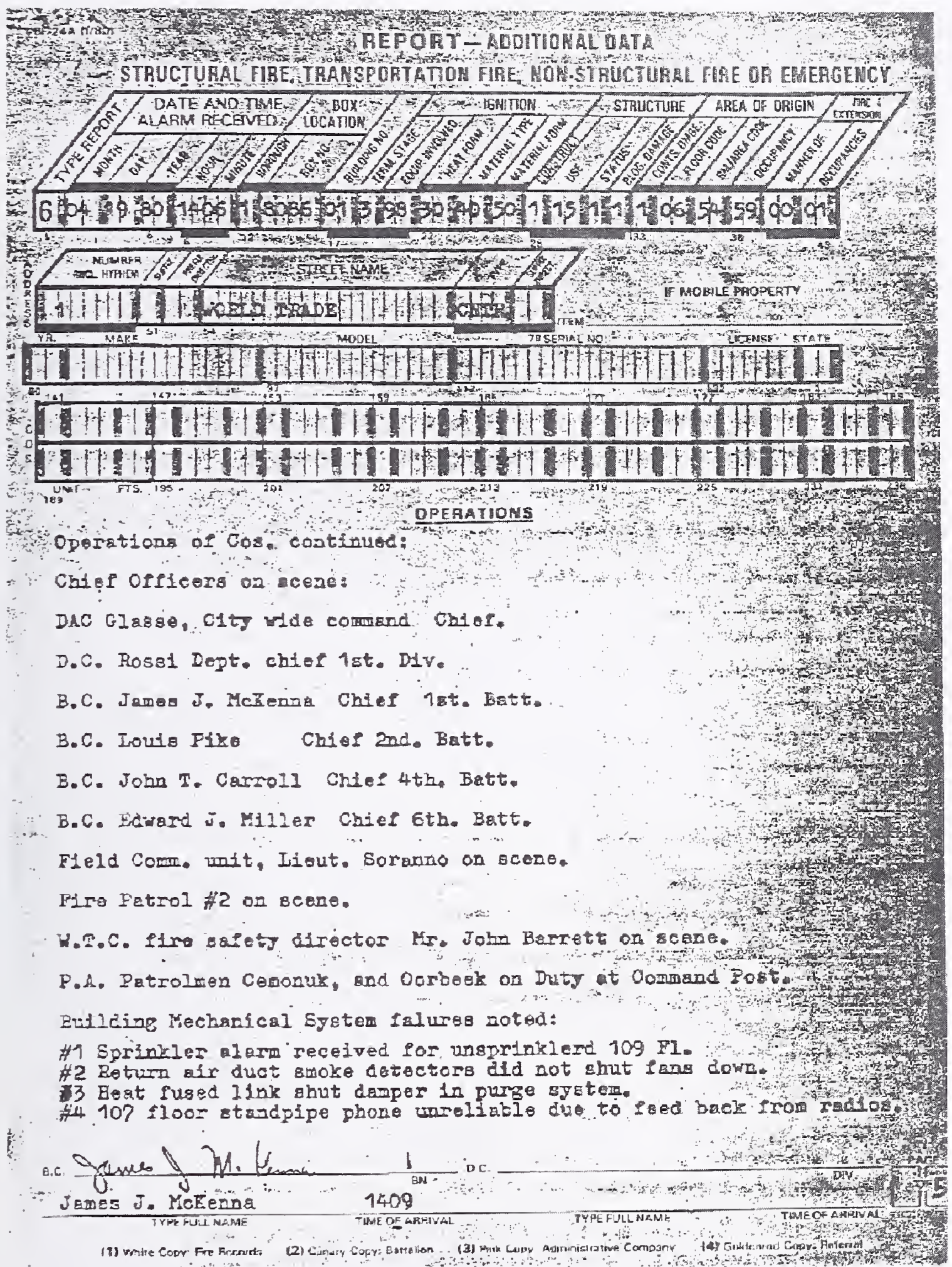




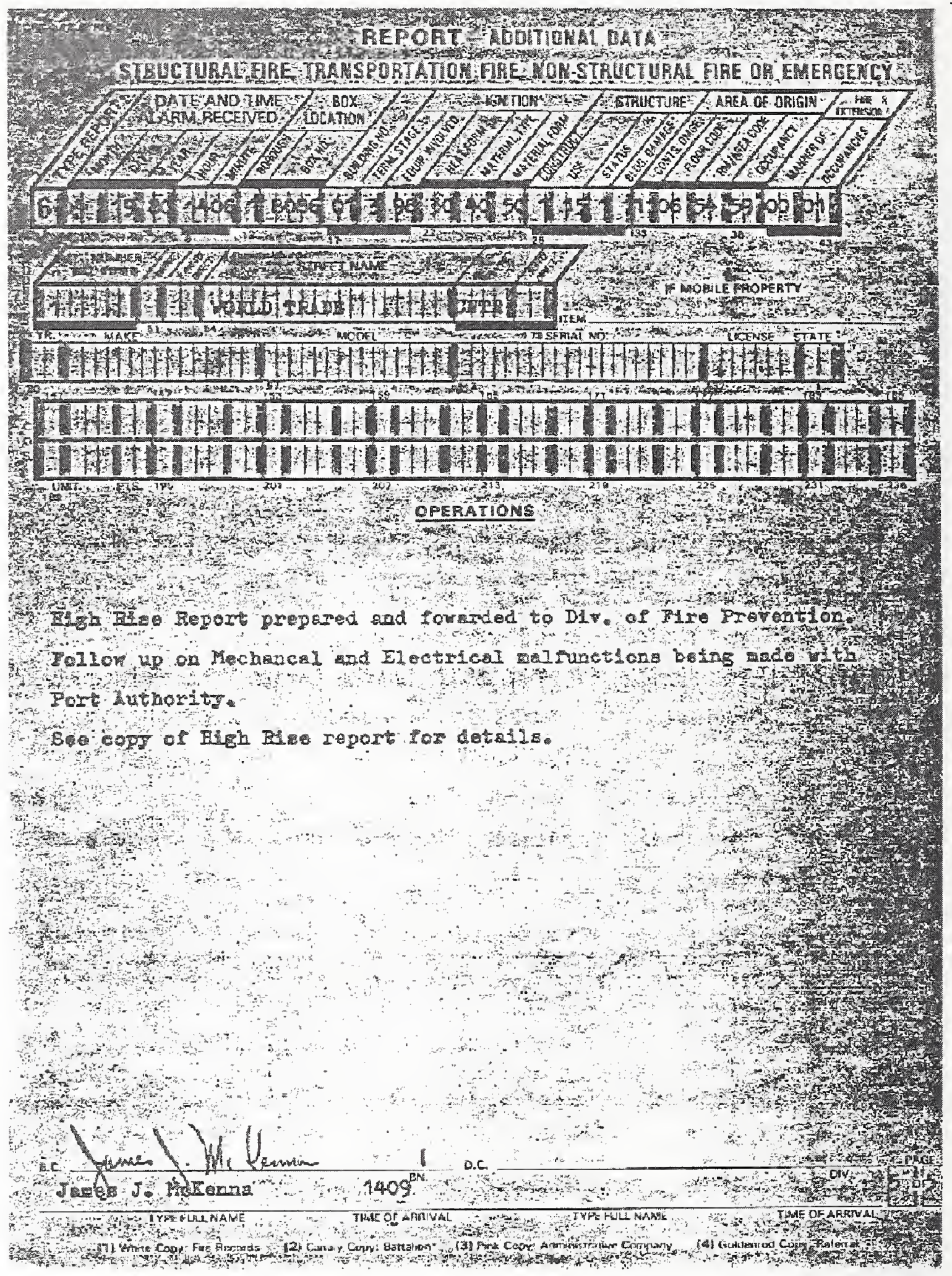



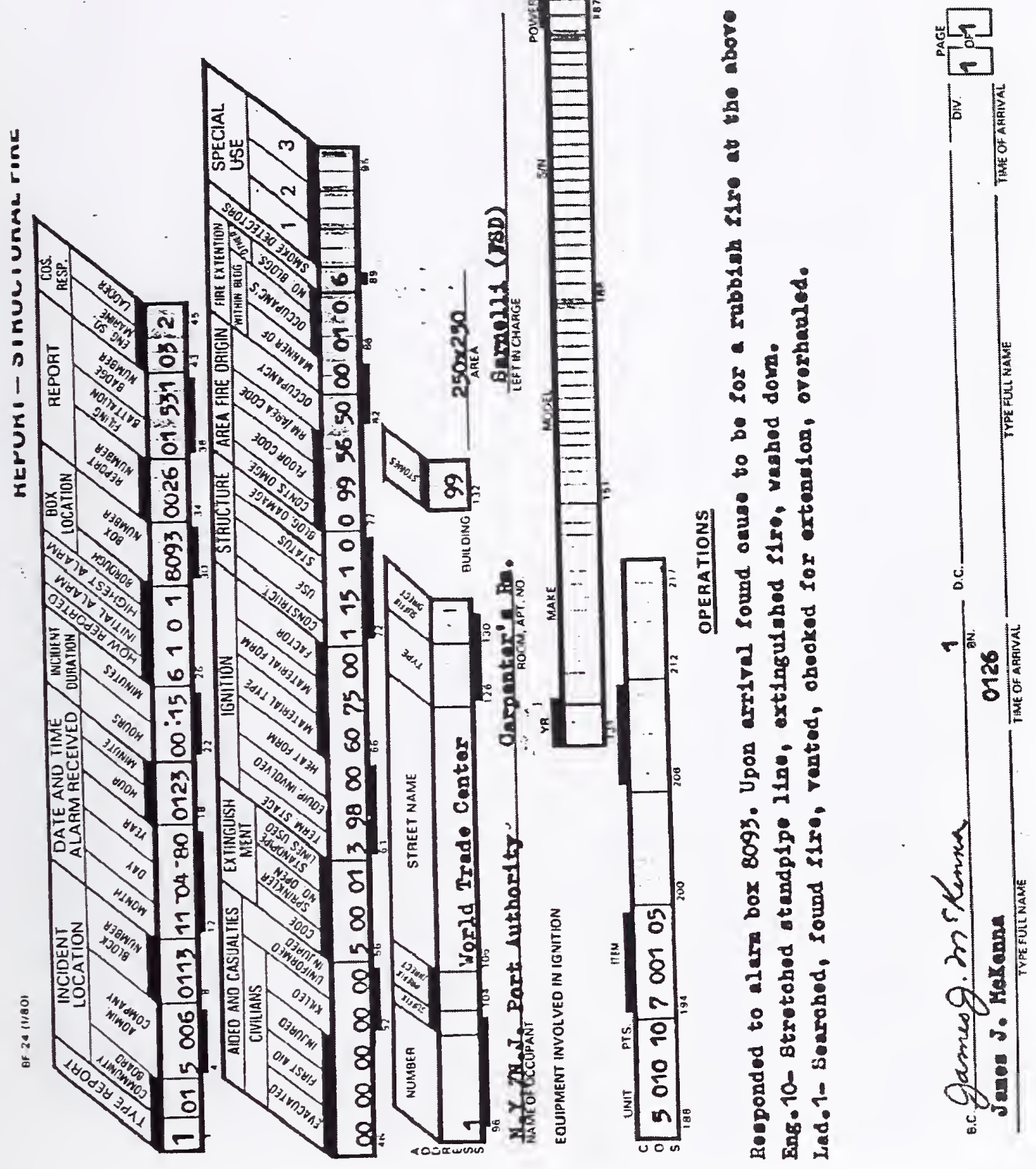

ADMINISTRATIVE COMPANY 


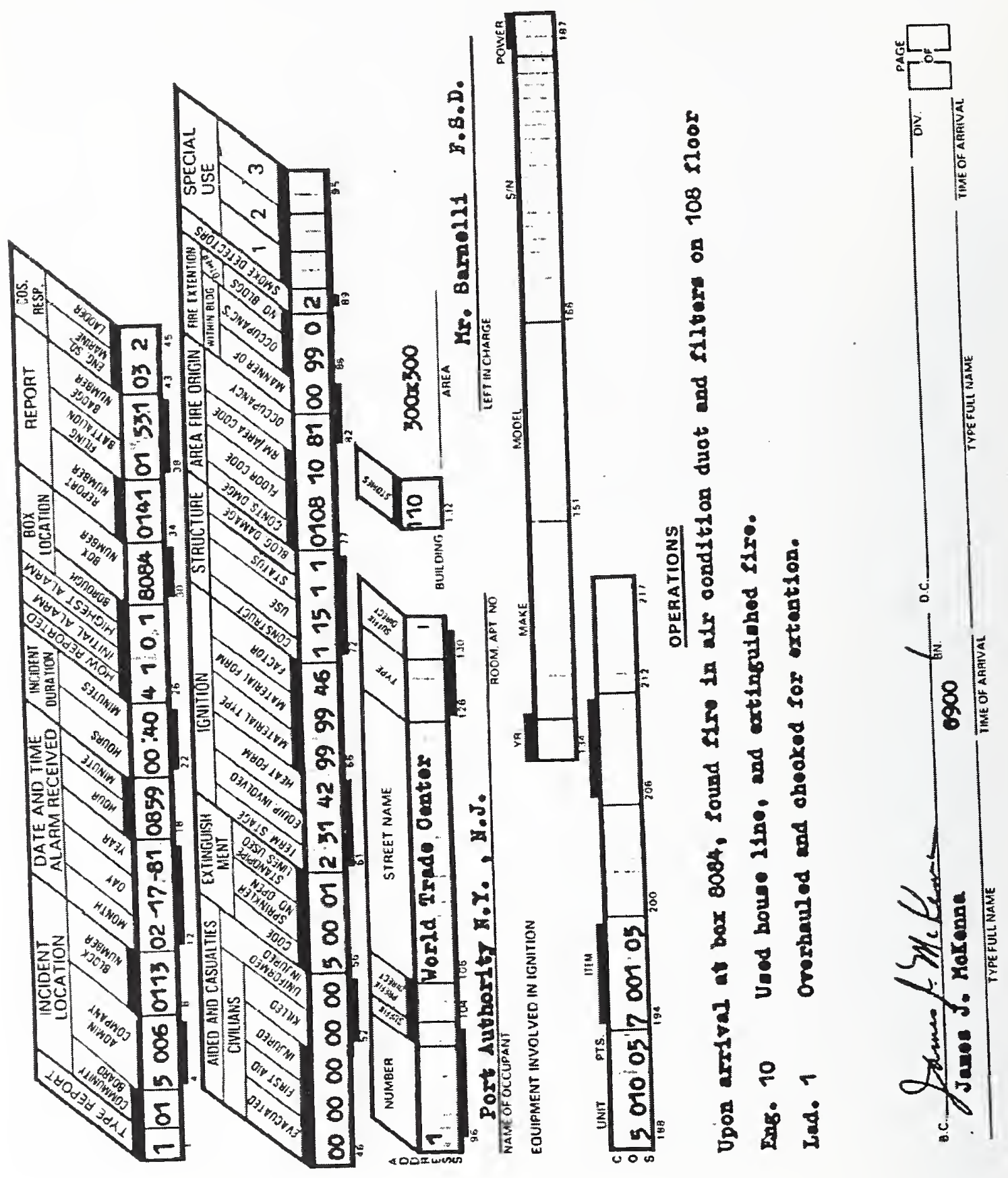



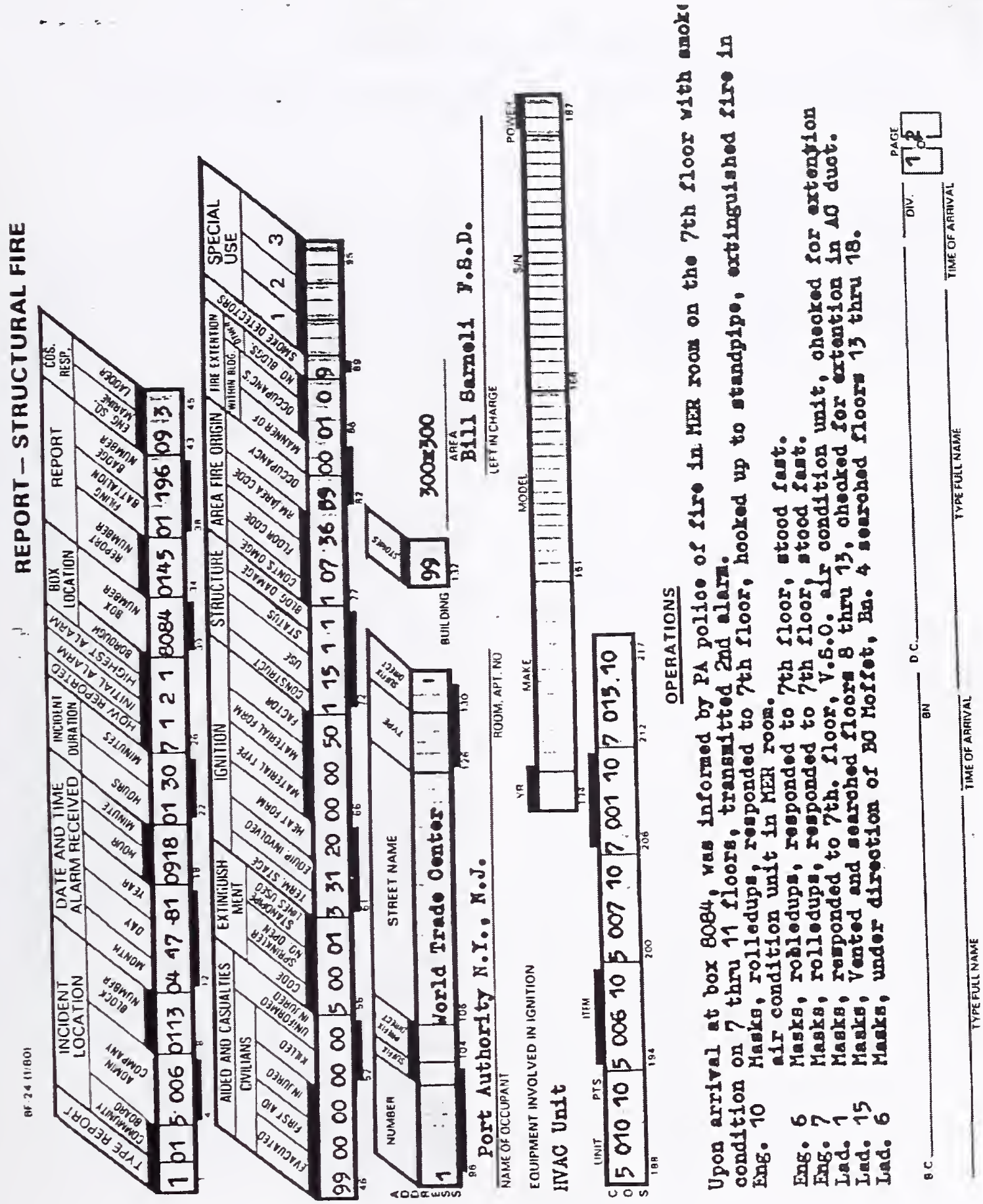


\section{REPORT - Additional Data}

Structural Fire, Transportation Fire, Non-Structural Fire or Emergency
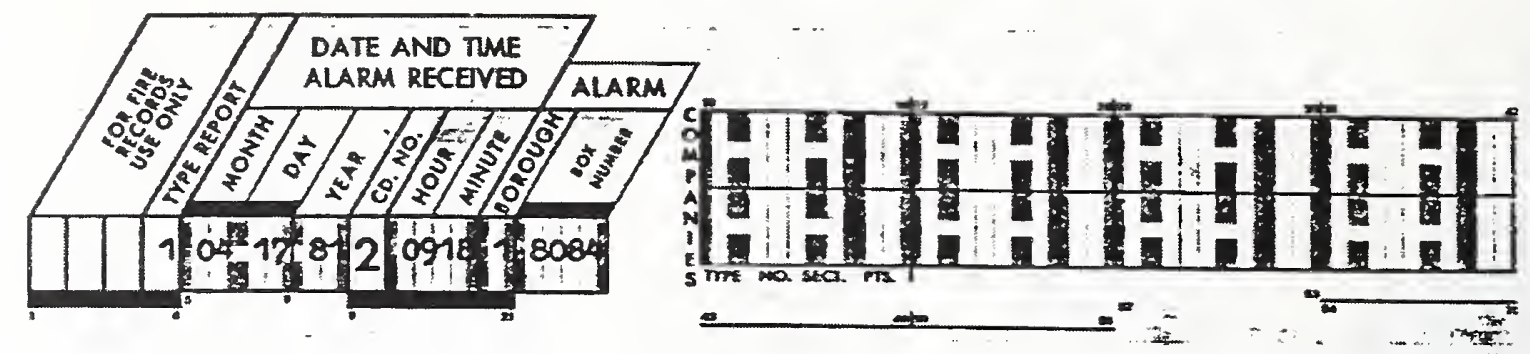

\section{OPERATIONS}

Bescue 1 Masks, soarched Noors 18 thru 23.

2nd. Im unita:

Engs. 24, 15, 55,9, 241, and Marine 1 vere orded to stand last at there coslened locations.

Div. 1. D.C. DeCaprio responded and superrised opperations

Chiels Gorrlez, Bishop, and Parrell also on sceno.

P.A. personell reported evacuation of aprax, 1,500 persons from noor 9 thra 23.

P.C. unit on scene

F.P. 2 on sceos.

TIYIES

Bor 0918

$\begin{array}{ll}2-2 & 0928 \\ 0 / C & 0959\end{array}$

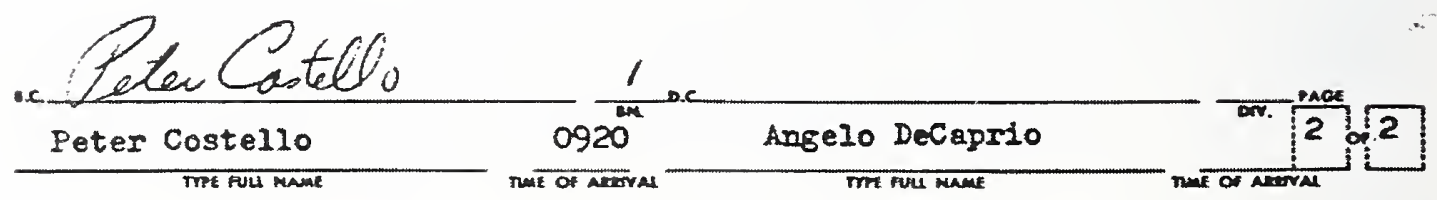




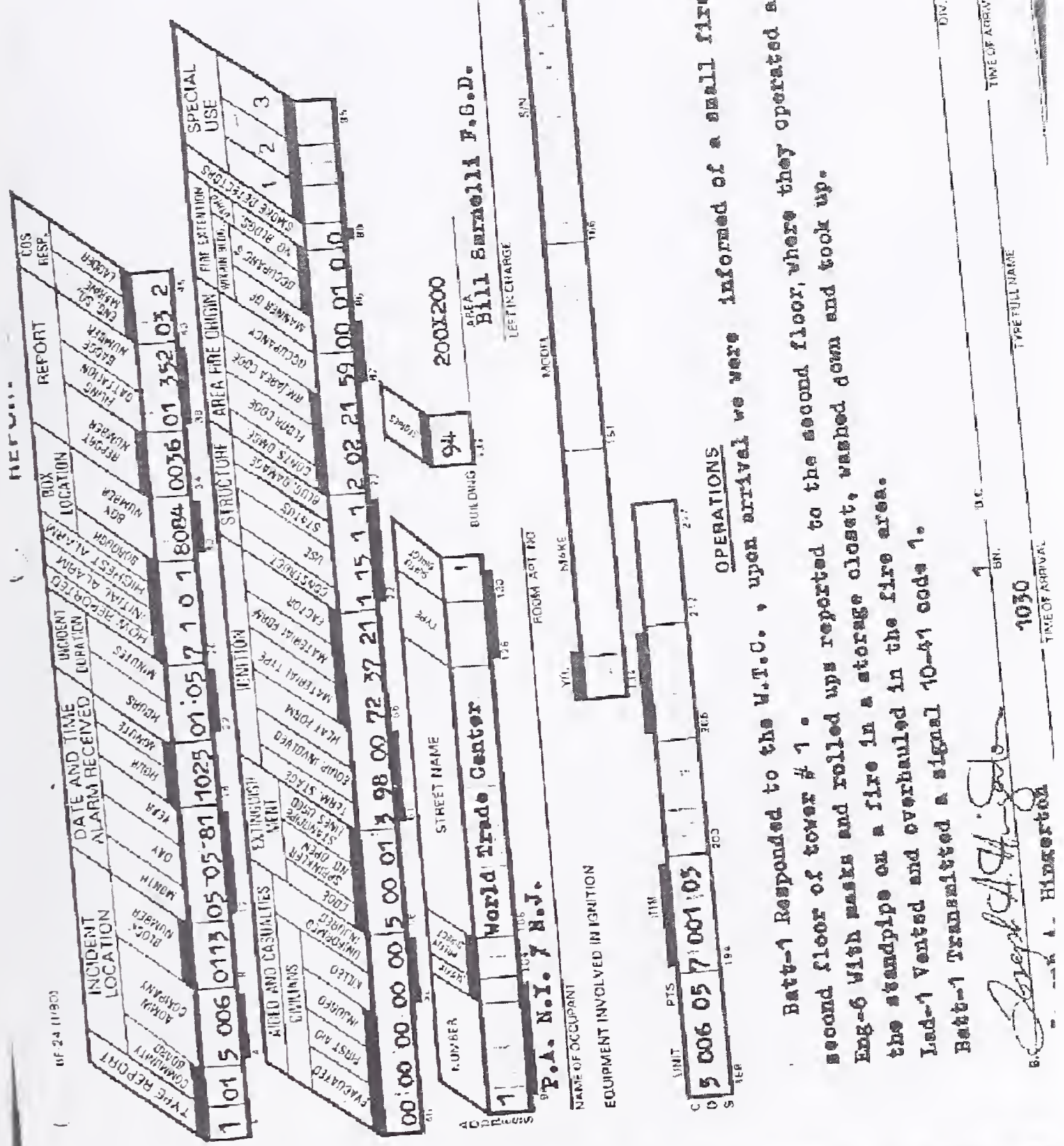




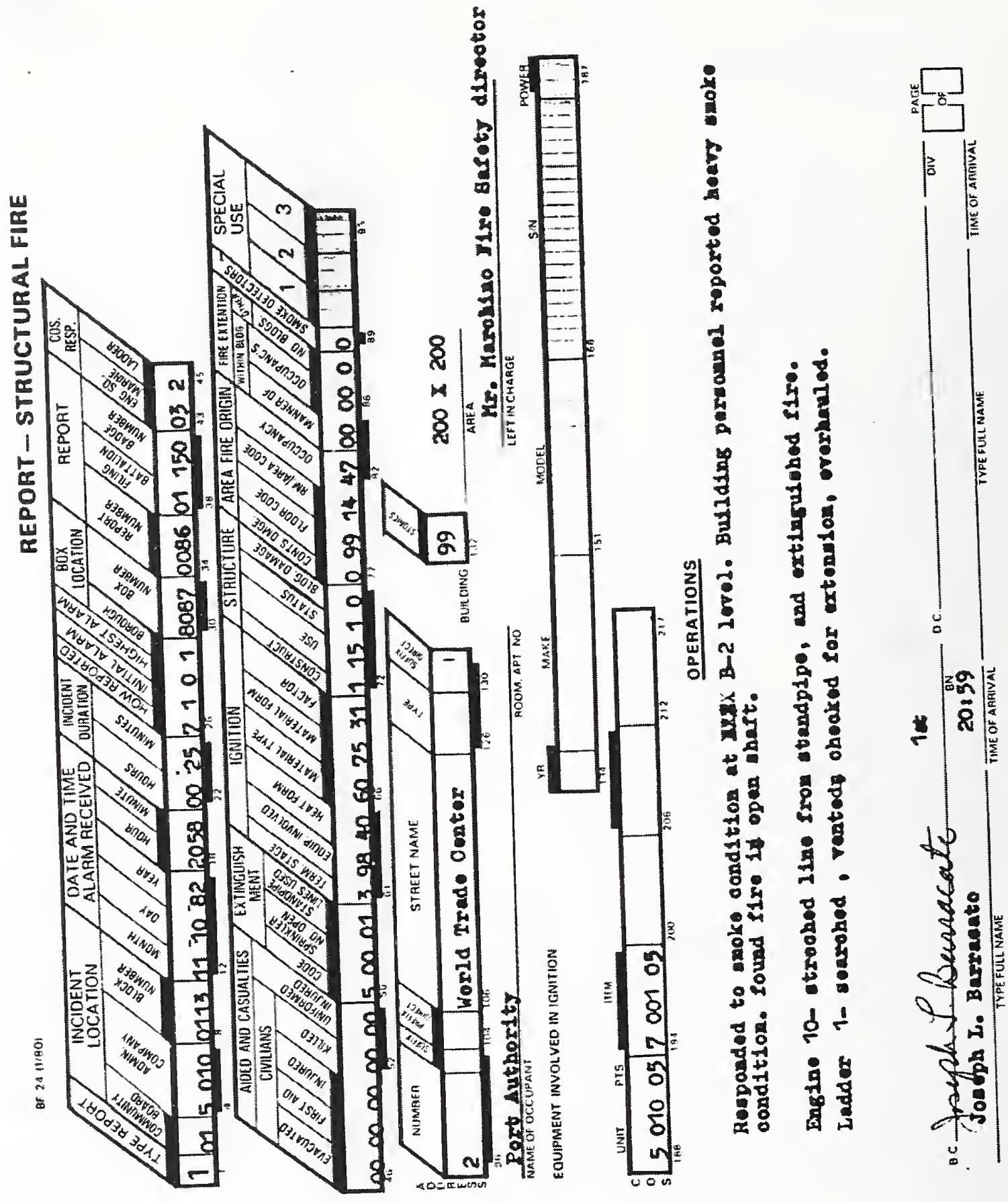




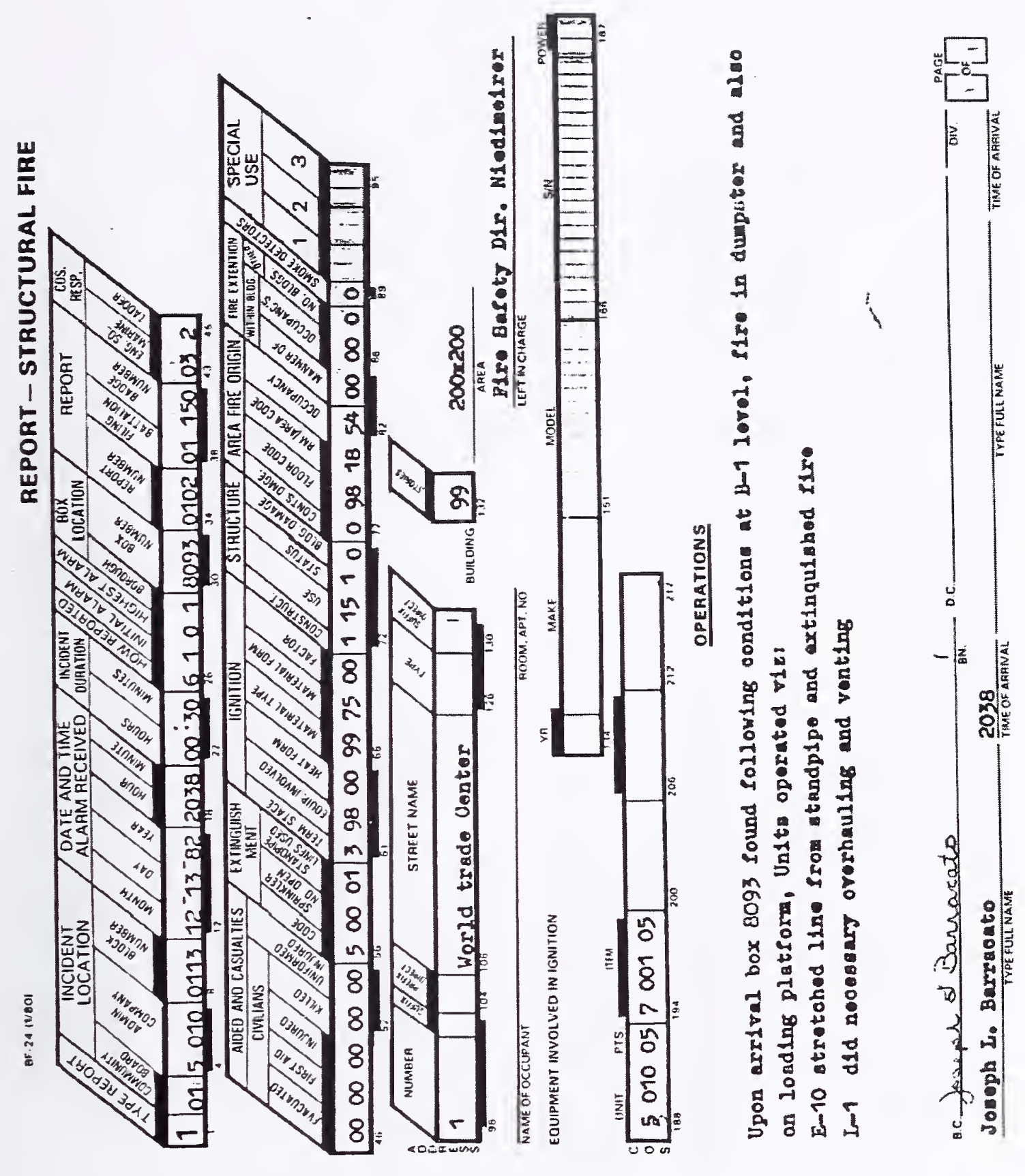




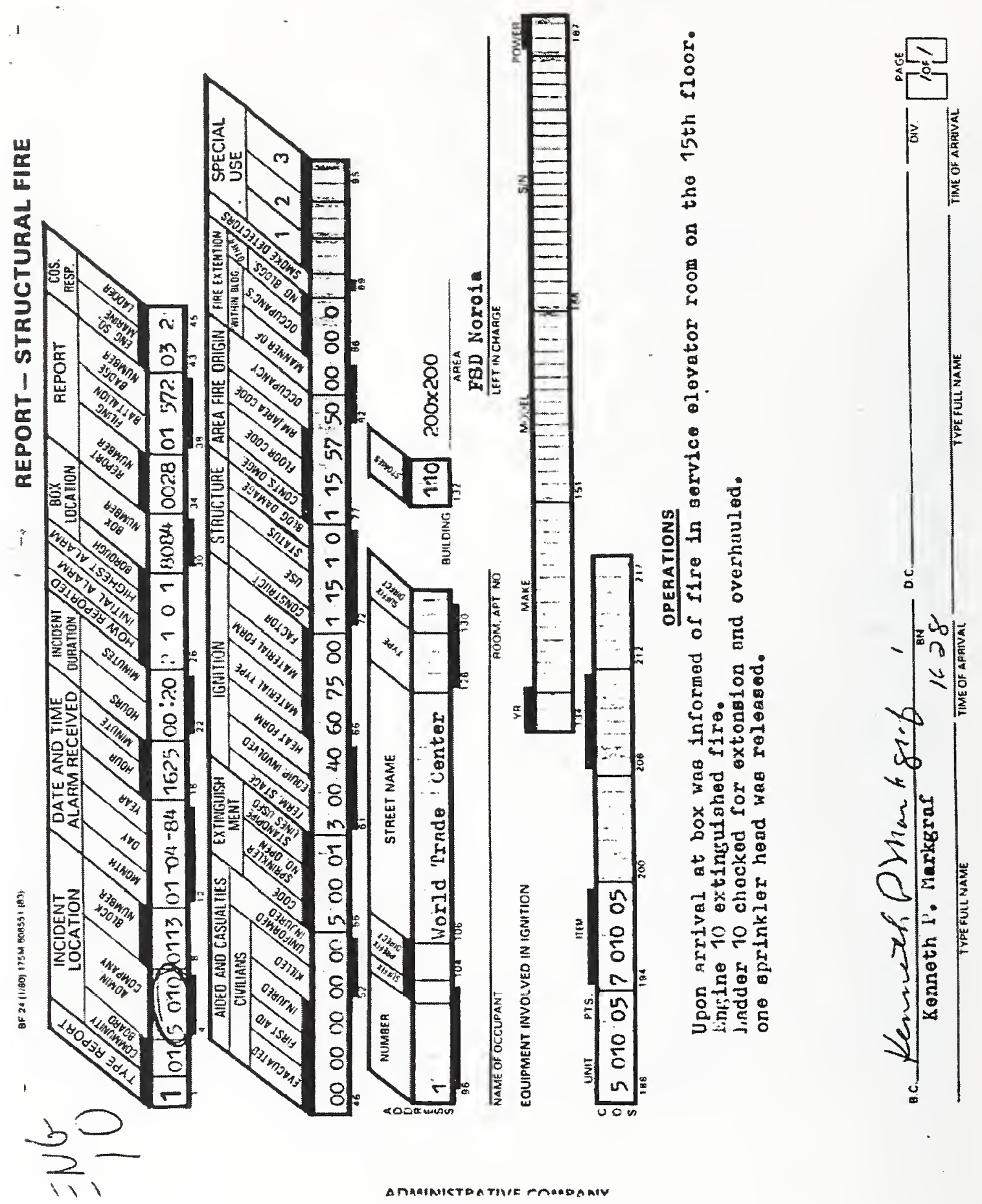




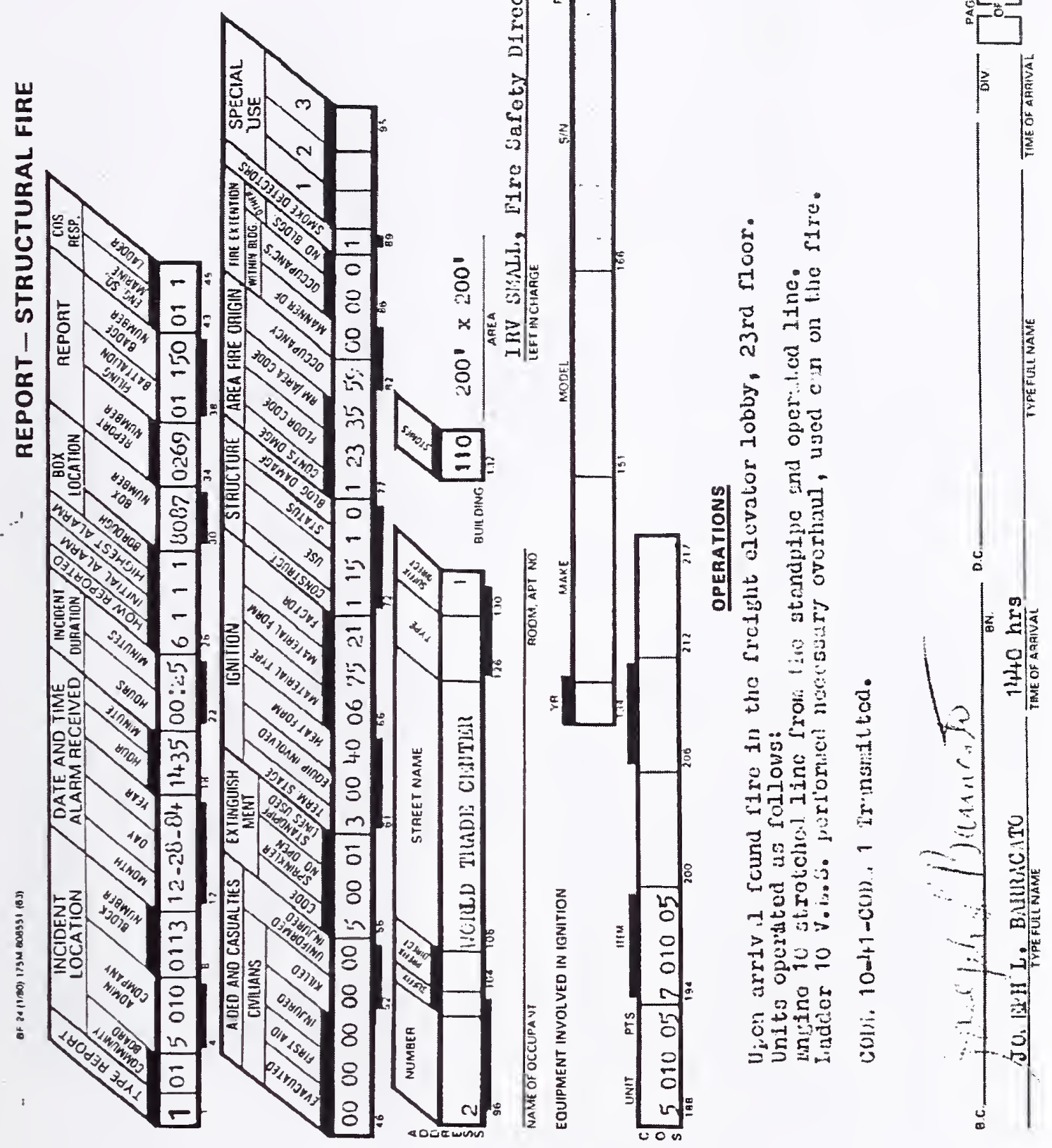




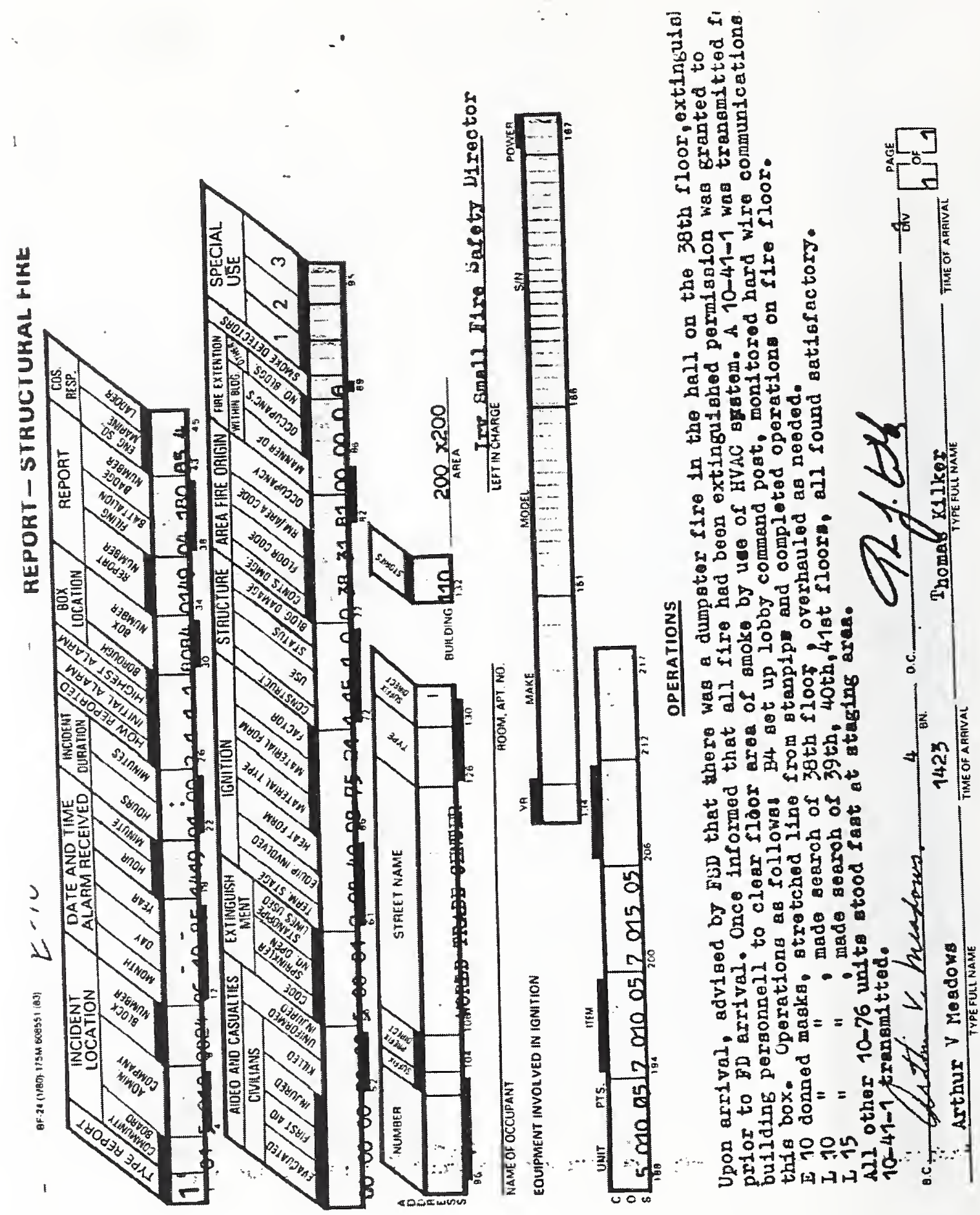




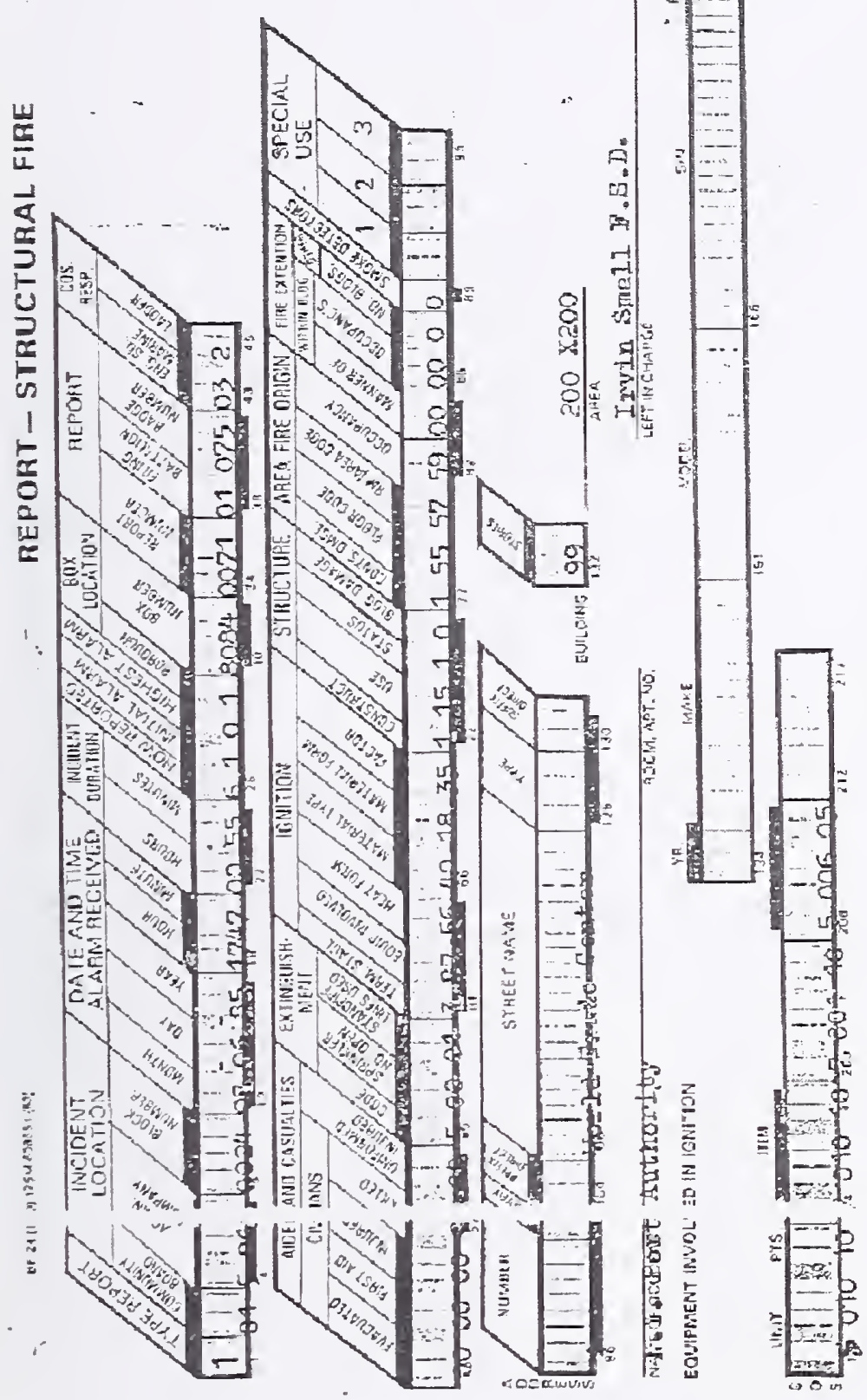

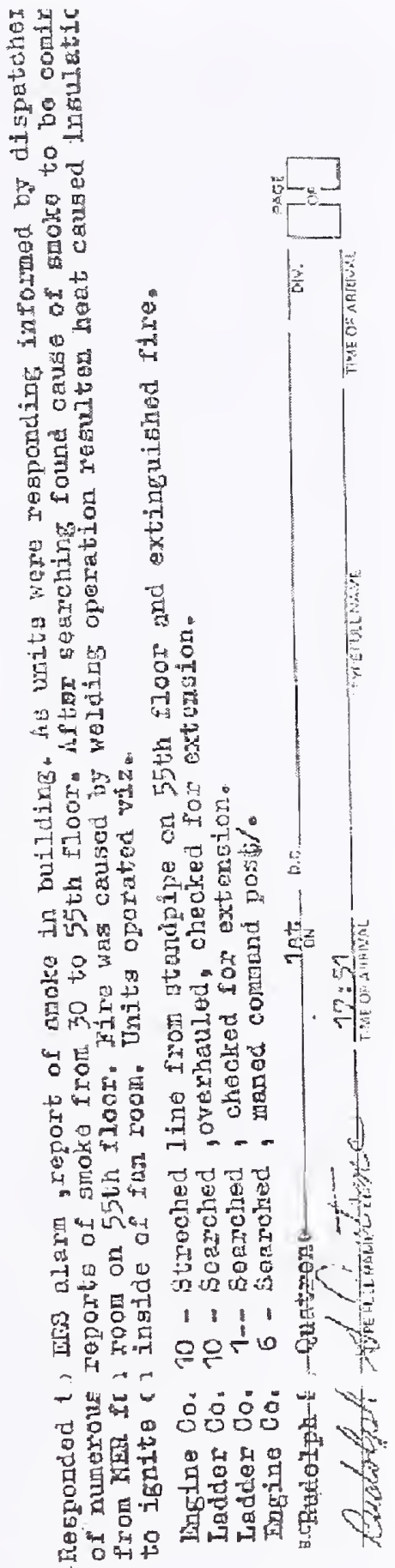




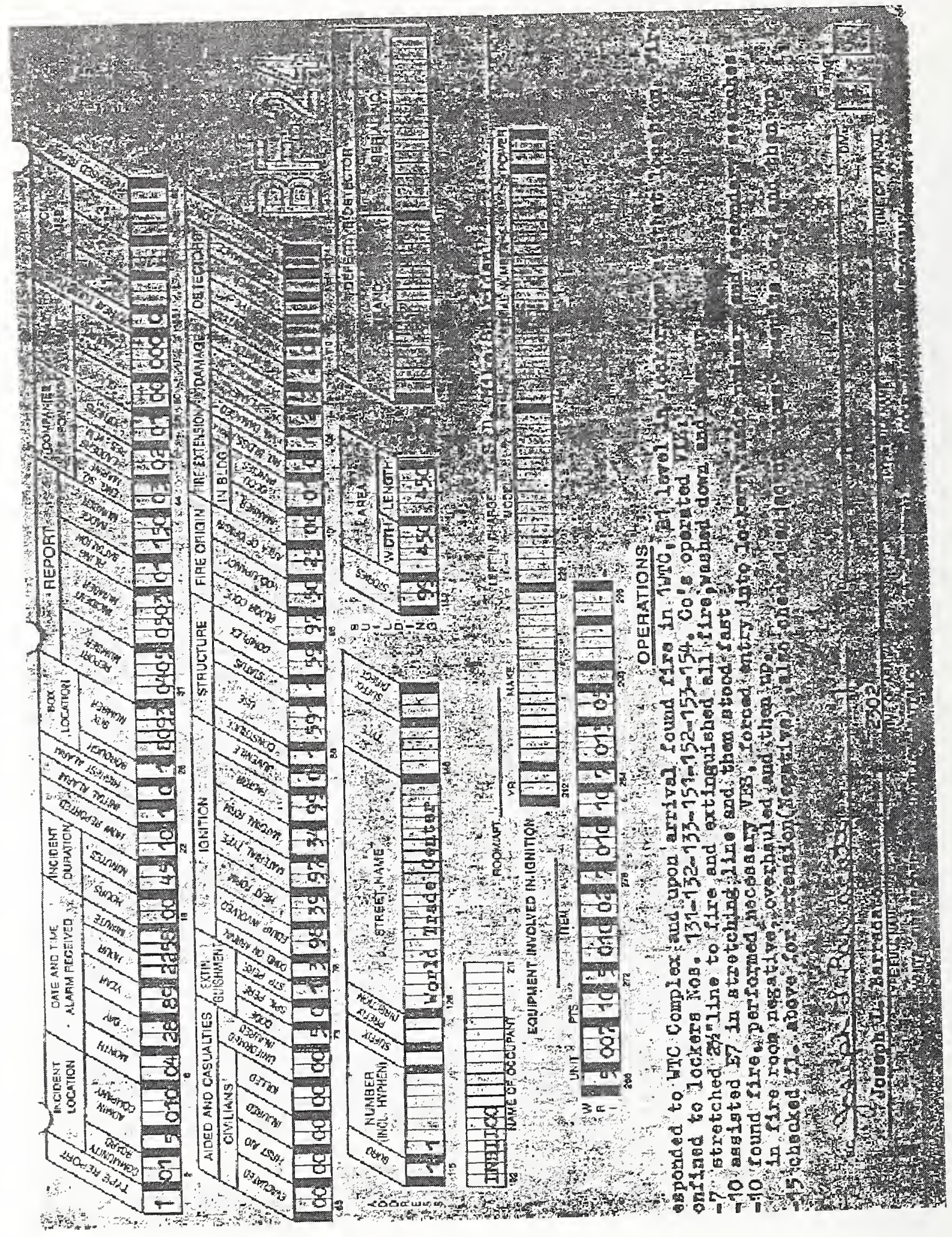




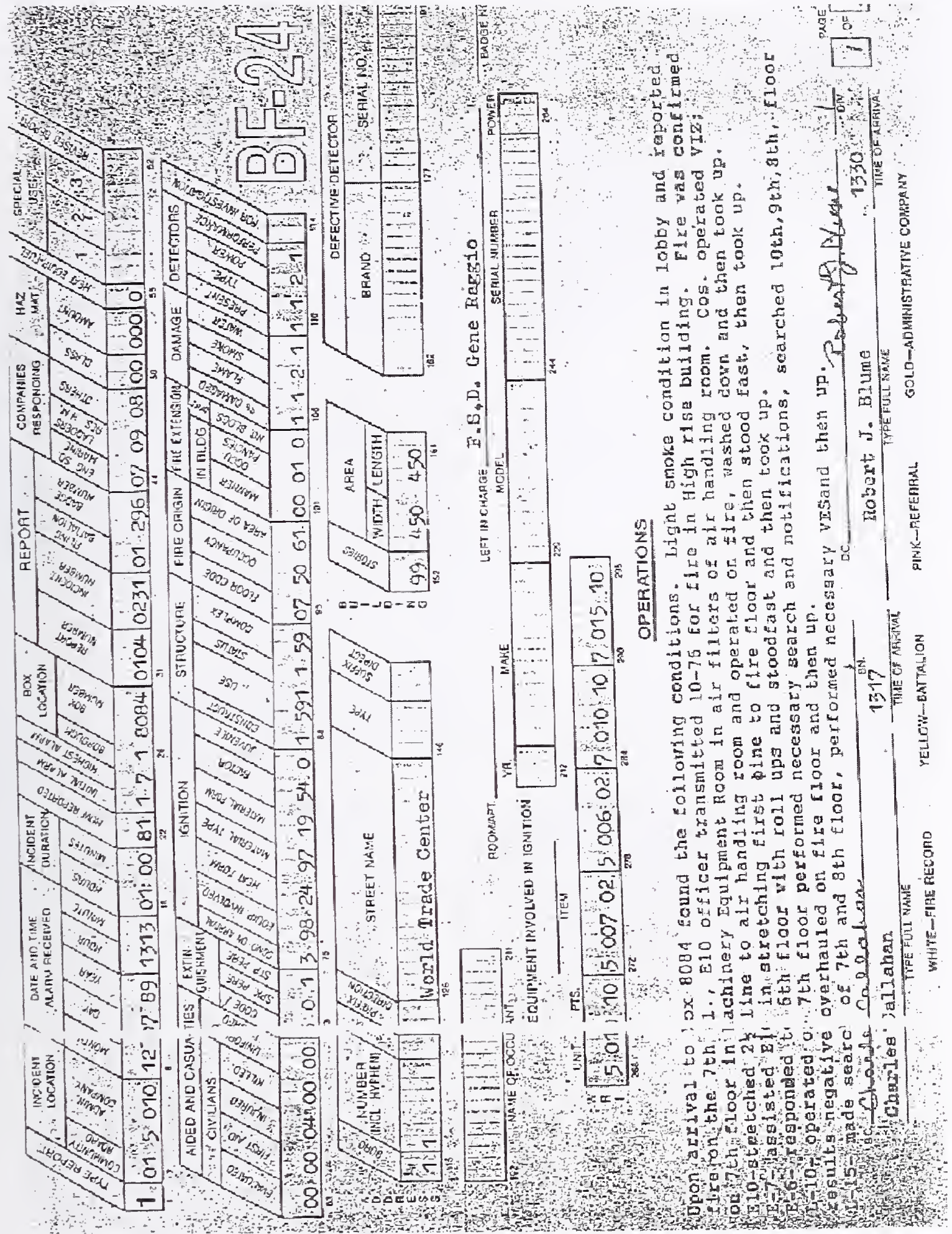




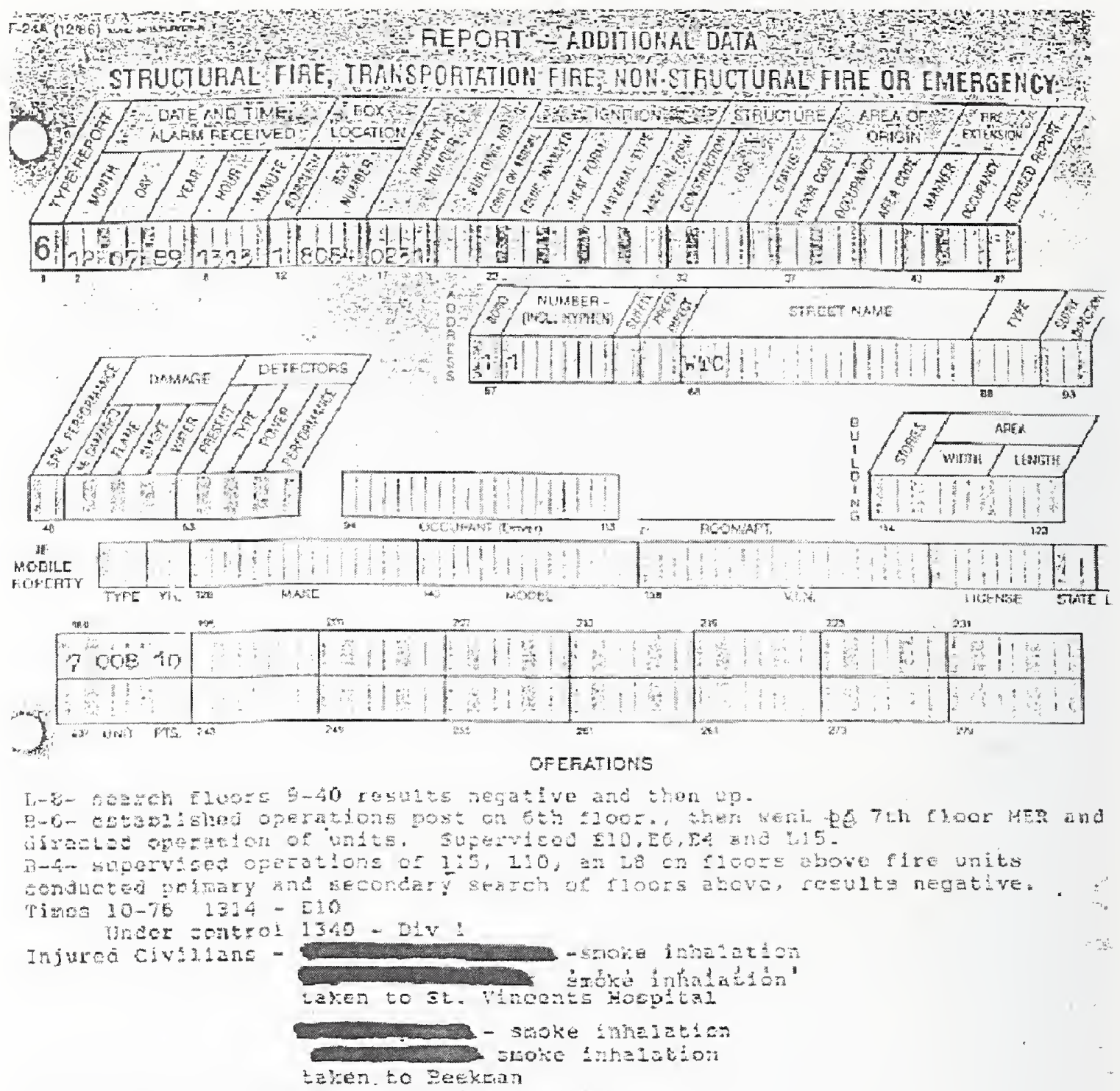

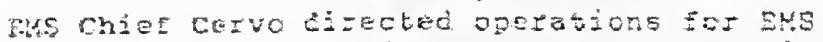

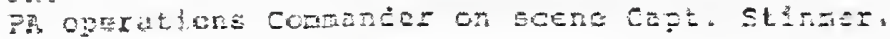

in

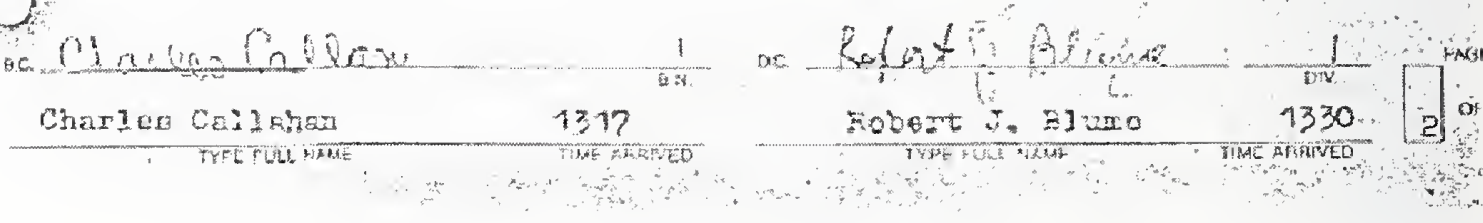




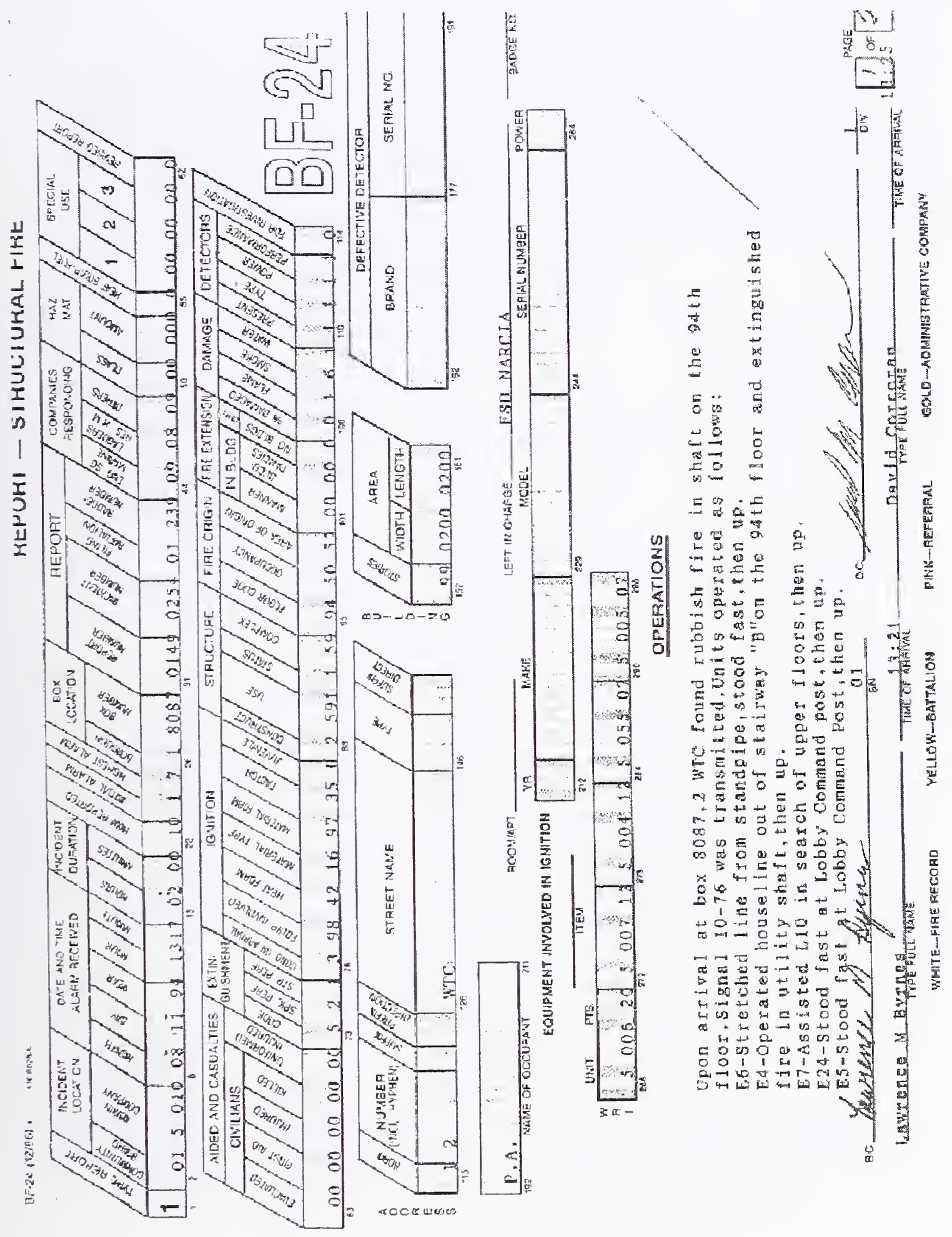



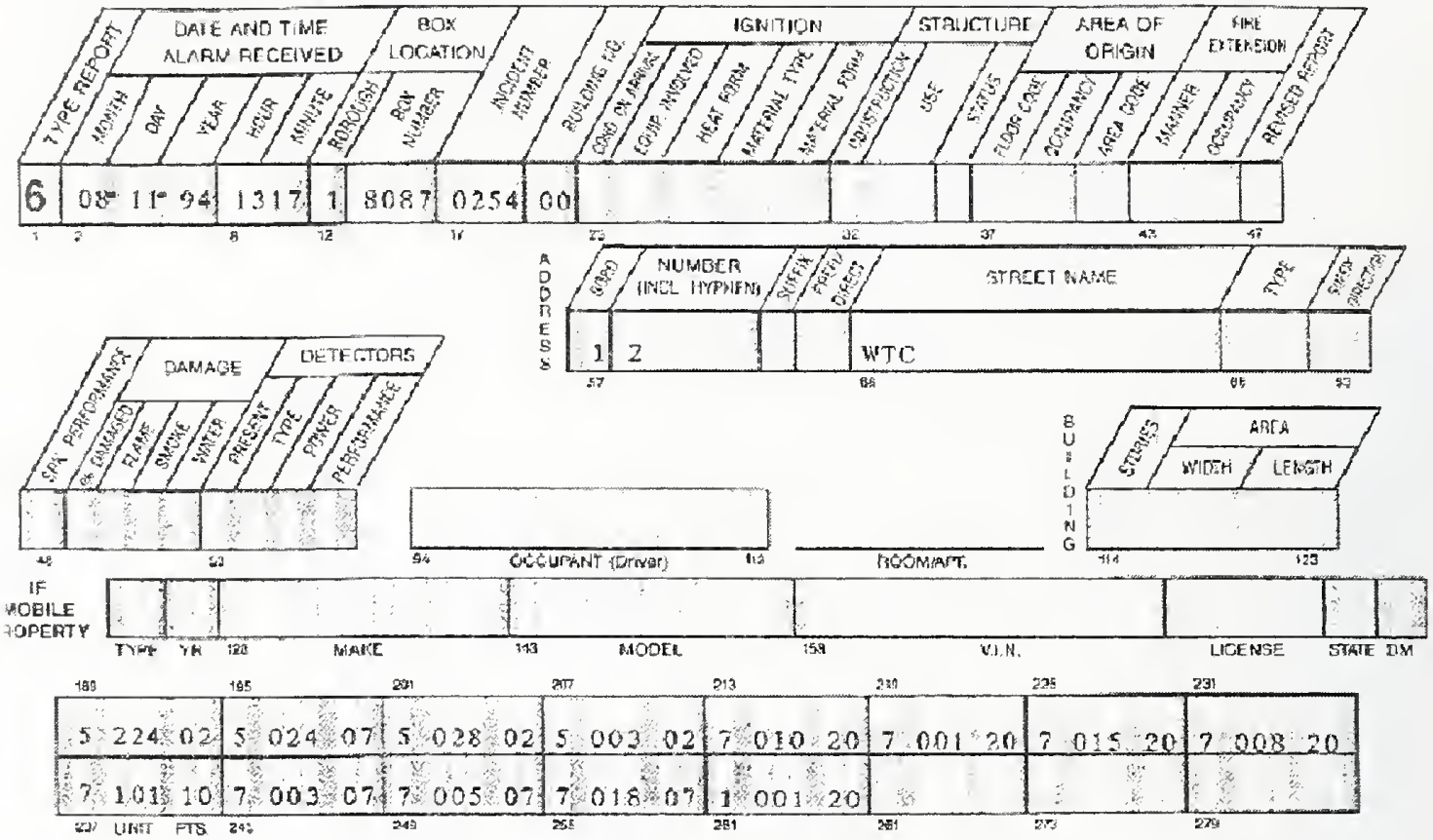

\section{OPERATIONS}

B3-5tood fist at the lobby comand post, then up.

E5s-stoul fast at lubhy command post, then up.

I. I - poerated on the 107th floor oponed various walls and doors, finding no

fire or extentjon, then up.

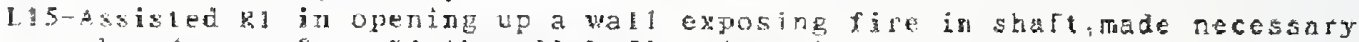
search and exar from y4 thru 3 th floorlezmation point of

shaft) Nercative.

L8-Made necegary search and exam of lloors 100 ihlu 106 , found negatine.

1.5-5tcod fast at the loboy Command Fos b, then up.

LI $8-3$ tood fast at the lobby canmere post, then up.

Livi-sedsh 75,91 and sind floor, found negative, ilien us?.

1.1-Made necessary seatch and cxam of floors $10 \mathrm{~A}$ thru log, then up.

L224-5tood fast at the Lobby Comand Post, then up.

R-bsed Therma!-Imging camera and located lire in shat on the 9 th ploot.

Bat th-supervised operation on the fire floot, then up.

Batt8-Supervised operations on 107 and 74 floors, then wp.

but $12-5$ tood fast, then up.

FCU-Monilored handi-talkie circuit, porotess reporte, then up. A
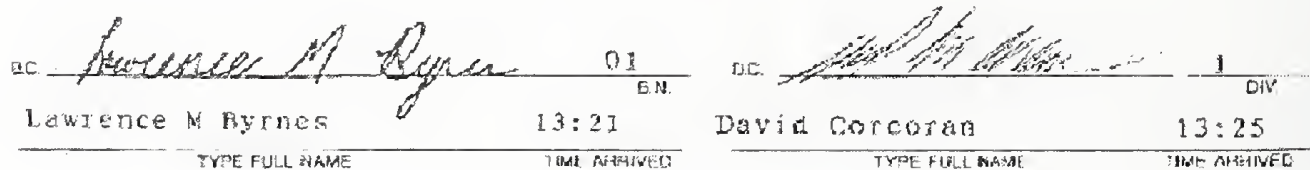


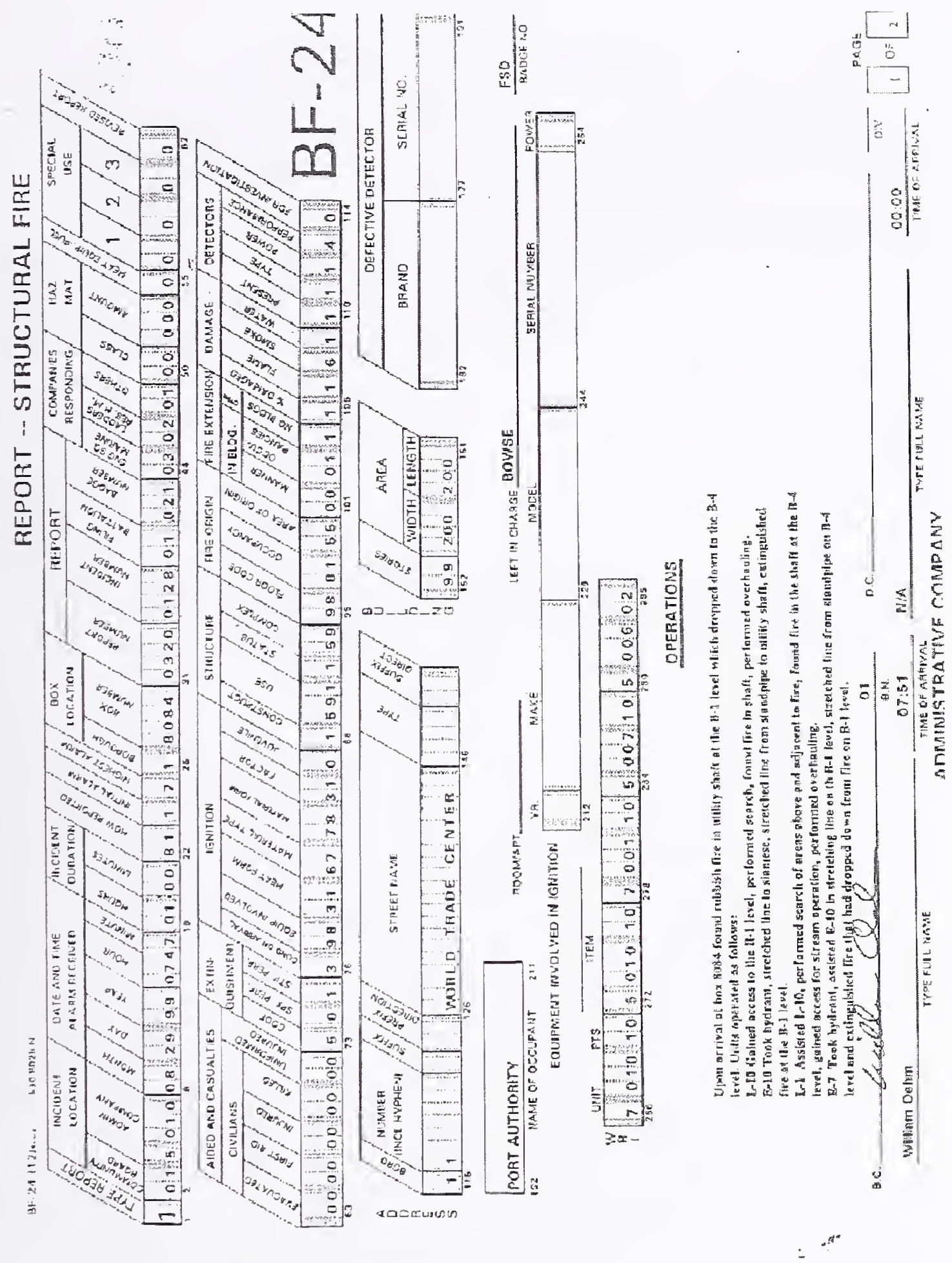




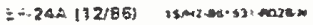

REPORT - ADDITIONAL DATA

STRUCTURAL FIRE, TRANSPORTATION FIRE, NON-STRUCTURAL FIRE OR EMERGENCY
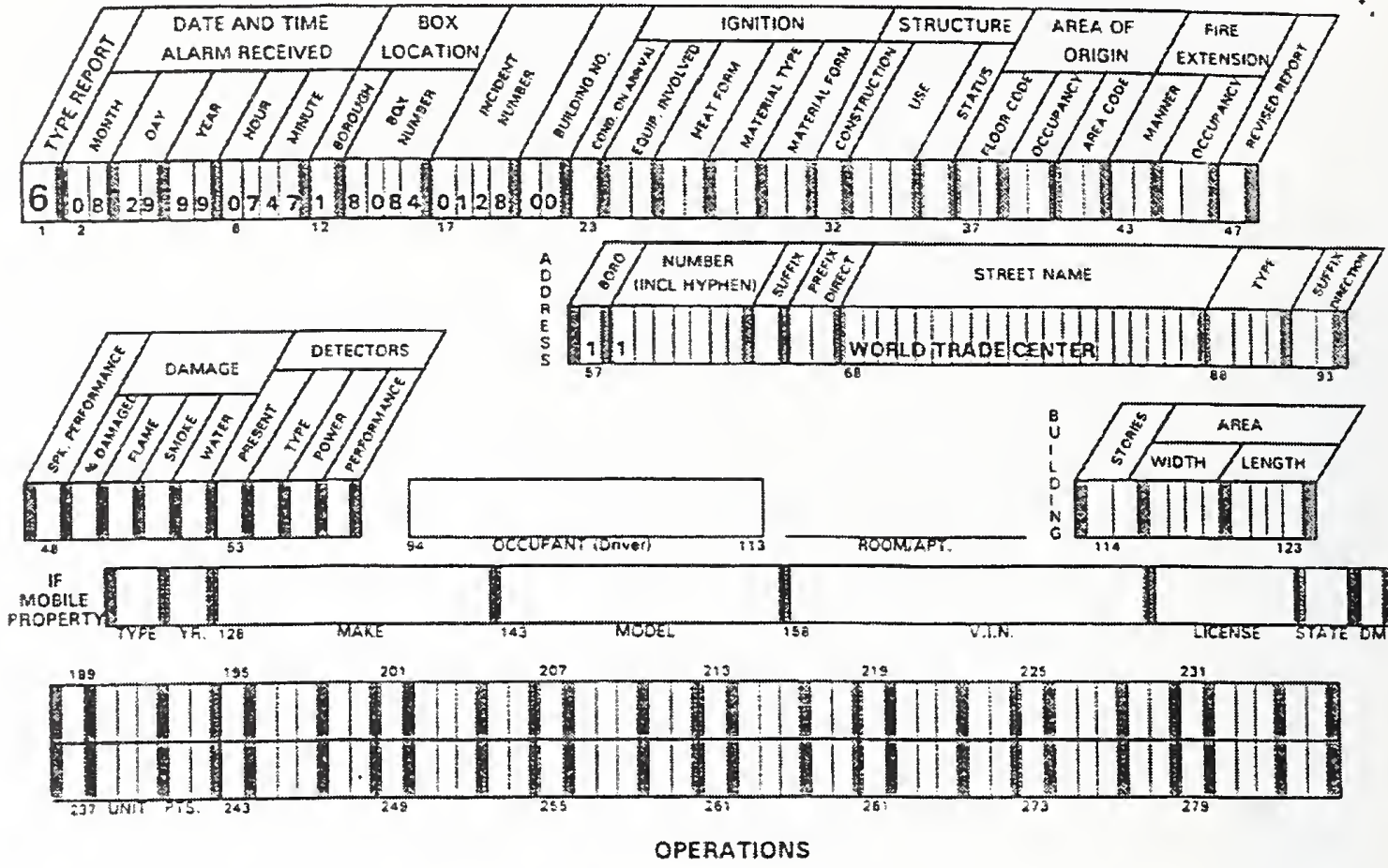

E-6 Took bydrant, assisted $\mathrm{E}-7$ in stretch.
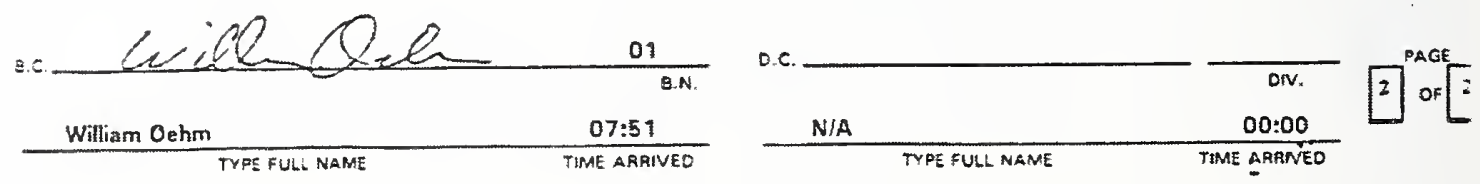


\section{Appendix H \\ SIGNIFICANT FIRES IN WTC 7}

Significant fires occurring in WTC 7

\begin{tabular}{|c|c|l|l|c|c|c|}
\hline $\begin{array}{c}\text { Significant } \\
\text { Fire }\end{array}$ & $\begin{array}{c}\text { Incident } \\
\text { Date }\end{array}$ & Fire Location & $\begin{array}{c}\text { \# sprinklers } \\
\text { activated }\end{array}$ & $\begin{array}{c}\text { \# standpipes } \\
\text { activated }\end{array}$ & Cause of fire & $\begin{array}{c}\text { Material } \\
\text { Ignited }\end{array}$ \\
\hline 1 & $5 / 20 / 88$ & $\begin{array}{l}\text { Construction } \\
\text { shanties on } \\
\text { floor } 3\end{array}$ & $\begin{array}{l}\text { Multiple, \# not } \\
\text { listed }\end{array}$ & 1 & Suspicious & Shanties \\
\hline
\end{tabular}




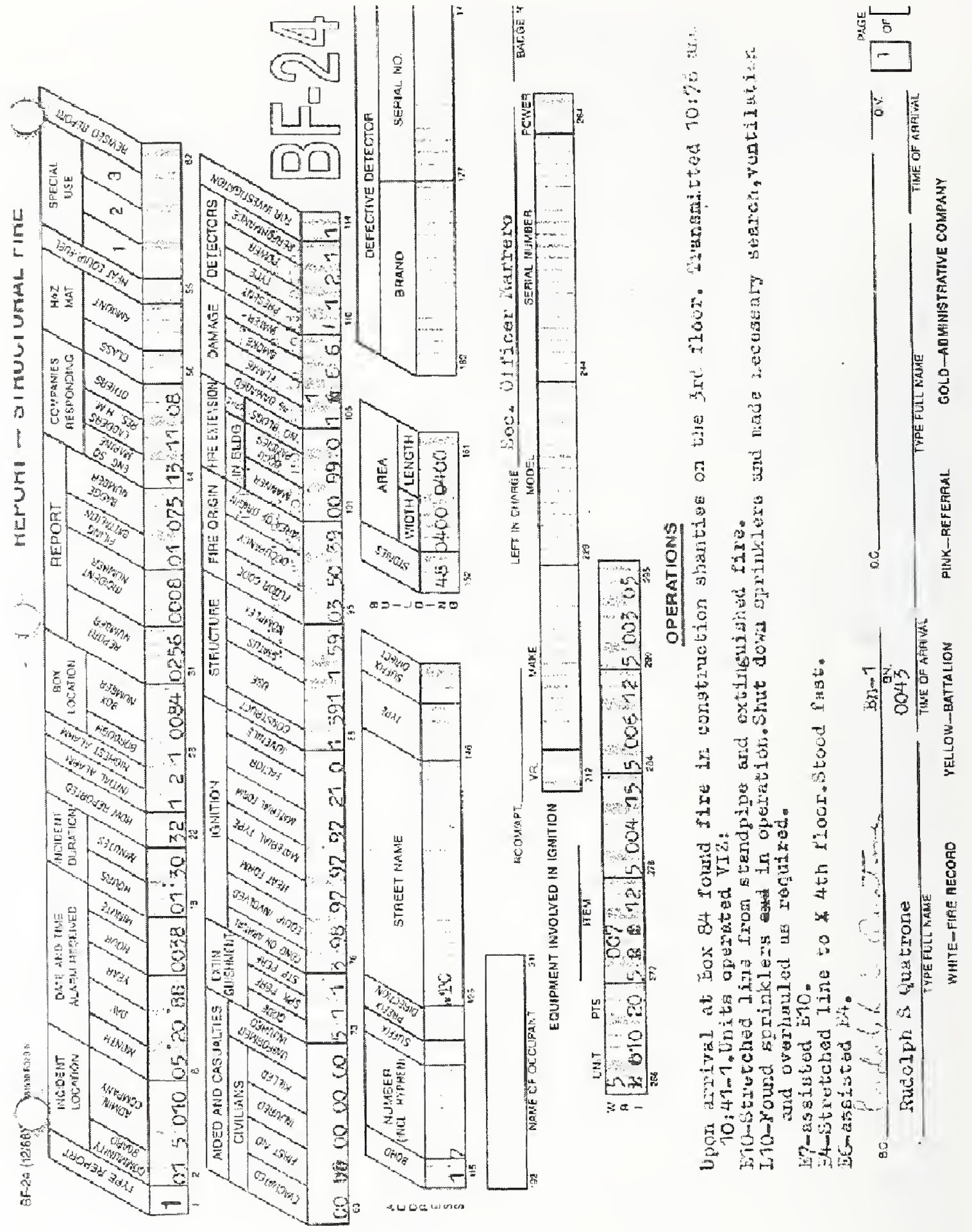


REPORT - ADDITIONAL DATA

STRUCTURAL FIRE, TRANSPORTATION FIRE, NON-STRUCTURAL FIRE OR EMERGENCY
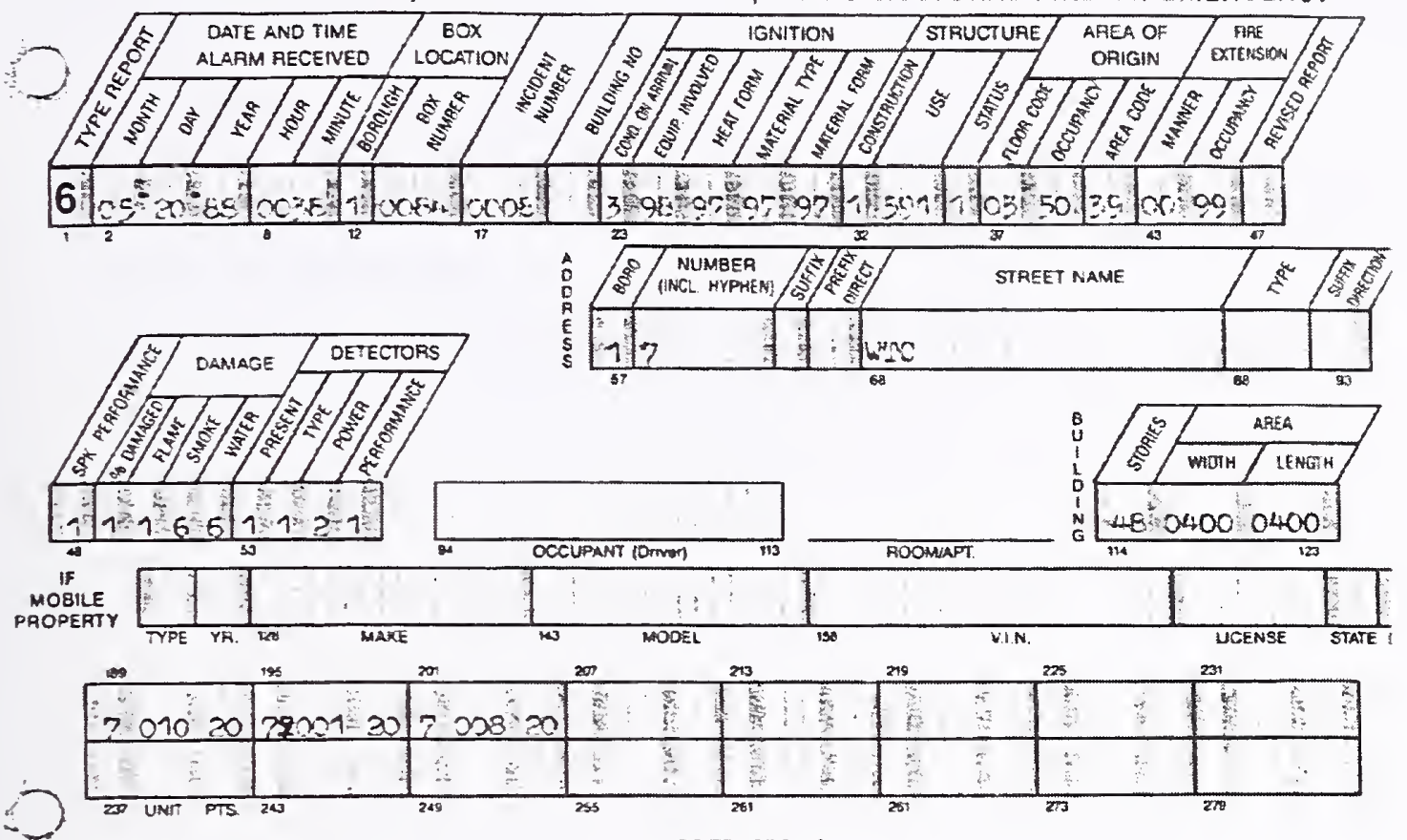

OPERATIONS

Oofrations as followe;

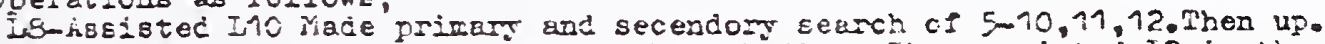
If liace search end exemination of the 4th floor. Iben asaisted is in the exeinetion c? Liper floom. Then up.

jatt 4 Supervised $11 C$ and 210 or fire floc:

Batt 2-SupeIvieed 54 azd 56 ard IT on floors above fire.

FCU: - SOP

MSU- SCP

Ez- Stood fast.

Bett 6-Stocd fest.

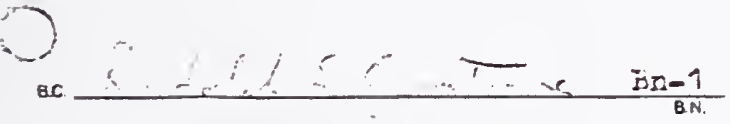

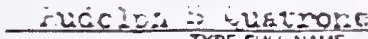

0

TYPE FULL NAME

oc

[]$^{\mathrm{Pa}}$ 
This page intentionally left blank. 


\section{Appendix I \\ Extracted Page from PACO Report on Sprinkler Program}

PACO 2002 Report: World Trade Center General Description of All Building Systems and the Capital Program. Extracted page.

Miscellaneous Life Safety Improvements and Sprinklerization Program 
12. Miscellaneous Life Safety Improvements and Sprinklerization Program

\subsection{Description of the Procram}

The inilial base building provided for Fre Standpipe (FSP) protection in the Towers and Plaza Buildings and no sprinkler system installation fexcept in the sub-grade Levels). In response to the enactment of Local Law 5 and other NYC Bulding Code Loca! Law enactments related to the fire protection of high rise cfice towers buit after 1973 the Port Authority voluntarily retrofited the WTC complex to comply with the new NYC Building Code Requirements supplemented by PA imposed life safely requirements unique to the WTC. These requirements provided tenants the option of achieving fire protection compliance by a compartmentalization of a sprinklerization option. The Scope of miscellaneous life safety and sprinkier system upgrades/improvements, other than certain fire alarm, concourse circulation and blast recovery improvements discussed elsewhere, included but was not limited to:

\subsection{Scope of Fire Life Safety Improvements}

Archilectural Modifications - On all foors:

- Upgraded core wall construction to provide for a 2 hour rated me separation from one side of a tower hoor to the opposite side (Where core wall were already 2 hour rated, such as in elevator shafts, upgraded where not previously required).

- Installed a double acting set of rated HM fire doors in the core corridors where the above 2 hour rated lire separation crossed the corridor (This enabled one half of a typical tower lloor to serve as a horizontal fire refuge for the other.) Doors included special magnetic hold open hardware and closures linked to smoke detectors in floor return air ducts.

- Restored all ceilings in corridors and lobbies affected by fire protection installations.

- Provided slab-to-stab thour fire rated walls to enable a maximum of 10,000 gross square foot compartmentalization on each side of the 2 hour rated fire separations.

Suinkler System Installation

- The entire WTC complex was fully sprinklered. The Sprinkler System was insialled in three basic phases. Phase 1: Sub-grade areas for the initial building construction; Phase 2: Sprinkler riser/main installation throughout 1 \& 2 WTC including the sprinklerization of corridors, storage rooms, lobbies and certain tenanUPA spaces in 1976 in complance with Local Law 5 to provide tenants with a compartmentation or sprinkler fire protection option; Phase 3: The full sprinklerization of the entire complex for all remaining places from 1983 to the 2001 (including 1993 blast recovery and ongoing up-gradesimprovements and replacements).

- The Tower sprink3er systems were led in the various zones from the gravily feed fire reserve tanks located on the $20^{\text {b }}, 41^{31}, 75^{\text {th }}$ and $110^{\text {th }}$ Floors through a $4^{\prime \prime}$ vertical sprinkler riser localed in the Janitor Closet on each foot of each builing in the WTC complex.

Reproduced with permission of The Port Authority of New York and New Jersey. 

